\title{
A Weather Pattern Climatology of the United States
}

W. R. Barchet

W. E. Davis

January 1984

Prepared for the U.S. Department of Energy under Contract DE-AC06-76RLO 1830

Pacific Northwest Laboratory Operated for the U.S. Department of Energy by Battelle Memorial Institute 


\title{
DISCLAIMER
}

This report was prepared as an account of work sponsored by an agency of the United States Government. Neither the United States Government nor any agency thereof, nor any of their employees, makes any warranty, express or implied, or assumes any legal liability or responsibility for the accuracy, completeness, or usefulness of any information, apparatus, product, or process disclosed, or represents that its use would not infringe privately owned rights. Reference herein to any specific commercial product, process, or service by trade name, trademark, manufacturer, or otherwise, does not necessarily constitute or imply its endorsement, recommendation, or favoring by the United States Government or any agency thereof. The views and opinions of authors expressed herein do not necessarily state or reflect those of the United States Government or any agency thereof.

\author{
PACIFIC NORTHWEST LABORATORY \\ operated by \\ BATTELLE \\ for the \\ UNITED STATES DEPARTMENT OF ENERGY \\ under Contract DE-AC06-76RLO 1830
}

\begin{tabular}{|c|c|}
\hline \multicolumn{2}{|c|}{ Printed in the United States of America } \\
\hline \multicolumn{2}{|c|}{ Available from } \\
\hline \multirow{2}{*}{\multicolumn{2}{|c|}{$\begin{array}{l}\text { National Technical Information Service } \\
\text { United States Department of Commerce }\end{array}$}} \\
\hline & \\
\hline \multicolumn{2}{|c|}{5285 Port Royal Road } \\
\hline \multicolumn{2}{|c|}{ Springfield, Virginia 22161} \\
\hline \\
\hline \multicolumn{2}{|c|}{ Microfiche A01 } \\
\hline \multicolumn{2}{|c|}{ Printed Copy } \\
\hline & Price \\
\hline Pages & Codes \\
\hline $001-025$ & $\mathrm{~A} 02$ \\
\hline $026-050$ & $\mathrm{~A} 03$ \\
\hline $051-075$ & A04 \\
\hline $076-100$ & A05 \\
\hline $101-125$ & A06 \\
\hline $126-150$ & $\mathrm{~A} 07$ \\
\hline $151-175$ & $\mathrm{~A} 0 \mathrm{~B}$ \\
\hline $176-200$ & A09 \\
\hline $201-225$ & A010 \\
\hline $226-250$ & A011 \\
\hline $251-275$ & $\mathrm{~A} 012$ \\
\hline $276-300$ & A013 \\
\hline
\end{tabular}




\section{A WEATHER PATTERN CL IMATOLOGY \\ OF THE UNITED STATES}

W. R. Barchet
W. E. Davis

January 1984

Prepared for

the U.S. Department of Energy

under Contract DE-AC06-76RLO 1830

Pacific Northwest Laboratory

Richland, Washington 99352 
: 


\section{ACKNOWLEDGEMENTS}

The time series of weather pattern type were produced by Dr. P. F. Lester and $\mathrm{Mr}$. M. Burton and their team of weather pattern analysts at the San Jose State University under contract to the Pacific Northwest Laboratory (PNL). Accurate classifications and the tabulation of numerical data from surface weather charts require persistent interest and enthusiasm as well as the ability to learn and to apply many concepts of synoptic meteorology. The following individuals possessed these qualities to the extent that this project was successful: G. Ballard, L. Barnett, J. Brown, B. Denny, K. Hines, D. Matson, S. May, M. Miller, F. Nelson, S. Nemeth, D. Regalia, J. Scheve, S. Siegel, R. Veronda, T. Wilkinson.

Substantial portions of this report were taken from or based on work reported by Lester and Burton in 1982. Their dedication, skill in training and in guiding the team of analysts and their valuable suggestions and contributions on weather pattern classification are gratefully acknowledged.

At the Pacific Northwest Laboratory, the data processing work by Neal Hara, the contributions by Dr. Harlan Foote to the graphical presentations, and the typing of this manuscript by Debbie Atkin are acknowledged.

The preparation and publication of this report has been funded by the U.S. Department of Energy under Contract DE-AC06-76RLO 1830. 
. 
This work is an extension of a previous wind energy study of the climatology of weather patterns for the midwest by Barchet (1982). In this study the geographic domain covered the 48 conterminous states of the United States. The daily synoptic weather pattern was classified into nine types for the 10-year period January 1, 1969 to December 31, 1978. Weather pattern types were defined relative to the classical polar front model of a mid-latitude cyclonic storm system and its associated air masses. Guidelines for classifying weather patterns on an operational basis were developed. These were applied to 3652 daily surface weather maps to produce a time series of weather pattern type at 120 grid points of a 160 point, $3^{\circ}$ latitude by $4^{\circ}$ longitude array over the United States.

Statistics on the frequency of occurrence, persistence and alternation of weather patterns were calculated for each grid point. Summary statistics for the entire grid and for six regions were also presented. Frequency of occurrence and persistence were found to depend on the size and speed of movement of the weather pattern. Large, slow moving air masses had higher frequency of occurrence and longer persistence than small (fronts) or rapidly moving (or changing) features (fronts, storm centers). Some types showed distinct regional preferences. The subtropical maritime high occurred mainly in the south central and southeast.

An indeterminate weather pattern type accounted for those weather patterns that did not fit the polar front model or were too disorganized to be classified. The intermountain thermal low of the desert southwest was one such feature that dominated both frequency of occurrence and persistence in this region.

Alternation from one weather pattern to another followed the polar front model of a moving cyclonic storm. The tendency for anticyclonic weather patterns to become disorganized as they weakened was seen in the high percentage of these patterns that changed to an indeterminate pattern as they aged. 
Regional differences in alternation were associated with regional differences in frequency of occurrence.

The weather pattern climatology presented here can serve as a benchmark for similar climatologies derived from short periods in other years. By applying the classification guidelines developed here to these other periods, the climatological representativeness of these periods can be evaluated. 


\section{CONTENTS}

ACKNOWLEDGEMENTS

i i

SUMMARY

INTRODUCTION

APPROACH .

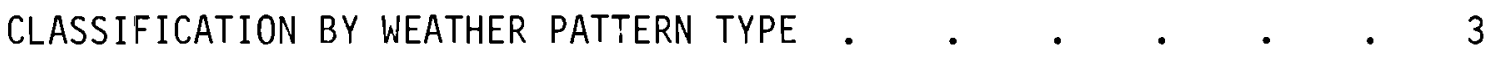

DATA BASE .

CLASSIFICATION PROCEDURE •

Map Analysis . . . . . . . . . . . . 6

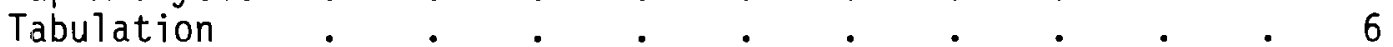

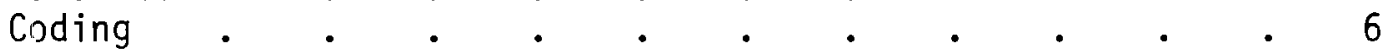

QUALITY CONTROL AND ASSURANCE .

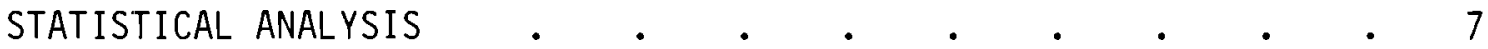

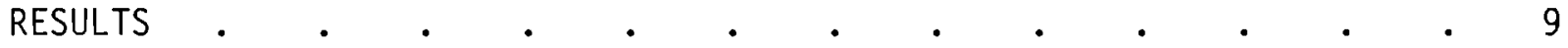

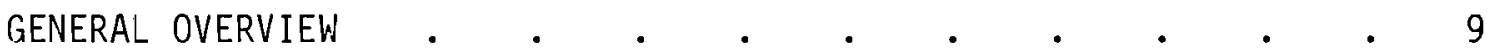

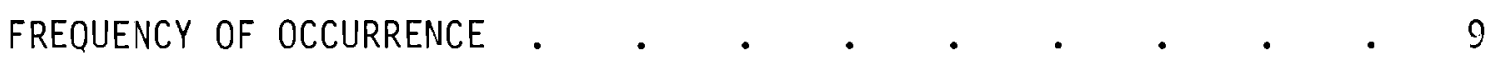

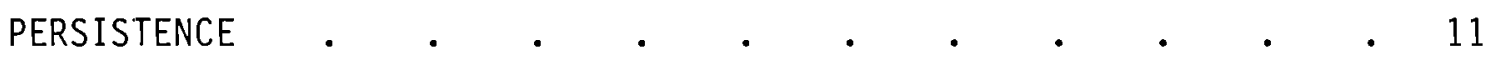

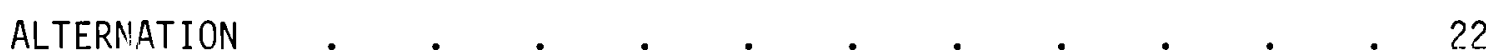

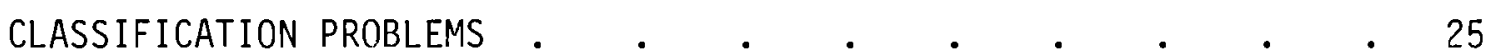

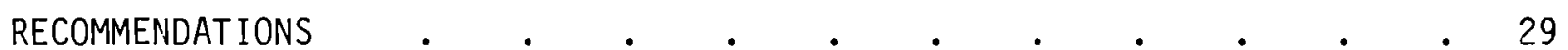

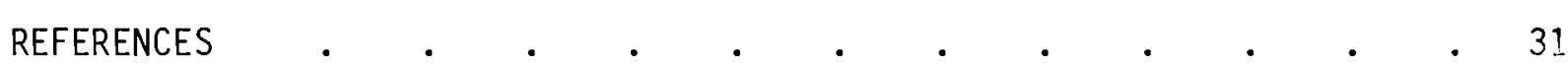

APPENDIX A - GUIDELINES FOR THE OPERATIONAL CLASSIFICATION

OF WEATHER PATTERNS $\quad \cdot \quad \cdot \quad \cdot \quad \cdot \quad \cdot \quad \cdot \quad \cdot \quad \cdot \quad \cdot \quad \cdot \quad \cdot \quad \cdot A-1$

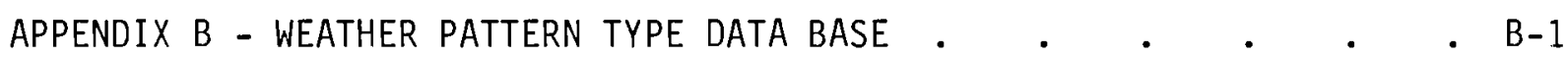

APPENDIX C - PERCENTAGE FREQUENCY OF WEATHER PATTERN OCCURRENCE • . - C-1

APPENDIX D - PERCENTAGE PROBABILITY OF RUN DURATION EXCEEDANCE • • • D-1

APPENDIX E - PERCENTAGE PROBABILITY OF WEATHER PATTERN

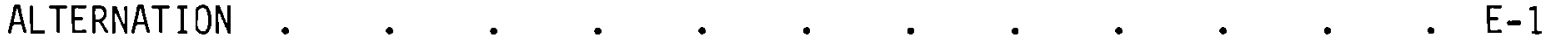




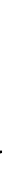




\section{LIST OF FIGURES}

1. 120 Array at $3^{\circ}$ Latitude by $4^{\circ}$ Longitude Spacing at Which Weather Pattern Type Time Series Were Produced for the Continental

U.S.A.

2. Schematic of Weather Pattern Types Associated With Surface Synoptic Weather Features . . . . . . . . 4

3. Annual and Seasonal Percentage Frequency of 0ccurrence and Variability of Weather Pattern Type 1: Warm Sector . . . 12

4. Annual and Seasonal Percentage Frequency of Occurrence and Variability of Weather Pattern Type 2: Ahead of Warm Front . . 13

5. Annual and Seasonal Percentage Frequency of Occurrence and Variability of Weather Pattern Type 3: Behind Cold Front . . 14

6. Annual and Seasonal Percentage Frequency of Occurrence and Variability of Weather Pattern Type 4: East of Continental High .

7. Annual and Seasonal Percentage Frequency of 0ccurrence and Variability of Weather Pattern Type 5: West of Continental High

8. Annual and Seasonal Percentage Frequency of Occurrence and Variability of Weather Pattern Type 6: Maritime High . . . 17

9. Annual and Seasonal Percentage Frequency of Occurrence and Variability of Weather Pattern Type 7: Stationary Front

10. Annual and Seasonal Percentage Frequency of Occurrence and Variability of Weather Pattern Type 8: Occluded Front and Regions West of Occluded Low. . . . . . .

11. Annual and Seasonal Percentage Frequency of 0ccurrence and Variability of Weather Pattern Type 9: Not Determinable . $\quad 20$

A.1. Type 1 (Warm Sector) . . . . . . . . . A-2

A.2. Type 2 (Ahead of Warm Front) . . . . . . . . A-4

A.3. Type 3 (Behind Cold Front) . . . . . . . . . A-5

A.4. Type 4 (East of a Continental High) and Type 5 (West of a
Continental High) . . . . . . . . . A-7

A.5. Type 8 (Occluded Front and Region West of Occluded Low) . A-9 


\section{$\underline{\text { LIST OF TABLES }}$}

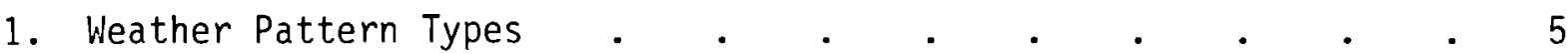

2. Percentage Coverage by Weather Pattern Type Averaged Over the 10-Year Period 1/1/69 to 12/31/78 . . . . . . . 10

3. Percentage Probability That a Weather Pattern Run Will

Exceed Current Duration Averaged Over the Entire Grid . . . 21

4. Probability That Current Run Terminates by Changing to New Weather Pattern Type $\quad \cdot \quad \cdot \quad \cdot \quad \cdot \quad \cdot \quad \cdot 23$

5. Number of Responses Specifying a Weather Pattern Type was

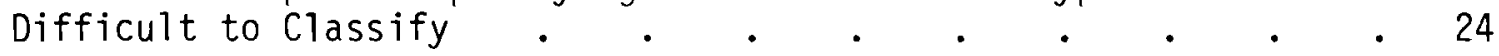

6. Synoptic Features Classified as Type 9: Indeterminate . . . 26

B.1. Logical Record Format for U.S.A. Weather Pattern Data Tape . - B-1

B.2. Block Format for U.S.A. Weather Pattern Data Tape . . . . B-2

B.3. Sample of the U.S. Weather Pattern Data Tape Content . . . B-2 


\section{$\underline{\text { INTRODUCTION }}$}

In an earlier report, Barchet (1982) described the use of weather pattern typing for characterizing the wind regime of the Great Plains. Weather patterns were based on the main features of fronts, and air masses associated with a Polar Front Model of a synoptic-scale cyclonic storm system. In a subsequent report, Barchet and Davis (1983) stratified the wind regime observed during a brief period of observation according to the weather pattern type and then used the long-term frequencies of weather pattern type occurrence to estimate the long-term mean wind speed. This report presents an extension of the Great Plains study of the frequency of weather pattern occurrence to the 48 conterminous states of the United States. The calculation and tabulation of two other related statistics were also completed. These were the persistence of a weather pattern type and the alternation of a weather pattern to a different type. These statistics can be used in estimating the frequency of frontal passages in any given region of the country. Such information could be valuable in an analysis of the performance of an array of wind turbines.

The work was done in two phases. Time series of weather pattern types were produced by a team of analysts at San Jose State University. Statistical analysis and graphical presentation of the time series data were done at the Pacific Northwest Laboratory (PNL). 
Classification of the weather patterns over the 48 conterminous United States followed guidelines established by Barchet (1982). Nine weather pattern types, based largely on isobar patterns, were defined. In the 1982 report, weather pattern time series were produced for a set of stations on the Great Plains covering the 10-year period January 1, 1955 to December 31, 1964. In contrast, for this report, weather pattern time series were produced at the regular array of 120 points shown in Figure 1 . The $3^{\circ}$ latitude by $4^{\circ}$ longitude grid covers the continental United States. The grid point in the upper left hand corner of Figure 1 is at $51^{\circ} \mathrm{N}, 126^{\circ} \mathrm{W}$. The 10 -year period covered the time from January 1, 1969 to December 31, 1978.

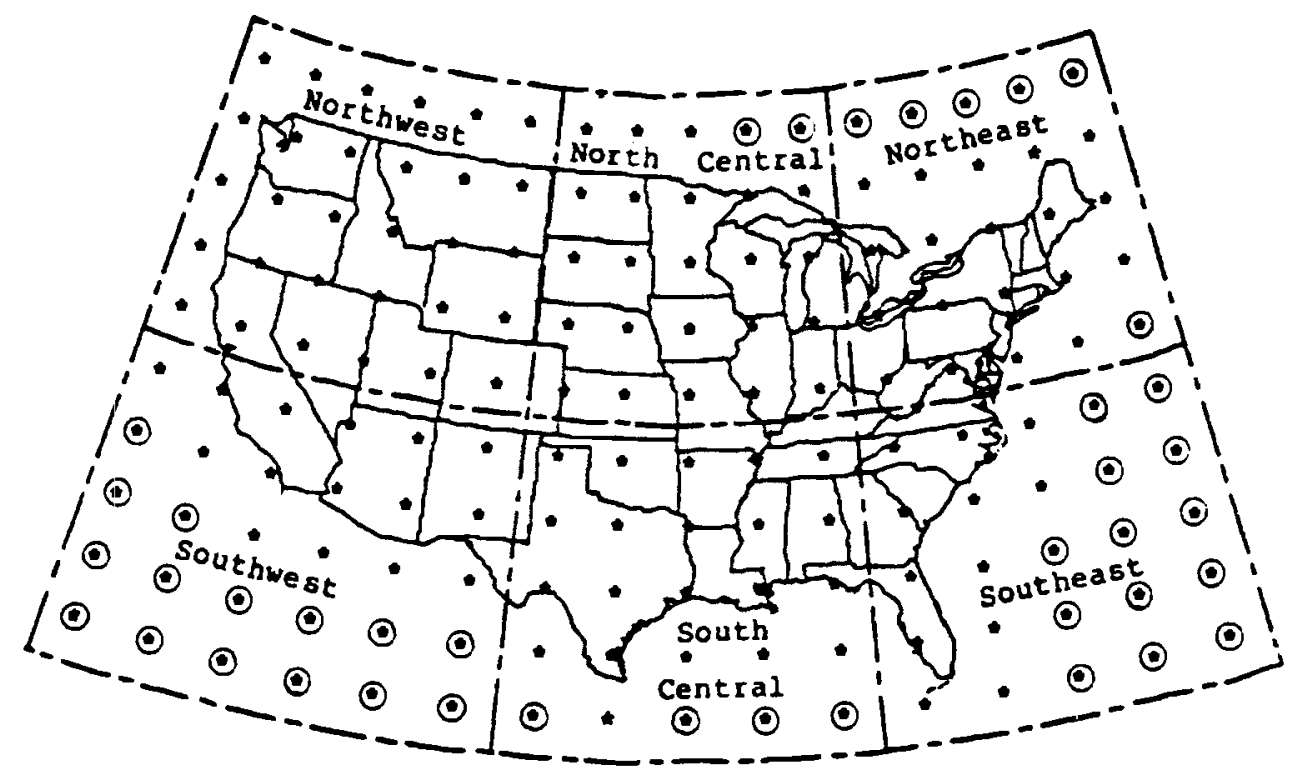

FIGURE 1. 120 Array at $3^{\circ}$ Latitude by $4^{\circ}$ Longitude Spacing at which Weather Pattern Type Time Series Were Produced for the Continental U.S.A. No weather pattern type time series were produced at the circled points.

\section{CLASSIFICATION BY WEATHER PATTERN TYPE}

The nine weather pattern types used in the 1982 study of the Great Plains are shown on a schematic of a large synoptic weather system in Figure 2 . A very brief description of the features of each type is given in Table 1. 


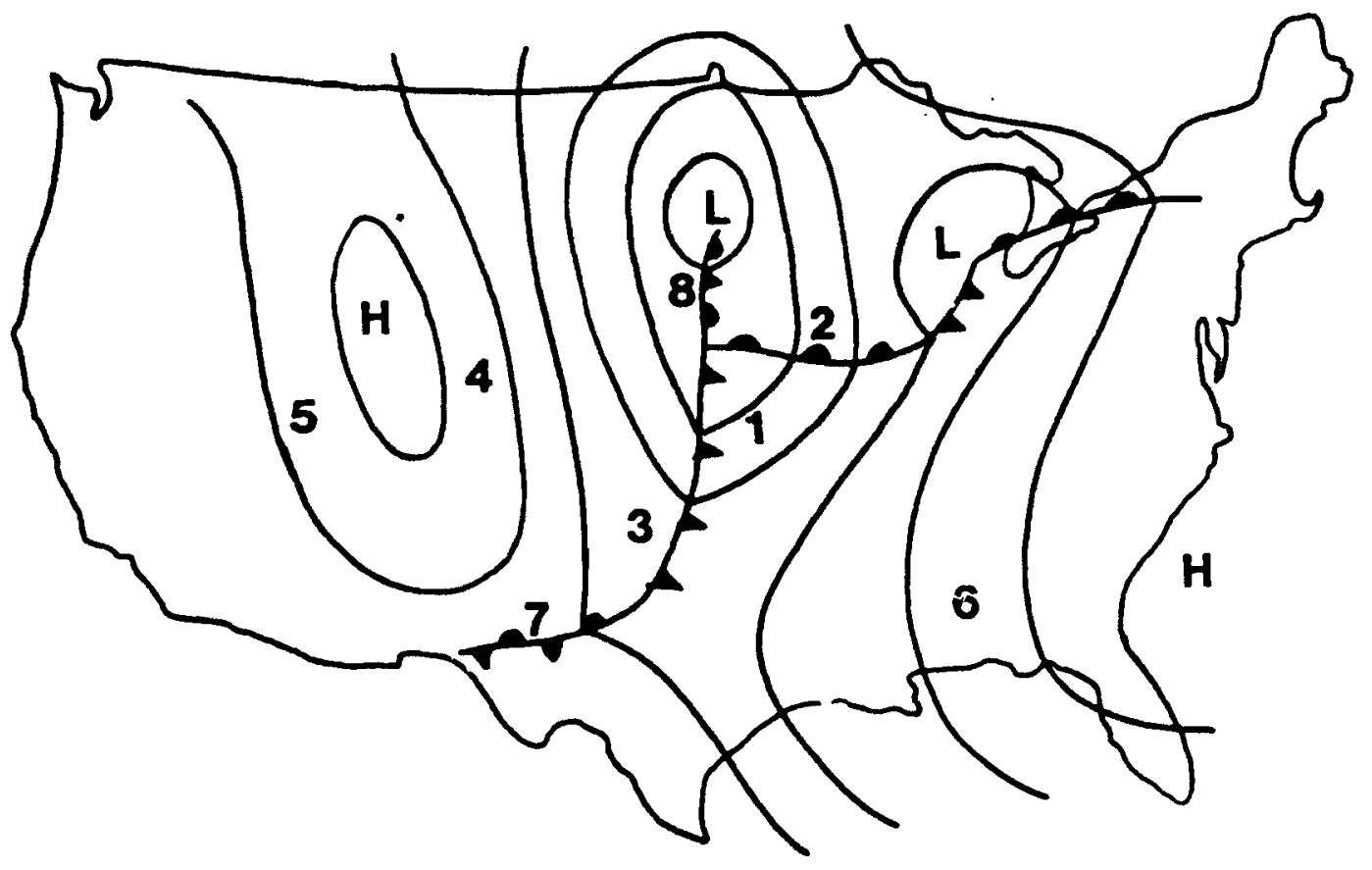

FIGURE 2. Schematic of Weather Pattern Types Associated With Surface Synoptic Weather Features

While these descriptions help portray the kind of weather associated with a particular weather pattern type, they do not provide sufficient detail or guidance to match unambiguously a particular weather pattern type to a given meteorological situation. Additional guidelines are included in Appendix $A$. These were developed to aid the operational classification of many weather situations.

\section{DATA BASE}

Weather pattern classification was done on 10 years (January 1, 1969 to December 31, 1978) of the 1200 GMT North American Surface Analysis prepared by the National Meteorological Center (NMC) of the National Weather Service. These maps are published weekly under the title Daily Weather Maps by the National Oceanic and Atmospheric Administration and are available from the Government Printing Office. The Daily Weather Map series was found superior to other formats for the NMC analyses, such as microfilm, because the $500 \mathrm{mb}$ 
TABLE 1. Weather Pattern Types

Type

1

Warm sector:

- Cyclonic curvature of isobars

- component of wind from south

- high humidity

- considerable clouds, fog possible

- some areas of precipitation

- pressure falling

2 Ahead of warm front:

- cyclonic curvature of isobars

- component of winds from east

- high humidity

- considerable clouds

- continuous precipitation

- pressure falling slowly

3 Behind cold front:

- cyclonic curvature of isobars

- component of wind from west

- intermediate humidity

- intermediate clouds

- shower-type precipitation

- pressure rising

4 East of continental high:

- anticyclonic curvature of isobars

- component of wind

- low humidity

- few clouds

- essentially no precipitation

- pressure steady or rising slowly

5 West of continental high:

- anticyclonic curvature of isobars

- component of wind from south
Type

Meteorological Situation

- low humidity

- fow clouds

- essentially no precipitation

- pressure steady or rising slowly

6

Maritime high:

- anticyclonic curvature of isobars

- component of wind from east

- intermediate-to-high humidity

- few-to-intermediate clouds

- some showers especially in afternoon

- pressure steady

Stationary front:

- indeterminate curvature of isobars

- light winds

- intermediate humidity

- variable cloudiness

- few areas of precipitation

- pressure steady

Occluded front and regions west of occluded low:

- cyclonic or indeterminate curvature of isobars

- component of winds from north

- high humidity

- considerable clouds

- considerable precipitation

- pressure rising slowly

9 Not determinable
No map, data missing 
analysis is presented on the same page and precipitation areas, that are important in the classification scheme, are clearly shown.

\section{CLASSIFICATION PROCEDURE}

The procedure by which time series of weather pattern type were produced for each of the 120 grid points shown in Figure 1 involved three steps. These are:

1. analyzing the daily surface weather maps for areas of homogeneous weather pattern type,

2. tabulating the weather pattern type analysis for the 120 grid points, and

3. coding of the tabulated data for computer processing and analysis.

Map Analysis

Each xerographic copy of the 3652 daily surface analyses was classified by dividing the map into areas which corresponded to the weather pattern types given in Table 1. The mechanics of this procedure simply required drawing the boundaries of each area of homogeneous type and numbering the center of the area with the pattern type.

\section{Tabulation}

Tabulations were accomplished by placing a transparent grid in registry with a classified map and subsequently tabulating the grid point values (types) on a tabulation form. The grid contains $160(16 \times 10)$ points, although data are only tabulated for 120 points. If a grid point fell on the boundary between two types, it was assigned the lower value.

$\underline{\text { Coding }}$

A commercial data entry organization keyed the tabulated data, one day (map) at a time directly into computer storage. The final data set was retrieved directly from the computer without further processing. See Appendix $B$ for a description of the format for the data base and data tape. 
QUALITY CONTROL AND ASSURANCE

A large team of analysts was involved in classifying the daily weather maps. To insure consistency between analysts, each member of the team underwent a 1-week training period. The first year of weather maps were classified by teams of two analysts with quality control provided by the project supervisors. For the remainder of the data, analyses were done individual1y and quality control was provided by a specially trained team of two analysts.

Quality control of the map classification focussed on the application of the guidelines presented earlier. Project supervisors spot checked classified maps and provided assistance with unusually difficult analyses.

Quality assurance in the tabulation of classified maps was provided by having a second person perform an independent tabulation which was cross checked against the origina1. A spot check of the tabulated data for consistency was done by a third analyst prior to coding the data.

Quality control on the keyed-in data was provided by comparing a listing of the data in computer storage against the tabulated data. A dump of the final data tape was spot checked against the original tapes as a final quality control.

\section{STATISTICAL ANALYSIS}

The principal statistic determined was the frequency of occurrence of each weather pattern type at each grid point. Four seasonal frequencies as well as an annual average frequency were calculated. Yearly frequencies were also calculated and used to produce the standard deviation of the yearly frequency of occurrence.

Two other related statistics were determined: the persistence of a weather pattern type at each grid point and the alternation of the weather pattern to a different type. Persistence was calculated from the number of consecutive days the weather pattern type did not change. Persistence is expressed as the probability that a run of a given weather pattern type will exceed its current duration. Alternation is expressed as the probability a run of one weather pattern type will be terminated by another type. 

$\underline{\text { RESULTS }}$

\section{GENERAL OVERVIEW}

The distribution of weather pattern types over the grid shown in Figure 1 for the period January 1, 1969 to December 31, 1978 is summarized in Table 2. The northwest region shows the highest count because it encloses the largest number of grid points at which weather pattern time series were produced. Table 2 highlights regional differences in the occurrence of various weather pattern types.

The western third of the grid (northwest and southwest) is dominated (46.5\%) by Type 9 weather patterns which do not fit those associated with the classical polar front model of a synoptic-scale cyclonic storm system. No area of the country was totally free of indeterminate weather patterns.

Traveling fronts, storm systems and air masses produce a more nearly uniform distribution of weather patterns over the northeast. Slow moving, large high pressure systems dominate. The percentage coverage of frontal and storm weather pattern types is rather uniform.

The south central and southeastern portions of the region are dominated by anticyclonic weather patterns (Types 4,5 and 6 ). The Florida peninsula, that is nearly half of the southeast, is usually under the maritime high pressure (Type 6) weather pattern. Continental highs frequently approach the southeast (Type 4) but seldom seem to move out to sea over the southeast coast. As a result Type 5 occurs less than $1 / 3$ as often as Type 4 in this region.

A few instances of missing map arose in the 10-year period. In most of these cases the Daily Weather Map cut off the very southern part of the grid so that no classification could be made.

\section{FREQUENCY OF OCCURRENCE}

The frequency of weather pattern occurrence was determined by summing the number of occurrences of a weather pattern type at a grid point and dividing by the total number of samples. Annual and seasonal time periods were analyzed. 
TABLE 2. Percentage Coverage by Weather Pattern Type Averaged Over the 10-Year Period $1 / 1 / 69$ to $12 / 31 / 78$

\begin{tabular}{|c|c|c|c|c|c|c|c|c|c|c|c|}
\hline & & & & & & ther $P_{i}$ & tern Ty & & & & \\
\hline Region & Count & 1 & 2 & 3 & 4 & 5 & 6 & 7 & 8 & 9 & 10 \\
\hline Northwest & 109560 & 1.9 & 6.2 & 2.5 & 21.9 & 19.3 & 0.0 & 10.6 & 4.6 & 32.9 & 0.0 \\
\hline North Central & 69388 & 4.1 & 8.7 & 3.5 & 26.0 & 26.7 & 2.5 & 11.5 & 7.5 & 9.6 & 0.0 \\
\hline Northeast & 83996 & 4.5 & 10.8 & 3.5 & 26.9 & 16.5 & 7.8 & 7.5 & 13.2 & 9.4 & 0.0 \\
\hline Southwest & 54780 & 0.8 & 0.6 & 1.3 & 7.3 & 11.1 & 0.2 & 4.2 & 0.8 & 73.6 & 0.0 \\
\hline South Central & 62084 & 2.0 & 1.3 & 1.6 & 14.0 & 16.9 & 35.9 & 10.7 & 0.6 & 17.1 & 0.0 \\
\hline Southeast & 58432 & 2.4 & 1.6 & 2.1 & 17.2 & 5.3 & 49.5 & 10.2 & 1.0 & 10.7 & 0.0 \\
\hline Total Grid & 438240 & 2.7 & 5.5 & 2.5 & 19.9 & 16.7 & 13.6 & 9.3 & 5.2 & 24.6 & 0.0 \\
\hline WPT Count & & 11802 & 23958 & 11031 & 87348 & 73147 & 59560 & 40842 & 22786 & 107755 & 11 \\
\hline
\end{tabular}


Seasonal periods are defined as:

$$
\begin{aligned}
& \text { Winter - December, January, February } \\
& \text { Spring - March, Apri1, May } \\
& \text { Summer - June, Ju1y, August } \\
& \text { Autumn - September, October November. }
\end{aligned}
$$

Appendix $C$ gives the percentage frequency of occurrence of the nine weather patterns at each grid point. Values are given for the annual and four seasonal averages as well as for the variability of the yearly frequency of occurrence. Variability is defined as the standard deviation of the 10 yearly mean frequency of occurrence divided by the annual or 10-year mean value. Appendix $C$ gives the frequency of weather pattern type at each grid point for each year the period 1969 through 1978.

These results are graphically summarized in Figures 3 to 11 , one for each weather pattern type. Contour intervals on these figures begin at $0.5 \%$, step to 1.0 and $2.0 \%$, and then are incremented by 2 percentage points to the maximum value contoured. The variability is expressed as a percentage of the annual mean frequency.

\section{PERSISTENCE}

Persistence refers to the number of consecutive days the same weather pattern type is found at a grid point. It is expressed as the percentage probability that the length of a run will exceed the current duration. Appendix $D$ gives the persistence by weather pattern type summarized by region. Probabilities of run durations from 1 to 7 days are shown for each grid point. The total number of runs is also given.

Summary statistics on run duration are presented in Table 3 for the entire grid. The probability of run duration exceeding a given duration decreases rapidly as run duration goes beyond 3 days. As is apparent from this table, weather patterns associated with large, slow moving features such as the continental highs (Types 4 and 5 ) and the nearly stationary maritime high (Type 6) tend to have longer runs than frontal features (Types 2 and 3 ) or cyclonic storm features (Types 1 and 8 ). The stationary front (Type 7) 

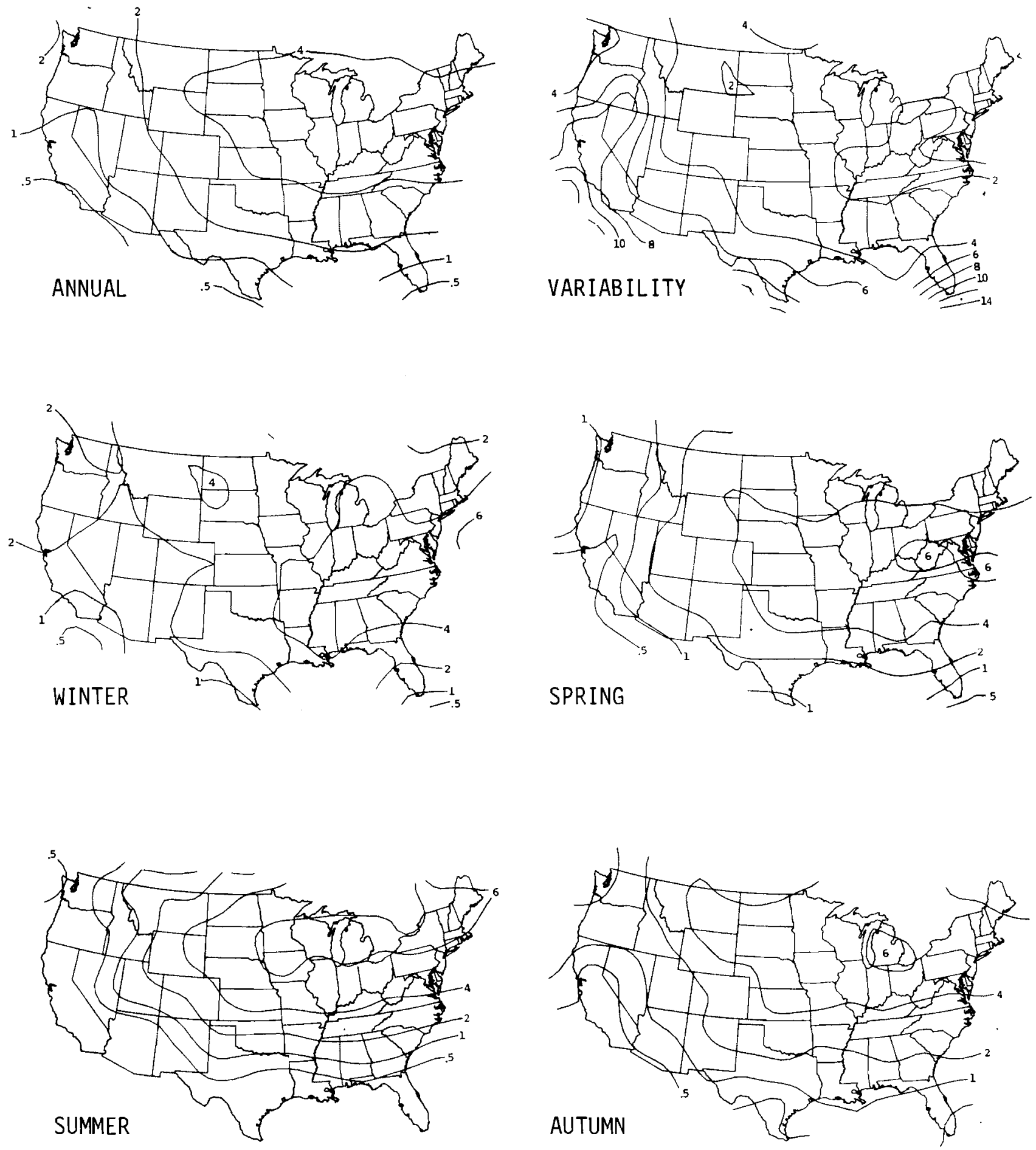

FIGURE 3. Annual and Seasonal Percentage Frequency of Occurrence and Variability of Weather Pattern Type 1: Warm Sector 

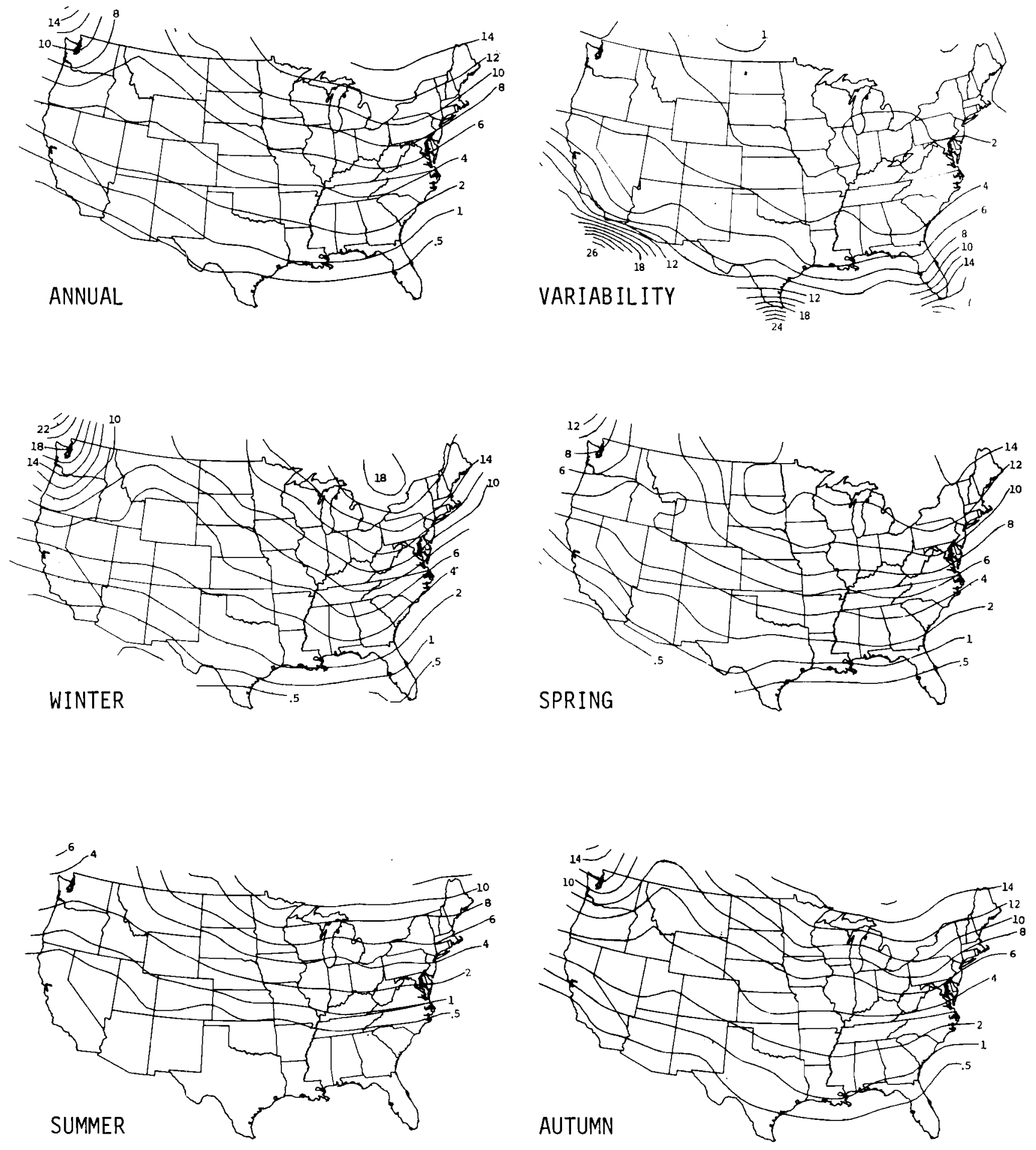

FIGURE 4. Annual and Seasonal Percentage Frequency of Occurrence and Variability of Weather Pattern Type 2: Ahead of Warm Front 

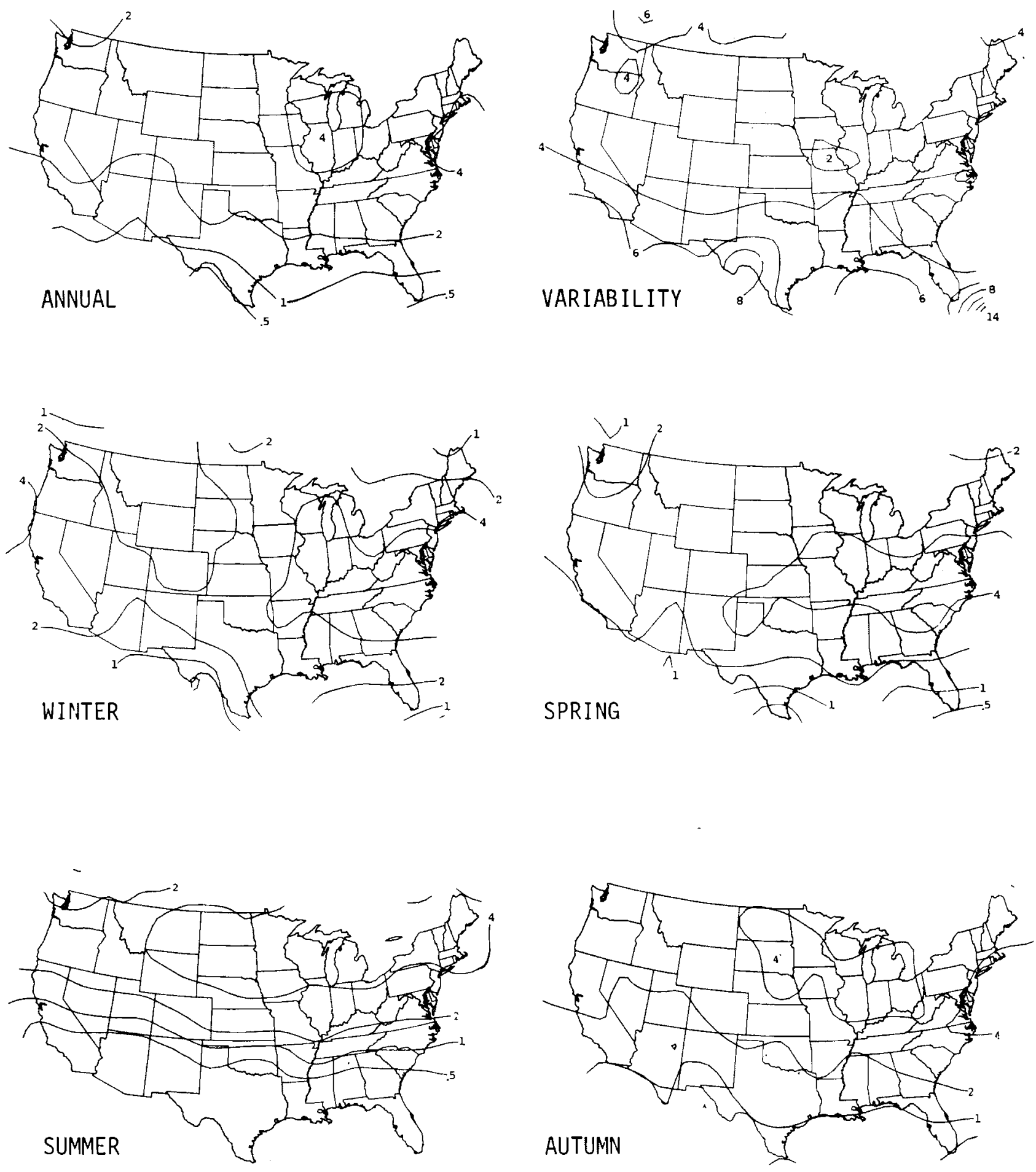

FIGURE 5. Annual and Seasonal Percentage Frequency of Occurrence and Variability of Weather Pattern Type 3: Behind Cold Front 

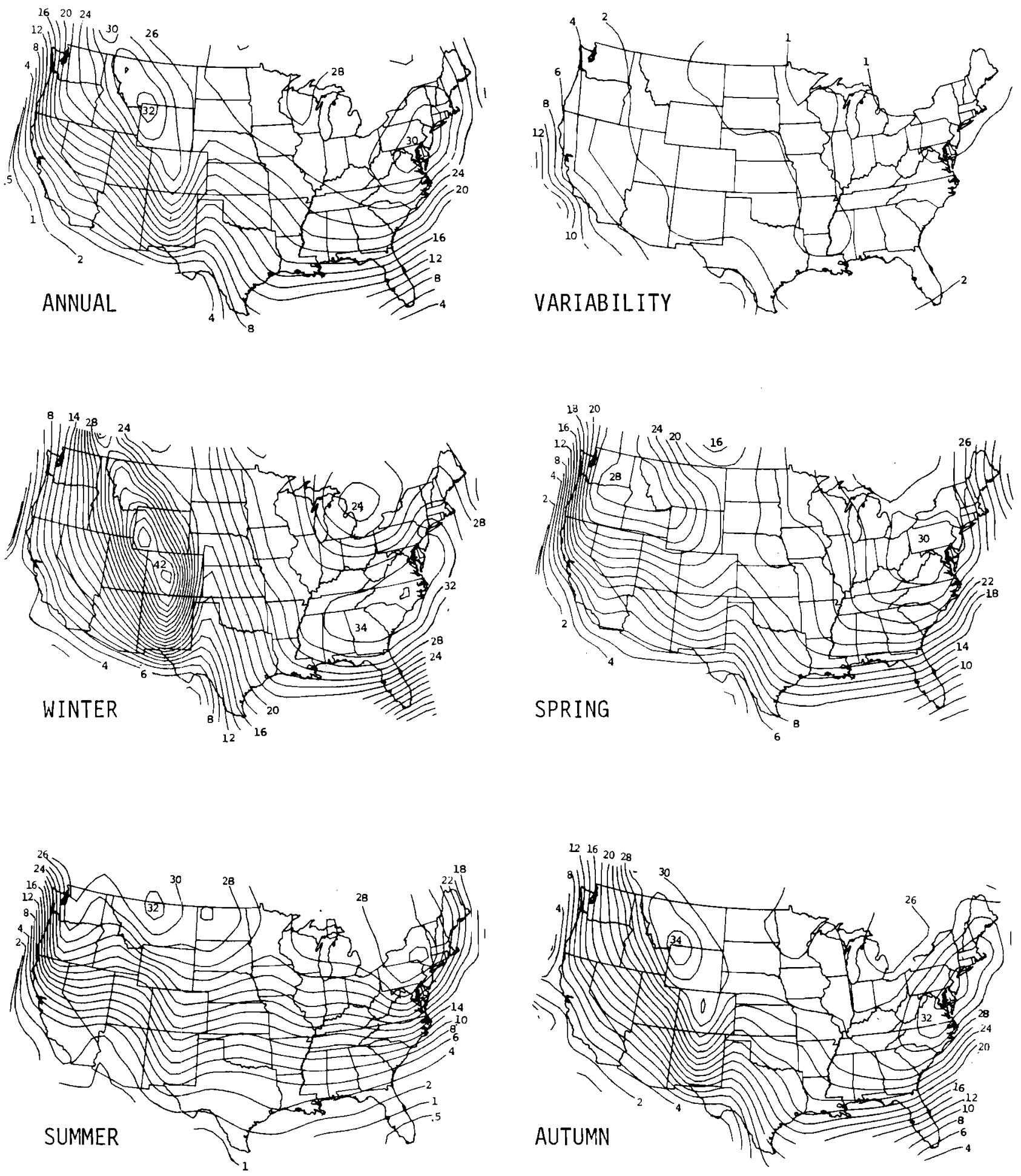

FIGURE 6. Annual and Seasonal Percentage Frequency of Occurrence and Variability of Weather Pattern Type 4: East of Continental High 

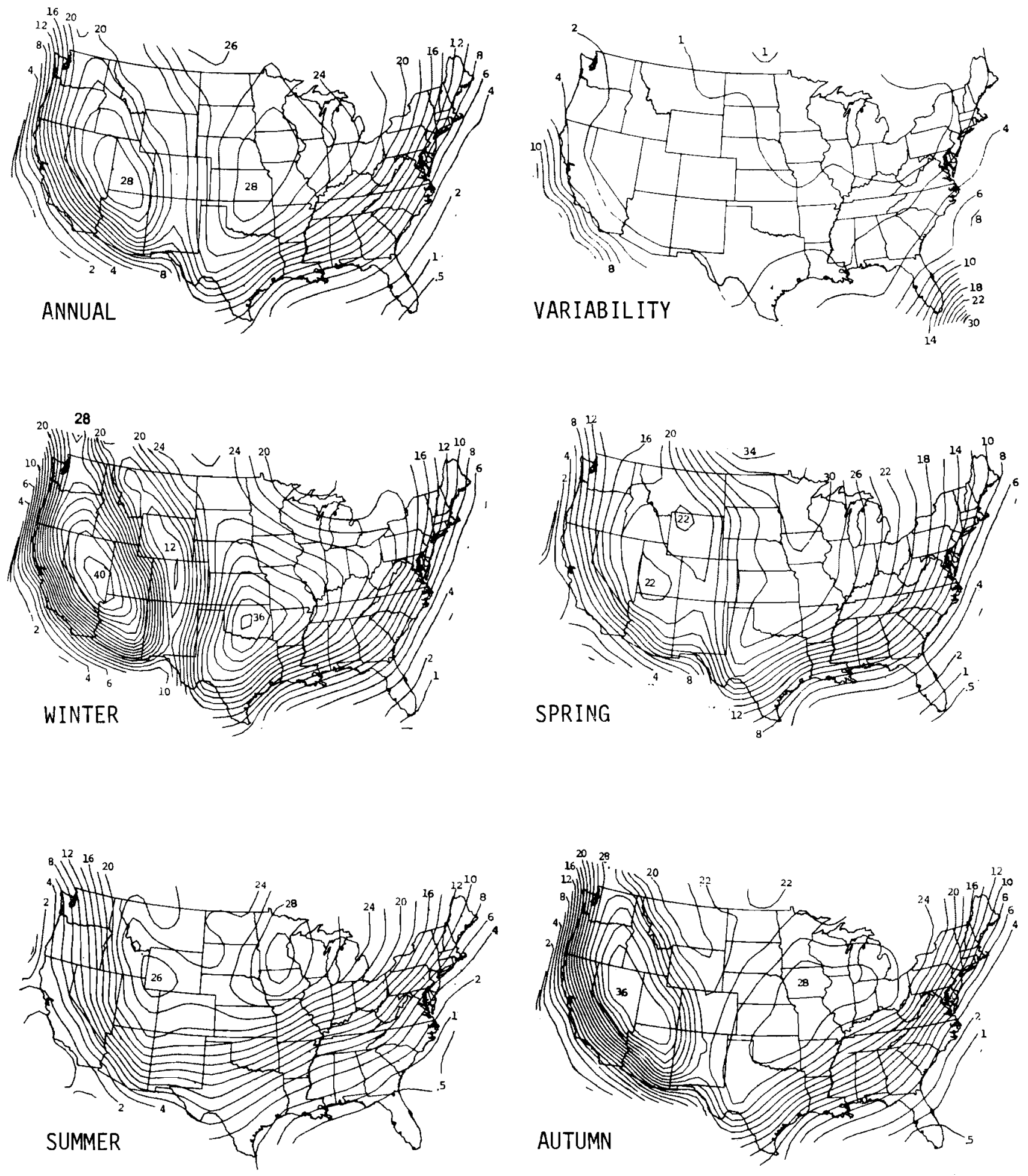

FIGURE 7. Annual and Seasonal Percentage Frequency of Occurrence and Variability of Weather Pattern Type 5: West of Continental High 

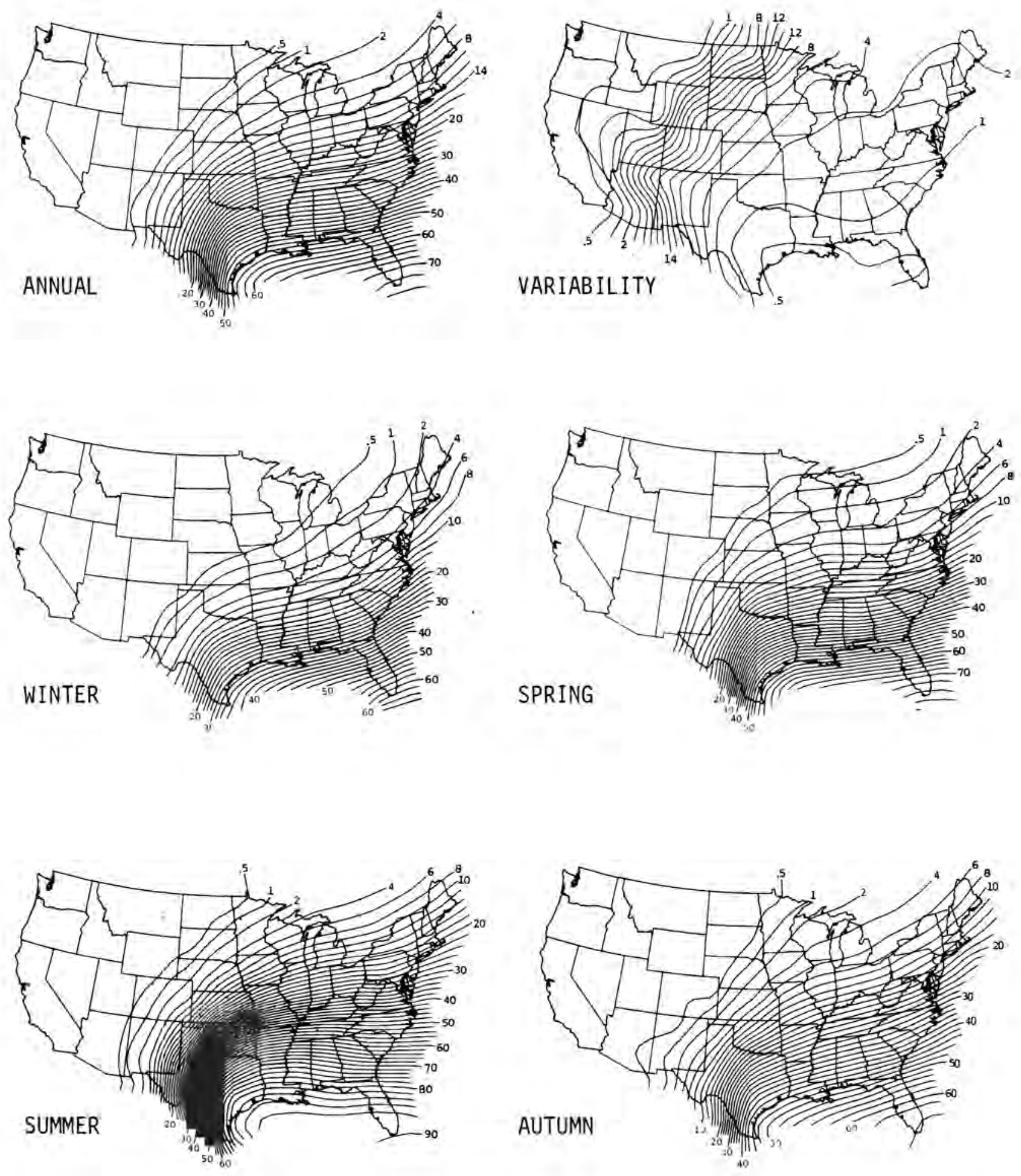

FIGURE 8. Annual and Seasonal Percentage Frequency of Occurrence and Variability of Weather Pattern Type 6: Maritime High 

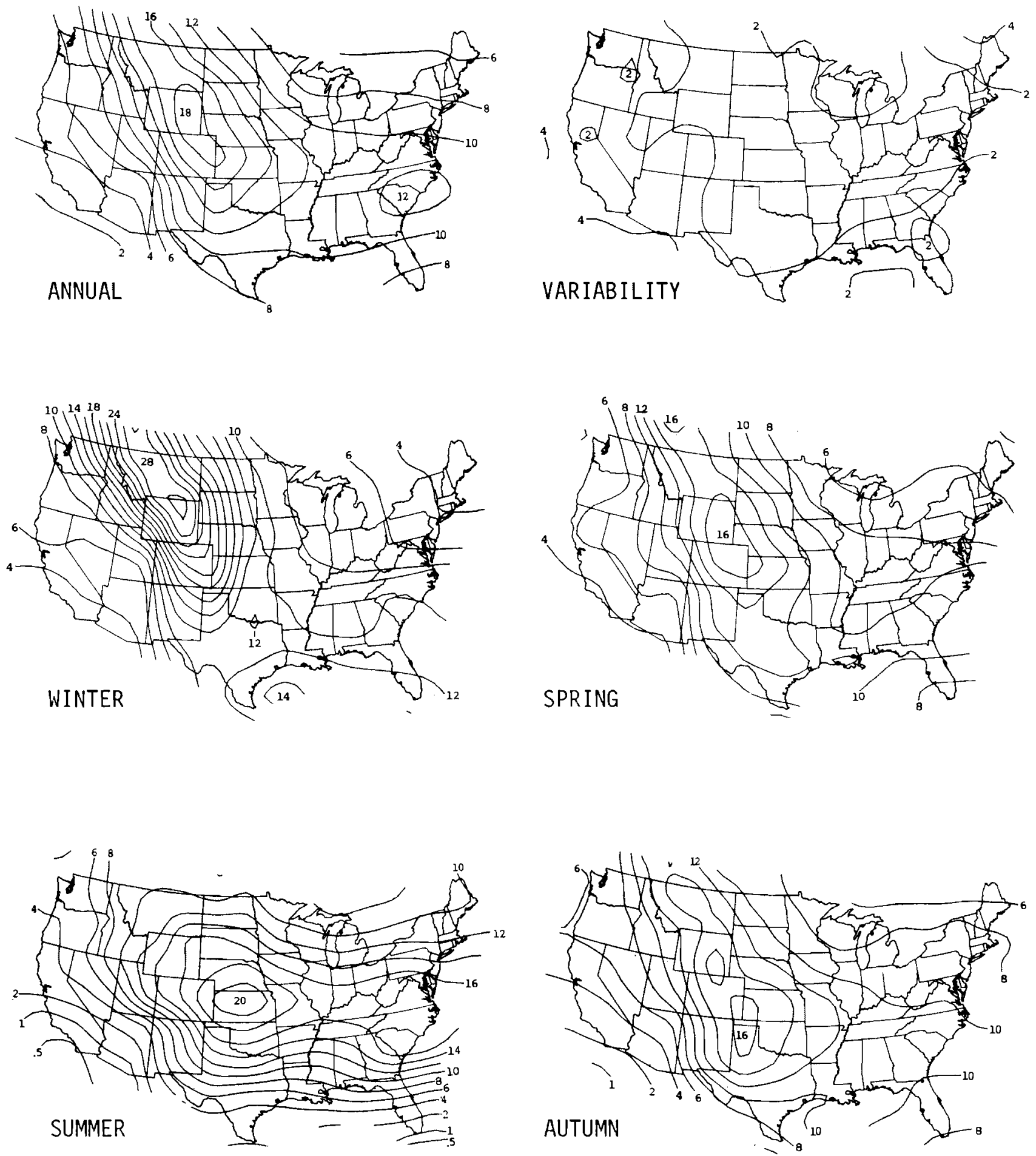

FIGURE 9. Annual and Seasonal Percentage Frequency of Occurrence and Variability of Weather Pattern Type 7: Stationary Front 

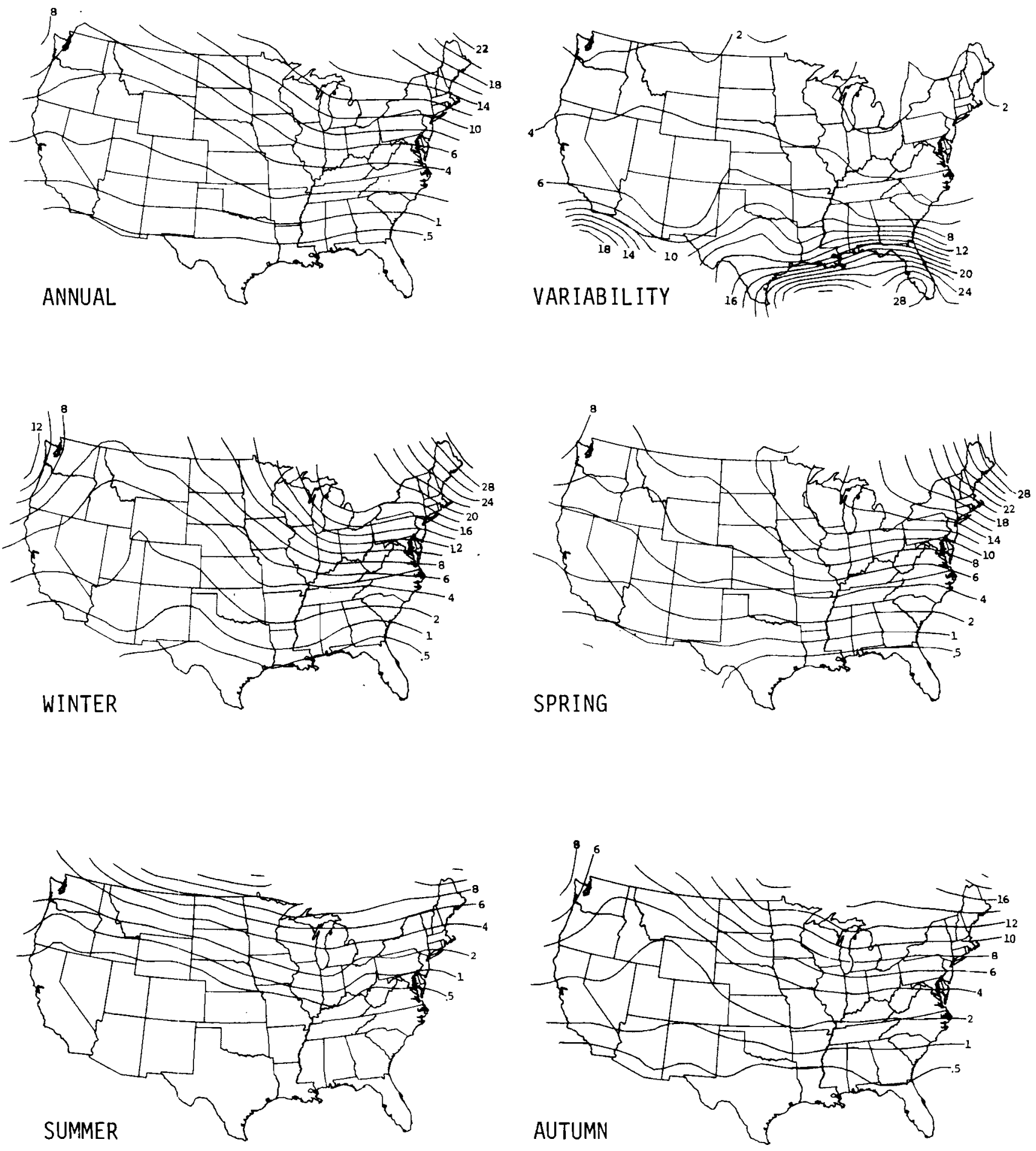

FIGURE 10. Annual and Seasonal Percentage Frequency of Occurrence and Variability of Weather Pattern Type 8: 0ccluded Front and Regions West of Occluded Low 

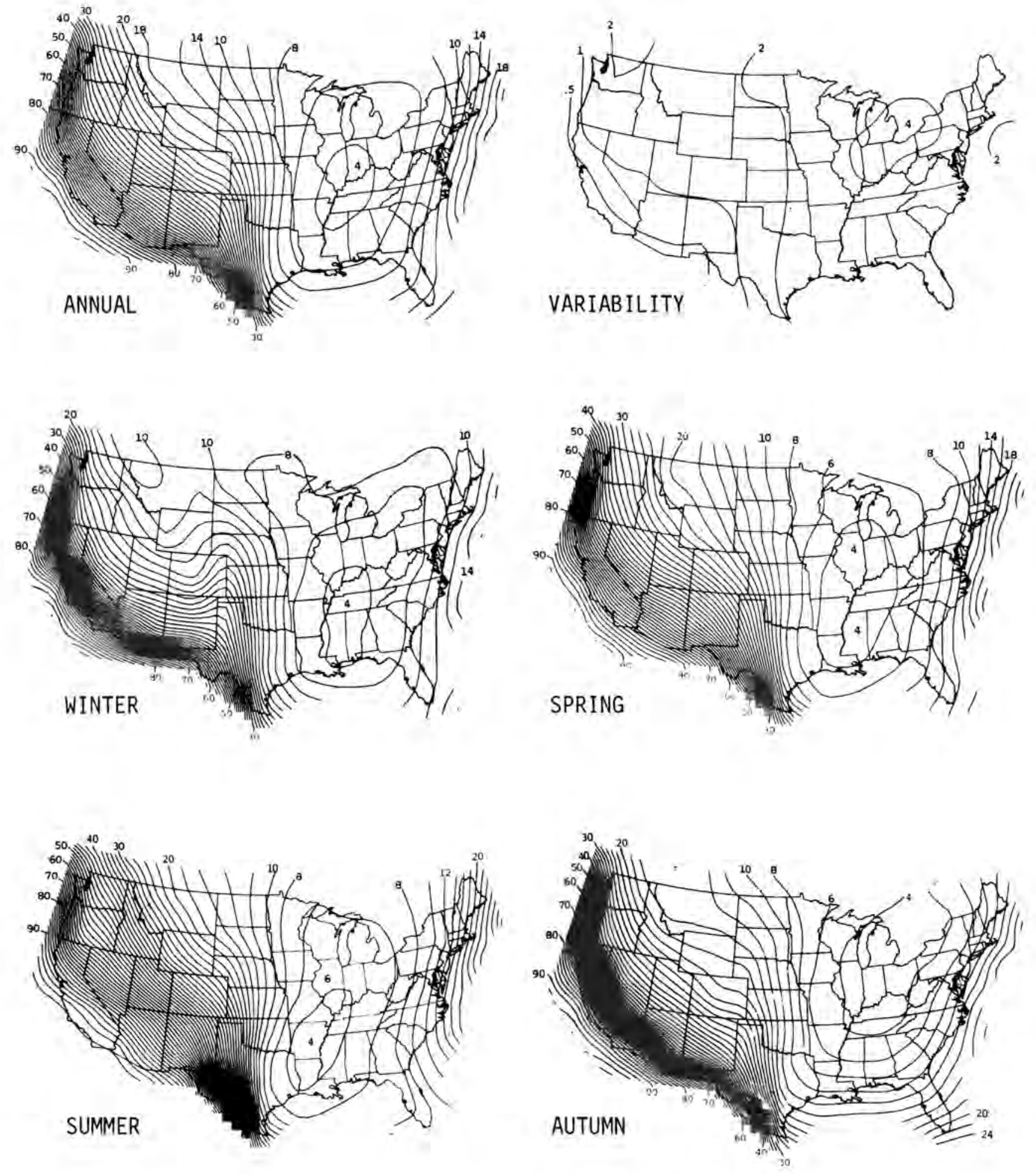

FIGURE 11. Annual and Seasonal Percentage Frequency of Occurrence and Variability of Weather Pattern Type 9: Not Determinable 
TABLE 3. Percentage Probability That a Weather Pattern Run Will Exceed Current Duration Averaged Over the Entire Grid

\begin{tabular}{|c|c|c|c|c|c|c|c|c|c|}
\hline \multirow[b]{2}{*}{ Days } & \multicolumn{9}{|c|}{$\begin{array}{cc}\text { Current Run Weather Pattern Type } \\
4\end{array}$} \\
\hline & 1 & 2 & 3 & 4 & 5 & 6 & 7 & 8 & 9 \\
\hline 1 & 6.2 & 12.1 & 3.1 & 35.2 & 33.3 & 51.0 & 22.1 & 23.8 & 37.0 \\
\hline 2 & 0.5 & 1.8 & 0.1 & 12.4 & 11.9 & 31.8 & 5.7 & 6.1 & 19.1 \\
\hline 3 & 0.1 & 0.4 & 0.0 & 4.9 & 4.8 & 21.3 & 1.6 & 2.3 & 11.9 \\
\hline 4 & 0.0 & 0.1 & 0.0 & 2.0 & 2.0 & 15.4 & 0.6 & 1.0 & 8.4 \\
\hline 5 & 0.0 & 0.0 & 0.0 & 0.9 & 0.9 & 11.7 & 0.2 & 0.5 & 6.4 \\
\hline 6 & 0.0 & 0.0 & 0.0 & 0.4 & 0.5 & 9.1 & 0.1 & 0.2 & 5.0 \\
\hline 7 & 0.0 & 0.0 & 0.0 & 0.2 & 0.3 & 7.4 & 0.1 & 0.1 & 4.2 \\
\hline $\begin{array}{l}\text { Tota } 1= \\
\text { Runs }\end{array}$ & 11048 & 20916 & 10695 & 55834 & 47503 & 20048 & 31273 & 16979 & 44430 \\
\hline
\end{tabular}


longer duration runs than the other frontal features. Type 9, the indeterminate classification, frequently has long runs, especially in the southwest.

Regional differences in persistence are summarized in tables in Appendix $D$. In general, persistence is correlated with the frequency of occurrence. In regions where the occurrence of a weather pattern is rare, its persistence is also low. The converse also applies. For example, Type 6, maritime high, shows large regional differences in persistence that match its regional frequency of occurrence. The dominance of Type 9 , indeterminate, in the southwest is a result of the warm-season thermal low over the intermountain basin. In the southeast, that is Florida and the western Atlantic, Type 6, the maritime high (Bermuda high), dominates the persistence as well as frequency of occurrence. The stationary character of the thermal $10 \mathrm{w}$ and the maritime high contribute to their long persistence.

\section{ALTERNATION}

Runs of a weather pattern type are terminated when the weather pattern changes. Statistics on the alternation of weather patterns have been calculated for all points on the grid and are summarized by region in Appendix $E$. The values given in Table 4 express the probability that the current run of a weather pattern will end by changing to the new weather pattern.

The probabilities of transition from one weather pattern to another coincide with what is expected from the movement of the synoptic system as diagrammed in Figure 2. Passage of a cold front replaces the warm sector (Type 1) with the east side of the continental high (Type 4). In turn, as that high moves, Type 4 is replaced with Type 5 . The western side of the high leads to an area of indistinct pressure gradients and thus to many Type 9 classifications. The maritime or subtropical high over the southern coastal areas is often bordered by a stationary front (Type 7). When this front begins to move, it usually moves eastward allowing the continental high (Type 4) to move in. The continental high, with large probability, replaces the occluded low type as the storm systems move along the storm track. Indeterminate weather patterns of Type 9 rather uniformly either follow or evolve into Types $4,5,6$ and 7 . These four weather patterns are the most 
TABLE 4. Probability That Current Run Terminates by Changing to New Weather Pattern Type

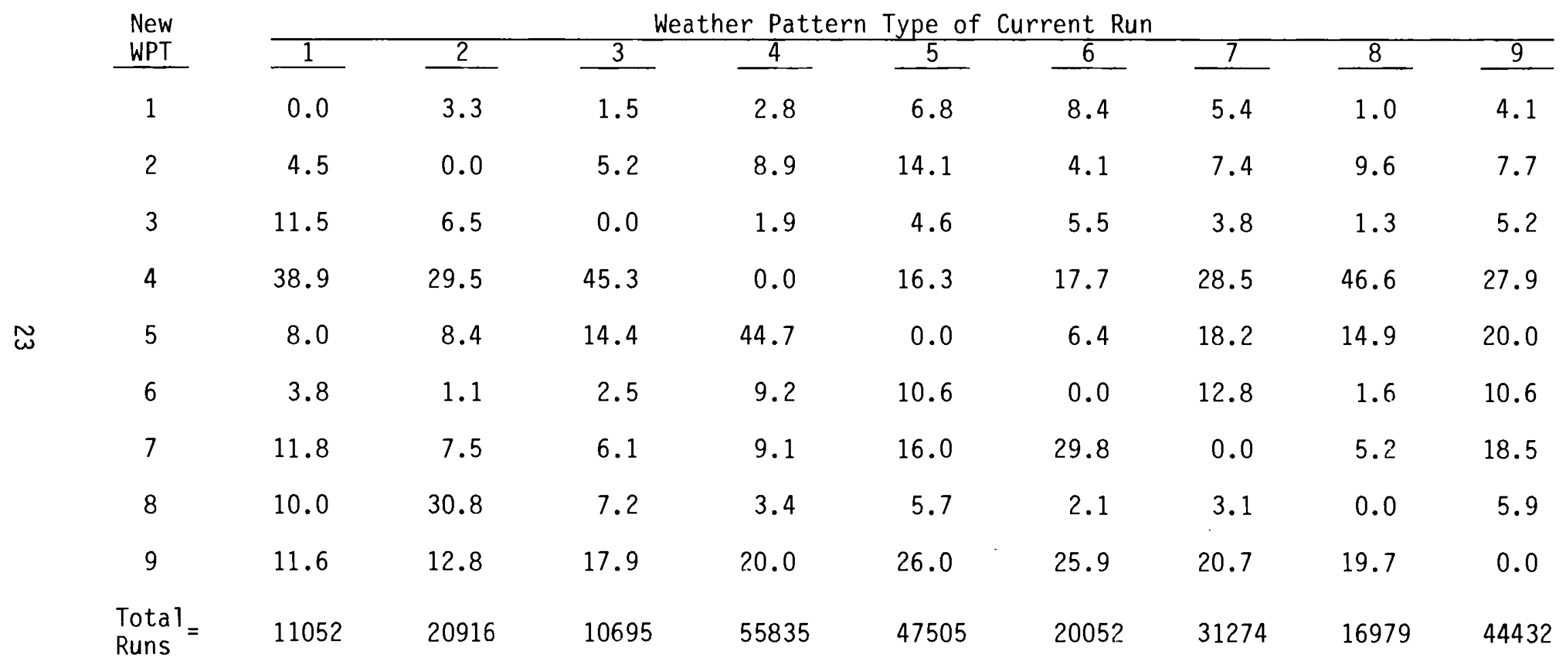


likely to be associated with weak, unorganized pressure fields that do not fit the Polar Front Mode1; especially as these air masses leave their source regions, weaken and undergo transition.

Regional differences in the alternation frequency follow the pattern given in Table 5. However, where the regional frequency of occurrence differs substantially from the total grid average, some shifts in the alternation pattern occur. For example, in the Northwest, Type 6 is virtually absent but Type 9 is common, hence alternation to Type 9 is more frequent for a11 other types while alternation to Type 6 never has a chance to occur.

TABLE 5. Number of Responses Specifying a Weather Pattern Type was Difficult to Classify

\begin{tabular}{|c|c|c|c|c|c|c|c|c|c|c|}
\hline \multirow[b]{2}{*}{ Area } & \multicolumn{9}{|c|}{ Type } & \multirow[b]{2}{*}{ Total } \\
\hline & $\underline{1}$ & $\underline{2}$ & $\underline{3}$ & 4 & 5 & 6 & $\underline{7}$ & 8 & 9 & \\
\hline $\begin{array}{l}\text { Pacific } \\
\text { Northwest }\end{array}$ & 1 & 2 & & 2 & 1 & & & 3 & 3 & 12 \\
\hline West Coast & 1 & 1 & 2 & 4 & 2 & & 2 & 2 & 3 & 17 \\
\hline Southwest & 1 & & & & & & & & 4 & 5 \\
\hline Rockies & 1 & 1 & 1 & & & & 3 & 1 & & 7 \\
\hline $\begin{array}{l}\text { Northeast } \\
\text { Coast }\end{array}$ & & & & 4 & 3 & 1 & & 3 & 1 & 12 \\
\hline $\begin{array}{l}\text { Southeast } \\
\text { Coast }\end{array}$ & & & & 4 & 4 & 3 & 1 & & & 12 \\
\hline $\begin{array}{l}\text { Gulf } \\
\text { States }\end{array}$ & & & & 1 & 2 & 3 & 1 & & & 7 \\
\hline Caribbean & & & & & & & 1 & & & 1 \\
\hline Atlantic & & & & 1 & & 1 & & & & 2 \\
\hline Plains & 1 & 1 & 1 & & & 2 & & 1 & & 6 \\
\hline Tota 1 & 5 & 5 & 4 & 16 & 12 & 10 & 8 & 10 & 11 & \\
\hline
\end{tabular}




\section{CLASSIFICATION PROBLEMS}

The scheme for classifying weather pattern types is based on an idealized model of distinct weather patterns associated with very well defined largescale weather systems. In reality, however, weather systems are seldom so distinctly structured; nor are sharp discontinuities in the features that characterize various weather pattern types always present. Three problems generally confronted the analysts:

1. The differentiation between certain weather pattern types was unclear.

2. Certain geographical areas presented persistent classification problems.

3. The so-called Indeterminate (Type 9) classification contained several well-defined subtypes.

An estimate of the scope of the first and second problems was obtained by polling the team of 10 analysts doing the classifications. Each was asked to subjectively rate whether it was easy or difficult to determine the weather pattern type in various parts of the country. Table 5 gives the results. Totals along the right hand side of the table show that the coastal areas and the Pacific Northwest were the most problematic areas. The totals along the bottom of the table show that the most problematic types were $4,5,6,8$ and 9. The distribution within the body of the table suggests that the continental and maritime highs $(4,5,6)$ caused problems along the coasts, while occlusions were difficult to handle in the mountains. The transition of Pacific high pressure regions to Type 4 over land and the transition of continental highs to Types 6 or 9 over the Atlantic or Gulf were sometimes poorly defined (e.g., because of weak gradients). Over the mountains of the western U.S., the inland progression of weakening occlusions and subsequent developments along quasi-stationary fronts on the east slope of the Rockies frequently presented difficulties because of the inapplicability of the Polar Front Model.

The third problem arose largely from the inapplicability of the Polar Front Model to weather patterns frequently found in various parts of the country. Table 6 describes commonly found synoptic features and their preferred geographical locations and seasons of occurrence that were 
TABLE 6. Synoptic Features Classified as Type 9: Indeterminate

\begin{tabular}{|c|c|c|}
\hline Synoptic Feature & Preferred Location & $\begin{array}{l}\text { Preferred } \\
\text { Season(s) }\end{array}$ \\
\hline Thermal lows and troughs & Mexico, CA, AZ, NV & $\begin{array}{l}\text { Summer } \\
\text { Spring } \\
\text { Autumn }\end{array}$ \\
\hline Dry lee troughs & NM, CO, WY, MT & ? \\
\hline $\begin{array}{l}\text { I11-defined surface lows (no fronts) } \\
\text { associated with intense upper-air } \\
\text { disturbances }\end{array}$ & Southwestern U.S. & $\begin{array}{l}\text { Winter } \\
\text { Spring }\end{array}$ \\
\hline $\begin{array}{l}\text { Ill-defined surface lows associated with } \\
\text { very old weak occlusions (no fronts) } \\
\text { well to the rear of vigorous new } \\
\text { occlusions }\end{array}$ & NW and NE U.S. & $\begin{array}{l}\text { Winter } \\
\text { Spring }\end{array}$ \\
\hline $\begin{array}{l}\text { Tropical waves, tropical depressions, } \\
\text { tropical storms and hurricanes }\end{array}$ & SE U.S., Gulf Coast & $\begin{array}{l}\text { Summer } \\
\text { Autumn }\end{array}$ \\
\hline Modified polar continental air masses & Western Atlantic & $\begin{array}{l}\text { Winter } \\
\text { Spring }\end{array}$ \\
\hline Maritime polar air masses & Eastern Pacific & $\begin{array}{l}\text { Winter } \\
\text { Spring }\end{array}$ \\
\hline Maritime Pacific High & Eastern Pacific & $\begin{array}{l}\text { Summer } \\
\text { Autumn }\end{array}$ \\
\hline \multicolumn{3}{|c|}{$\begin{array}{l}\text { classified as Type 9: Indeterminate. Thermal lows and troughs over the } \\
\text { southwestern United States and northern Mexico were, by far, the most commonly } \\
\text { found feature that was classified as Type } 9 \text {. These are primarily warm-season } \\
\text { features. The least occurring features were the tropical waves, disturbances, } \\
\text { storms and hurricanes that infrequently visit the southeastern Atlantic coast, } \\
\text { Florida and the Gulf Coast in the warmer months. In the fall, weather systems } \\
\text { and storm tracks undergo the transition from summer to winter. Dry troughs to } \\
\text { the lee of the Rockies and maritime polar air masses along the west coast } \\
\text { often were classed as indeterminate. Ill-defined surface lows were a common } \\
\text { winter classification problem over the southwest. Modified continental polar } \\
\text { air masses moving off the east coast were noted as a spring time problem. } \\
\text { Anticyclonic features, associated with maritime polar or modified continental }\end{array}$} \\
\hline
\end{tabular}


polar air masses, that could not be classified except as Type 9, were far fewer than the number of cyclonic features, which did not fit the Polar Front Mode1. 


\section{RECOMMENDATIONS}

Classification of surface weather patterns has revealed some interesting regional features. However, in spite of the care taken to define each weather pattern type in an objective and operational manner, many weather situations did not fall into the classification scheme based on the Polar Front Model. Definition of additional weather pattern types would have reduced the number of indeterminate classifications. Yet a subjective manual analysis, which is a tedious task, would still encounter situations that defied classification. Future activity in weather pattern typing, if undertaken, should examine the use of numerical objective analysis techniques based on the distribution of surface pressure and weather features (personal communication with Lester and Burton in 1982).

The statistical analyses presented here have examined the climatolngy of weather pattern occurrence. Many other phenomena may show correlations with the weather pattern types used here. For instance, Barchet and Davis (1983) used a weather pattern climatology to estimate the long-term mean wind speed from a short period of record. Although this work did not indicate an improvement over other available techniques by using the weather pattern climatology, the extension of the weather pattern climatology from the the Great Plains (Barchet 1982) to that of the United States was considered a valuable study for many other applications.

Often it is necessary to infer the long-term (climatological) value of a weather-dependent quantity from a relatively short period of observation. There is then always the risk that the period of observation does not adequately represent the climatology of the measurement location. The weather pattern climatology presented here offers a means by which the period of observation can be compared against the long-term climatology of weather patterns at the point of interest. It may be possible, if necessary, to adjust the observed parameter for any bias in weather pattern occurrence (as was done by Barchet and Davis (1982) for wind speed).

However, classification of weather patterns is a subjective skill that requires special training as well as experience in general synoptic 
meteorology. Comparison with the climatology presented here will require that the procedures given in Appendix A be carefully followed. 


\section{$\underline{\text { REFERENCES }}$}

Barchet, W. R. 1982. A Weather Pattern Climatology of the Great Plains and the Related Wind Regime. PNL-4330, Pacific Northwest Laboratory, Richland, Washington.

Barchet, W. R. and W. E. Davis. 1983. Estimating Long-Term Mean Wind From Short-Term Wind Data. PNL-4785, Pacific Northwest Laboratory, Richland, Washington. 


$$
\text { - }
$$


APPENDIX A 
APPENDIX A

\section{GUIDELINES FOR THE OPERATIONAL CLASSIFICATION OF WEATHER PATTERNS}

In preparation for the operational classification of weather pattern types, the criteria for designating each type were expanded and refined with the objective to provide guidelines to classification which would standardize the procedure and produced the fewest indeterminate classifications. These guidelines were based on the following assumptions:

i. The Polar Front Model (with modifications for the peculiarities of the North American Continent) describes the general synoptic structure of all mid- and high-latitude disturbances.

ii. Weather map features of Synoptic Scale dimensions are of major importance. "Synoptic Scale" features are defined here as those with areas greater than one grid square ( $3^{\circ}$ latitude by $4^{\circ}$ longitude).

iii. Surface windflow patterns of synoptic dimensions are related to the topography of the sea-level pressure field (i.e., the configuration of isobars) via the geostrophic (gradient) wind relationship.

iv. On the Synoptic Scale there is a strong relation between the isobaric pattern and the presence of moisture, clouds and precipitation.

TYPE 1: WARM SECTOR (OPEN WAVE OR OCCLUSION)

a. The largest portion of the warm air mass that is encompassed by the cold front, the warm front, and

The line from the "bow" of the warm front to the "bow" of the cold front (1 ine "a") in Figure A.1, or

The 1 ine from the point at which the warm front becomes a stationary front to the point at which the cold front becomes a stationary front (1 ine "b" in Figure A.1), not to extend beyond the COL on either front, (if the front is truncated, it may be extended to the COL for classification purposes) or 


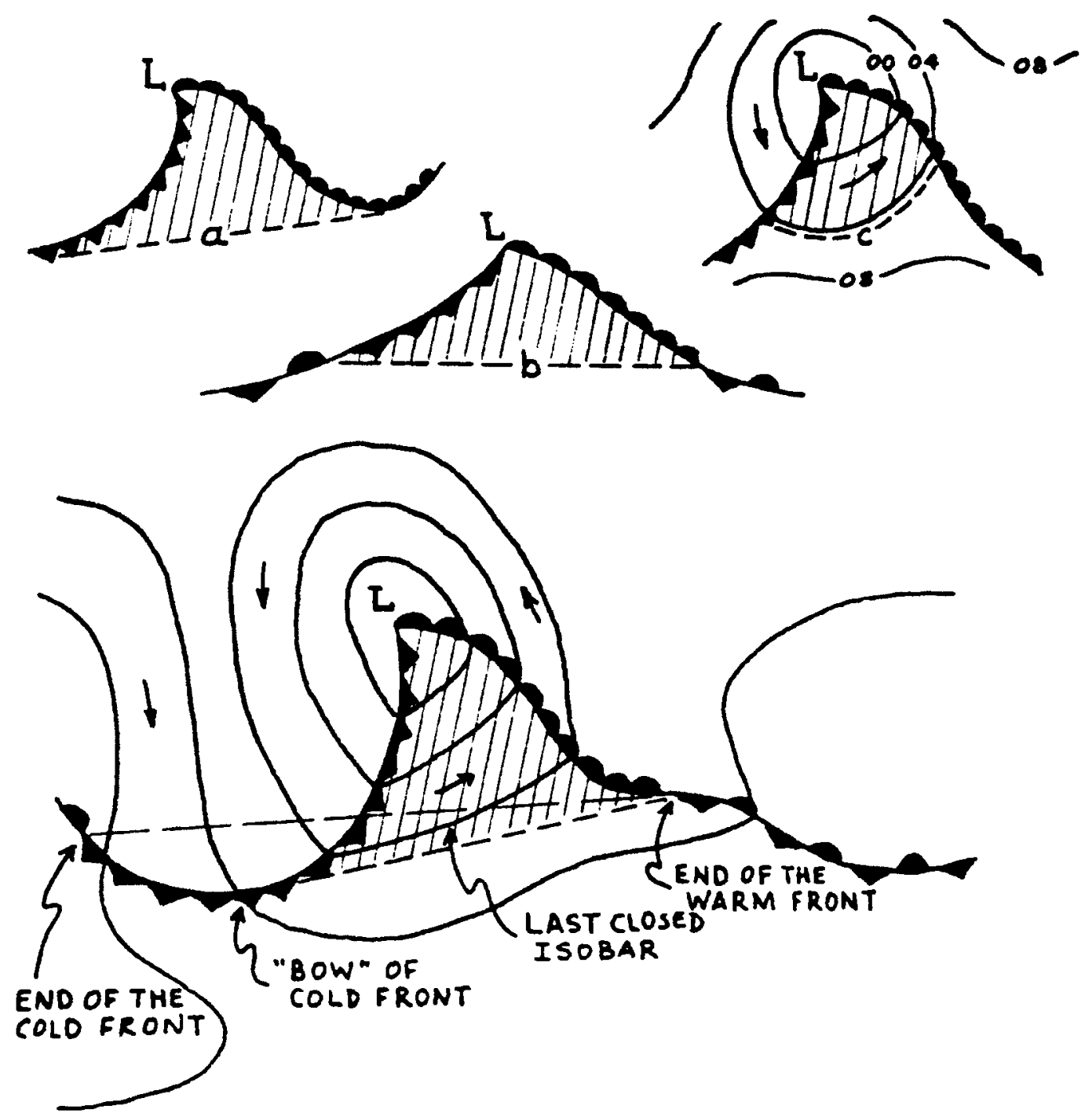

FIGURE A-1. Type 1 (Warm Sector): For Open Wave and Occluded Cyclones. Regions selected are shown as hatched areas. Rules are iliustrated for individual cases (top) and in combination (bottom). Short arrows between isobars represent directions of geostrophic (gradient) wind.

The last closed isobar in the warm sector (1ine "c" in Figure A.1) that has curvature which is straight, cyclonic or slightly anticyclonic, or A combination of the above to maximize the area.

b. In the case of double fronts from the same low, only the southernmost fronts will be used for classification purposes. 
c. In ambiguous situations, the warm sector may be better defined by geostrophic winds with a south-and/or west-component, indications of high humidity (e.g., $\equiv, \oplus, \mathbb{D},,, \bullet$ ) and/or falling pressure.

TYPE 2: AHEAD OF THE WARM FRONT (OPEN WAVE OR OCCLUSION)

a. The Type 2 area is encompassed by the warm front, and lines "a" and "b" in Figure A.2. Line "a" begins at the end of the warm front and extends counterclockwise around the low to line "b" which extends from the apex of the warm sector, through the points on the cyclonically curved isobars which separate easterly and westerly geostrophic flow (usually behind the cyclone).

b. For an occlusion (Figure A.2), line "b" separates southerly from northerly geostrophic flow.

c. For waves with little amplitude, both with one or more closed isobars, line "b" will be arbitrarily drawn at a $45^{\circ}$ angle with the cold front (see Figure A.3).

d. In both the wave and occluded cases, the Type 2 area will encompass primarily cyclonically curved isobars, extending north of the low to the last closed isobar on the edge of the precipitation which ever maximizes the area. If the northern extent of the area is ambiguous according to these guidelines, the COL is the northern limit.

e. In the case of double fronts, use rule (b) under Type 1.

f. In $\mathbf{1 1 1 - d e f i n e d , ~ p r e w a r m - f r o n t a l ~ a r e a s , ~ h i g h - h u m i d i t y ~ i n d i c a t o r s ~}(\oplus,,, \bullet)$ and pressure falls (ahead of the low) should be used as secondary criteria to better delineate the region.

g. Where the warm front is very poorly defined (e.g., with occlusions over the mountains of western U.S.), line "a" will correspond with the last closed isobar ahead of the occluded front. 


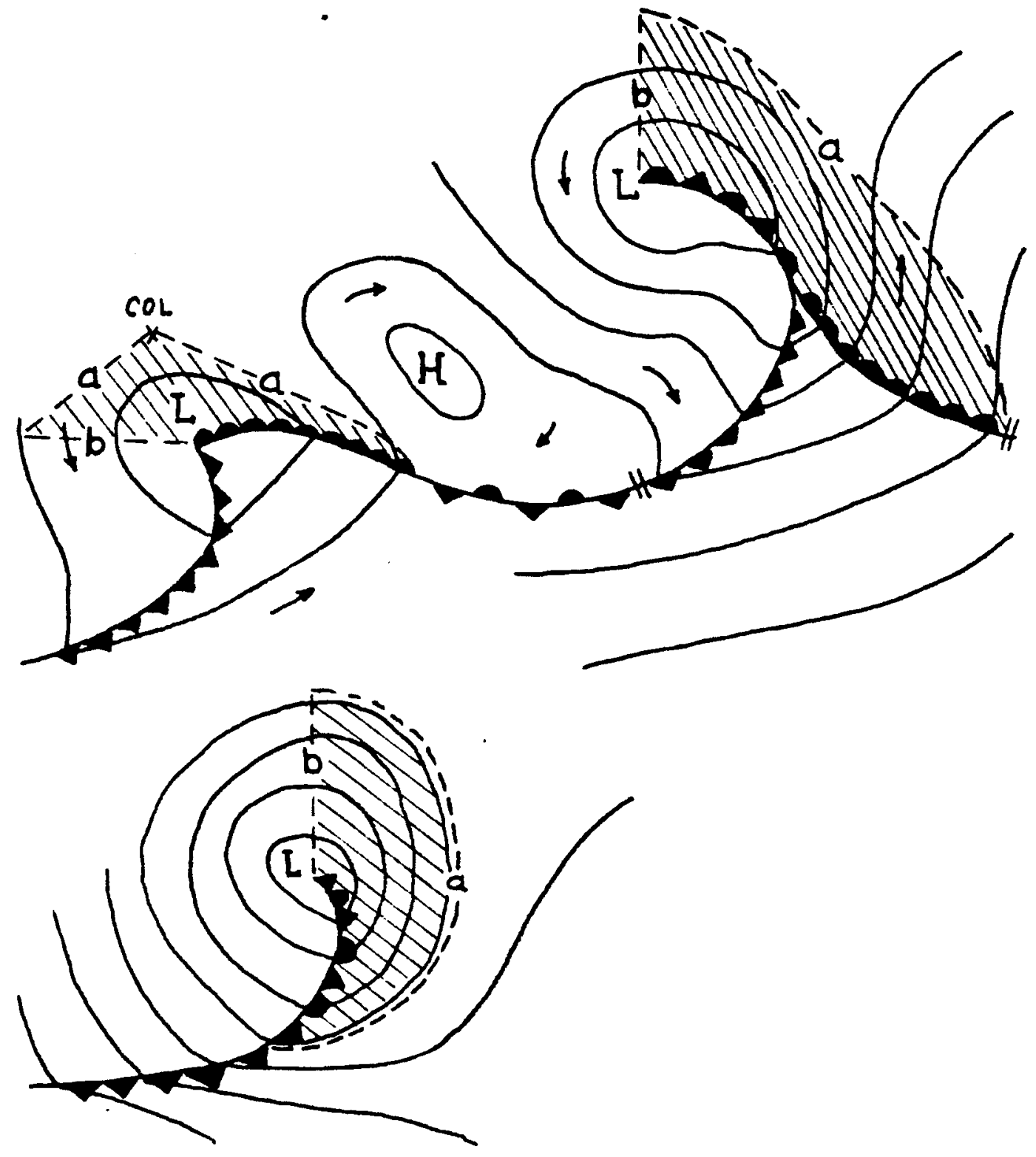

FIGURE A.2. Type 2 (Ahead of Warm Front). For open wave (top left), occlusion (top right), and occlusion with poorly defined warm front (bottom). Selected regions are hatched.

\section{TYPE 3: BEHIND THE COLD FRONT (OPEN WAVE OR OCCLUSION)}

a. For both occlusions and open waves, the Type 3 region is the largest area enclosed by the cold front and the line extending from the apex of the warm sector, through the cold air, to the point at which the cold front becomes a stationary front (Figure A.3). 


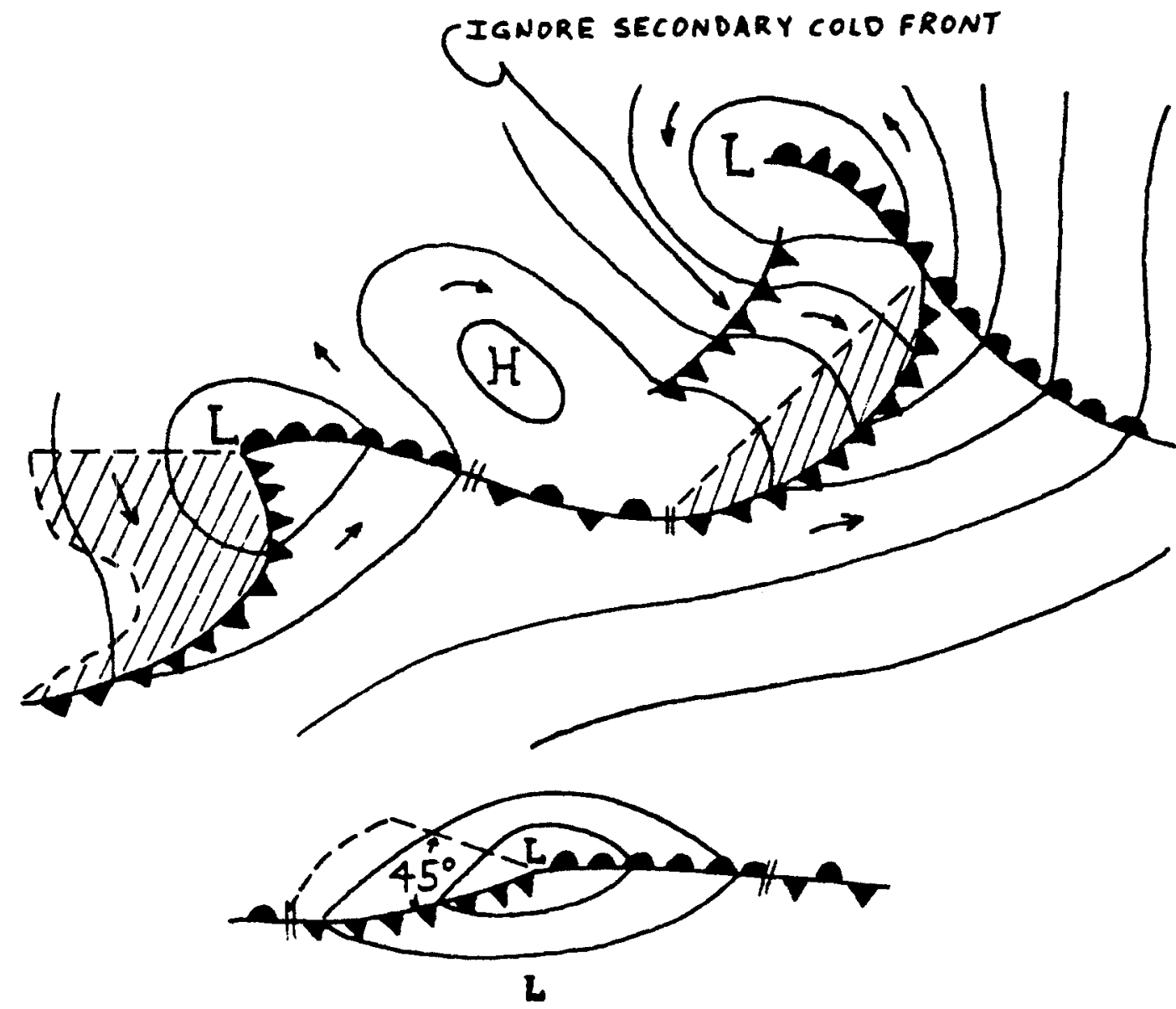

FIGURE A.3. Type 3 (Behind Cold Front). For open wave (upper 1eft), occlusion (upper right), and stable wave (bottom). Selected areas are hatched.

b. For open waves (Figure A.3), the area will include cyclonically curved isobars behind the low that are not included in Type 2, as well as the cyclonically curved isobars immediately behind the front. This area is usually characterized by northwesterly flow (also see Type 8).

c. For occluded cyclones (Figure A.3) the Type 3 will usually be 1 imited to the region immediately behind the cold front.

d. For stable waves (very small amplitude with one or more closed isobars), the boundary of the area will be a line extending into the cold air from the apex of the warm sector. It will make a $45^{\circ}$ angle with the cold 
front, encompass the cyclonic curvature and terminate at the point the cold front becomes stationary (Figure A.3).

e. In cases of double fronts, use rule (b), Type 1.

f. In poorly defined cases, secondary criteria ( $\mathbb{D}, \dot{\nabla}$, pressure rises) will better define the area.

\section{TYPES 4 AND 5: CONTINENTAL HIGH}

a. All areas over land, immediately to the east of the major ridgeline of a continental high will be classified as Type 4 if the isobars are anticyclonically curved and the geostrophic wind has a northerly component (Figure A.4).

b. All areas over land, immediately to the west of the major ridgeline of a continental high will be classified as Type 5 if the isobars are anticyclonically curved and the geostrophic wind has a southerly component (Figure A.4).

c. As long as the major ridgeline of the continental high remains over land, regions over water with northerly or northwesterly flow will be classified as Type 4 (east coast). After the major ridgeline moves over the water, all associated overwater areas with anticyclonic curvature will be classified as either Type 6 or Type 9 (see Types 6 and 9).

d. When a portion of the Pacific High builds over the west coasts, only the region overland will usually be classified as Type 4 (for a northerly component of the geostrophic wind) or Type 5 (for a southerly component).

e. In ambiguous cases, secondary criteria (e.g., $\equiv, \oplus,,, \bullet$, pressure rises) will be used to clarify the pattern.

\section{TYPE 6: MARITIME HIGH}

a. A high pressure area centered over water will be considered as a "maritime" high. 


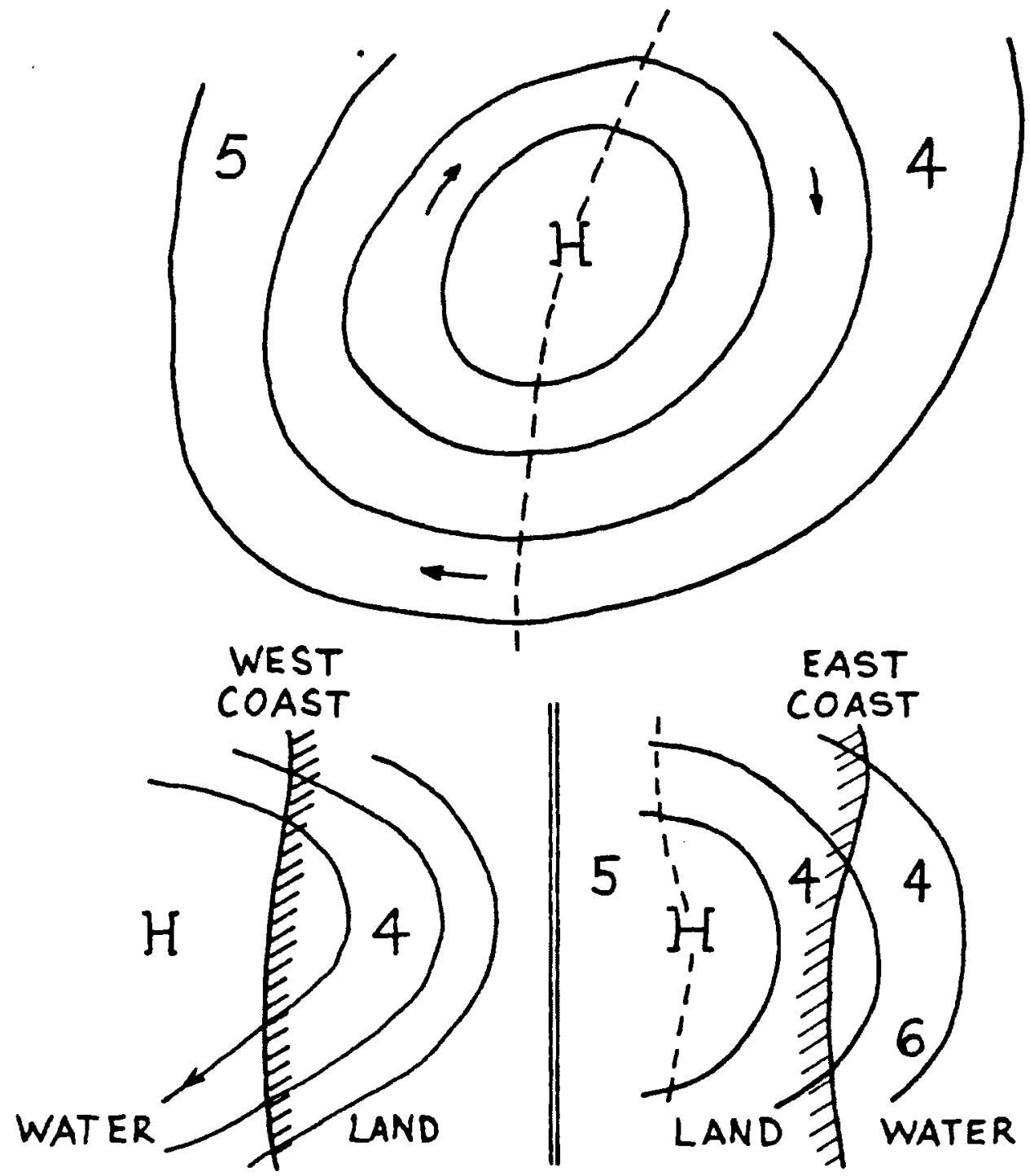

FIGURE A-4. Type 4 (East of a Continental High) and Type 5 (West of a Continental High). West coast and east coast transition types are shown at the bottom. Type 4 regions are hatched. See also Type 6.

b. All over water areas within the maritime high which show anticyclonically curved isobars and an easterly or southerly geostrophic component will be classified as Type 6.

c. Over land, all areas under the influence of the maritime high (anticyclonic curvature and easterly and/or southerly geostrophic wind component) which have indicators or high humidity will be classified as Type 6 . 
d. Type 6 may not exist near the west coast.

e. After the center or the major ridgeline of a continental high moves over water, regions of the high which satisfy (b) and (c) above will be classified as Type 6 .

\section{TYPE 7: STATIONARY FRONT}

a. All quasi-stationary (i.e., slow moving warm or cold, or stationary) fronts will be designated as Type 7.

b. The Type 7 classification encompasses the region around the quasistationary front which is characterized by cyclonic shear and/or cyclonic curvature of the isobars and/or precipitation.

c. Regions of frontogenesis and frontolysis will usually be included in this classification.

d. Nonfrontal, lee-of-the-mountain troughs which show precipitation and/or extensive cloudiness will be classified as Type 7 .

\section{TYPE 8: OCCLUDED FRONT AND REGIONS WEST OF OCCLUDED LOW}

a. The region of cyclonic curvature and precipitation behind the occluded front and low, will be designated as Type 8 (Figure A.5).

b. Type 8 is encompassed by the line ("a" in Figure A.5) which extends from the apex of the warm sector, behind the occlusion to the intersection of the last closed isobar around the low and a line that separates southeasterly from northeasterly geostrophic flow (1 ine "b" in Figure A.5).

c. In ambiguous cases, Type 8 may be distinguished by northerly geostrophic flow, overcast skies and precipitation.

\section{TYPE 9: INDETERMINE}

Type 9 is the "catch all" classification which generally includes synoptic conditions not covered by Types 1-8. 


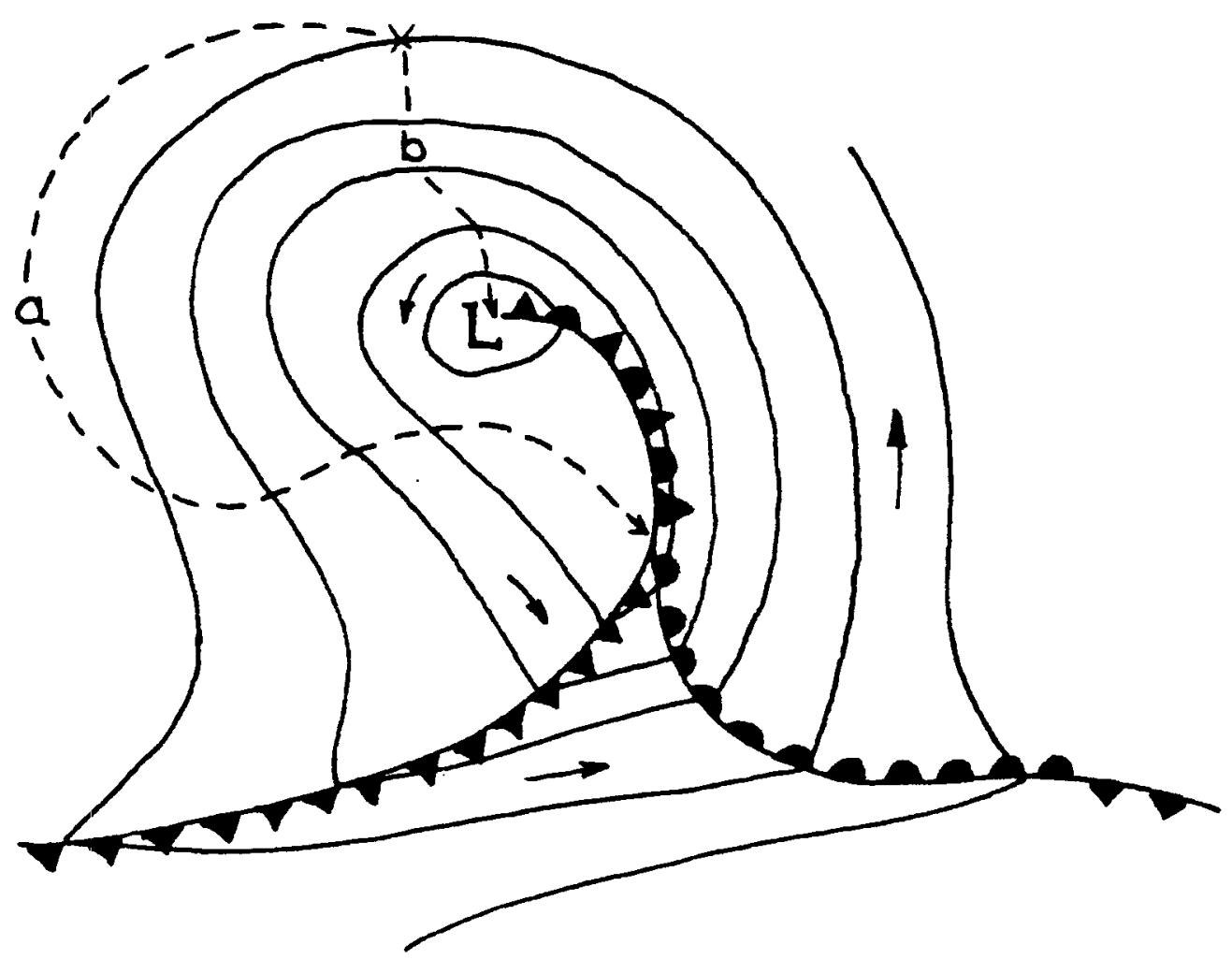

FIGURE A.5. Type 8 (Occluded Front and Region West of Occluded Low). Selected region is hatched.

\section{Additional Guidelines}

An evaluation of the application of the above guidelines to the operational, routine analysis of weather pattern types leads to the formulation of a several additional guidelines that help promote consistency among analysts:

i. Adhere to the principles of continuity both in the interpretations and in the classifications of synoptic circulation systems.

ii. Minimize "9" (indeterminate) areas wherever possible.

iii. Where two classifications are equally possible, select the numerically lower classification.

iv. Use upper air (500 mb) to check continuity and to determine the vertical structure of systems (especially occlusions).

v. Accept NWS analyses except in cases with obvious analysis errors or breaks in continuity. 


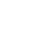


APPENDIX B 


\section{WEATHER PATTERN TYPE DATA BASE}

The time series of weather pattern type for the 120 points shown in Figure 1 are maintained on a 1/2-inch, 9-track, 1600 bpi, ASCII-coded, unlabeled magnetic tape. Forty points in the $16 \times 10$ grid at which no weather pattern type were coded 0 . The grid point data are reported in full in a separate volume.

Each logical record contains date and weather patterns for a latitude band consisting of 16 points $\left(126^{\circ} \mathrm{W}\right.$ to $66^{\circ} \mathrm{W}$ at $4^{\circ}$ longitude intervals). The format for each logical record of 38 characters is given in Table B.1. Logical records are blocked to contain 1 day's data.

TABLE B.1. Logical Record Format for U.S.A. Weather Pattern Data Tape

\begin{tabular}{|c|c|c|}
\hline Field & Width & Description \\
\hline 1 & 2 & Year, e.g., $1955=55$ \\
\hline 2 & 2 & Month, e.g., May $=05$ \\
\hline 3 & 2 & Day of month, e.g., 02 \\
\hline 4 & 2 & Map time, e.g., $12 Z=12$ \\
\hline 5 & 2 & Weather pattern at $126^{\circ} \mathrm{W}$ \\
\hline - & - & - \\
\hline$\cdot$ & $\cdot$ & - \\
\hline - & - & - \\
\hline 19 & 2 & Weather pattern at $66^{\circ} \mathrm{W}$ \\
\hline
\end{tabular}

The ten logical records within a block are ordered from north $\left(51^{\circ} \mathrm{N}\right)$ to south $\left(24^{\circ} \mathrm{N}\right)$ at $3^{\circ}$ latitude intervals. The format of each block of 380 characters is given in Table B.2.

A sample of the data tape contents is shown in Table B.3. 
TABLE B.2. B1ock Format for U.S.A. Weather Pattern Data Tape

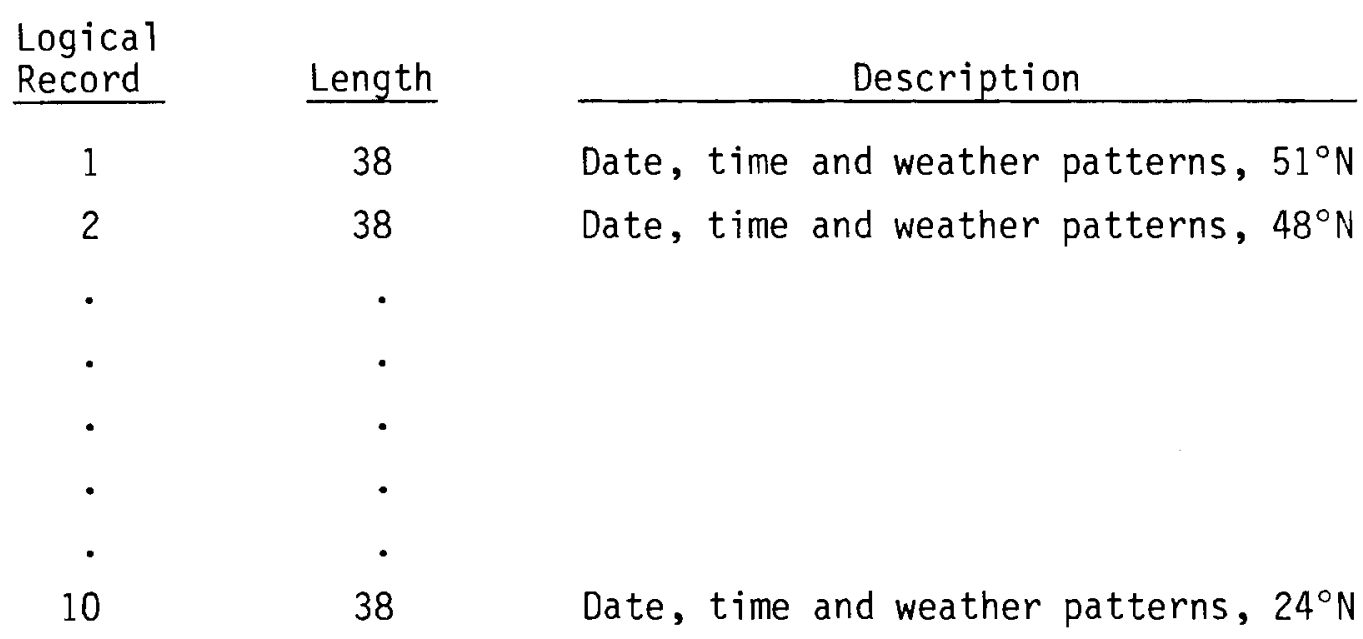

TABLE B.3. Sample of the U.S. Weather Pattern Data Tape Content

$\begin{array}{lllllllllllllllll}76122312 & 9 & 9 & 9 & 5 & 5 & 4 & 5 & 7 & 7 & 6 & 6 & 6 & 6 & 6 & 0 & 0\end{array}$

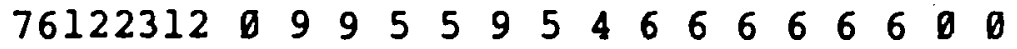
76122312000999999957777766606000

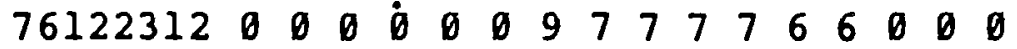

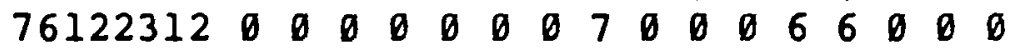

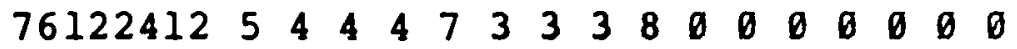

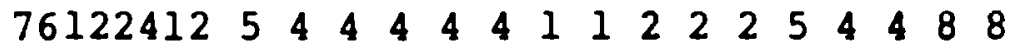
$\begin{array}{lllllllllllllllllll}761224412 & 5 & 5 & 4 & 4 & 4 & 4 & 9 & 2 & 2 & 5 & 5 & 5 & 4 & 4 & 8 & 8\end{array}$

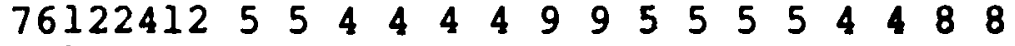

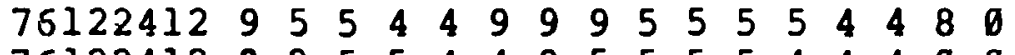

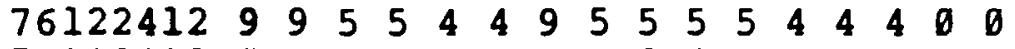

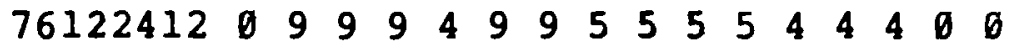
$\begin{array}{lllllllllllllllll}76122412 & 0 & 0 & 9 & 9 & 9 & 9 & 6 & 5 & 5 & 5 & 5 & 4 & 4 & 0 & 0 & 0\end{array}$

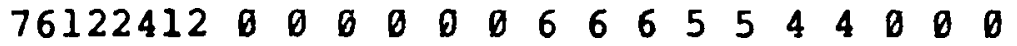

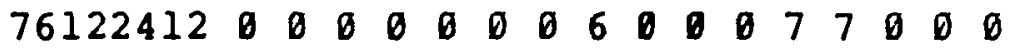

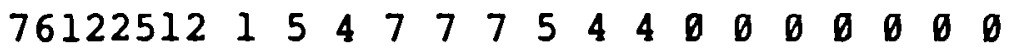
$\begin{array}{llllllllllllllllll}76122512 & 1 & 5 & 4 & 4 & 4 & 7 & 7 & 7 & 4 & 4 & 4 & 3 & 2 & 2 & 5 & 4\end{array}$ $\begin{array}{llllllllllllllllll}76122512 & 1 & 5 & 5 & 4 & 4 & 4 & 4 & 7 & 7 & 4 & 3 & 1 & 1 & 5 & 5 & 4\end{array}$

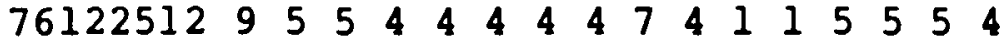

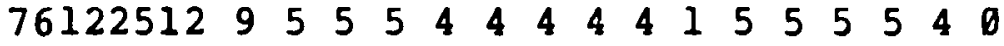
$\begin{array}{llllllllllllllllll}76122512 & 9 & 9 & 5 & 5 & 4 & 4 & 4 & 4 & 7 & 6 & 6 & 6 & 5 & 5 & 0 & 0\end{array}$ 
APPENDIX C 
. 


\section{PERCENTAGE FREQUENCY OF WEATHER PATTERN OCCURRENCE}

The data listings in this appendix present the percentage frequency of weather pattern occurrence in five sets of tables:

$$
\begin{aligned}
& \text { Annual - al1 months } \\
& \text { Winter - December, January, February } \\
& \text { Spring - March, Apri1, May } \\
& \text { Summer - June, July, August } \\
& \text { Autumn - September, October, November. }
\end{aligned}
$$

Following the annual and seasonal data are 10 sets of yearly frequency of occurrence, one for each year in the period 1969 to 1978. At the end of these tables is the final set on the variability of the yearly frequency of occurrence. This is expressed as 100 times the standard deviation of the 10 yearly means divided by the annual mean frequency.

All of the tables are presented by latitude band across the region. The 10 bands are presented from north to south. Seventeen columns of data are presented in each band. The first column denotes the weather pattern type. The other 16 columns are headed by the longitude of the grid point for which the percentage frequency of occurrence applies. 
Annuel

51 Dearees North Laticude

\begin{tabular}{|c|c|c|c|c|c|c|c|c|c|c|c|c|c|c|c|c|}
\hline \multirow[t]{2}{*}{ VIPT } & \multicolumn{16}{|c|}{ Nearees } \\
\hline & 126 & 122 & 118 & 114 & 110 & 106 & 102 & 98 & 94 & 90 & 86 & A? & 78 & 74 & 70 & 66 \\
\hline $\begin{array}{l}1 \\
2 \\
3 \\
4 \\
5 \\
6 \\
7 \\
8 \\
9\end{array}$ & $\begin{array}{r}1.3 \\
16.5 \\
1.4 \\
17.5 \\
15.5 \\
0.0 \\
6.4 \\
7.7 \\
33.7\end{array}$ & $\begin{array}{r}1.0 \\
11.5 \\
1.3 \\
23.1 \\
23.2 \\
0.0 \\
9.5 \\
6.0 \\
25.3\end{array}$ & $\begin{array}{r}1.2 \\
7.5 \\
1.8 \\
32.0 \\
18.4 \\
0.0 \\
13.9 \\
6.8 \\
18.5\end{array}$ & $\begin{array}{r}2.2 \\
6.5 \\
2.2 \\
27.8 \\
18.6 \\
0.0 \\
17.4 \\
7.7 \\
17.5\end{array}$ & $\begin{array}{r}2.5 \\
8.3 \\
2.2 \\
25.1 \\
22.7 \\
0.0 \\
14.3 \\
8.9 \\
15.9\end{array}$ & $\begin{array}{r}2.2 \\
10.8 \\
2.3 \\
24.5 \\
26.0 \\
0.0 \\
11.8 \\
9.5 \\
12.8\end{array}$ & $\begin{array}{r}2.7 \\
10.4 \\
2.7 \\
26.2 \\
26.7 \\
0.0 \\
9.8 \\
10.9 \\
10.7\end{array}$ & $\begin{array}{r}2.5 \\
12.1 \\
2.8 \\
28.2 \\
25.1 \\
0.0 \\
8.1 \\
12.2 \\
9.0\end{array}$ & $\begin{array}{r}2.7 \\
13.6 \\
2.8 \\
27.6 \\
25.8 \\
0.0 \\
6.8 \\
12.5 \\
8.1\end{array}$ & 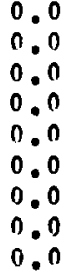 & $\begin{array}{l}0.0 \\
0.0 \\
0.0 \\
0.0 \\
0.0 \\
0.0 \\
0.0 \\
0.0 \\
0.00 \\
0.0 \\
0.0\end{array}$ & $\begin{array}{l}0.0 \\
0.0 \\
0.0 \\
0.0 \\
0.0 \\
0.0 \\
0.0 \\
0.00 \\
0.0 \\
0.0\end{array}$ & 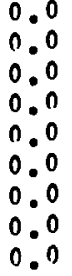 & 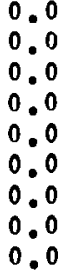 & $\begin{array}{l}0.0 \\
0.0 \\
0.0 \\
0.0 \\
0.0 \\
0.0 \\
0.0 \\
0.0 \\
0.0\end{array}$ & $\begin{array}{l}0.0 \\
0.0 \\
0.0 \\
0.0 \\
0.0 \\
0.0 \\
0.0 \\
0.0 \\
0.0\end{array}$ \\
\hline
\end{tabular}

48 Dearees North Lotitude

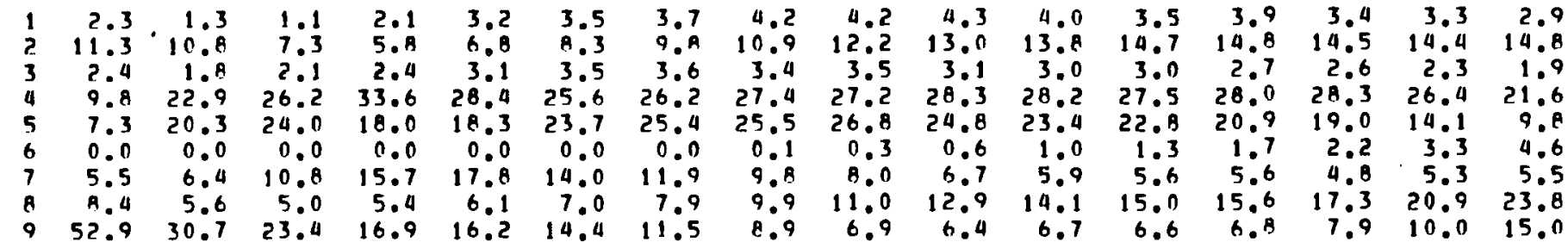

45 Dearees North Latftude

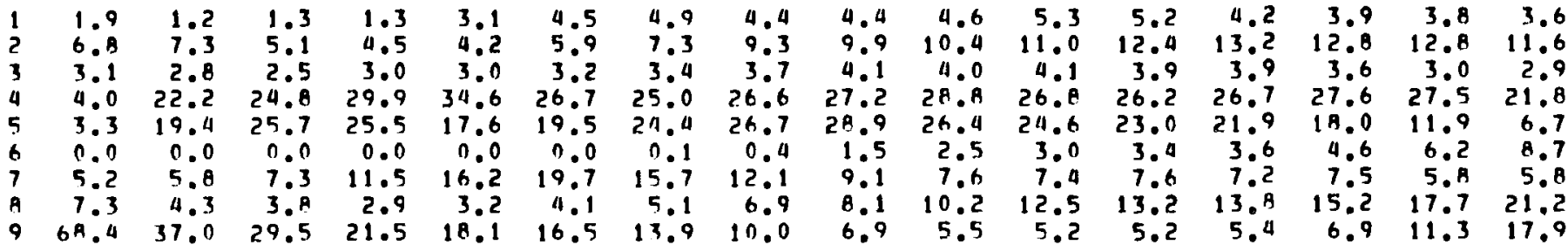

42 Dearees North Latitude

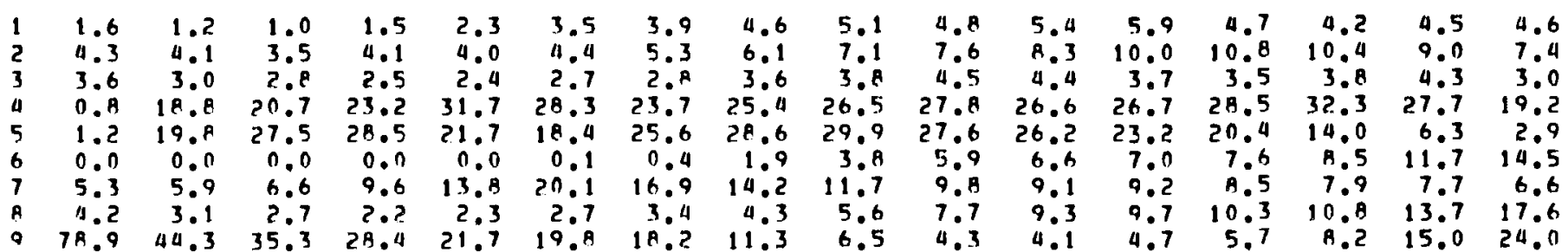


Annuel

39 Degrees North Latitude

\begin{tabular}{|c|c|c|c|c|c|c|c|c|c|c|c|c|c|c|c|c|}
\hline WPT & 126 & 122 & 118 & 114 & 110 & $106^{\circ}$ & $\begin{array}{c}\text { earees } \\
102\end{array}$ & $\begin{array}{r}\text { We } 8 \mathrm{t} \\
98\end{array}$ & $\begin{array}{c}\text { Longlt } \\
94\end{array}$ & $\begin{array}{l}\text { tde } \\
90\end{array}$ & B6 & 82 & 78 & 74 & 70 & 66 \\
\hline $\begin{array}{l}1 \\
2 \\
3 \\
4 \\
5 \\
6 \\
7 \\
8 \\
9\end{array}$ & $\begin{array}{r}1.2 \\
1.8 \\
2.8 \\
0.2 \\
0.2 \\
0.0 \\
4.9 \\
2.7 \\
86.2\end{array}$ & $\begin{array}{r}0.8 \\
2.1 \\
2.7 \\
10.3 \\
12.2 \\
0.0 \\
5.4 \\
1.7 \\
64.8\end{array}$ & $\begin{array}{r}0.7 \\
2.5 \\
2.2 \\
15.1 \\
26.5 \\
0.0 \\
5.4 \\
2.1 \\
45.5\end{array}$ & $\begin{array}{r}1.7 \\
3.0 \\
2.1 \\
16.0 \\
31.0 \\
0.0 \\
7.1 \\
1.8 \\
37.4\end{array}$ & $\begin{array}{r}1.9 \\
2.8 \\
1.9 \\
22.5 \\
27.6 \\
0.1 \\
10.0 \\
1.8 \\
31.4\end{array}$ & $\begin{array}{r}2.4 \\
3.3 \\
2.0 \\
30.9 \\
17.6 \\
0.2 \\
17.0 \\
2.0 \\
24.6\end{array}$ & $\begin{array}{r}3.4 \\
4.0 \\
2.9 \\
19.4 \\
25.0 \\
1.2 \\
19.4 \\
2.1 \\
22.6\end{array}$ & $\begin{array}{r}9.4 \\
5.0 \\
3.2 \\
21.9 \\
28.8 \\
5.7 \\
16.0 \\
2.9 \\
12.2\end{array}$ & $\begin{array}{r}5.0 \\
5.3 \\
3.5 \\
24.0 \\
28.8 \\
10.5 \\
13.4 \\
4.0 \\
5.7\end{array}$ & $\begin{array}{r}4.6 \\
5.4 \\
4.7 \\
26.3 \\
26.3 \\
13.0 \\
11.7 \\
4.4 \\
3.7\end{array}$ & $\begin{array}{r}5.3 \\
6.2 \\
4.1 \\
26.9 \\
23.9 \\
13.7 \\
11.2 \\
5.1 \\
3.5\end{array}$ & $\begin{array}{r}5.5 \\
7.3 \\
3.8 \\
28.2 \\
21.5 \\
14.1 \\
10.3 \\
0.8 \\
4.5\end{array}$ & $\begin{array}{r}4.9 \\
7.7 \\
4.0 \\
31.4 \\
15.5 \\
13.8 \\
10.4 \\
5.5 \\
6.8\end{array}$ & $\begin{array}{r}5.3 \\
6.2 \\
9.1 \\
31.8 \\
6.4 \\
17.2 \\
9.4 \\
7.0 \\
12.7\end{array}$ & $\begin{array}{r}5.9 \\
4.8 \\
4.2 \\
22.6 \\
2.2 \\
20.2 \\
8.2 \\
10.0 \\
22.3\end{array}$ & $\begin{array}{ll}0 & 0 \\
0 & 0 \\
0.0 & 0 \\
0.0 & 0 \\
0.0 & 0 \\
0.0 & 0 \\
0.0 & 0 \\
0.0 & 0 \\
0.0 & 0\end{array}$ \\
\hline
\end{tabular}

36 Degrees North Letitude

$\begin{array}{rrrrrrrrrrr}2.0 & 3.4 & 3.8 & 3.5 & 4.3 & 4.3 & 3.9 & 4.4 & 4.8 & 0.0 & 0.0 \\ 1.6 & 2.3 & 2.6 & 3.4 & 3.3 & 4.4 & 4.6 & 3.8 & 2.6 & 0.0 & 0.0 \\ 2.0 & 2.5 & 2.1 & 3.1 & 3.0 & 3.4 & 3.8 & 3.8 & 4.2 & 0.0 & 0.0 \\ 26.6 & 19.8 & 18.8 & 21.0 & 24.8 & 26.0 & 27.4 & 29.7 & 23.6 & 0.0 & 0.0 \\ 17.8 & 24.7 & 29.8 & 27.1 & 22.9 & 19.6 & 15.9 & 7.9 & 2.3 & 0.0 & 0.0 \\ 0.5 & 9.8 & 16.0 & 22.3 & 25.1 & 25.5 & 24.8 & 26.0 & 29.3 & 0.0 & 0.0 \\ 12.0 & 16.6 & 13.8 & 12.7 & 11.4 & 11.1 & 11.9 & 12.1 & 11.7 & 0.0 & 0.0 \\ 1.4 & 1.3 & 1.5 & 2.2 & 2.0 & 2.0 & 2.3 & 2.9 & 3.9 & 0.0 & 0.0 \\ 36.0 & 29.5 & 11.7 & 4.7 & 3.3 & 3.6 & 5.3 & 9.4 & 17.6 & 0.0 & 0.0\end{array}$

33 Dearees North Latitude

\begin{tabular}{|c|c|c|c|c|c|c|c|c|c|c|c|c|c|c|c|}
\hline $\begin{array}{l}1 \\
2 \\
3 \\
4 \\
5 \\
6 \\
7 \\
8\end{array}$ & $\begin{array}{ll}0 & 0 \\
0.0 & 0 \\
0.0 & 0 \\
0.0 & 0 \\
0.0 & 0 \\
0.0 & 0 \\
0.0 & 0 \\
0.0 & 0 \\
0 & 0\end{array}$ & $\begin{array}{r}0.4 \\
0.3 \\
1.2 \\
0.1 \\
0.1 \\
0.0 \\
1.8 \\
0.6 \\
95.5\end{array}$ & $\begin{array}{r}0.4 \\
0.4 \\
1.2 \\
4.8 \\
4.1 \\
0.0 \\
2.5 \\
0.5 \\
86.1\end{array}$ & $\begin{array}{r}0.6 \\
0.5 \\
1.5 \\
5.7 \\
12.1 \\
0.0 \\
3.0 \\
0.5 \\
76.1\end{array}$ & $\begin{array}{r}0.9 \\
0.6 \\
0.9 \\
8.8 \\
25.2 \\
0.2 \\
3.3 \\
0.7 \\
59.3\end{array}$ & $\begin{array}{r}1.5 \\
0.8 \\
1.5 \\
17.7 \\
15.4 \\
1.2 \\
11.0 \\
0.6 \\
50.4\end{array}$ & $\begin{array}{r}2.1 \\
1.3 \\
2.1 \\
9.5 \\
25.3 \\
11.6 \\
13.5 \\
0.7 \\
33.9\end{array}$ & $\begin{array}{r}2.5 \\
1.0 \\
1.6 \\
15.2 \\
25.7 \\
30.6 \\
12.3 \\
0.7 \\
10.0\end{array}$ & $\begin{array}{r}2.7 \\
1.5 \\
2.2 \\
19.1 \\
20.8 \\
37.3 \\
10.9 \\
0.9 \\
4.6\end{array}$ & $\begin{array}{r}2.8 \\
2.0 \\
2.0 \\
22.5 \\
17.0 \\
39.0 \\
9.9 \\
0.9 \\
3.9\end{array}$ & $\begin{array}{r}3.2 \\
2.5 \\
2.5 \\
23.4 \\
13.8 \\
38.1 \\
10.8 \\
0.7 \\
4.9\end{array}$ & $\begin{array}{r}3.0 \\
2.4 \\
3.0 \\
24.7 \\
8.5 \\
37.9 \\
12.9 \\
0.8 \\
7.0\end{array}$ & $\begin{array}{r}3.3 \\
1.2 \\
2.9 \\
22.8 \\
3.2 \\
40.7 \\
12.8 \\
1.1 \\
12.0\end{array}$ & $\begin{array}{r}3.4 \\
1.0 \\
2.9 \\
14.5 \\
0.5 \\
43.0 \\
11.8 \\
2.1 \\
20.9\end{array}$ & $\begin{array}{l}0.0 \\
0.0 \\
0.0 \\
0.0 \\
0.0 \\
0.0 \\
0.0 \\
0.0 \\
0.0\end{array}$ \\
\hline
\end{tabular}

30 Deorees North Latitude

\begin{tabular}{|c|c|c|c|c|c|c|c|c|c|c|c|c|c|c|c|c|}
\hline $\begin{array}{l}1 \\
2 \\
3 \\
4 \\
5 \\
6 \\
7 \\
8 \\
9\end{array}$ & $\begin{array}{l}0.0 \\
0.0 \\
0.0 \\
0.0 \\
0.0 \\
0.0 \\
0.0 \\
0.0 \\
0.0\end{array}$ & $\begin{array}{l}0.0 \\
0.0 \\
0.0 \\
0.0 \\
0.0 \\
0.0 \\
0.0 \\
0.0 \\
0.0 \\
0.0\end{array}$ & $\begin{array}{r}0.1 \\
0.0 \\
0.8 \\
0.5 \\
0.1 \\
0.0 \\
1.1 \\
0.1 \\
97.3\end{array}$ & $\begin{array}{r}0.2 \\
0.1 \\
0.9 \\
2.8 \\
1.5 \\
0.0 \\
1.5 \\
0.2 \\
92.8\end{array}$ & $\begin{array}{r}0.6 \\
0.3 \\
0.6 \\
3.0 \\
4.8 \\
0.1 \\
2.2 \\
0.2 \\
B B .3\end{array}$ & $\begin{array}{r}1.1 \\
0.4 \\
0.7 \\
4.2 \\
7.9 \\
0.6 \\
7.0 \\
0.2 \\
78.0\end{array}$ & $\begin{array}{r}1.1 \\
0.7 \\
0.4 \\
3.7 \\
23.5 \\
16.9 \\
10.1 \\
0.2 \\
43.2\end{array}$ & $\begin{array}{r}0.9 \\
0.7 \\
1.2 \\
12.8 \\
18.3 \\
46.5 \\
9.7 \\
0.1 \\
9.8\end{array}$ & $\begin{array}{r}1.4 \\
1.0 \\
1.8 \\
17.5 \\
9.3 \\
53.3 \\
9.8 \\
0.2 \\
5.7\end{array}$ & $\begin{array}{r}1.9 \\
1.1 \\
1.8 \\
17.6 \\
7.1 \\
54.4 \\
10.4 \\
0.2 \\
5.5\end{array}$ & $\begin{array}{r}2.1 \\
1.2 \\
1.3 \\
18.3 \\
6.0 \\
54.4 \\
10.4 \\
0.1 \\
6.1\end{array}$ & $\begin{array}{r}1.7 \\
0.9 \\
1.6 \\
18.1 \\
3.6 \\
55.9 \\
9.9 \\
0.1 \\
0.1\end{array}$ & $\begin{array}{r}1.9 \\
0.4 \\
1.6 \\
13.9 \\
0.8 \\
57.6 \\
9.9 \\
0.3 \\
13.6\end{array}$ & $\begin{array}{l}0.0 \\
0.0 \\
0.0 \\
0.0 \\
0.0 \\
0.0 \\
0.0 \\
0.0 \\
0.0 \\
0.0\end{array}$ & $\begin{array}{ll}0.0 & 0 \\
0.0 & 0 \\
0.0 & 0 \\
0.0 & 0 \\
0.0 & 0 \\
0.0 & 0 \\
0.0 & 0 \\
0.0 & 0 \\
0.0 & 0\end{array}$ & $\begin{array}{ll}0 & 0 \\
0 & 0 \\
0.0 & 0 \\
0.0 & 0 \\
0.0 & 0 \\
0.0 & 0 \\
0.0 & 0 \\
0.0 & 0 \\
0.0 & 0\end{array}$ \\
\hline
\end{tabular}


Annual

27 Deorees North Lotitude

$\begin{array}{llllll}\text { WPT } & 126 & 122 & 118 & 114 & 110 \\ & & & & & \\ 1 & 0.0 & 0.0 & 0.0 & 0.0 & 0.0 \\ ? & 0.0 & 0.0 & 0.0 & 0.0 & 0.0 \\ 3 & 0.0 & 0.0 & 0.0 & 0.0 & 0.0 \\ 4 & 0.0 & 0.0 & 0.0 & 0.0 & 0.0 \\ 5 & 0.0 & 0.0 & 0.0 & 0.0 & 0.0 \\ 6 & 0.0 & 0.0 & 0.0 & 0.0 & 0.0 \\ 7 & 0.0 & 0.0 & 0.0 & 0.0 & 0.0 \\ 8 & 0.0 & 0.0 & 0.0 & 0.0 & 0.0 \\ 9 & 0.0 & 0.0 & 0.0 & 0.0 & 0.0\end{array}$

Degress west Lonot tutde

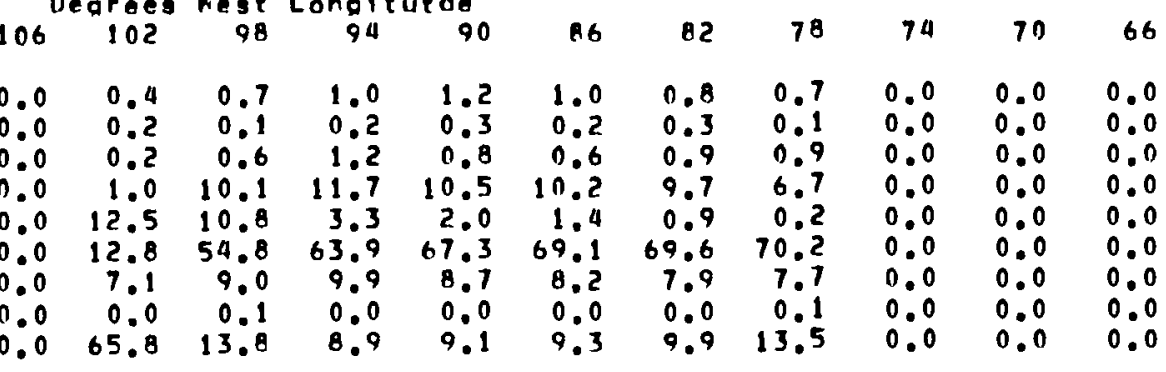

24 Degreos North Latitude

$\begin{array}{lll}0 & 13.7 & 15.7\end{array}$ 
Hinter

51 Dearees North Latitude

\begin{tabular}{|c|c|c|c|c|c|c|c|c|c|c|c|c|c|c|c|c|}
\hline MPT & 126 & 122 & 118 & 114 & 110 & $106^{\circ}$ & $\begin{array}{l}\text { earees } \\
102\end{array}$ & $\begin{array}{r}\text { Wegt } \\
98\end{array}$ & $\begin{array}{c}\text { Longle } \\
94\end{array}$ & $\begin{array}{l}\text { de } \\
90\end{array}$ & 86 & 82 & 78 & 74 & 70 & 66 \\
\hline $\begin{array}{l}1 \\
2 \\
3 \\
4 \\
5 \\
6 \\
7 \\
8 \\
9\end{array}$ & $\begin{array}{r}1.9 \\
25.6 \\
1.0 \\
7.0 \\
22.6 \\
0.0 \\
10.8 \\
10.0 \\
20.7\end{array}$ & $\begin{array}{r}1.3 \\
18.4 \\
0.9 \\
12.9 \\
32.3 \\
0.0 \\
15.2 \\
6.9 \\
12.2\end{array}$ & $\begin{array}{r}1.9 \\
10.5 \\
1.0 \\
34.3 \\
15.2 \\
0.0 \\
22.5 \\
6.3 \\
8.3\end{array}$ & $\begin{array}{r}3.2 \\
6.3 \\
1.2 \\
24.2 \\
17.6 \\
0.0 \\
28.7 \\
6.4 \\
10.3\end{array}$ & $\begin{array}{r}3.3 \\
6.4 \\
1.7 \\
20.4 \\
24.3 \\
0.0 \\
22.3 \\
7.3 \\
12.0\end{array}$ & $\begin{array}{r}3.0 \\
12.0 \\
1.7 \\
22.9 \\
25.7 \\
0.0 \\
16.9 \\
7.4 \\
10.4\end{array}$ & $\begin{array}{r}3.2 \\
10.5 \\
2.4 \\
25.6 \\
26.9 \\
0.0 \\
13.5 \\
9.1 \\
8.6\end{array}$ & $\begin{array}{r}3.0 \\
12.5 \\
1.7 \\
33.3 \\
23.2 \\
0.0 \\
8.5 \\
10.6 \\
8.2\end{array}$ & $\begin{array}{r}1.8 \\
14.7 \\
2.1 \\
34.9 \\
19.6 \\
0.0 \\
6.1 \\
12.4 \\
8.3\end{array}$ & $\begin{array}{l}0.0 \\
0.0 \\
0.0 \\
0.0 \\
0.0 \\
0.0 \\
0.0 \\
0.0 \\
0.0\end{array}$ & $\begin{array}{l}0.0 \\
0.0 \\
0.0 \\
0.0 \\
0.0 \\
0.0 \\
0.0 \\
0.0 \\
0.0\end{array}$ & $\begin{array}{l}0.0 \\
0.0 \\
0.0 \\
0.0 \\
0.0 \\
0.0 \\
0.0 \\
0.0 \\
0.0 \\
0.0\end{array}$ & $\begin{array}{ll}0 & 0 \\
0 & 0 \\
0 & 0 \\
0 & 0 \\
0 & 0 \\
0 & 0 \\
0 & 0 \\
0 & 0 \\
0 & 0 \\
0 & 0 \\
0.0 & 0\end{array}$ & $\begin{array}{l}0.0 \\
0.0 \\
0.0 \\
0.0 \\
0.0 \\
0.0 \\
0.0 \\
0.0 \\
0.0\end{array}$ & 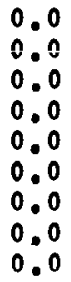 & $\begin{array}{l}0.0 \\
0.0 \\
0.0 \\
0.0 \\
0.0 \\
0.0 \\
0.0 \\
0.0 \\
0.0\end{array}$ \\
\hline
\end{tabular}

48 Degrees North Lettidude

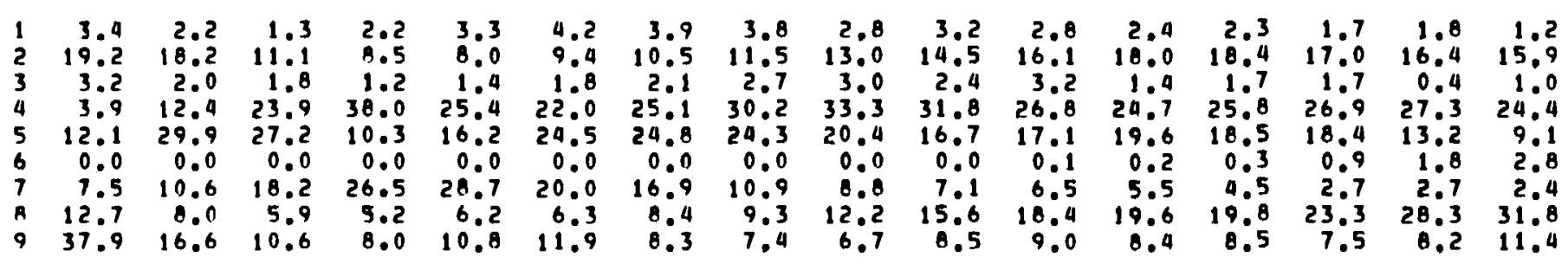

45 Deorees North Lettede

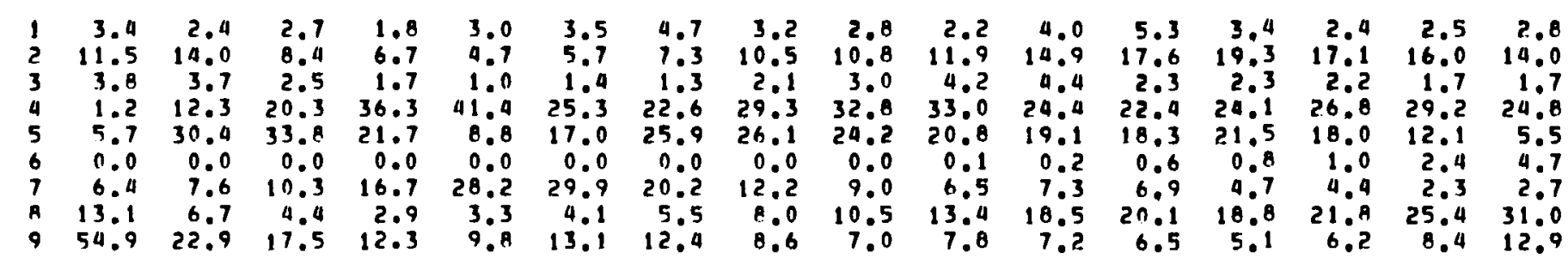

42 Degrees North Lotitudo

\begin{tabular}{|c|c|c|c|c|c|c|c|c|c|c|c|c|c|c|c|}
\hline $\begin{array}{r}3.3 \\
7.5 \\
5.0 \\
0.1 \\
2.1 \\
0.0 \\
6.5 \\
9.0 \\
67.4\end{array}$ & $\begin{array}{r}2.7 \\
7.9 \\
3.0 \\
11.8 \\
33.5 \\
0.0 \\
7.3 \\
5.2 \\
28.7\end{array}$ & $\begin{array}{r}2.1 \\
5.5 \\
3.2 \\
19.0 \\
40.1 \\
0.0 \\
6.0 \\
3.7 \\
20.0\end{array}$ & $\begin{array}{r}1.7 \\
6.0 \\
2.0 \\
27.7 \\
34.0 \\
0.0 \\
10.0 \\
2.7 \\
15.6\end{array}$ & $\begin{array}{r}2.1 \\
5.3 \\
1.7 \\
49.4 \\
12.5 \\
0.0 \\
15.2 \\
1.8 \\
12.0\end{array}$ & $\begin{array}{r}2.5 \\
4.9 \\
1.9 \\
35.1 \\
8.5 \\
0.0 \\
28.9 \\
2.9 \\
15.4\end{array}$ & $\begin{array}{r}2.9 \\
5.7 \\
2.0 \\
20.5 \\
25.8 \\
0.0 \\
21.4 \\
2.9 \\
18.8\end{array}$ & $\begin{array}{r}2 . A \\
7.0 \\
2.0 \\
26.7 \\
29.9 \\
0.1 \\
13.4 \\
5.1 \\
13.0\end{array}$ & $\begin{array}{r}3.3 \\
8.5 \\
2.8 \\
32.5 \\
28.3 \\
0.2 \\
9.8 \\
7.2 \\
7.4\end{array}$ & $\begin{array}{r}3.4 \\
10.3 \\
4.7 \\
33.1 \\
25.6 \\
0.2 \\
7.2 \\
10.1 \\
5.3\end{array}$ & $\begin{array}{r}4.0 \\
12.2 \\
4.5 \\
27.8 \\
24.1 \\
0.7 \\
7.1 \\
15.0 \\
4.7\end{array}$ & $\begin{array}{r}6.0 \\
13.6 \\
2.9 \\
20.3 \\
23.3 \\
1.0 \\
6.3 \\
17.3\end{array}$ & $\begin{array}{r}3.8 \\
15.6 \\
3.7 \\
25.6 \\
22.3 \\
1.2 \\
5.7 \\
17.1\end{array}$ & $\begin{array}{r}2.8 \\
14.9 \\
4.0 \\
32.3 \\
15.7 \\
2.4 \\
3.9 \\
16.6\end{array}$ & $\begin{array}{r}3.8 \\
13.0 \\
4.1 \\
30.5 \\
7.1 \\
4.4 \\
3.8 \\
22.4 \\
11.0\end{array}$ & 27 \\
\hline
\end{tabular}


ninter

39 Deqrees North Lot ltude

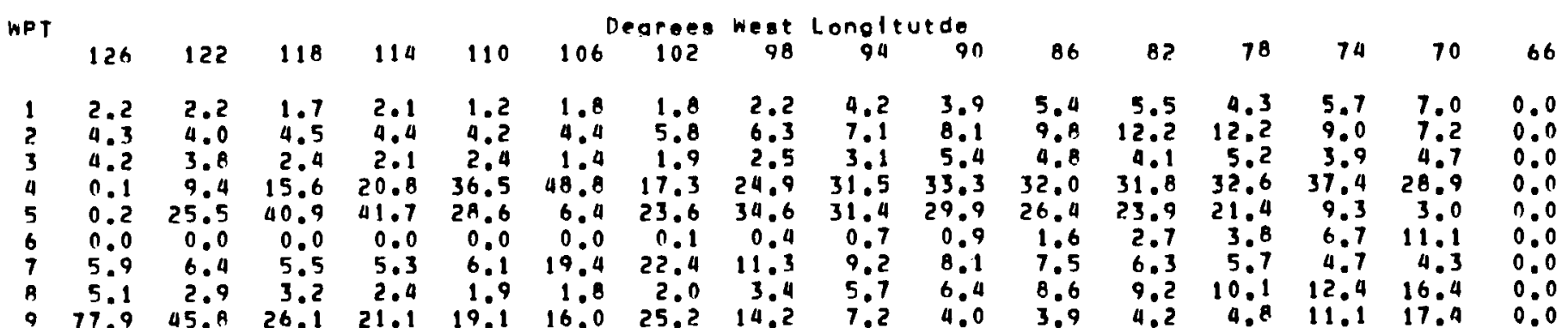

36 Deareea North Latitude

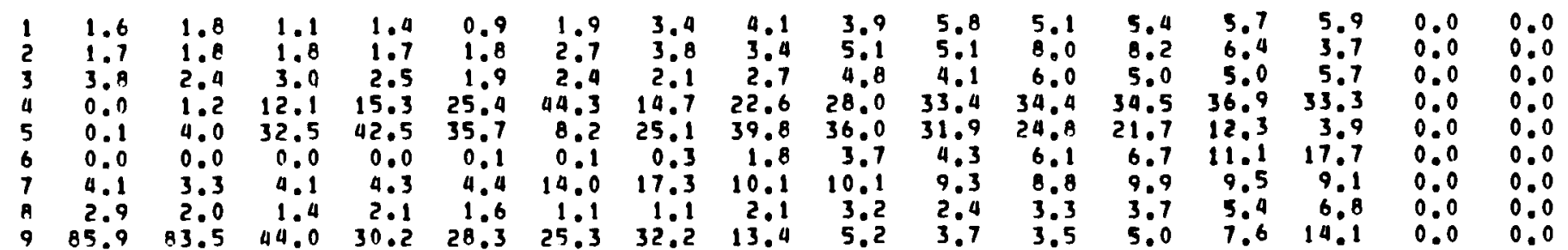

33 Dearees North Latitude

\begin{tabular}{|c|c|c|c|c|c|c|c|c|c|c|c|c|c|c|}
\hline $\begin{array}{l}0.0 \\
0.0 \\
0.0 \\
0.0 \\
0.0 \\
0.0 \\
0.00 \\
0.0 \\
0.0\end{array}$ & $\begin{array}{r}1.3 \\
0.6 \\
2.0 \\
0.0 \\
0.2 \\
0.0 \\
2.2 \\
0.9 \\
92.8\end{array}$ & $\begin{array}{r}0.6 \\
0.8 \\
2.2 \\
5.9 \\
8.9 \\
0.0 \\
3.8 \\
0.7 \\
77.3\end{array}$ & $\begin{array}{r}0.8 \\
0.4 \\
2.3 \\
10.1 \\
23.7 \\
0.0 \\
3.8 \\
0.8 \\
58.1\end{array}$ & $\begin{array}{r}1.3 \\
0.8 \\
1.6 \\
15.9 \\
34.0 \\
0.0 \\
4.1 \\
1.2 \\
41.1\end{array}$ & $\begin{array}{r}2.2 \\
1.1 \\
1.1 \\
30.0 \\
11.0 \\
0.1 \\
11.0 \\
0.8 \\
42.1\end{array}$ & $\begin{array}{r}2.9 \\
2.1 \\
2.3 \\
11.5 \\
30.5 \\
2.0 \\
12.1 \\
0.6 \\
36.0\end{array}$ & $\begin{array}{r}3.1 \\
2.7 \\
2.5 \\
20.2 \\
38.7 \\
8.0 \\
9.5 \\
1.2 \\
14.1\end{array}$ & $\begin{array}{r}4.4 \\
2.5 \\
3.9 \\
27.4 \\
31.3 \\
12.9 \\
10.6 \\
1.7 \\
5.3\end{array}$ & $\begin{array}{r}4.8 \\
4.0 \\
2.9 \\
33.3 \\
25.8 \\
10.3 \\
9.2 \\
1.9 \\
3.9\end{array}$ & $\begin{array}{r}4.3 \\
5.7 \\
4.2 \\
35.0 \\
21.2 \\
15.5 \\
9.1 \\
1.0 \\
4.0\end{array}$ & $\begin{array}{r}4.7 \\
5.0 \\
4.8 \\
35.8 \\
14.9 \\
10.4 \\
10.4 \\
0.9 \\
5.2\end{array}$ & $\begin{array}{r}4.5 \\
2.3 \\
5.3 \\
35.5 \\
5.3 \\
25.4 \\
10.2 \\
1.6 \\
9.9\end{array}$ & $\begin{array}{r}5 . \\
1 . \\
50 \\
26 \\
0 . \\
30 \\
9 . \\
3 . \\
17 .\end{array}$ & $\begin{array}{l}0.0 \\
0.0 \\
0.0 \\
0.0 \\
0.0 \\
0.0 \\
0.0 \\
0.00 \\
0.0\end{array}$ \\
\hline
\end{tabular}

30 Dearees North Lotitude

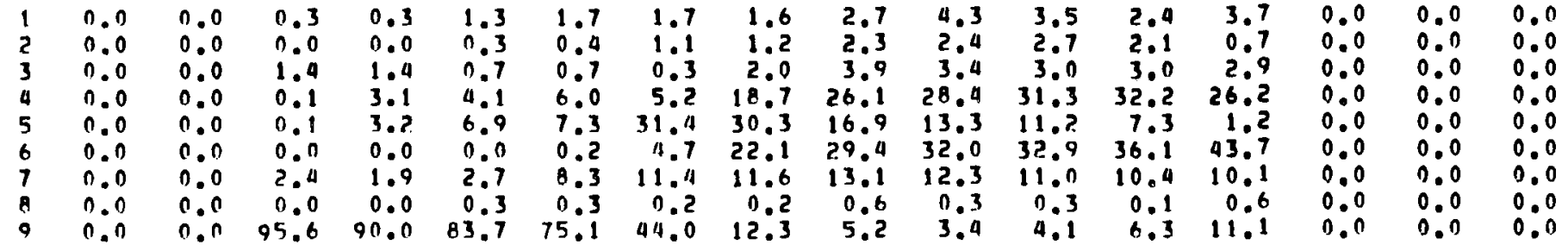


ninter

27 Deorees North Latleude

\begin{tabular}{|c|c|c|c|c|c|c|c|c|c|c|c|c|c|c|c|c|}
\hline MPT & 126 & 122 & 118 & 114 & 110 & 106 & $\begin{array}{c}\text { ogrees } \\
102\end{array}$ & $\begin{array}{r}\text { Weat } \\
98\end{array}$ & $\begin{array}{c}\text { Longlt } \\
94\end{array}$ & toe & 86 & 82 & $7^{B}$ & 74 & 70 & 66 \\
\hline $\begin{array}{l}1 \\
2 \\
3 \\
4 \\
5 \\
6 \\
7 \\
8 \\
9\end{array}$ & $\begin{array}{l}0.0 \\
0.0 \\
0.0 \\
0.0 \\
0.0 \\
0.0 \\
0.0 \\
0.0 \\
0.0\end{array}$ & $\begin{array}{l}0.0 \\
0.00 \\
0.0 \\
0.0 \\
0.0 \\
0.0 \\
0.0 \\
0.0 \\
0.0 \\
0.0\end{array}$ & $\begin{array}{l}0.0 \\
0.00 \\
0.0 \\
0.0 \\
0.0 \\
0.0 \\
0.00 \\
0.0 \\
0.0\end{array}$ & $\begin{array}{l}0.0 \\
0.0 \\
0.0 \\
0.0 \\
0.0 \\
0.0 \\
0.0 \\
0.0 \\
0.0 \\
0.0\end{array}$ & $\begin{array}{l}0.0 \\
0.0 \\
0.0 \\
0.0 \\
0.0 \\
0.0 \\
0.0 \\
0.0 \\
0.0\end{array}$ & $\begin{array}{lll}0 & 0 & 0 \\
0 & 0 & 0 \\
0 & 0 & 0 \\
0 . & 0 \\
0.0 & 0 \\
0.0 & 0 \\
0 & 0 & 0 \\
0.0 & 0 \\
0.0 & 0\end{array}$ & $\begin{array}{r}0.4 \\
0.2 \\
0.4 \\
1.6 \\
19.0 \\
7.8 \\
10.9 \\
0.0 \\
59.8\end{array}$ & $\begin{array}{r}1.1 \\
0.1 \\
1.4 \\
17.2 \\
18.4 \\
32.0 \\
13.9 \\
0.2 \\
15.6\end{array}$ & $\begin{array}{r}1.9 \\
0.3 \\
3.3 \\
21.0 \\
7.0 \\
42.8 \\
15.0 \\
0.0 \\
8.8\end{array}$ & $\begin{array}{r}3.0 \\
0.6 \\
1.4 \\
20.4 \\
5.1 \\
48.8 \\
13.3 \\
0.1 \\
7.3\end{array}$ & $\begin{array}{r}2.3 \\
0.3 \\
1.2 \\
20.4 \\
3.1 \\
52.5 \\
13.5 \\
0.0 \\
6.5\end{array}$ & $\begin{array}{r}1.3 \\
0.7 \\
1.8 \\
19.8 \\
2.2 \\
54.0 \\
12.6 \\
0.0 \\
7.5\end{array}$ & $\begin{array}{r}1.9 \\
0.2 \\
1.9 \\
19.6 \\
0.4 \\
57.9 \\
11.6 \\
0.0 \\
11.4\end{array}$ & $\begin{array}{l}0.0 \\
0.0 \\
0.0 \\
0.0 \\
0.0 \\
0.0 \\
0.0 \\
0.0 \\
0.00 \\
0.0\end{array}$ & $\begin{array}{l}0.0 \\
0.0 \\
0.0 \\
0.0 \\
0.0 \\
0.0 \\
0.0 \\
0.0 \\
0.0\end{array}$ & $\begin{array}{l}0.0 \\
0.0 \\
0.0 \\
0.0 \\
0.0 \\
0.0 \\
0.0 \\
0.0 \\
0.0\end{array}$ \\
\hline
\end{tabular}

24 Degraes North Latitude

$\begin{array}{llllll}1 & 0.0 & 0.0 & 0.0 & 0.0 & 0.0 \\ 2 & 0.0 & 0.0 & 0.0 & 0.0 & 0.0 \\ 3 & 0.0 & 0.0 & 0.0 & 0.0 & 0.0 \\ 4 & 0.0 & 0.0 & 0.0 & 0.0 & 0.0 \\ 5 & 0.0 & 0.0 & 0.0 & 0.0 & 0.0 \\ 6 & 0.0 & 0.0 & 0.0 & 0.0 & 0.0 \\ 7 & 0.0 & 0.0 & 0.0 & 0.0 & 0.0 \\ 9 & 0.0 & 0.0 & 0.0 & 0.0 & 0.0 \\ 9 & 0.0 & 0.0 & 0.0 & 0.0 & 0.0\end{array}$

$\begin{array}{rrrrrrrrrrr}0.0 & 0.0 & 0.3 & 0.0 & 0.0 & 0.0 & 0.6 & 0.4 & 0.0 & 0.0 & 0.0 \\ 0.0 & 0.0 & 0.0 & 0.0 & 0.0 & 0.0 & 0.0 & 0.0 & 0.0 & 0.0 & 0.0 \\ 0.0 & 0.0 & 0.8 & 0.0 & 0.0 & 0.0 & 0.9 & 0.0 & 0.0 & 0.0 & 0.0 \\ 0.0 & 0.0 & 12.7 & 0.0 & 0.0 & 0.0 & 0.0 & 5.7 & 0.0 & 0.0 & 0.0 \\ 0.0 & 0.0 & 11.9 & 0.0 & 0.0 & 0.0 & 0.3 & 0.0 & 0.0 & 0.0 & 0.0 \\ 0.0 & 0.0 & 37.5 & 0.0 & 0.0 & 0.0 & 66.1 & 67.3 & 0.0 & 0.0 & 0.0 \\ 0.0 & 0.0 & 10.9 & 0.0 & 0.0 & 0.0 & 14.2 & 12.1 & 0.0 & 0.0 & 0.0 \\ 0.0 & 0.0 & 0.0 & 0.0 & 0.0 & 0.0 & 0.0 & 0.0 & 0.0 & 0.0 & 0.0 \\ 0.0 & 0.0 & 25.8 & 0.0 & 0.0 & 0.0 & 9.2 & 19.1 & 0.0 & 0.0 & 0.0\end{array}$


Sorina

51 Dearees North Lotitude

\begin{tabular}{|c|c|c|c|c|c|c|c|c|c|c|c|c|c|c|c|c|}
\hline WPT & 126 & 122 & 118 & 114 & 110 & $106^{\circ}$ & $\begin{array}{c}\text { eareas } \\
102\end{array}$ & $\begin{array}{r}\text { Weat } \\
9 B\end{array}$ & $\begin{array}{c}\text { Lonoit } \\
94\end{array}$ & 90 & 86 & 82 & 78 & 74 & 70 & 66 \\
\hline $\begin{array}{l}1 \\
2 \\
3 \\
4 \\
5 \\
6 \\
7 \\
8 \\
9\end{array}$ & $\begin{array}{r}0.7 \\
16.0 \\
1.1 \\
20.1 \\
0.9 \\
0.0 \\
5.0 \\
9.7\end{array}$ & $\begin{array}{r}0.5 \\
10.3 \\
0.8 \\
27.0 \\
15.7 \\
0.0 \\
6.7 \\
7.4 \\
30.7\end{array}$ & $\begin{array}{r}0.9 \\
6.8 \\
1.6 \\
31.3 \\
14.9 \\
0.0 \\
12.5 \\
7.9 \\
24.0\end{array}$ & $\begin{array}{r}1.9 \\
6.7 \\
2.3 \\
25.2 \\
17.3 \\
0.0 \\
17.7 \\
8.9\end{array}$ & $\begin{array}{r}2.0 \\
9.6 \\
2.1 \\
19.8 \\
25.3 \\
0.0 \\
13.8 \\
9.0 \\
18.5\end{array}$ & $\begin{array}{r}2.0 \\
11.6 \\
2.1 \\
16.7 \\
31.8 \\
0.0 \\
12.3 \\
9.6 \\
13.0\end{array}$ & $\begin{array}{r}2.5 \\
10.8 \\
2.1 \\
19.6 \\
35.0 \\
0.0 \\
9.0 \\
9.9\end{array}$ & $\begin{array}{r}2.8 \\
11.4 \\
2.8 \\
23.0 \\
34.2 \\
0.0 \\
7.1 \\
9.9 \\
8.7\end{array}$ & $\begin{array}{r}3.3 \\
13.5 \\
2.7 \\
24.7 \\
33.7 \\
0.0 \\
6.6 \\
9.7 \\
7.4\end{array}$ & $\begin{array}{ll}0.0 & 0 \\
0.0 & 0 \\
0.0 & 0 \\
0.0 & 0 \\
0.0 & 0 \\
0.0 & 0 \\
0.0 & 0 \\
0.0 & 0 \\
0.0 & 0\end{array}$ & $\begin{array}{l}0.0 \\
0.0 \\
0.0 \\
0.0 \\
0.0 \\
0.0 \\
0.0 \\
0.0 \\
0.0\end{array}$ & $\begin{array}{ll}0.0 & 0 \\
0.0 & 0 \\
0.0 & 0 \\
0.0 & 0 \\
0.0 & 0 \\
0.0 & 0 \\
0.0 & 0 \\
0.0 & 0 \\
0.0 & 0\end{array}$ & $\begin{array}{l}0.0 \\
0.0 \\
0.0 \\
0.0 \\
0.0 \\
0.0 \\
0.0 \\
0.0 \\
0.0\end{array}$ & $\begin{array}{l}0.0 \\
0.0 \\
0.0 \\
0.0 \\
0.0 \\
0.0 \\
0.0 \\
0.0 \\
0.0\end{array}$ & 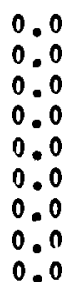 & $\begin{array}{ll}0 & 0 \\
0 & 0 \\
0 & 0 \\
0.0 & 0 \\
0.0 & 0 \\
0.0 & 0 \\
0.0 & 0 \\
0.0 & 0 \\
0.0 & 0\end{array}$ \\
\hline
\end{tabular}

$4 B$ Degrees North Lotitude

\begin{tabular}{|c|c|c|c|c|c|c|c|c|c|c|c|c|c|c|c|}
\hline $\begin{array}{rr}1 & 1.2 \\
2 & 11.4 \\
3 & 2.1 \\
4 & 11.8 \\
5 & 4.1 \\
6 & 0.0 \\
7 & 3.8 \\
8 & 6.6 \\
9 & 57.0\end{array}$ & $\begin{array}{r}0.9 \\
10.0 \\
1.1 \\
29.7 \\
12.8 \\
0.0 \\
4.7 \\
7.1 \\
33.8\end{array}$ & $\begin{array}{r}0.7 \\
7.6 \\
1.3 \\
30.0 \\
17.6 \\
0.0 \\
7.7 \\
7.3 \\
27.8\end{array}$ & $\begin{array}{r}1.3 \\
7.0 \\
3.0 \\
31.5 \\
17.1 \\
0.0 \\
12.6 \\
8.0 \\
19.5\end{array}$ & $\begin{array}{r}2.9 \\
7.9 \\
3.3 \\
23.7 \\
19.6 \\
0.0 \\
15.8 \\
8.6 \\
18.4\end{array}$ & $\begin{array}{r}3.5 \\
9.6 \\
3.2 \\
22.0 \\
25.3 \\
0.0 \\
12.7 \\
8.0 \\
15.8\end{array}$ & $\begin{array}{r}3.7 \\
12.5 \\
2.7 \\
20.7 \\
29.8 \\
0.0 \\
10.9 \\
8.5 \\
11.3\end{array}$ & $\begin{array}{r}3.3 \\
12.3 \\
2.8 \\
23.5 \\
30.4 \\
0.0 \\
8.3 \\
10.8 \\
8.7\end{array}$ & $\begin{array}{r}3.4 \\
12.8 \\
2.7 \\
24.2 \\
32.2 \\
0.1 \\
7.0 \\
11.3\end{array}$ & $\begin{array}{r}3.5 \\
12.9 \\
2.3 \\
27.2 \\
31.0 \\
0.1 \\
5.0 \\
12.5 \\
5.5\end{array}$ & $\begin{array}{r}2.9 \\
12.9 \\
2.2 \\
30.7 \\
26.0 \\
0.1 \\
4.9 \\
19.0 \\
6.3\end{array}$ & $\begin{array}{r}2.9 \\
13.3 \\
2.3 \\
32.2 \\
22.0 \\
0.3 \\
0.2 \\
16.0 \\
6.9\end{array}$ & $\begin{array}{r}2.8 \\
13.8 \\
2.1 \\
31.0 \\
19.2 \\
0.3 \\
5.3 \\
18.3\end{array}$ & $\begin{array}{r}2 . \\
14 . \\
2 . \\
30 \\
16 . \\
0 . \\
40 \\
20\end{array}$ & $\begin{array}{r}2.3 \\
14.7 \\
1.4 \\
26.0 \\
12.5\end{array}$ & $\begin{array}{r}2 \\
15\end{array}$ \\
\hline
\end{tabular}

45 Deareee North l.etieude

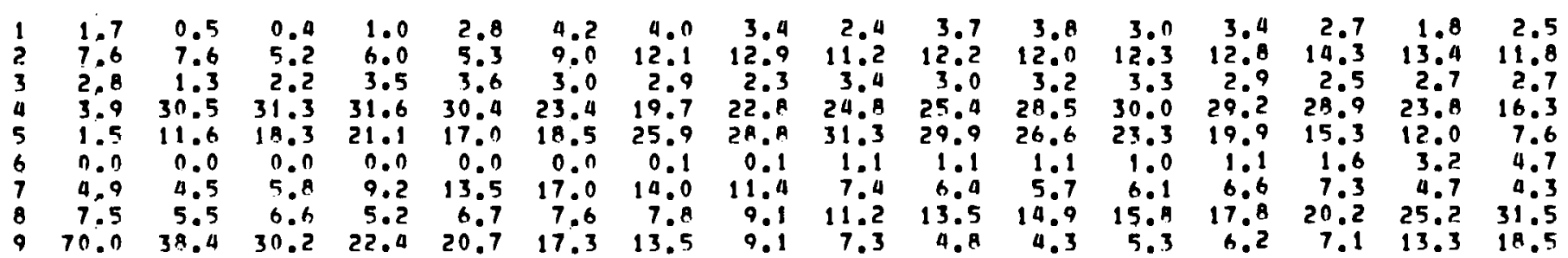

42 Dearees North l.etitude

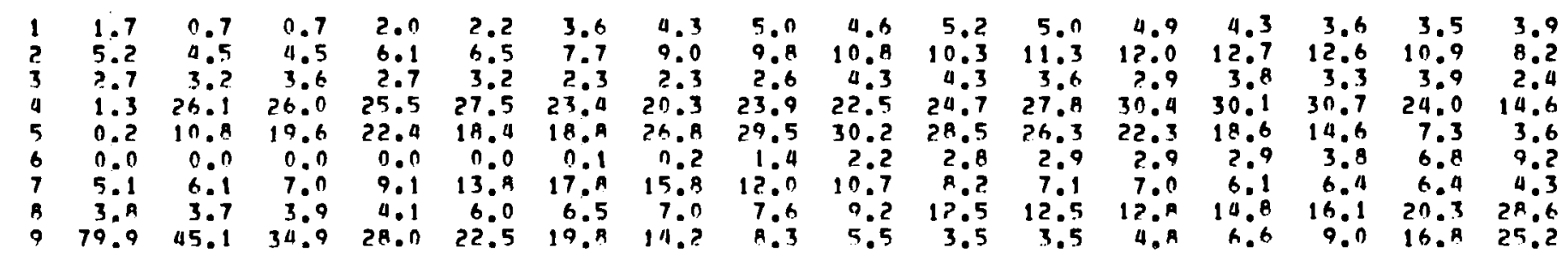


Sprino

39 Degrees North Lotitude

\begin{tabular}{|c|c|c|c|c|c|c|c|c|c|c|c|c|c|c|c|c|}
\hline WPT & 126 & 122 & 118 & 114 & 110 & $106^{\circ}$ & $\begin{array}{c}\text { araes } \\
102\end{array}$ & $\begin{array}{r}\text { West } \\
98\end{array}$ & $\begin{array}{c}\text { Lompltt } \\
94\end{array}$ & $\begin{array}{l}\text { utde } \\
90\end{array}$ & 86 & 82 & $7^{A}$ & 74 & 70 & 66 \\
\hline $\begin{array}{l}1 \\
? \\
3 \\
4 \\
5 \\
6 \\
7 \\
6 \\
9\end{array}$ & $\begin{array}{r}1.3 \\
1.6 \\
2.8 \\
0.5 \\
0.1 \\
0.0 \\
5.0 \\
2.8 \\
85.8\end{array}$ & $\begin{array}{r}0.7 \\
2.9 \\
3.6 \\
16.6 \\
5.0 \\
0.0 \\
6.6 \\
2.0 \\
62.6\end{array}$ & $\begin{array}{r}0.3 \\
3.3 \\
3.3 \\
19.8 \\
17.9 \\
0.0 \\
6.6 \\
3.3 \\
45.5\end{array}$ & $\begin{array}{r}2.2 \\
4.7 \\
3.4 \\
18.0 \\
22.6 \\
0.0 \\
9.0 \\
3.5 \\
36.6\end{array}$ & $\begin{array}{r}3.5 \\
4.7 \\
2.6 \\
20.4 \\
22.4 \\
0.0 \\
10.3 \\
4.2 \\
31.8\end{array}$ & $\begin{array}{r}3.4 \\
5.5 \\
2.6 \\
21.4 \\
19.2 \\
0.1 \\
17.1 \\
4.8 \\
25.4\end{array}$ & $\begin{array}{r}4.5 \\
6.3 \\
3.4 \\
18.8 \\
26.1 \\
0.7 \\
17.4 \\
4.5 \\
18.5\end{array}$ & $\begin{array}{r}5.0 \\
7.7 \\
4.3 \\
21.5 \\
27.9 \\
3.7 \\
15.1 \\
5.8 \\
8.9\end{array}$ & $\begin{array}{r}5.9 \\
3.7 \\
4.9 \\
21.3 \\
29.7 \\
6.9 \\
11.7 \\
7.2 \\
4.2\end{array}$ & $\begin{array}{r}5.0 \\
8.3 \\
5.1 \\
24.0 \\
28.5 \\
8.4 \\
9.6 \\
8.2 \\
3.0\end{array}$ & $\begin{array}{r}5.8 \\
8.4 \\
4.5 \\
28.5 \\
29.7 \\
7.5 \\
9.9 \\
7.7 \\
3.2\end{array}$ & $\begin{array}{r}7.0 \\
9.1 \\
4.2 \\
31.5 \\
21.1 \\
6.3 \\
9.1 \\
7.0 \\
4.7\end{array}$ & $\begin{array}{r}6.0 \\
10.7 \\
4.7 \\
30.5 \\
17.4 \\
7.0 \\
8.3 \\
7.5 \\
9.0\end{array}$ & $\begin{array}{r}5.7 \\
8.9 \\
4.5 \\
31.2 \\
8.5 \\
10.8 \\
7.7 \\
10.4 \\
12.4\end{array}$ & $\begin{array}{r}5.2 \\
6.6 \\
4.0 \\
20.5 \\
3.4 \\
14.2 \\
6.0 \\
16.4 \\
23.6\end{array}$ & 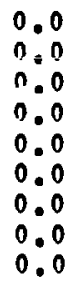 \\
\hline
\end{tabular}

36 Dearees North Lotitude

$\begin{array}{rrrrrrrrrrr}2.8 & 4.0 & 5.8 & 5.9 & 5.8 & 5.8 & 5.3 & 6.0 & 6.5 & 0.0 & 0.0 \\ 2.6 & 3.9 & 4.5 & 5.5 & 4.7 & 6.2 & 6.7 & 5.9 & 3.8 & 0.0 & 0.0 \\ 3.7 & 5.0 & 3.7 & 4.2 & 3.9 & 4.1 & 4.9 & 4.3 & 5.0 & 0.0 & 0.0 \\ 18.9 & 15.1 & 19.8 & 20.9 & 24.7 & 28.4 & 29.1 & 30.3 & 23.3 & 0.0 & 0.0 \\ 19.6 & 26.8 & 30.5 & 29.3 & 26.3 & 23.3 & 19.0 & 12.2 & 3.7 & 0.0 & 0.0 \\ 0.2 & 2.6 & 11.4 & 15.1 & 18.6 & 17.7 & 16.1 & 17.3 & 21.6 & 0.0 & 0.0 \\ 12.4 & 15.0 & 12.6 & 11.3 & 9.3 & 8.3 & 9.7 & 10.7 & 11.1 & 0.0 & 0.0 \\ 3.5 & 2.9 & 2.6 & 3.7 & 4.0 & 3.3 & 3.9 & 4.1 & 6.4 & 0.0 & 0.0 \\ 36.3 & 24.6 & 9.1 & 4.0 & 2.7 & 3.0 & 5.2 & 9.2 & 18.6 & 0.0 & 0.0\end{array}$

33 Deareas North Latitude

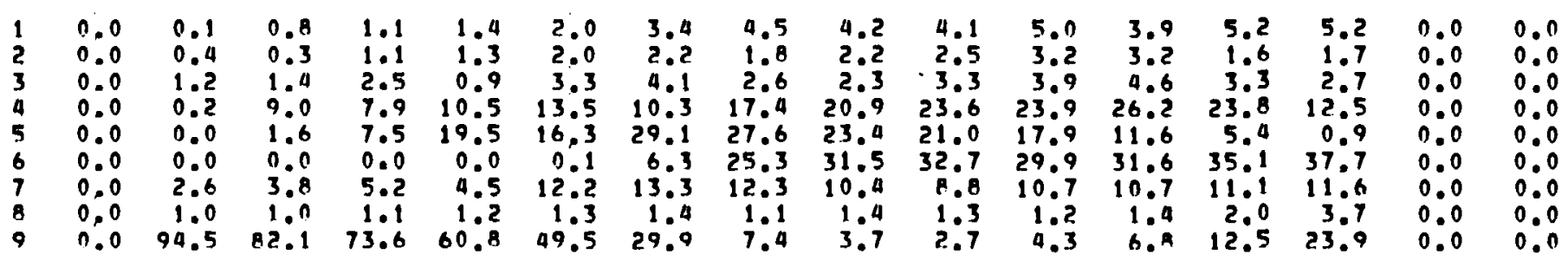

3 Dearees North Latitude

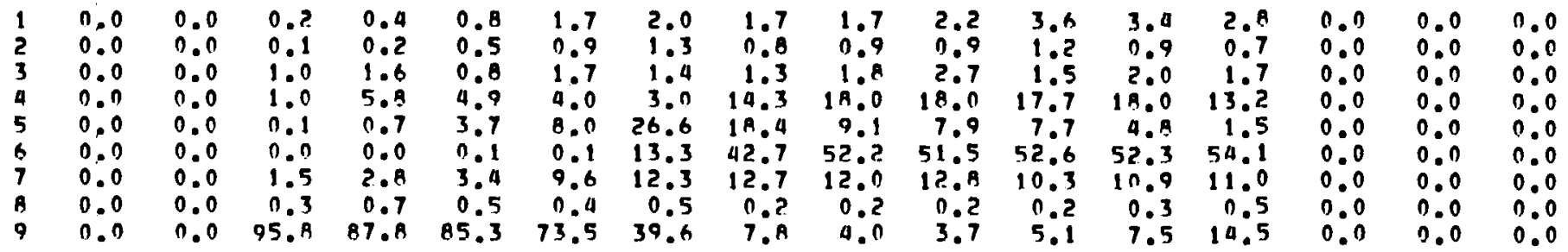


Spring

27 Dearees North Latitude

WPT

$\begin{array}{lllll}126 & 122 \quad 118 & 114 & 110\end{array}$

0

$$
\begin{aligned}
& 0 \\
& 0 \\
& 0
\end{aligned}
$$

$\begin{array}{llll}0.0 & 0.0 & 0.0 & 0.0 \\ 0.0 & 0.0 & 0.0 & 0.0\end{array}$

$\begin{array}{llll}0.0 & 0.0 & 0.0 & 0.0 \\ 0.0 & 0.0 & 0.0 & 0.0\end{array}$

$\begin{array}{llll}0.0 & 0.0 & 0.0 & 0.0 \\ 0.0 & 0.0 & 0.0 & 0.0 \\ 0.0 & 0.0 & 0.0 & 0.0\end{array}$

$\begin{array}{lllll}6.0 & 0.0 & 0.0 & 0.0\end{array}$

$\begin{array}{llllll}7 & 0.0 & 0.0 & 0.0 & 0.0 & 0.0 \\ 0 & 0.0 & 0.0 & 0.0 & 0.0 & 0.0 \\ 9 & 0.0 & 0.0 & 0.0 & 0.0 & 0.0\end{array}$

Dearnes Weat Lonal tutde

$\begin{array}{rrrrrrrrrrr}106 & 102 & 98 & 94 & 90 & 86 & 82 & 78 & 74 & 70 & 66 \\ 0.0 & 0.9 & 1.0 & 1.5 & 1.0 & 1.0 & 1.3 & 0.4 & 0.0 & 0.0 & 0.0 \\ 0.0 & 0.3 & 0.2 & 0.1 & 0.2 & 0.2 & 0.2 & 0.2 & 0.0 & 0.0 & 0.0 \\ 0.0 & 0.5 & 0.3 & 0.9 & 1.0 & 0.5 & 1.0 & 1.1 & 0.0 & 0.0 & 0.0 \\ 0.0 & 0.2 & 9.6 & 9.9 & 8.9 & 8.9 & 8.7 & 5.5 & 0.0 & 0.0 & 0.0 \\ 0.0 & 12.9 & 10.4 & 2.5 & 1.5 & 1.7 & 1.4 & 0.3 & 0.0 & 0.0 & 0.0 \\ 0.0 & 13.3 & 55.5 & 67.8 & 70.7 & 71.1 & 70.7 & 70.4 & 0.0 & 0.0 & 0.0 \\ 0.0 & 9.7 & 11.4 & 11.5 & 9.9 & 8.3 & 7.8 & 0.4 & 0.0 & 0.0 & 0.0 \\ 0.0 & 0.0 & 0.0 & 0.1 & 0.0 & 0.0 & 0.1 & 0.1 & 0.0 & 0.0 & 0.0 \\ 0.0 & 62.2 & 11.5 & 5.7 & 6.4 & 7.0 & 8.8 & 13.5 & 0.0 & 0.0 & 0.0\end{array}$

24 Dearees North Latitude

$\begin{array}{lllllll}1 & 0.0 & 0.0 & 0.0 & 0.0 & 0.0 & 0.0 \\ 2 & 0.0 & 0.0 & 0.0 & 0.0 & 0.0 & 0.0 \\ 3 & 0.0 & 0.0 & 0.0 & 0.0 & 0.0 & 0.0 \\ 4 & 0.0 & 0.0 & 0.0 & 0.0 & 0.0 & 0.0 \\ 5 & 0.0 & 0.0 & 0.0 & 0.0 & 0.0 & 0.0 \\ 6 & 0.0 & 0.0 & 0.0 & 0.0 & 0.0 & 0.0 \\ 7 & 0.0 & 0.0 & 0.0 & 0.0 & 0.0 & 0.0 \\ 8 & 0.0 & 0.0 & 0.0 & 0.0 & 0.0 & 0.0 \\ 9 & 0.0 & 0.0 & 0.0 & 0.0 & 0.0 & 0.0\end{array}$

$\begin{array}{rrrrrrrrrrr}0.0 & 0.0 & 0.3 & 0.0 & 0.0 & 0.0 & 0.3 & 0.4 & 0.0 & 0.0 & 0.0 \\ 0.0 & 0.0 & 0.1 & 0.0 & 0.0 & 0.0 & 0.1 & 0.0 & 0.0 & 0.0 & 0.0 \\ 0.0 & 0.0 & 0.1 & 0.0 & 0.0 & 0.0 & 0.2 & 0.1 & 0.0 & 0.0 & 0.0 \\ 0.0 & 0.0 & 4.8 & 0.0 & 0.0 & 0.0 & 2.3 & 1.1 & 0.0 & 0.0 & 0.0 \\ 0.0 & 0.0 & 5.8 & 0.0 & 0.0 & 0.0 & 0.3 & 0.1 & 0.0 & 0.0 & 0.0 \\ 0.0 & 0.0 & 57.5 & 0.0 & 0.0 & 0.0 & 7 . .2 & 76.7 & 0.0 & 0.0 & 0.0 \\ 0.0 & 0.0 & 5.9 & 0.0 & 0.0 & 0.0 & 7.3 & 6.3 & 0.0 & 0.0 & 0.0 \\ 0.0 & 0.0 & 0.0 & 0.0 & 0.0 & 0.0 & 0.0 & 0.0 & 0.0 & 0.0 & 0.0 \\ 0.0 & 0.0 & 25.1 & 0.0 & 0.0 & 0.0 & 11.3 & 15.2 & 0.0 & 0.0 & 0.0\end{array}$


Summer

51 Dearees North Latitude

\begin{tabular}{|c|c|c|c|c|c|c|c|c|c|c|c|c|c|c|c|c|}
\hline WPT & 126 & 122 & 118 & 114 & 110 & $106^{\circ}$ & $\begin{array}{c}\text { earees } \\
102\end{array}$ & $\begin{array}{r}\text { West } \\
98\end{array}$ & $\begin{array}{c}\text { Longit } \\
94\end{array}$ & de & 86 & 82 & 78 & 74 & 70 & 66 \\
\hline $\begin{array}{l}1 \\
2 \\
3 \\
4 \\
5 \\
6 \\
7 \\
8 \\
9\end{array}$ & $\begin{array}{r}0.2 \\
6.9 \\
1.1 \\
30.0 \\
10.0 \\
0.0 \\
3.5 \\
3.8 \\
94.5\end{array}$ & $\begin{array}{r}0.2 \\
4.1 \\
1.0 \\
32.0 \\
15.2 \\
0.0 \\
1.6 \\
4.7 \\
38.3\end{array}$ & $\begin{array}{r}0.2 \\
3.3 \\
1.8 \\
30.2 \\
21.9 \\
0.0 \\
8.2 \\
7.3 \\
27.1\end{array}$ & $\begin{array}{r}1.0 \\
4.0 \\
1.7 \\
31.8 \\
20.7 \\
0.0 \\
9.2 \\
9.1 \\
22.5\end{array}$ & $\begin{array}{r}0.8 \\
6.3 \\
1.6 \\
31.7 \\
21.3 \\
0.0 \\
9.1 \\
11.1 \\
18.2\end{array}$ & $\begin{array}{r}1.0 \\
9.1 \\
2.8 \\
29.1 \\
23.4 \\
0.1 \\
9.1 \\
11.2 \\
15.2\end{array}$ & $\begin{array}{r}2.1 \\
8.1 \\
3.4 \\
29.7 \\
22.7 \\
0.0 \\
7.9 \\
12.7 \\
13.4\end{array}$ & $\begin{array}{r}1.8 \\
11.2 \\
3.8 \\
26.9 \\
22.3 \\
0.0 \\
8.8 \\
13.7 \\
11.5\end{array}$ & $\begin{array}{r}3.1 \\
11.3 \\
2.9 \\
24.3 \\
26.8 \\
0.0 \\
8.5 \\
19.3 \\
8.8\end{array}$ & $\begin{array}{l}0.0 \\
0.0 \\
0.0 \\
0.0 \\
0.0 \\
0.0 \\
0.0 \\
0.0 \\
0.0 \\
0.0\end{array}$ & $\begin{array}{l}0.0 \\
0.0 \\
0.00 \\
0.0 \\
0.0 \\
0.00 \\
0.00 \\
0.00 \\
0.0\end{array}$ & $\begin{array}{l}0.0 \\
0.0 \\
0.0 \\
0.0 \\
0.0 \\
0.0 \\
0.00 \\
0.00 \\
0.0\end{array}$ & $\begin{array}{l}0.0 \\
0.0 \\
0.0 \\
0.0 \\
0.0 \\
0.0 \\
0.0 \\
0.0 \\
0.0\end{array}$ & $\begin{array}{ll}0.0 & 0 \\
0.0 & 0 \\
0.0 & 0 \\
0.0 & 0 \\
0.0 & 0 \\
0.0 & 0 \\
0.0 & 0 \\
0.0 & 0 \\
0.0 & 0\end{array}$ & $\begin{array}{ll}0 & 0 \\
0.0 & 0 \\
0.0 & 0 \\
0.0 & 0 \\
0.0 & 0 \\
0.00 \\
0.0 \\
0.0 \\
0.0\end{array}$ & $\begin{array}{l}0.0 \\
0.0 \\
0.0 \\
0.0 \\
0.0 \\
0.0 \\
0.0 \\
0.0 \\
0.0\end{array}$ \\
\hline
\end{tabular}

48 Deprees North Latitude

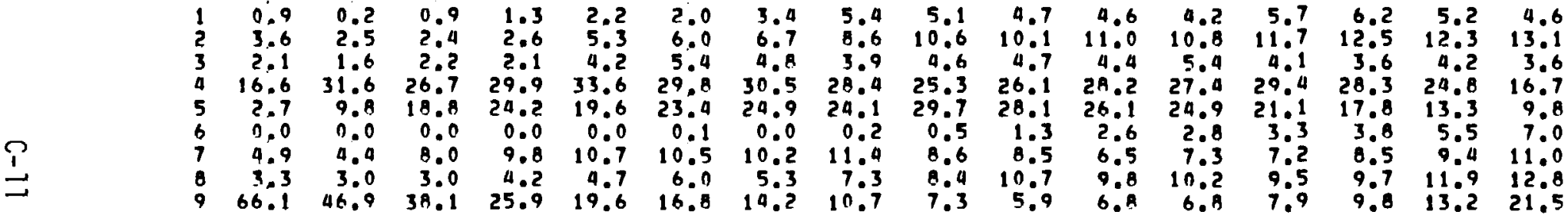

45 Deorees North l.atitude

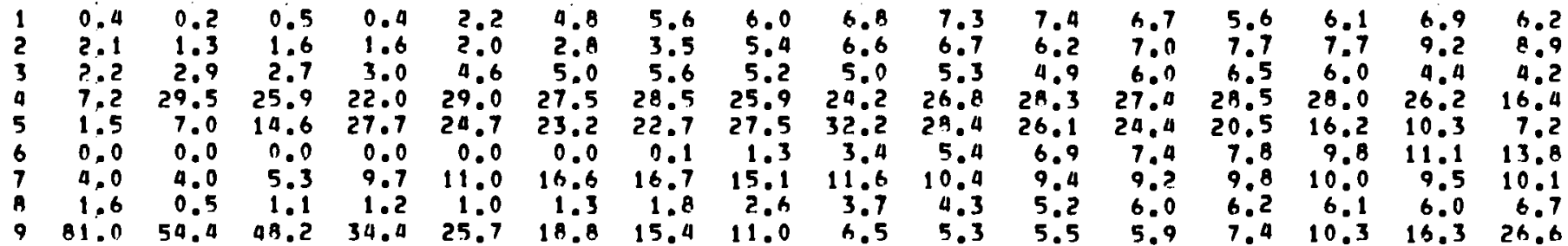

42 Dearees North Latitude

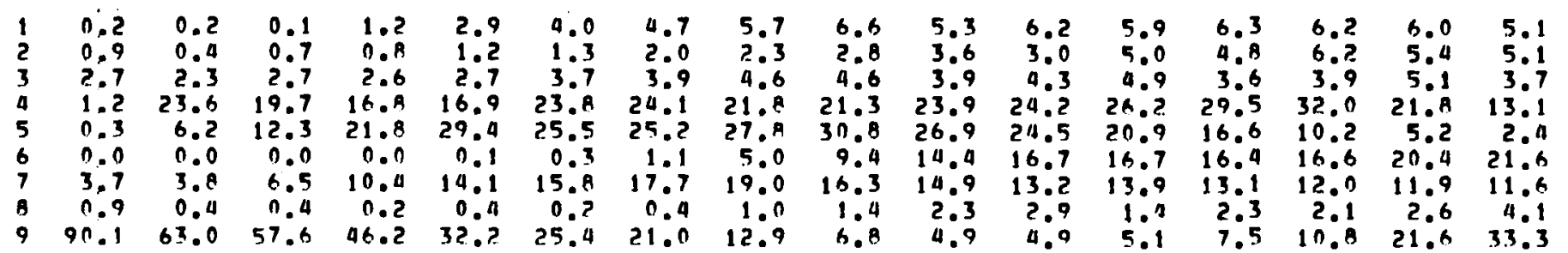


summer

39 Degrees North Latitude

\begin{tabular}{|c|c|c|c|c|c|c|c|c|c|c|c|c|c|c|c|c|}
\hline WPT & 126 & 122 & 118 & 114 & 110 & 106 & $\begin{array}{l}\text { arees } \\
\text { ln? }\end{array}$ & $\begin{array}{r}\text { Wegt } \\
98\end{array}$ & $\begin{array}{c}\text { Longit } \\
94\end{array}$ & dด & 86 & B2 & 78 & 74 & 70 & 66 \\
\hline $\begin{array}{l}1 \\
2 \\
3 \\
4 \\
5 \\
6 \\
7 \\
8 \\
9\end{array}$ & $\begin{array}{r}0.0 \\
0.1 \\
1.2 \\
0.1 \\
0.0 \\
0.0 \\
3.0 \\
0.2 \\
9.8\end{array}$ & $\begin{array}{r}0.1 \\
0.1 \\
1.1 \\
9.4 \\
2.0 \\
0.0 \\
3.7 \\
0.2 \\
83.4\end{array}$ & $\begin{array}{r}0.3 \\
0.2 \\
1.3 \\
12.3 \\
10.3 \\
0.0 \\
4.0 \\
0.0 \\
71.6\end{array}$ & $\begin{array}{r}1.3 \\
0.2 \\
1.3 \\
8.8 \\
21.4 \\
0.0 \\
6.5 \\
0.0 \\
60.5\end{array}$ & $\begin{array}{r}1.5 \\
0.7 \\
1.1 \\
10.8 \\
24.4 \\
0.2 \\
13.3 \\
0.1 \\
47.8\end{array}$ & $\begin{array}{r}1.8 \\
0.7 \\
1.6 \\
19.4 \\
23.1 \\
0.9 \\
16.3 \\
0.1 \\
36.1\end{array}$ & $\begin{array}{r}3.9 \\
0.4 \\
2.8 \\
17.8 \\
23.6 \\
3.7 \\
21.0 \\
0.2 \\
26.5\end{array}$ & $\begin{array}{r}5.3 \\
1.0 \\
2.9 \\
15.5 \\
24.8 \\
15.1 \\
22.1 \\
0.1 \\
13.1\end{array}$ & $\begin{array}{r}4.8 \\
1.0 \\
2.8 \\
15.7 \\
24.1 \\
25.4 \\
19.5 \\
0.1 \\
6.6\end{array}$ & $\begin{array}{r}5.3 \\
1.2 \\
3.3 \\
19.8 \\
18.8 \\
30.0 \\
17.5 \\
0.3 \\
3.8\end{array}$ & $\begin{array}{r}5.5 \\
1.8 \\
2.8 \\
19.3 \\
17.4 \\
31.3 \\
17.1 \\
0.8 \\
3.9\end{array}$ & $\begin{array}{r}4.6 \\
2.0 \\
2.7 \\
20.9 \\
14.6 \\
32.1 \\
17.5 \\
0.3 \\
5.3\end{array}$ & $\begin{array}{r}5.0 \\
2.0 \\
2.5 \\
28.1 \\
7.4 \\
27.7 \\
18.2 \\
0.5 \\
9.7\end{array}$ & $\begin{array}{r}5.2 \\
2.5 \\
3.7 \\
22.8 \\
2.4 \\
30.8 \\
15.2 \\
0.7 \\
16.8\end{array}$ & $\begin{array}{r}5.1 \\
1.6 \\
3.5 \\
13.2 \\
1.2 \\
29.7 \\
15.2 \\
1.4 \\
28.9\end{array}$ & 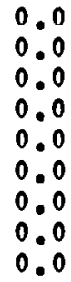 \\
\hline
\end{tabular}

36 Deorees North Latitude

$\begin{array}{rrrrrrrrrrr}0.9 & 2.2 & 2.1 & 2.1 & 2.9 & 2.9 & 2.8 & 3.0 & 3.0 & 0.0 & 0.0 \\ 0.2 & 0.1 & 0.2 & 0.1 & 0.4 & 0.5 & 0.5 & 0.7 & 0.5 & 0.0 & 0.0 \\ 0.4 & 0.8 & 0.5 & 0.5 & 1.8 & 1.2 & 1.3 & 1.6 & 1.4 & 0.0 & 0.0 \\ 14.4 & 11.2 & 9.5 & 11.8 & 13.2 & 12.9 & 15.4 & 17.4 & 10.8 & 0.0 & 0.0 \\ 20.6 & 20.1 & 18.8 & 11.4 & 9.9 & 9.3 & 7.4 & 2.4 & 0.4 & 0.0 & 0.0 \\ 1.5 & 14.0 & 39.6 & 51.4 & 53.5 & 52.5 & 48.7 & 45.9 & 44.0 & 0.0 & 0.0 \\ 9.1 & 17.1 & 17.9 & 15.9 & 15.2 & 16.1 & 16.4 & 17.1 & 16.4 & 0.0 & 0.0 \\ 0.0 & 0.0 & 0.1 & 0.1 & 0.0 & 0.1 & 0.1 & 0.1 & 0.3 & 0.0 & 0.0 \\ 52.8 & 34.6 & 11.3 & 3.6 & 3.0 & 4.4 & 6.9 & 11.9 & 20.9 & 0.0 & 0.0\end{array}$

33 Dearaes North Latitude

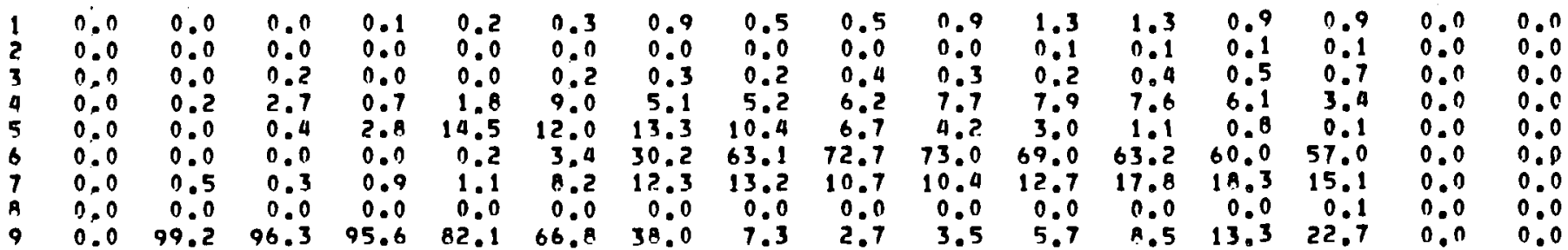

30 Dearees North Lotitude

\begin{tabular}{|c|c|c|c|c|c|c|c|c|c|c|c|c|c|c|}
\hline $\begin{array}{ll}0 & 0 \\
0.0 & 0 \\
0.0 & 0 \\
0.0 & 0 \\
0.0 & 0 \\
0.0 & 0 \\
0.0 & 0 \\
0.0 & 0 \\
0.0 & 0\end{array}$ & $\begin{array}{l}0.0 \\
0.0 \\
0.0 \\
0.0 \\
0.0 \\
0.0 \\
0.0 \\
0.0 \\
0.0\end{array}$ & $\begin{array}{r}0.0 \\
0.0 \\
0.1 \\
0.5 \\
0.0 \\
0.0 \\
0.1 \\
0.0 \\
99.2\end{array}$ & $\begin{array}{l}0.0 \\
0.0 \\
0.0 \\
1.1 \\
0.7 \\
0.0 \\
0.0 \\
0.0 \\
9.0\end{array}$ & $\begin{array}{r}0.0 \\
0.0 \\
0.0 \\
0.5 \\
2.4 \\
0.1 \\
0.5 \\
0.0 \\
96.4\end{array}$ & $\begin{array}{r}0.0 \\
0.0 \\
0.1 \\
2.0 \\
0.2 \\
1.0 \\
3.7 \\
0.0 \\
8.9\end{array}$ & $\begin{array}{r}0.2 \\
0.0 \\
0.0 \\
2.1 \\
0.6 \\
34.9 \\
6.1 \\
0.0 \\
49.2\end{array}$ & $\begin{array}{rl}0 . & 0 \\
0.0 & 0 \\
0.0 \\
3.0 \\
5.1 \\
81.1 \\
4 . \\
0.0 \\
5.5\end{array}$ & $\begin{array}{r}0.1 \\
0.0 \\
0.3 \\
4.4 \\
1.3 \\
85.7 \\
4.7 \\
0.0 \\
3.5\end{array}$ & $\begin{array}{r}0.1 \\
0.0 \\
0.1 \\
3.1 \\
0.7 \\
84.7 \\
6.5 \\
0.0 \\
4.8\end{array}$ & $\begin{array}{r}0.3 \\
0.0 \\
0.0 \\
3.1 \\
0.11 \\
81.3 \\
8.8 \\
0.0 \\
6.0\end{array}$ & $\begin{array}{r}0.1 \\
0.0 \\
0.1 \\
2.1 \\
0.1 \\
80.6 \\
9.2 \\
0.0 \\
7.7\end{array}$ & $\begin{array}{r}0.1 \\
0.0 \\
0.0 \\
1.5 \\
0.0 \\
76.0 \\
8.9 \\
0.0 \\
12.7\end{array}$ & $\begin{array}{l}0.0 \\
0.0 \\
0.0 \\
0.0 \\
0.0 \\
0.0 \\
0.0 \\
0.0 \\
0.0 \\
0.0\end{array}$ & $\begin{array}{l}0.0 \\
0.0 \\
0.0 \\
0.0 \\
0.0 \\
0.0 \\
0.0 \\
0.0 \\
0.0\end{array}$ \\
\hline
\end{tabular}


Summer

27 Dearees North Latitude

\begin{tabular}{|c|c|c|c|c|c|c|c|c|c|c|c|c|c|c|c|c|}
\hline WPT & 126 & 122 & 118 & 114 & 110 & 106 & $\begin{array}{l}\text { arees } \\
102\end{array}$ & $\begin{array}{r}\text { Weat } \\
\text { QA }\end{array}$ & $\begin{array}{c}\text { Longlt } \\
94\end{array}$ & ut de & 86 & 82 & 78 & 74 & 70 & 66 \\
\hline $\begin{array}{l}1 \\
2 \\
3 \\
4 \\
5 \\
6 \\
7 \\
8 \\
9\end{array}$ & $\begin{array}{l}0.0 \\
0.0 \\
0.0 \\
0.0 \\
0.0 \\
0.0 \\
0.0 \\
0.0 \\
0.0 \\
0.0\end{array}$ & $\begin{array}{l}0.0 \\
0.0 \\
0.0 \\
0.0 \\
0.0 \\
0.0 \\
0.0 \\
0.0 \\
0.0\end{array}$ & $\begin{array}{l}0.0 \\
0.0 \\
0.0 \\
0.0 \\
0.0 \\
0.0 \\
0.0 \\
0.0 \\
0.0\end{array}$ & $\begin{array}{l}0.0 \\
0.0 \\
0.0 \\
0.0 \\
0.0 \\
0.0 \\
0.0 \\
0.0 \\
0.0\end{array}$ & $\begin{array}{l}0.0 \\
0.0 \\
0.0 \\
0.0 \\
0.0 \\
0.0 \\
0.0 \\
0.0 \\
0.0 \\
0.0\end{array}$ & $\begin{array}{l}0.0 \\
0.0 \\
0.0 \\
0.0 \\
0.0 \\
0.0 \\
0.0 \\
0.0 \\
0.0 \\
0.0\end{array}$ & $\begin{array}{r}0.0 \\
0.0 \\
0.0 \\
0.2 \\
3.3 \\
20.4 \\
2.3 \\
0.0 \\
73.9\end{array}$ & $\begin{array}{r}0.1 \\
0.0 \\
0.0 \\
1.5 \\
2.4 \\
83.4 \\
2.7 \\
0.0 \\
9.9\end{array}$ & $\begin{array}{r}0.1 \\
0.0 \\
0.0 \\
1.5 \\
0.3 \\
89.7 \\
2.5 \\
0.0 \\
5.9\end{array}$ & $\begin{array}{r}0.0 \\
0.0 \\
0.1 \\
0.7 \\
0.2 \\
90.8 \\
1.4 \\
0.0 \\
6.8\end{array}$ & $\begin{array}{r}0.0 \\
0.0 \\
0.0 \\
0.7 \\
0.0 \\
90.1 \\
1.7 \\
0.0 \\
7.5\end{array}$ & $\begin{array}{r}0.0 \\
0.0 \\
0.0 \\
0.4 \\
0.0 \\
89.9 \\
7.3 \\
0.0 \\
7.4\end{array}$ & $\begin{array}{r}0=0 \\
0.0 \\
0.0 \\
0.2 \\
0.0 \\
87.0 \\
1.8 \\
0.0 \\
10.3\end{array}$ & $\begin{array}{l}0.0 \\
0.0 \\
0.0 \\
0.0 \\
0.0 \\
0.0 \\
0.0 \\
0.0 \\
0.0 \\
0.0\end{array}$ & $\begin{array}{l}0.0 \\
0.0 \\
0.0 \\
0.0 \\
0.0 \\
0.0 \\
0.0 \\
0.0 \\
0.0\end{array}$ & $\begin{array}{l}0.0 \\
0.0 \\
0.0 \\
0.0 \\
0.0 \\
0.0 \\
0.0 \\
0.0 \\
0.00 \\
0.0\end{array}$ \\
\hline
\end{tabular}

24 Dearees North Latitude

$\begin{array}{llllll}1 & 0.0 & 0.0 & 0.0 & 0.0 & 0.0 \\ ? & 0.0 & 0.0 & 0.0 & 0.0 & 0.0 \\ 3 & 0.0 & 0.0 & 0.0 & 0.0 & 0.0 \\ 4 & 0.0 & 0.0 & 0.0 & 0.0 & 0.0 \\ 5 & 0.0 & 0.0 & 0.0 & 0.0 & 0.0 \\ 6 & 0.0 & 0.0 & 0.0 & 0.0 & 0.0 \\ 7 & 0.0 & 0.0 & 0.0 & 0.0 & 0.0 \\ 9 & 0.0 & 0.0 & 0.0 & 0.0 & 0.0 \\ 9 & 0.0 & 0.0 & 0.0 & 0.0 & 0.0\end{array}$

0.0
0.0
0.0
0.0
0.0
0.0
0.0
0.0
0.0

$\begin{array}{rr}0.0 & 0.0 \\ 0.0 & 0.0 \\ 0.0 & 0.0 \\ 0.0 & 0.0 \\ 0.0 & 2.0 \\ 0.0 & 71.6 \\ 0.0 & 0.0 \\ 0.0 & 0.0 \\ 0.0 & 24\end{array}$

0.0
0.0
0.0
0.9
2.3
71.6
0.9
0.0
24.4

$\begin{array}{ll}0.0 & 0.0 \\ 0.0 & 0.0 \\ 0.0 & 0.0 \\ 0.0 & 0.0 \\ 0.0 & 0.0 \\ 0.0 & 0.0 \\ 0.0 & 0.0 \\ 0.0 & 0.0 \\ 0.0 & 0.0\end{array}$

$\begin{array}{rrrr}0.0 & 0.0 & 0.0 & 0.0 \\ 0.0 & 0.0 & 0.0 & 0.0 \\ 0.0 & 0.0 & 0.0 & 0.0 \\ 0.0 & 0.0 & 0.1 & 0.0 \\ 0.0 & 0.0 & 0.0 & 0.0 \\ 0.0 & 90.0 & 91.2 & 0.0 \\ 0.0 & 0.2 & 0.4 & 0.0 \\ 0.0 & 0.0 & 0.0 & 0.0 \\ 0.0 & 9.8 & 0.2 & 0.0\end{array}$

$\begin{array}{ll}0.0 & 0.0 \\ 0.0 & 0.0 \\ 0.0 & 0.0 \\ 0.0 & 0.0 \\ 0.0 & 0.0 \\ 0.0 & 0.0 \\ 0.0 & 0.0 \\ 0.0 & 0.0 \\ 0.0 & 0.0\end{array}$


Autumn

51 Deqrees North Latitude

\begin{tabular}{|c|c|c|c|c|c|c|c|c|c|c|c|c|c|c|c|c|}
\hline WPT & 126 & 122 & 118 & 114 & 110 & 106 & $\begin{array}{l}\text { areas } \\
102\end{array}$ & $\begin{array}{r}\text { Weat } \\
\text { QB }\end{array}$ & $\begin{array}{c}\text { Longlt } \\
94\end{array}$ & $\begin{array}{l}\text { tde } \\
90\end{array}$ & 86 & 82 & 78 & 74 & 70 & 66 \\
\hline $\begin{array}{l}1 \\
2 \\
3 \\
4 \\
5 \\
6 \\
7 \\
9 \\
9\end{array}$ & $\begin{array}{r}2.6 \\
17.5 \\
2.4 \\
12.6 \\
20.6 \\
0.0 \\
6.6 \\
8.2 \\
29.0\end{array}$ & $\begin{array}{r}1.9 \\
13.5 \\
2.5 \\
19.5 \\
30.2 \\
0.0 \\
7.5 \\
5.1 \\
19.1\end{array}$ & $\begin{array}{r}2.0 \\
9.5 \\
2.6 \\
32.2 \\
21.6 \\
0.0 \\
12.3 \\
5.6 \\
14.1\end{array}$ & $\begin{array}{r}3.1 \\
7.2 \\
3.5 \\
30.2 \\
18.9 \\
0.0 \\
14.1 \\
6.9 \\
16.1\end{array}$ & $\begin{array}{r}3.9 \\
8.7 \\
3.2 \\
28.7 \\
20.1 \\
0.0 \\
12.2 \\
8.3 \\
15.0\end{array}$ & $\begin{array}{r}3.0 \\
11.6 \\
2.6 \\
29.1 \\
23.2 \\
9.0 \\
9.2 \\
9.7 \\
11.7\end{array}$ & $\begin{array}{r}2.8 \\
12.3 \\
3.0 \\
29.8 \\
22.3 \\
0.0 \\
8.7 \\
11.9 \\
9.3\end{array}$ & $\begin{array}{r}2.4 \\
14.2 \\
3.0 \\
29.7 \\
20.5 \\
0.0 \\
8.2 \\
14.6 \\
7.5\end{array}$ & $\begin{array}{r}2.8 \\
15.1 \\
3.5 \\
26.7 \\
23.4 \\
0.0 \\
6.1 \\
14.8 \\
7.7\end{array}$ & 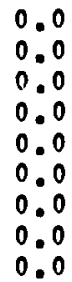 & $\begin{array}{l}0.0 \\
0.0 \\
0.0 \\
0.0 \\
0.0 \\
0.0 \\
0.0 \\
0.0 \\
0.0\end{array}$ & $\begin{array}{l}0.0 \\
0.0 \\
0.0 \\
0.0 \\
0.0 \\
0.0 \\
0.0 \\
0.0 \\
0.0\end{array}$ & $\begin{array}{l}0.0 \\
0.0 \\
0.0 \\
0.0 \\
0.0 \\
0.0 \\
0.0 \\
0.0 \\
0.0 \\
0.0\end{array}$ & $\begin{array}{l}0.0 \\
0.0 \\
0.0 \\
0.0 \\
0.0 \\
0.0 \\
0.0 \\
0.0 \\
0.0\end{array}$ & $\begin{array}{l}0.0 \\
0.00 \\
0.0 \\
0.0 \\
0.0 \\
0.0 \\
0.0 \\
0.0 \\
0.0 \\
0.0\end{array}$ & 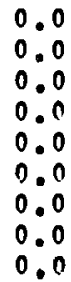 \\
\hline
\end{tabular}

4A Dearees North Lotitude

\begin{tabular}{|c|c|c|c|c|c|c|c|c|c|c|c|c|c|c|c|}
\hline $\begin{array}{r}10.0 \\
0.0 \\
6.0 \\
9.9 \\
50.5\end{array}$ & $\begin{array}{r}2.0 \\
12.8 \\
2.6 \\
17.6 \\
29.2 \\
0.0 \\
6.0 \\
4.5 \\
25.2\end{array}$ & $\begin{array}{r}1.7 \\
8.4 \\
2.9 \\
24.1 \\
32.9 \\
0.0 \\
9.5 \\
4.0 \\
16.6\end{array}$ & $\begin{array}{r}3.4 \\
5.2 \\
3.1 \\
35.1 \\
20.5 \\
0.0 \\
14.3 \\
4.3 \\
14.2\end{array}$ & $\begin{array}{r}4.5 \\
6.2 \\
3.5 \\
30.9 \\
18.0 \\
0.0 \\
16.2 \\
5.0 \\
15.0\end{array}$ & $\begin{array}{r}4.1 \\
8.4 \\
3.5 \\
28.8 \\
21.7 \\
0.0 \\
12.8 \\
7.7 \\
13.0\end{array}$ & $\begin{array}{r}3.9 \\
9.5 \\
4.7 \\
28.7 \\
21.9 \\
0.0 \\
9.8 \\
9.4 \\
12.1\end{array}$ & $\begin{array}{r}4.3 \\
11.4 \\
4.3 \\
27.7 \\
22.9 \\
0.0 \\
8.6 \\
12.1 \\
8.7\end{array}$ & $\begin{array}{r}5.6 \\
12.6 \\
3.6 \\
26.2 \\
24.6 \\
0.6 \\
7.6 \\
12.0 \\
7.3\end{array}$ & $\begin{array}{r}6.0 \\
14.4 \\
3.2 \\
28.0 \\
23.0 \\
0.9 \\
6.2 \\
12.7 \\
5.0\end{array}$ & $\begin{array}{r}5.6 \\
15.2 \\
2.0 \\
27.0 \\
24.3 \\
1.3 \\
5.6 \\
14.0 \\
4.5\end{array}$ & $\begin{array}{r}4.4 \\
16.8 \\
2.9 \\
25.6 \\
24.6 \\
2.0 \\
5.4 \\
10.1 \\
0.3\end{array}$ & $\begin{array}{r}9.5 \\
15.5 \\
2.9 \\
25.9 \\
24.8 \\
2.8 \\
5.2 \\
14.8 \\
3.6\end{array}$ & $\begin{array}{r}3 . \\
14 . \\
3 . \\
27 \\
22 . \\
3 . \\
3 . \\
16 . \\
5 .\end{array}$ & $\begin{array}{r}3.7 \\
14.3 \\
3.1 \\
27.5 \\
17.2 \\
4.4 \\
4.7 \\
17.5 \\
7.5\end{array}$ & $\begin{array}{r}3.4 \\
15.2 \\
1.9 \\
25.0 \\
11.7 \\
6.2 \\
5.0\end{array}$ \\
\hline
\end{tabular}

45 Dearees North Letitude

\begin{tabular}{|c|c|c|c|c|c|c|c|c|c|c|c|c|c|c|c|}
\hline $\begin{array}{r}2.1 \\
6.2 \\
3.6 \\
3.4 \\
9.6 \\
0.0 \\
5.5 \\
7.1 \\
67.5\end{array}$ & $\begin{array}{r}1.8 \\
6.6 \\
3.5 \\
16.1 \\
29.8 \\
0.0 \\
7.1 \\
4.4 \\
31.8\end{array}$ & $\begin{array}{r}1.4 \\
5.4 \\
2.6 \\
21.6 \\
36.3 \\
0.0 \\
7.7 \\
3.0 \\
21.9\end{array}$ & $\begin{array}{r}2.0 \\
3.6 \\
3.7 \\
29.8 \\
31.6 \\
0.0 \\
10.4 \\
2.3 \\
16.5\end{array}$ & $\begin{array}{r}4.3 \\
5.0 \\
2.9 \\
37.7 \\
19.7 \\
0.0 \\
12.5 \\
1.9 \\
16.1\end{array}$ & $\begin{array}{r}5.4 \\
6.0 \\
3.4 \\
30.7 \\
19.2 \\
0.0 \\
15.4 \\
3.3 \\
16.6\end{array}$ & $\begin{array}{r}5.2 \\
6.5 \\
3.6 \\
29.3 \\
23.3 \\
0.1 \\
12.1 \\
5.4 \\
14.4\end{array}$ & $\begin{array}{r}4.9 \\
8.2 \\
5.1 \\
28.4 \\
24.4 \\
0.3 \\
9.6 \\
7.8 \\
11.4\end{array}$ & $\begin{array}{r}5.5 \\
11.1 \\
4.9 \\
27.2 \\
27.6 \\
1.3 \\
8.3 \\
7.2 \\
6.9\end{array}$ & $\begin{array}{r}5.3 \\
10.7 \\
3.3 \\
30.0 \\
26.4 \\
3.2 \\
7.2 \\
9.8 \\
4.2\end{array}$ & $\begin{array}{r}6.2 \\
10.9 \\
4.1 \\
26.0 \\
26.2 \\
3.7 \\
7.9 \\
11.7 \\
3.7\end{array}$ & $\begin{array}{r}5.6 \\
12.7 \\
4.2 \\
24.7 \\
25.8 \\
4.5 \\
9.3 \\
11.2\end{array}$ & & $\begin{array}{l}4.4 \\
3.1 \\
3.6 \\
5.0\end{array}$ & $\begin{array}{r}4.4 \\
12.1 \\
3.5 \\
26.6 \\
22.5 \\
6.0 \\
8.3 \\
12.8 \\
3.9\end{array}$ & $\begin{array}{r}4.0 \\
12.6 \\
3.3 \\
30.9 \\
13.5 \\
7.9 \\
6.4 \\
14.2 \\
7.3\end{array}$ \\
\hline
\end{tabular}

42 Dearees North Letitude

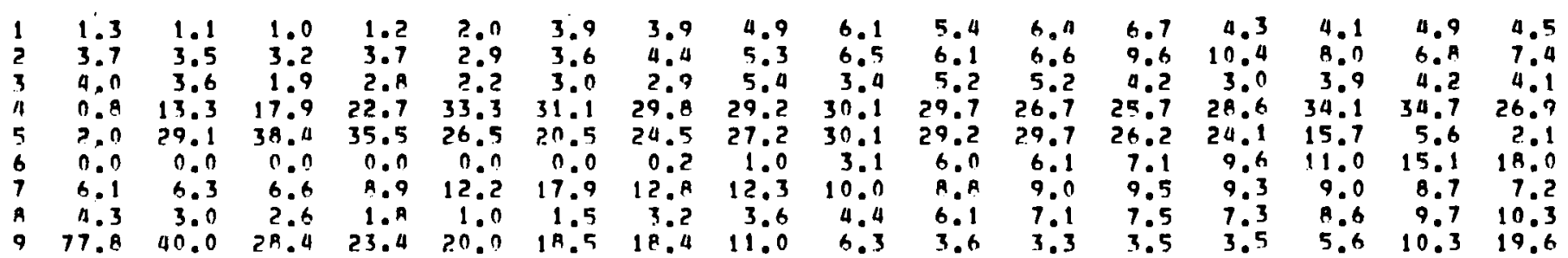


Autumn

39 Dsarees North Lotitude

\begin{tabular}{|c|c|c|c|c|c|c|c|c|c|c|c|c|c|c|c|c|}
\hline WPT & 126 & 122 & 118 & 114 & 110 & $106^{\circ}$ & $\begin{array}{c}\text { dearees } \\
\text { in? }\end{array}$ & $\begin{array}{r}\text { We et } \\
98\end{array}$ & $\underset{94}{\text { Longit }}$ & tde & 86 & 82 & 78 & 74 & 70 & \\
\hline $\begin{array}{l}1 \\
? \\
3 \\
4 \\
5 \\
6 \\
7 \\
8 \\
9\end{array}$ & $\begin{array}{r}1.3 \\
1.2 \\
3.0 \\
0.0 \\
0.7 \\
0.0 \\
5.1 \\
2.6 \\
86.1\end{array}$ & $\begin{array}{r}0.2 \\
1.5 \\
2.5 \\
5.6 \\
16.6 \\
0.0 \\
4.7 \\
1.8 \\
66.9\end{array}$ & $\begin{array}{r}0.0 \\
2.2 \\
1.9 \\
12.6 \\
37.3 \\
0.0 \\
5.6 \\
1.9 \\
38.1\end{array}$ & $\begin{array}{r}1.1 \\
2.5 \\
1.7 \\
16.4 \\
39.6 \\
0.0 \\
7.4 \\
1.4 \\
30.9\end{array}$ & $\begin{array}{r}1.2 \\
1.8 \\
1.7 \\
22.3 \\
35.3 \\
0.0 \\
10.4 \\
1.1 \\
26.4\end{array}$ & $\begin{array}{r}2.1 \\
2.4 \\
2.4 \\
34.4 \\
21.4 \\
0.0 \\
15.4 \\
1.2 \\
20.6\end{array}$ & $\begin{array}{r}3.4 \\
3.6 \\
3.6 \\
23.8 \\
26.5 \\
0.3 \\
16.6 \\
1.8 \\
20.3\end{array}$ & $\begin{array}{r}4.9 \\
5.1 \\
2.9 \\
25.7 \\
27.9 \\
3.3 \\
15.3 \\
2.4 \\
12.6\end{array}$ & $\begin{array}{r}5.0 \\
4.4 \\
3.2 \\
27.7 \\
29.9 \\
9.3 \\
12.9 \\
3.0 \\
4.7\end{array}$ & $\begin{array}{r}4.1 \\
4.3 \\
4.9 \\
28.0 \\
27.9 \\
12.5 \\
11.7 \\
2.6 \\
1.1\end{array}$ & $\begin{array}{r}4.6 \\
5.1 \\
4.4 \\
28.0 \\
27.5 \\
14.1 \\
10.0 \\
3.3 \\
3.0\end{array}$ & $\begin{array}{r}5.0 \\
5.8 \\
4.2 \\
24.6 \\
26.4 \\
15.1 \\
8.3 \\
2.9 \\
3.9\end{array}$ & $\begin{array}{r}4.4 \\
6.1 \\
3.5 \\
34.4 \\
16.0 \\
16.6 \\
9.3 \\
9.0 \\
5.7\end{array}$ & $\begin{array}{r}4.7 \\
4.3 \\
4.5 \\
36.1 \\
5.3 \\
20.4 \\
9.9 \\
4.5 \\
10.3\end{array}$ & $\begin{array}{r}4.3 \\
3.7 \\
4.9 \\
27.8 \\
1.4 \\
25.5 \\
7.0 \\
6.0 \\
19.1\end{array}$ & \\
\hline
\end{tabular}

36 Deareas North Latitude

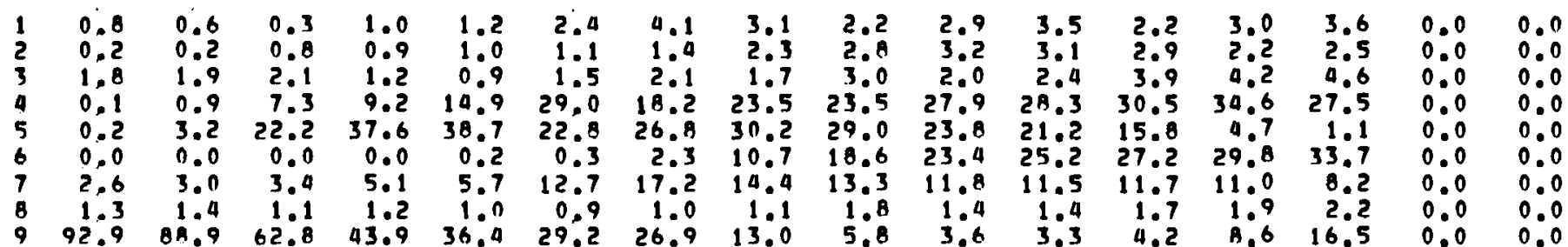

33 Deorees North Letitude

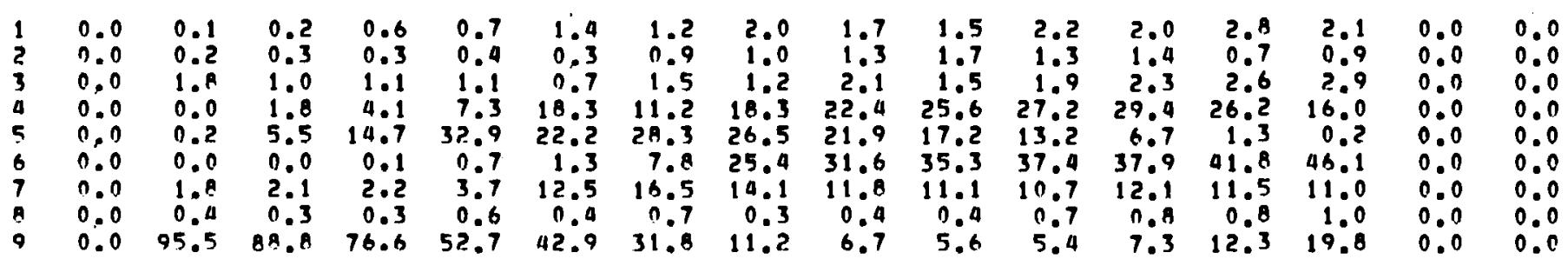

30 Donress North Latitude

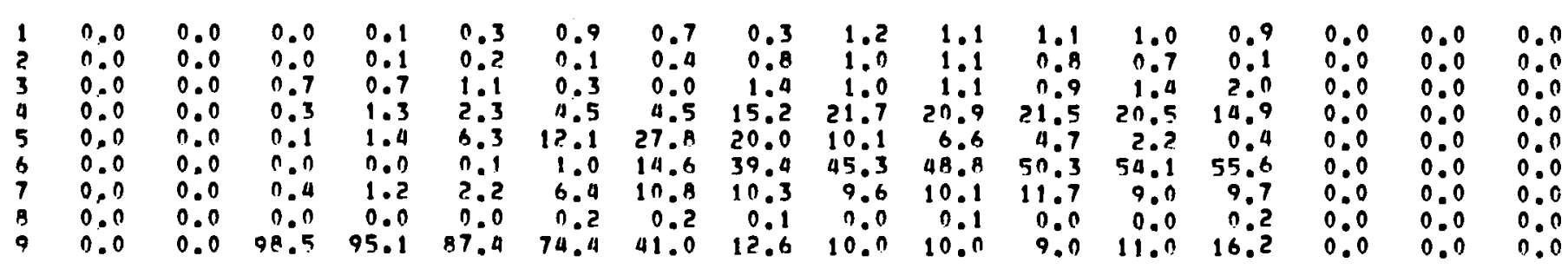


Autumn

27 Deareas North Letitude

\begin{tabular}{|c|c|c|c|c|c|}
\hline & 126 & 122 & 118 & 114 & 110 \\
\hline $\begin{array}{l}1 \\
2 \\
3 \\
4 \\
5 \\
6 \\
7 \\
9 \\
9\end{array}$ & $\begin{array}{l}0.0 \\
0.0 \\
0.0 \\
0.0 \\
0.0 \\
0.0 \\
0.0 \\
0.0 \\
0.0\end{array}$ & $\begin{array}{l}0.0 \\
0.0 \\
0.0 \\
0.0 \\
0.0 \\
0.0 \\
0.0 \\
0.0 \\
0.0\end{array}$ & $\begin{array}{l}0.0 \\
0.0 \\
0.0 \\
0.0 \\
0.0 \\
0.0 \\
0.0 \\
0.0 \\
0.0 \\
0.0\end{array}$ & $\begin{array}{l}0.0 \\
0.0 \\
0.0 \\
0.0 \\
0.0 \\
0.0 \\
0.0 \\
0.0 \\
0.0 \\
0.0\end{array}$ & $\begin{array}{l}0.0 \\
0.0 \\
0.0 \\
0.0 \\
0.0 \\
0.0 \\
0.0 \\
0.0 \\
0.0\end{array}$ \\
\hline
\end{tabular}

Deorees West Lonoltutde

\begin{tabular}{|c|c|c|c|c|c|c|c|c|}
\hline 102 & 98 & 94 & 90 & 86 & $B 2$ & 78 & 74 & 70 \\
\hline $\begin{array}{r}0.2 \\
0.2 \\
0.0 \\
1.9 \\
15.2 \\
9.6 \\
5.6 \\
0.0 \\
67.3\end{array}$ & $\begin{array}{r}0.6 \\
0.1 \\
0.6 \\
12.3 \\
12.1 \\
47.7 \\
8.3 \\
0.0 \\
18.3\end{array}$ & $\begin{array}{r}0.4 \\
0.2 \\
0.7 \\
14.6 \\
3.5 \\
54.5 \\
10.7 \\
0.0 \\
15.4\end{array}$ & $\begin{array}{r}1.0 \\
0.3 \\
0.4 \\
12.5 \\
1.1 \\
58.4 \\
10.4 \\
0.0 \\
15.9\end{array}$ & $\begin{array}{r}0.4 \\
0.2 \\
0.6 \\
10.9 \\
0.8 \\
62.1 \\
9.5 \\
0.0 \\
15.5\end{array}$ & $\begin{array}{r}0.6 \\
0.2 \\
0.8 \\
9.9 \\
0.1 \\
63.4 \\
0.9 \\
0.0 \\
16.1\end{array}$ & $\begin{array}{r}0.3 \\
0.0 \\
0.6 \\
6.5 \\
0.0 \\
64.5 \\
9.2 \\
0.1 \\
18.9\end{array}$ & $\begin{array}{l}0.0 \\
0.0 \\
0.0 \\
0.0 \\
0.0 \\
0.0 \\
0.0 \\
0.0 \\
0.0 \\
0.0\end{array}$ & $\begin{array}{ll}0.0 & 0 \\
0 & 0 \\
0.0 & 0 \\
0.0 & 0 \\
0.0 & 0 \\
0.0 & 0 \\
0.0 & 0 \\
0.0 & 0 \\
0.0 & 0\end{array}$ \\
\hline
\end{tabular}

24 Deareas North Lotitude

$\begin{array}{llllll}1 & 0.0 & 0.0 & 0.0 & 0.0 & 0.0 \\ ? & 0.0 & 0.0 & 0.0 & 0.0 & 0.0 \\ 3 & 0.0 & 0.0 & 0.0 & 0.0 & 0.0 \\ 3 & 0.0 & 0.0 & 0.0 & 0.0 & 0.0 \\ 5 & 0.0 & 0.0 & 0.0 & 0.0 & 0.0 \\ 6 & 0.0 & 0.0 & 0.0 & 0.0 & 0.0 \\ 7 & 0.0 & 0.0 & 0.0 & 0.0 & 0.0 \\ 3 & 0.0 & 0.0 & 0.0 & 0.0 & 0.0 \\ 9 & 0.0 & 0.0 & 0.0 & 0.0 & 0.0\end{array}$

0.0
0.0
0.0
0.0
0.0
0.0
0.0
0.0
0.0

$\begin{array}{rrrr}0.0 & 0.0 & 0.0 & 0.0 \\ 0.0 & 0.0 & 0.0 & 0.0 \\ 0.0 & 0.3 & 0.0 & 0.0 \\ 0.0 & 9.3 & 0.0 & 0.0 \\ 0.0 & 7.9 & 0.0 & 0.0 \\ 0.0 & 44.4 & 0.0 & 0.0 \\ 0.0 & 4.6 & 0.0 & 0.0 \\ 0.0 & 0.0 & 0.0 & 0.0 \\ 0.0 & 32.7 & 0.0 & 0.0\end{array}$

$\begin{array}{rrrrrr}0.0 & 0.0 & 0.0 & 0.0 & 0.0 & 0.0 \\ 0.0 & 0.1 & 0.0 & 0.0 & 0.0 & 0.0 \\ 0.0 & 0.4 & 0.1 & 0.0 & 0.0 & 0.0 \\ 0.0 & 3.5 & 2.6 & 0.0 & 0.0 & 0.0 \\ 0.0 & 0.1 & 0.0 & 0.0 & 0.0 & 0.0 \\ 0.0 & 63.9 & 65.2 & 0.0 & 0.0 & 0.0 \\ 0.0 & 7.2 & 6.8 & 0.0 & 0.0 & 0.0 \\ 0.0 & 0.0 & 0.0 & 0.0 & 0.0 & 0.0 \\ 0.0 & 24.7 & 25.2 & 0.0 & 0.0 & 0.0\end{array}$


1969

51 Dearees North Lattude

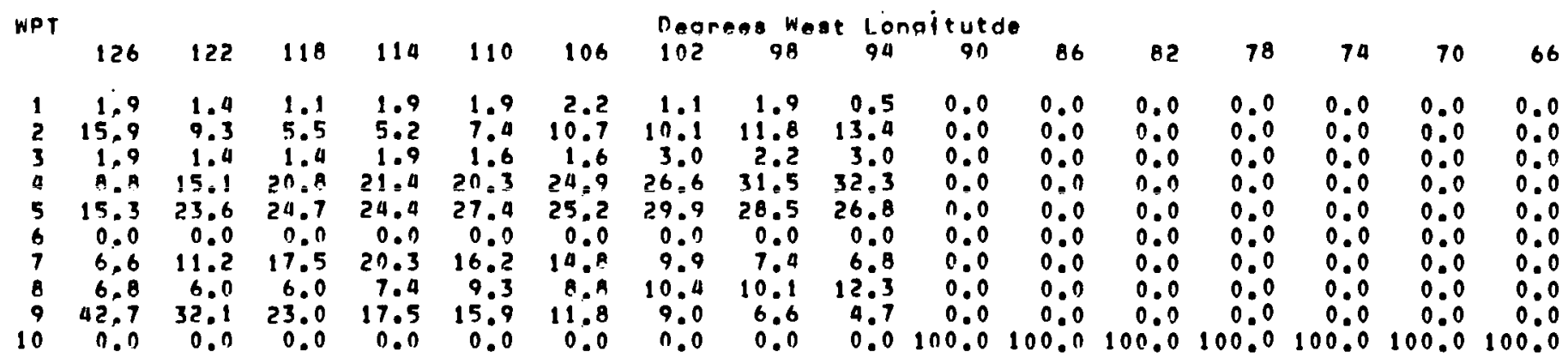

4A Degrees North Latitude

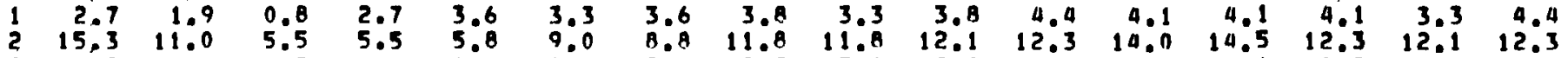

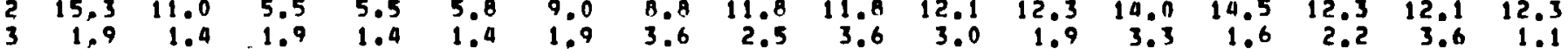
$\begin{array}{rrrrrrrrrrrrrrrrr}3 & 1.9 & 1.4 & 1.9 & 1.4 & 1.4 & 1.9 & 3.6 & 2.5 & 3.6 & 3.0 & 1.9 & 3.3 & 1.6 & 2.2 & 3.6 & 1.1\end{array}$

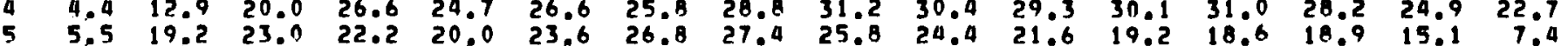

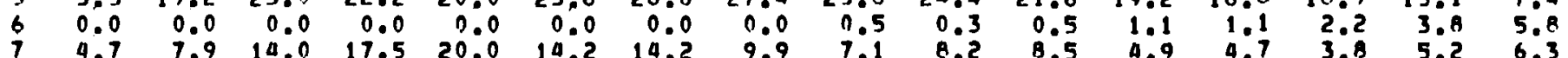

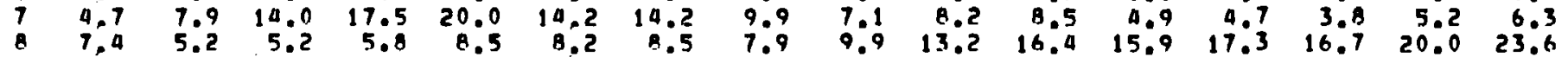

45 Dearees North Lotitude

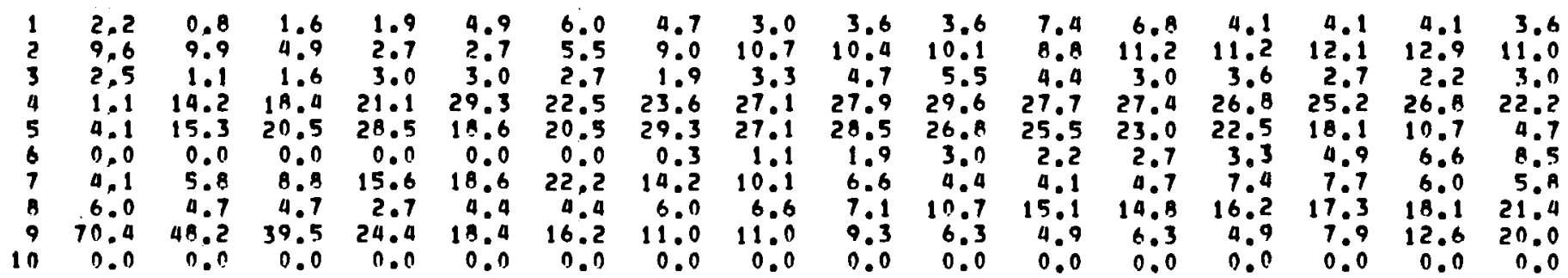

42 Dearees North Let t tude

\begin{tabular}{|c|c|c|c|c|c|c|c|c|c|c|c|c|c|c|c|c|}
\hline $\begin{array}{l}1 \\
2 \\
3 \\
4 \\
5 \\
6 \\
7 \\
6\end{array}$ & $\begin{array}{r}1.6 \\
5.5 \\
3.3 \\
0.3 \\
1.4 \\
0.0 \\
4.0 \\
4.4 \\
79.2 \\
0.0\end{array}$ & $\begin{array}{r}1.9 \\
5.8 \\
1.9 \\
11.2 \\
16.4 \\
0.0 \\
5.8 \\
4.1 \\
52.9 \\
0.0\end{array}$ & $\begin{array}{r}3.3 \\
3.3 \\
1.6 \\
13.7 \\
22.5 \\
0.0 \\
6.6 \\
7.7 \\
46.3 \\
0.0\end{array}$ & $\begin{array}{r}2.7 \\
4.1 \\
2.7 \\
16.7 \\
28.5 \\
0.0 \\
11.8 \\
2.7 \\
30.7 \\
0.0\end{array}$ & $\begin{array}{r}2.7 \\
2.7 \\
1.9 \\
27.7 \\
21.6 \\
0.0 \\
17.8 \\
2.5 \\
23.0 \\
0.0\end{array}$ & $\begin{array}{r}3.8 \\
3.6 \\
1.0 \\
25.5 \\
20.9 \\
0.3 \\
23.6 \\
3.0 \\
18.1 \\
0.0\end{array}$ & $\begin{array}{r}3.0 \\
6.3 \\
2.2 \\
23.6 \\
28.5 \\
1.1 \\
17.2 \\
1.6 \\
15.9 \\
0.0\end{array}$ & $\begin{array}{r}3.3 \\
6.3 \\
4.1 \\
26.3 \\
28.3 \\
3.8 \\
11.5 \\
3.4 \\
12.9 \\
0.0\end{array}$ & $\begin{array}{r}4.7 \\
6.6 \\
4.4 \\
27.4 \\
27.9 \\
5.2 \\
9.6 \\
4.1 \\
10.1 \\
0.0\end{array}$ & $\begin{array}{r}4.9 \\
7.9 \\
6.6 \\
27.7 \\
23.8 \\
7.9 \\
8.5 \\
6.0 \\
7.7 \\
0.0\end{array}$ & $\begin{array}{r}6.6 \\
7.1 \\
4.7 \\
25.8 \\
20.4 \\
6.4 \\
9.0 \\
10.4 \\
5.5 \\
0.0\end{array}$ & $\begin{array}{r}6.8 \\
5.8 \\
3.0 \\
26.3 \\
24.0 \\
7.1 \\
9.0 \\
12.0 \\
0.9 \\
0.0\end{array}$ & $\begin{array}{r}4.4 \\
7.1 \\
3.0 \\
30.1 \\
20.0 \\
8.2 \\
7.7 \\
14.8 \\
0.7 \\
0.0\end{array}$ & $\begin{array}{r}4.7 \\
9.9 \\
3.6 \\
30.7 \\
12.3 \\
9.3 \\
6.6 \\
14.5 \\
8.5 \\
0.0\end{array}$ & $\begin{array}{r}6.6 \\
7.4 \\
3.9 \\
24.9 \\
5.8 \\
10.4 \\
9.0 \\
15.9 \\
16.2 \\
0.0\end{array}$ & $\begin{array}{l}3 \\
6 \\
3\end{array}$ \\
\hline
\end{tabular}


1969

39 Deareas North Latitude

\begin{tabular}{|c|c|c|c|c|c|c|c|c|c|c|c|c|c|c|c|c|}
\hline WPT & 126 & 122 & 118 & 114 & 110 & 106 & $\begin{array}{l}D 0 a \\
102\end{array}$ & $\begin{array}{c}98 \\
98\end{array}$ & $\begin{array}{l}\text { Lor } \\
9 a\end{array}$ & $\begin{array}{c}\text { itutede } \\
90\end{array}$ & 86 & 82 & $7 \mathrm{~B}$ & 74 & 70 & 66 \\
\hline $\begin{array}{r}1 \\
2 \\
3 \\
4 \\
5 \\
6 \\
7 \\
8 \\
9 \\
10\end{array}$ & $\begin{array}{r}1.9 \\
3.0 \\
2.7 \\
0.0 \\
0.5 \\
0.0 \\
7.1 \\
3.0 \\
81.6 \\
0.0\end{array}$ & $\begin{array}{r}3.0 \\
3.0 \\
1.9 \\
4.9 \\
9.6 \\
0.0 \\
6.8 \\
2.2 \\
68.5 \\
0.0\end{array}$ & $\begin{array}{r}1.6 \\
3.3 \\
2.5 \\
7.7 \\
21.4 \\
0.0 \\
5.8 \\
2.7 \\
55.1 \\
0.0\end{array}$ & $\begin{array}{r}1.9 \\
7.7 \\
3.3 \\
11.0 \\
30.7 \\
0.0 \\
7.1 \\
2.5 \\
40.8 \\
0.0\end{array}$ & $\begin{array}{r}1.4 \\
3.0 \\
1.9 \\
17.5 \\
30.4 \\
0.0 \\
13.4 \\
1.1 \\
31.2 \\
0.0\end{array}$ & $\begin{array}{r}1.6 \\
1.6 \\
2.2 \\
29.3 \\
19.4 \\
0.5 \\
20.8 \\
2.5 \\
23.0 \\
0.0\end{array}$ & $\begin{array}{r}2.5 \\
4.7 \\
2.7 \\
23.6 \\
25.8 \\
2.7 \\
19.2 \\
0.5 \\
18.4 \\
0.0\end{array}$ & $\begin{array}{r}4.7 \\
5.5 \\
3.0 \\
22.5 \\
26.5 \\
8.2 \\
14.5 \\
1.6 \\
13.2 \\
0.0\end{array}$ & $\begin{array}{r}3.8 \\
4.4 \\
9.4 \\
25.5 \\
25.5 \\
11.8 \\
19.0 \\
2.2 \\
8.5 \\
0.0\end{array}$ & $\begin{array}{r}4.7 \\
6.3 \\
4.9 \\
26.8 \\
23.0 \\
14.5 \\
12.1 \\
1.6 \\
6.0 \\
0.0\end{array}$ & $\begin{array}{r}5.8 \\
6.3 \\
3.0 \\
30.4 \\
20.3 \\
15.6 \\
11.0 \\
3.6 \\
4.1 \\
0.0\end{array}$ & $\begin{array}{r}6.6 \\
1.1 \\
3.0 \\
30.7 \\
20.0 \\
14.5 \\
9.6 \\
5.8 \\
5.6 \\
0.0\end{array}$ & $\begin{array}{r}5.5 \\
4.7 \\
4.4 \\
35.3 \\
14.5 \\
12.9 \\
9.0 \\
7.4 \\
6.3 \\
0.0\end{array}$ & $\begin{array}{r}5.8 \\
0.7 \\
3.0 \\
29.9 \\
4.7 \\
18.1 \\
11.8 \\
10.1 \\
12.1 \\
0.0\end{array}$ & $\begin{array}{r}5.2 \\
5.5 \\
3.0 \\
20.0 \\
1.6 \\
21.6 \\
6.6 \\
14.5 \\
20.3 \\
0.0\end{array}$ & $\begin{array}{r}0.0 \\
0.0 \\
0.0 \\
0.0 \\
0.0 \\
0.0 \\
0.0 \\
0.0 \\
0.0 \\
100.0\end{array}$ \\
\hline
\end{tabular}

36 Deprees North Lattude

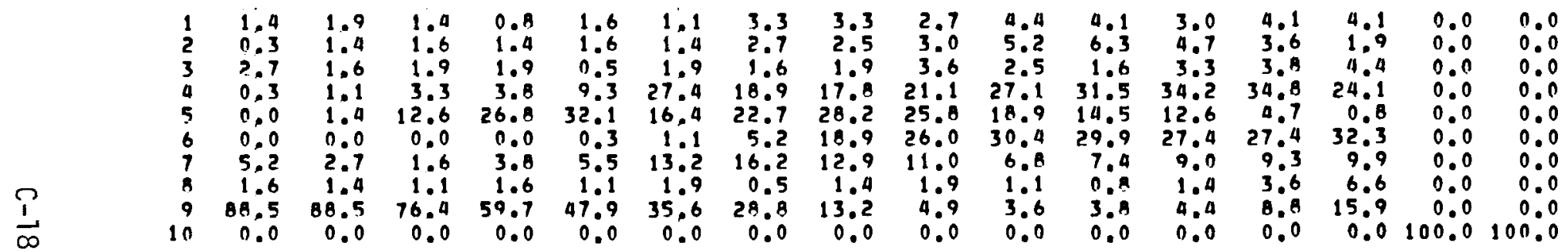

\section{Deorees North Lotftude}

\begin{tabular}{|c|c|c|c|c|c|c|c|c|c|c|c|c|c|c|c|c|}
\hline $\begin{array}{l}1 \\
2 \\
3 \\
4 \\
5 \\
6 \\
7 \\
8 \\
9 \\
10\end{array}$ & $\begin{array}{r}0.0 \\
0.0 \\
0.0 \\
0.0 \\
0.0 \\
0.0 \\
0.0 \\
0.0 \\
0.0 \\
100.0\end{array}$ & $\begin{array}{r}1.9 \\
0.0 \\
1.9 \\
0.0 \\
0.3 \\
0.0 \\
1.1 \\
0.3 \\
94.5 \\
0.0\end{array}$ & $\begin{array}{r}0.5 \\
0.3 \\
0.5 \\
1.1 \\
3.6 \\
0.0 \\
1.9 \\
0.3 \\
91.8 \\
0.0\end{array}$ & $\begin{array}{r}0.3 \\
0.0 \\
0.8 \\
1.4 \\
9.9 \\
0.0 \\
4.1 \\
0.8 \\
82.7 \\
0.0\end{array}$ & $\begin{array}{r}0.3 \\
0.5 \\
1.1 \\
5.2 \\
24.7 \\
0.0 \\
3.6 \\
0.5 \\
64.1 \\
0.0\end{array}$ & $\begin{array}{r}0.5 \\
0.5 \\
1.6 \\
17.5 \\
13.2 \\
1.4 \\
12.3 \\
0.8 \\
52.1 \\
0.0\end{array}$ & $\begin{array}{r}2.2 \\
1.4 \\
0.8 \\
9.3 \\
22.2 \\
14.2 \\
12.6 \\
0.8 \\
36.4 \\
0.0\end{array}$ & $\begin{array}{r}2.5 \\
1.6 \\
0.8 \\
13.4 \\
26.3 \\
31.2 \\
9.6 \\
0.5 \\
14.0 \\
0.0\end{array}$ & $\begin{array}{r}3.0 \\
2.5 \\
1.1 \\
17.5 \\
20.5 \\
39.6 \\
8.8 \\
1.6 \\
6.3 \\
0.0\end{array}$ & $\begin{array}{r}2.5 \\
4.1 \\
1.4 \\
24.9 \\
10.2 \\
42.2 \\
6.0 \\
0.5 \\
4.1 \\
0.0\end{array}$ & $\begin{array}{r}2.5 \\
3.6 \\
1.1 \\
27.4 \\
12.1 \\
41.1 \\
6.3 \\
0.8 \\
5.2 \\
0.0\end{array}$ & $\begin{array}{r}1.6 \\
3.3 \\
2.5 \\
30.1 \\
6.8 \\
38.9 \\
7.9 \\
1.1 \\
7.7 \\
0.0\end{array}$ & $\begin{array}{r}1.9 \\
1.6 \\
3.0 \\
26.3 \\
1.9 \\
43.6 \\
7.9 \\
1.9 \\
12 . \\
0.0\end{array}$ & $\begin{array}{r}2.5 \\
1.4 \\
2.7 \\
14.2 \\
0.0 \\
43.3 \\
7.9 \\
4.1 \\
23.0 \\
0.0\end{array}$ & $\begin{array}{r}0.0 \\
0.0 \\
0.0 \\
0.0 \\
0.0 \\
0.0 \\
0.0 \\
0.0 \\
0.0 \\
100.0\end{array}$ & $\begin{array}{r}0.0 \\
0.0 \\
0.0 \\
0.0 \\
0.0 \\
0.0 \\
0.0 \\
0.0 \\
0.0 \\
100.0\end{array}$ \\
\hline
\end{tabular}

30 Degrees North Lattude

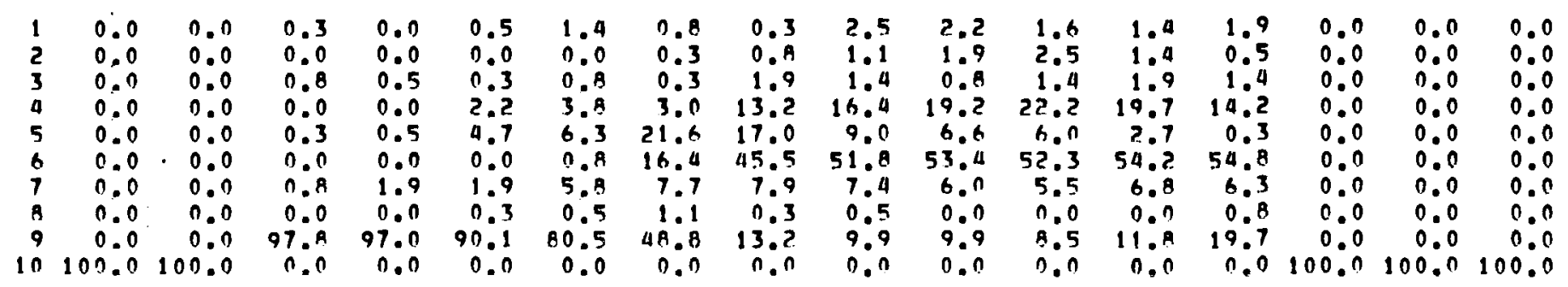


1969

27 Dearees North Lotltude

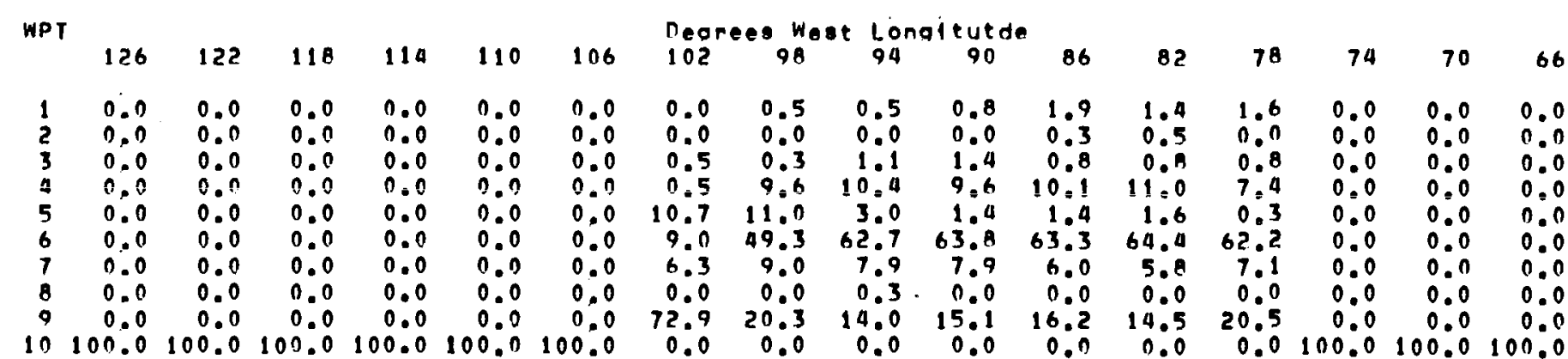

24 Degrees North Letltuda

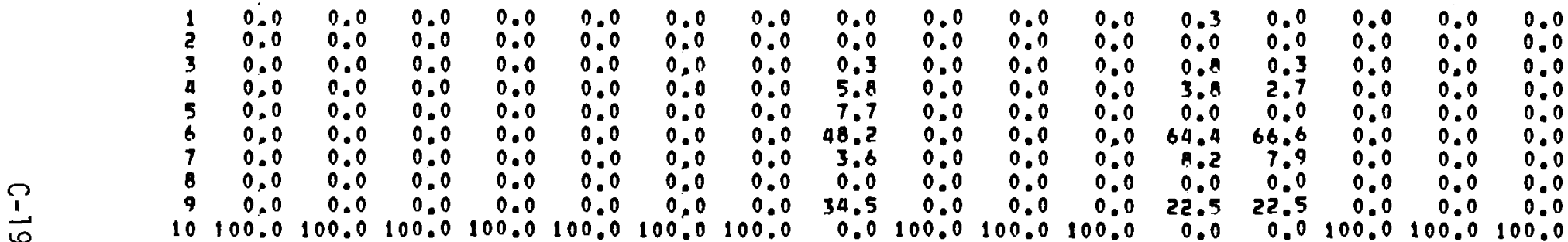


1970

51 Dagrees North Lattude

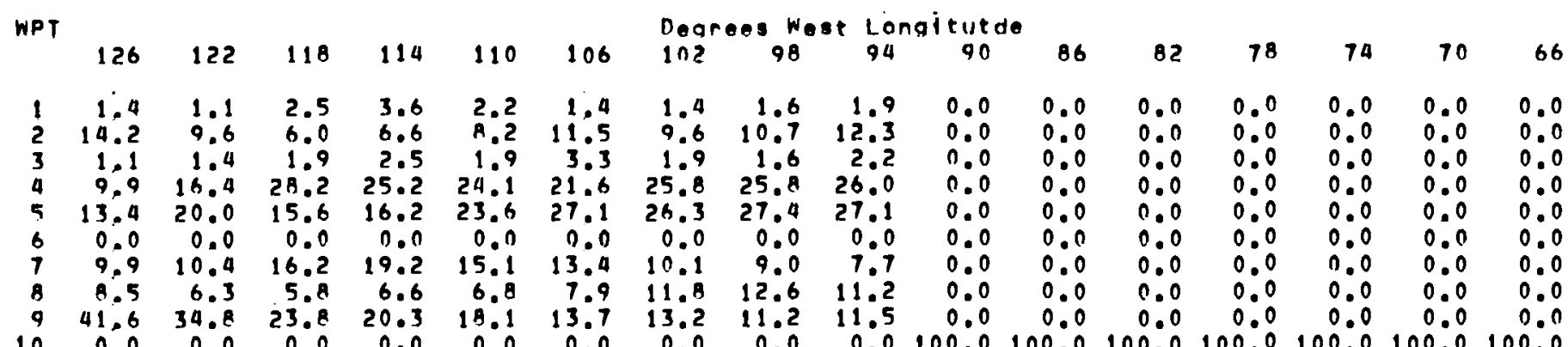

48 Degrees North Lot Itude

\begin{tabular}{|c|c|c|c|c|c|c|c|c|c|c|c|c|c|c|c|c|}
\hline $\begin{array}{l}1 \\
2 \\
3 \\
4 \\
5 \\
6 \\
7 \\
8 \\
9 \\
10\end{array}$ & $\begin{array}{r}2.7 \\
7.9 \\
1.6 \\
3.8 \\
6.6 \\
0.0 \\
7.0 \\
10.1 \\
59.7 \\
0.0\end{array}$ & $\begin{array}{r}1.1 \\
11.0 \\
1.9 \\
13.2 \\
18.6 \\
0.0 \\
7.4 \\
6.3 \\
40.5 \\
0.0\end{array}$ & $\begin{array}{r}1.9 \\
5.2 \\
1.6 \\
18.9 \\
22.5 \\
0.0 \\
12.3 \\
5.5 \\
32.1 \\
0.0\end{array}$ & $\begin{array}{r}3.6 \\
3.8 \\
2.2 \\
29.9 \\
17.0 \\
0.0 \\
17.5 \\
4.9 \\
21.1 \\
0.0\end{array}$ & $\begin{array}{r}3.0 \\
7.1 \\
3.8 \\
27.1 \\
16.7 \\
0.0 \\
20.5 \\
5.2 \\
16.4 \\
0.0\end{array}$ & $\begin{array}{r}2.7 \\
7.4 \\
3.8 \\
24.4 \\
23.3 \\
0.0 \\
15.9 \\
6.0 \\
16.4 \\
0.0\end{array}$ & $\begin{array}{r}3.3 \\
7.7 \\
3.3 \\
23.0 \\
23.8 \\
0.0 \\
13.2 \\
9.6 \\
16.2 \\
0.0\end{array}$ & $\begin{array}{r}3.3 \\
10.1 \\
3.6 \\
26.0 \\
27.9 \\
0.0 \\
10.1 \\
9.9 \\
9.0 \\
0.0\end{array}$ & $\begin{array}{r}3.8 \\
13.7 \\
3.0 \\
26.6 \\
24.9 \\
0.0 \\
10.1 \\
9.0 \\
1.8 \\
0.0\end{array}$ & $\begin{array}{r}3.3 \\
16.2 \\
4.1 \\
26.8 \\
21.4 \\
0.0 \\
7.9 \\
10.1 \\
10.1 \\
0.0\end{array}$ & $\begin{array}{r}4.9 \\
13.4 \\
2.5 \\
26.8 \\
23.8 \\
0.3 \\
6.6 \\
11.5 \\
10.1 \\
0.0\end{array}$ & $\begin{array}{r}3.8 \\
12.9 \\
2.2 \\
26.3 \\
26.3 \\
1.1 \\
5.8 \\
12.6 \\
9.0 \\
0.0\end{array}$ & $\begin{array}{r}3.8 \\
12.3 \\
1.6 \\
26.8 \\
23.3 \\
1.6 \\
6.0 \\
13.2 \\
11.2 \\
0.0\end{array}$ & $\begin{array}{r}3.6 \\
7.9 \\
2.2 \\
27.4 \\
20.8 \\
2.2 \\
5.5 \\
18.1 \\
12.3 \\
0.0\end{array}$ & $\begin{array}{r}3.0 \\
9.3 \\
1.9 \\
26.0 \\
14.5 \\
2.5 \\
6.6 \\
21.9 \\
14.2 \\
0.0\end{array}$ & $\begin{array}{r}2.2 \\
11.0 \\
2.5 \\
22.2 \\
11.0 \\
3.3 \\
6.0 \\
20.5 \\
21.04 \\
0.0\end{array}$ \\
\hline
\end{tabular}

45 Degrees North Latitude

\begin{tabular}{|c|c|c|c|c|c|c|c|c|c|c|c|c|c|c|c|c|}
\hline $\begin{array}{l}1 \\
2 \\
3 \\
0 \\
5 \\
6 \\
7 \\
8 \\
9 \\
10\end{array}$ & $\begin{array}{r}1.1 \\
6.6 \\
1.6 \\
1.9 \\
2.7 \\
0.0 \\
4.7 \\
0.5 \\
72.9 \\
0.0\end{array}$ & $\begin{array}{r}1.6 \\
7.7 \\
3.8 \\
11.2 \\
19.7 \\
0.0 \\
6.0 \\
4.1 \\
45.8 \\
0.0\end{array}$ & $\begin{array}{r}1.6 \\
6.3 \\
2.5 \\
15.6 \\
26.0 \\
0.0 \\
8.5 \\
3.6 \\
35.0 \\
0.0\end{array}$ & $\begin{array}{r}1.9 \\
3.8 \\
3.3 \\
23.3 \\
26.3 \\
0.0 \\
15.6 \\
1.9 \\
23.8 \\
0.0\end{array}$ & $\begin{array}{r}4.0 \\
0.7 \\
1.9 \\
31.2 \\
16.4 \\
0.0 \\
20.3 \\
2.2 \\
18.9 \\
0.0\end{array}$ & $\begin{array}{r}4.9 \\
6.6 \\
2.2 \\
24.7 \\
17.3 \\
0.0 \\
22.2 \\
4.7 \\
17.5 \\
0.0\end{array}$ & $\begin{array}{r}0.0 \\
7.1 \\
3.3 \\
23.6 \\
23.0 \\
0.0 \\
17.3 \\
5.8 \\
15.6 \\
0.0\end{array}$ & $\begin{array}{r}0.4 \\
8.5 \\
3.3 \\
28.5 \\
26.3 \\
0.0 \\
11.2 \\
6.8 \\
11.0 \\
0.0\end{array}$ & $\begin{array}{r}5.2 \\
11.2 \\
4.4 \\
27.7 \\
26.6 \\
0.3 \\
10.4 \\
5.8 \\
3.5 \\
0.0\end{array}$ & $\begin{array}{r}6.0 \\
11.2 \\
3.8 \\
29.6 \\
23.6 \\
1.4 \\
9.6 \\
7.9 \\
6.8 \\
0.0\end{array}$ & $\begin{array}{r}5.8 \\
12.6 \\
6.3 \\
20.3 \\
26.8 \\
1.9 \\
8.8 \\
9.9 \\
7.7 \\
0.0\end{array}$ & $\begin{array}{r}4.7 \\
11.5 \\
4.7 \\
23.6 \\
24.1 \\
1.9 \\
9.9 \\
10.9 \\
9.3 \\
0.0\end{array}$ & $\begin{array}{r}3.3 \\
11.2 \\
4.1 \\
20.1 \\
25.2 \\
2.5 \\
6.3 \\
12.6 \\
10.7 \\
0.0\end{array}$ & $\begin{array}{r}3.3 \\
9.0 \\
3.6 \\
27.4 \\
19.7 \\
4.1 \\
7.1 \\
14.5 \\
11.2 \\
0.0\end{array}$ & $\begin{array}{r}3.0 \\
11.2 \\
2.2 \\
29.0 \\
11.2 \\
4.9 \\
4.4 \\
16.7 \\
17.3 \\
0.0\end{array}$ & $\begin{array}{r}3.8 \\
10.7 \\
1.9 \\
22.7\end{array}$ \\
\hline
\end{tabular}

42 Dearees North Lattude

\begin{tabular}{|c|c|c|c|c|c|c|c|c|c|c|c|c|c|c|c|c|}
\hline $\begin{array}{l}1 \\
2 \\
3 \\
4 \\
5 \\
6 \\
7 \\
8 \\
9 \\
0\end{array}$ & $\begin{array}{r}1.9 \\
4.4 \\
3.3 \\
1.4 \\
1.0 \\
0.0 \\
3.0 \\
4.7 \\
80.0 \\
0.0\end{array}$ & $\begin{array}{r}1.9 \\
3.0 \\
3.8 \\
8.5 \\
19.7 \\
0.0 \\
7.9 \\
3.6 \\
51.5 \\
0.0\end{array}$ & $\begin{array}{r}1.1 \\
4.9 \\
2.5 \\
12.6 \\
28.9 \\
0.0 \\
10.7 \\
2.8 \\
37.3 \\
0.0\end{array}$ & $\begin{array}{r}1.4 \\
3.8 \\
1.9 \\
19.7 \\
28.5 \\
0.0 \\
10.1 \\
2.7 \\
31.8 \\
0.0\end{array}$ & $\begin{array}{r}2.2 \\
5.2 \\
1.6 \\
28.2 \\
21.9 \\
0.0 \\
13.2 \\
1.0 \\
26.3 \\
0.0\end{array}$ & $\begin{array}{r}3.3 \\
3.8 \\
1.6 \\
29.3 \\
16.2 \\
0.0 \\
22.2 \\
1.6 \\
21.9 \\
0.0\end{array}$ & $\begin{array}{r}2.5 \\
4.7 \\
2.5 \\
24.4 \\
22.2 \\
0.3 \\
19.7 \\
2.5 \\
21.4 \\
0.0\end{array}$ & $\begin{array}{r}4.1 \\
4.4 \\
2.7 \\
28.5 \\
27.7 \\
0.3 \\
14.8 \\
2.7 \\
14.8 \\
0.0\end{array}$ & $\begin{array}{r}7.4 \\
6.3 \\
3.8 \\
28.8 \\
28.2 \\
1.1 \\
12.9 \\
3.6 \\
7.9 \\
0.0\end{array}$ & $\begin{array}{r}6.3 \\
7.1 \\
4.7 \\
27.1 \\
28.8 \\
4.1 \\
9.9 \\
6.6 \\
5.5 \\
0.0\end{array}$ & $\begin{array}{r}6.8 \\
7.9 \\
5.5 \\
18.9 \\
28.9 \\
5.2 \\
10.7 \\
9.6 \\
6.6 \\
0.0\end{array}$ & $\begin{array}{r}7.1 \\
10.1 \\
3.0 \\
21.6 \\
20.8 \\
6.3 \\
10.7 \\
9.9 \\
10.4 \\
0.0\end{array}$ & $\begin{array}{r}5.2 \\
11.0 \\
3.8 \\
24.7 \\
20.5 \\
6.3 \\
9.9 \\
7.7 \\
11.0 \\
0.0\end{array}$ & $\begin{array}{r}3.0 \\
8.5 \\
4.7 \\
30.4 \\
14.2 \\
8.5 \\
8.5 \\
9.0 \\
13.2 \\
0.0\end{array}$ & $\begin{array}{r}3.6 \\
7.4 \\
5.0 \\
26.0 \\
5.5 \\
11.0 \\
6.0 \\
12.1 \\
22.7 \\
0.0\end{array}$ & $\begin{array}{r}4.1 \\
5.5 \\
1.6 \\
16.7 \\
2.7 \\
11.8 \\
5.5 \\
16.7 \\
35.1 \\
0.0\end{array}$ \\
\hline
\end{tabular}


1970

39 Deprees North Letltude

\begin{tabular}{|c|c|c|c|c|c|c|c|c|c|c|c|c|c|c|c|c|c|}
\hline WPT & 126 & 122 & 118 & 114 & 110 & 106 & $\begin{array}{l}\text { Degr } \\
102\end{array}$ & 9 & & ne & $\begin{array}{l}\text { tutde } \\
90\end{array}$ & 86 & 82 & 78 & 74 & 70 & 6 \\
\hline $\begin{array}{l}1 \\
2 \\
3 \\
4 \\
5 \\
6 \\
7 \\
6 \\
9 \\
10\end{array}$ & $\begin{array}{r}3.3 \\
1.04 \\
3.3 \\
0.5 \\
0.5 \\
0.0 \\
5.8 \\
3.6 \\
81.6 \\
0.0\end{array}$ & $\begin{array}{r}0.5 \\
1.6 \\
2.7 \\
0.1 \\
13.2 \\
0.0 \\
6.3 \\
2.7 \\
68.8 \\
0.0\end{array}$ & $\begin{array}{r}0.3 \\
3.0 \\
1.9 \\
6.2 \\
28.8 \\
0.0 \\
7.4 \\
1.1 \\
49.3 \\
0.0\end{array}$ & $\begin{array}{r}0.8 \\
3.6 \\
1.6 \\
14.0 \\
33.2 \\
0.0 \\
7.9 \\
1.1 \\
37.8 \\
0.0\end{array}$ & $\begin{array}{r}2.2 \\
2.2 \\
1.1 \\
21.1 \\
27.1 \\
0.0 \\
9.6 \\
0.0 \\
35.9 \\
0.0\end{array}$ & $\begin{array}{r}2.5 \\
1.1 \\
32.3 \\
16.4 \\
0.0 \\
15.6 \\
1.1 \\
25.8 \\
9.0\end{array}$ & $\begin{array}{r}2.7 \\
2.7 \\
2.7 \\
21.4 \\
21.1 \\
0.5 \\
23.0 \\
1.1 \\
24.7 \\
0.0\end{array}$ & $\begin{array}{r}4.7 \\
2.7 \\
5.5 \\
21.4 \\
27.7 \\
2.2 \\
19.2 \\
1.6 \\
15.1 \\
0.0\end{array}$ & $\begin{array}{r}4 \\
5 \\
3 \\
23 \\
27 \\
10 \\
15 \\
2 \\
6 \\
0\end{array}$ & $\begin{array}{l}.3 \\
8 \\
0 \\
9 \\
1 \\
.6 \\
.5 \\
.6 \\
.0\end{array}$ & $\begin{array}{r}4.7 \\
4.9 \\
4.9 \\
22.7 \\
26.0 \\
14.5 \\
14.2 \\
3.8 \\
4.1 \\
0.0\end{array}$ & $\begin{array}{r}6.3 \\
4.9 \\
4.1 \\
22.2 \\
25.2 \\
15.3 \\
11.2 \\
5.2 \\
5.5 \\
0.0\end{array}$ & $\begin{array}{r}6.8 \\
7.1 \\
5.2 \\
23.8 \\
19.7 \\
15.6 \\
9.3 \\
5.5 \\
6.8 \\
0.0\end{array}$ & $\begin{array}{r}3.8 \\
6.2 \\
3.3 \\
29.6 \\
15.3 \\
15.6 \\
8.5 \\
4.7 \\
11.0 \\
0.0\end{array}$ & $\begin{array}{r}4.4 \\
4.9 \\
3.0 \\
30.1 \\
6.0 \\
17.5 \\
7.9 \\
5.9 \\
20.3 \\
0.0\end{array}$ & $\begin{array}{r}3.8 \\
4.1 \\
1.1 \\
20.3 \\
2.3 \\
17.8 \\
6.8 \\
10.4 \\
30.4 \\
0.0\end{array}$ & $\begin{array}{ll}0.0 \\
0.0\end{array}$ \\
\hline
\end{tabular}

36 Dearees North Lotltude

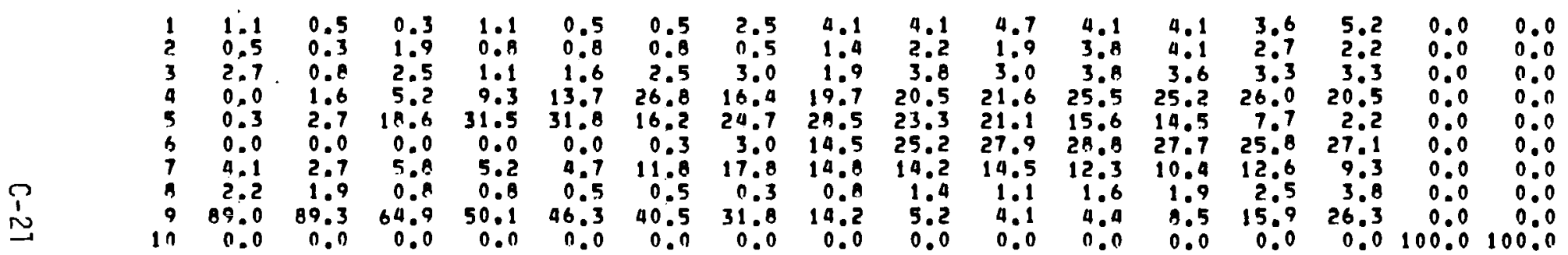

33 Degrees North Letitude

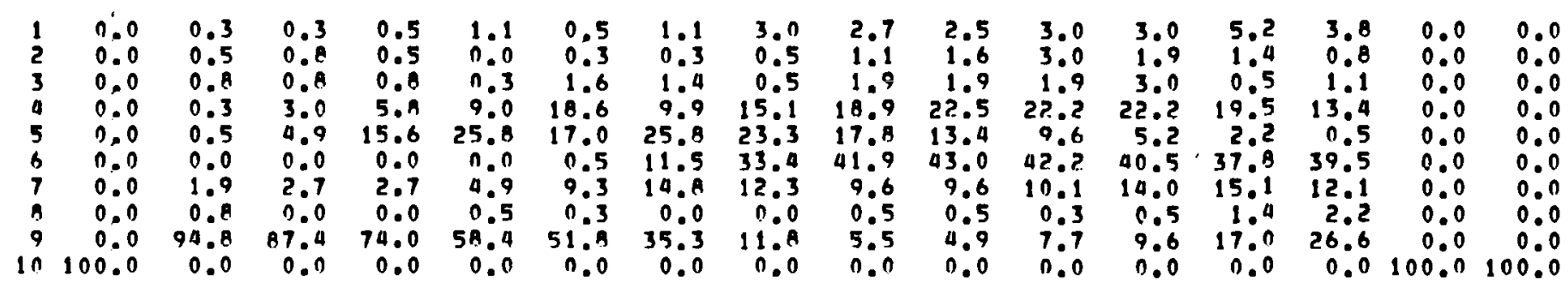

30 Deprees North Letltude

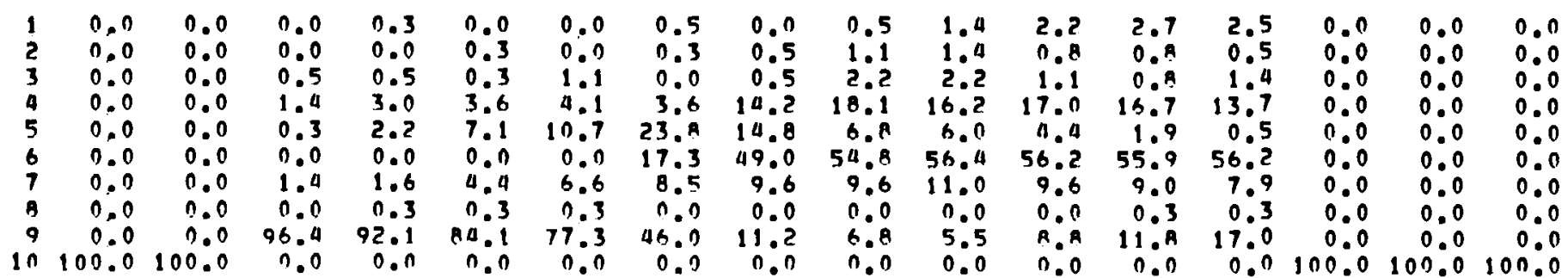


1970

27 Dearees North Lotitude

\begin{tabular}{|c|c|c|c|c|c|c|c|c|c|c|c|c|c|c|c|c|}
\hline WPT & 126 & 122 & 118 & 114 & 110 & 106 & $\begin{array}{l}\text { Deor } \\
102\end{array}$ & ${ }_{9 B}^{W}$ & $\begin{array}{l}\text { t Lor } \\
94\end{array}$ & $\begin{array}{l}\text { tut de } \\
90\end{array}$ & 86 & B2 & 78 & 74 & 70 & 66 \\
\hline $\begin{array}{r}1 \\
2 \\
3 \\
4 \\
5 \\
6 \\
7 \\
3 \\
9 \\
10\end{array}$ & $\begin{array}{r}n .0 \\
n .0 \\
0.0 \\
0.0 \\
0.0 \\
0.0 \\
0.0 \\
0.0 \\
0.0 \\
100.0\end{array}$ & $\begin{array}{r}0.0 \\
0.0 \\
0.0 \\
0.0 \\
0.0 \\
0.0 \\
0.0 \\
0.0 \\
0.0 \\
100.0\end{array}$ & $\begin{array}{r}0.0 \\
0.0 \\
0.0 \\
0.0 \\
0.0 \\
0.0 \\
0.0 \\
0.0 \\
0.0 \\
100.0\end{array}$ & $\begin{array}{r}0.0 \\
0.0 \\
0.0 \\
0.0 \\
0.0 \\
0.0 \\
0.0 \\
0.0 \\
0.0 \\
100.0\end{array}$ & $\begin{array}{r}0.0 \\
0.0 \\
0.0 \\
0.0 \\
0.0 \\
0.0 \\
0.0 \\
0.0 \\
0.0 \\
100.0\end{array}$ & $\begin{array}{r}0.0 \\
0.0 \\
0.0 \\
0.0 \\
0.0 \\
0.0 \\
0.0 \\
0.0 \\
0.0 \\
100.0\end{array}$ & $\begin{array}{r}0.0 \\
0.3 \\
0.0 \\
1.1 \\
13.2 \\
13.7 \\
5.8 \\
0.0 \\
66.0 \\
0.0\end{array}$ & $\begin{array}{r}0.8 \\
0.3 \\
0.3 \\
12.6 \\
7.9 \\
54.2 \\
7.9 \\
0.0 \\
15.9 \\
0.0\end{array}$ & $\begin{array}{r}0.8 \\
0.3 \\
0.5 \\
13.2 \\
2.7 \\
60.8 \\
9.3 \\
0.0 \\
12.3 \\
0.0\end{array}$ & $\begin{array}{r}0.5 \\
0.5 \\
1.6 \\
10.7 \\
2.7 \\
61.9 \\
9.0 \\
0.0 \\
12.9 \\
0.0\end{array}$ & $\begin{array}{r}0.8 \\
0.3 \\
0.5 \\
11.8 \\
0.5 \\
65.2 \\
7.1 \\
0.0 \\
13.7 \\
0.0\end{array}$ & $\begin{array}{r}1.4 \\
0.0 \\
0.5 \\
11.0 \\
0.3 \\
66.0 \\
5.2 \\
0.0 \\
15.6 \\
0.0\end{array}$ & $\begin{array}{r}0.8 \\
0.3 \\
1.1 \\
7.4 \\
0.0 \\
66.3 \\
6.3 \\
0.3 \\
17.5 \\
0.0\end{array}$ & $\begin{array}{r}0.0 \\
0.0 \\
0.0 \\
0.0 \\
0.0 \\
0.0 \\
0.0 \\
0.0 \\
0.0 \\
100.0\end{array}$ & $\begin{array}{r}0.0 \\
0.0 \\
0.0 \\
0.0 \\
0.0 \\
0.0 \\
0.0 \\
0.0 \\
0.0 \\
100.0\end{array}$ & $\begin{array}{r}0.0 \\
0.0 \\
0.0 \\
0.0 \\
0.0 \\
0.0 \\
0.0 \\
0.0 \\
0.0 \\
100.0\end{array}$ \\
\hline
\end{tabular}

24 Degrees North Lotitude

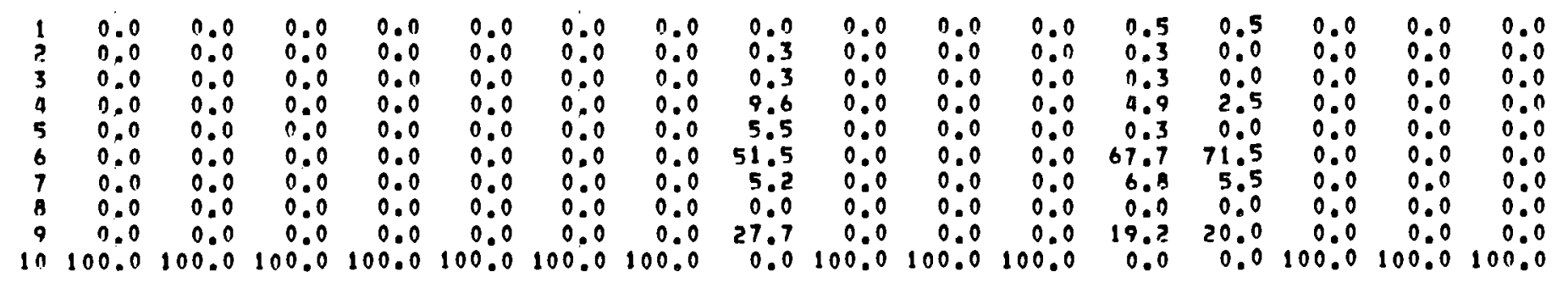


1971

51 Degrees North Lattude

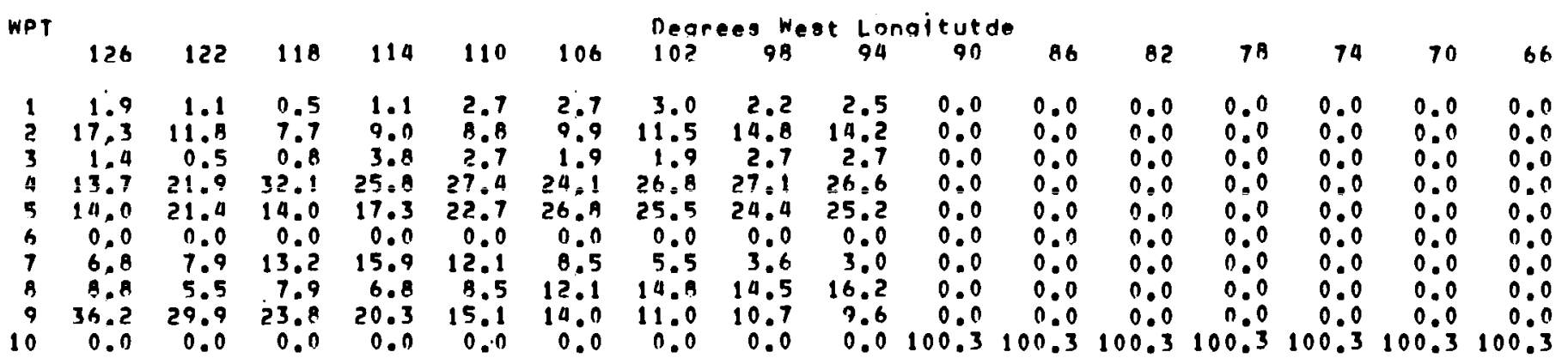

48 Degrees North Lat 1 tude

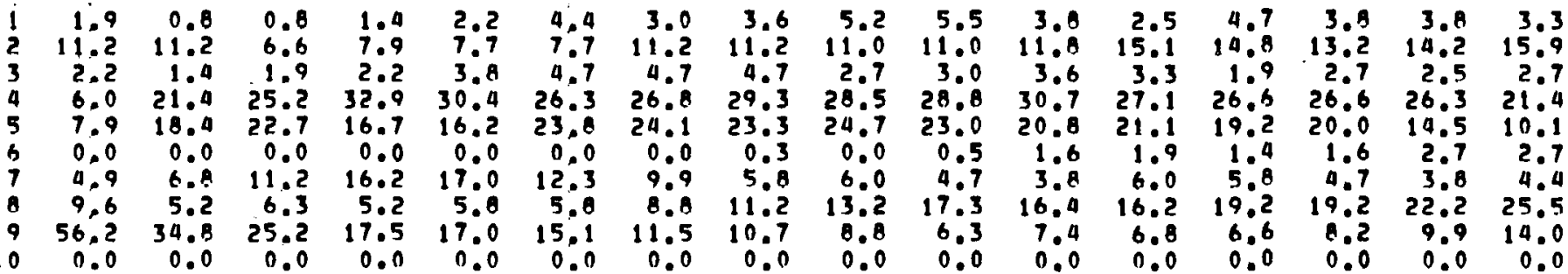

45 negrees North Liettude

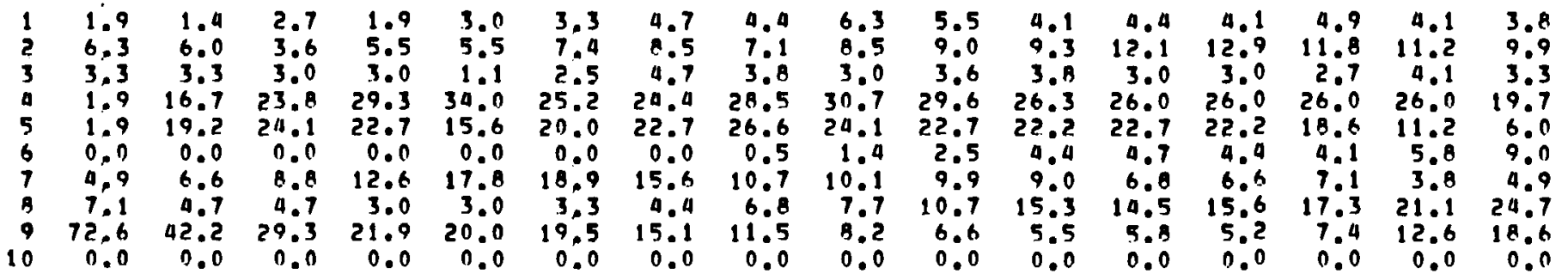

42 Deprans North Lot Itude

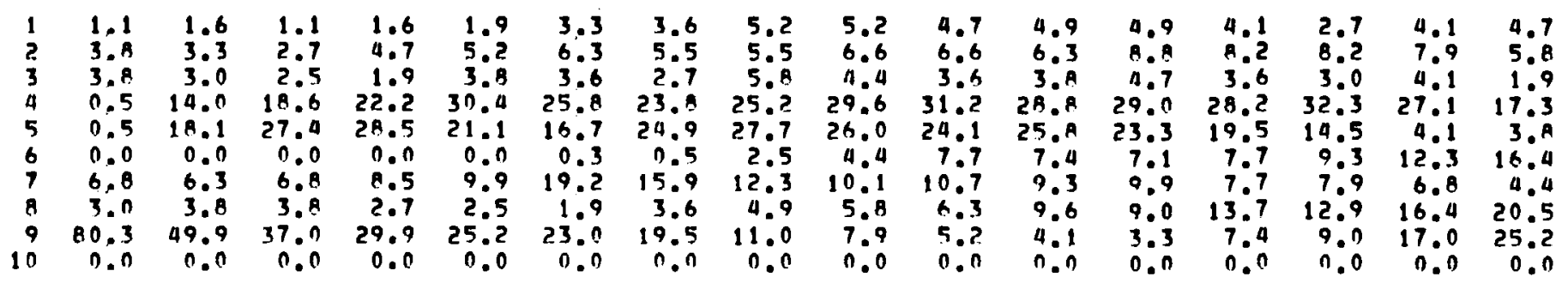


1971

39 Dearees North Letteude

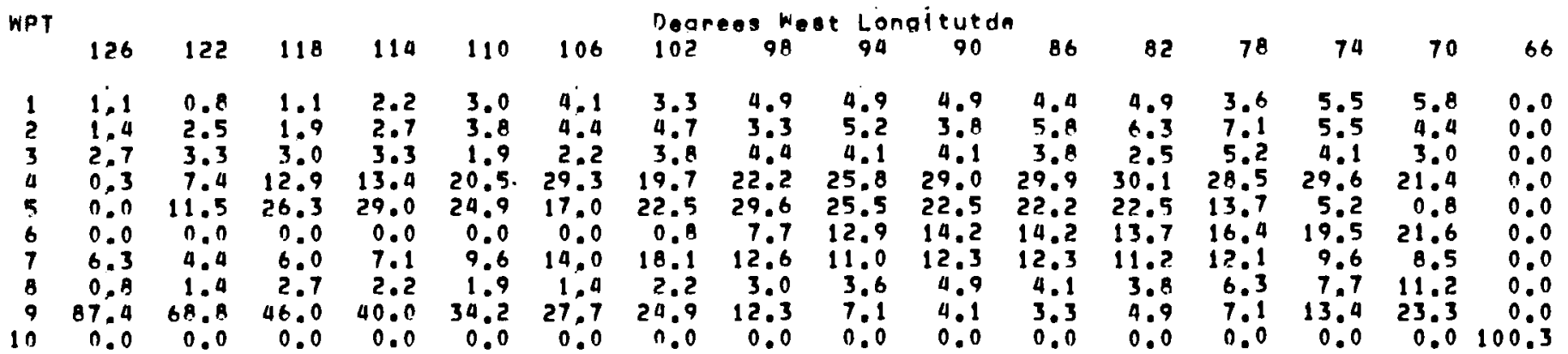

36 Deprees North Lattiude

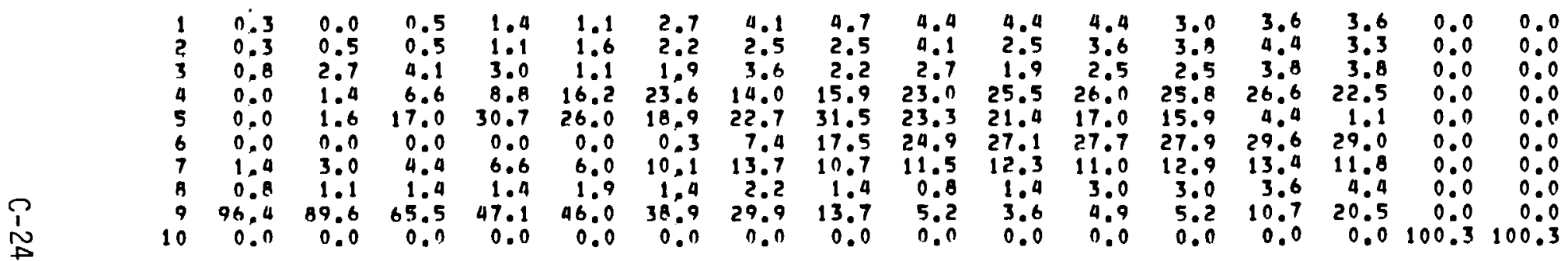

33 Degrees North Latltude

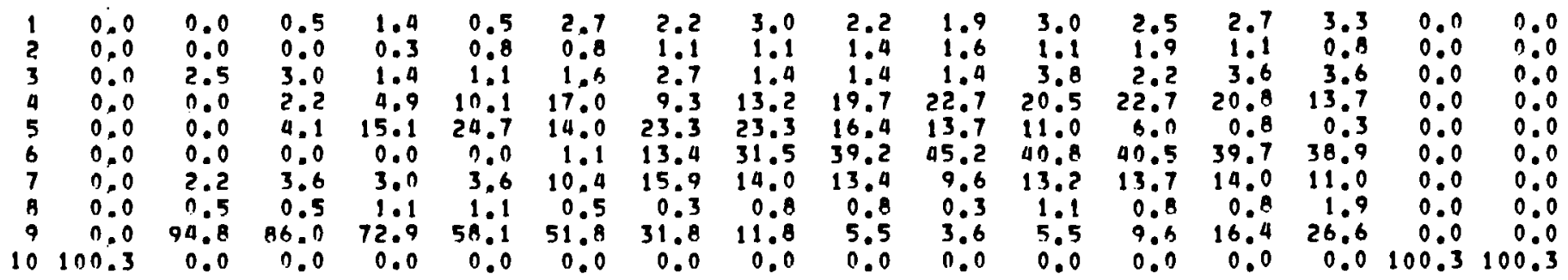

30 Dearees North Lotitude

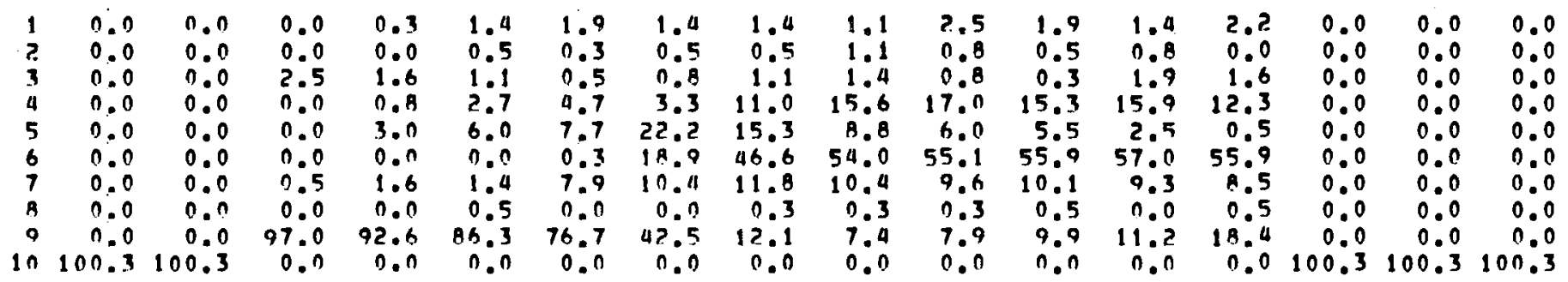


1971

27 Dearees North Lat tude

\begin{tabular}{|c|c|c|c|c|c|c|c|c|c|c|c|c|c|c|c|c|}
\hline WPT & 126 & 122 & 118 & 114 & 10 & 106 & $\begin{array}{l}\text { Dear } \\
102\end{array}$ & $\begin{array}{r}W \\
98\end{array}$ & & $\begin{array}{l}\text { Lor } \\
94\end{array}$ & $\begin{array}{c}\text { tut do } \\
90\end{array}$ & 86 & 82 & 78 & 74 & 70 \\
\hline $\begin{array}{l}1 \\
2 \\
3 \\
4 \\
5 \\
6 \\
7 \\
8 \\
9 \\
10\end{array}$ & $\begin{array}{r}0.0 \\
0.0 \\
0.0 \\
0.0 \\
0.0 \\
0.0 \\
0.0 \\
0.0 \\
0.0 \\
00.3\end{array}$ & $\begin{array}{l}0.0 \\
0.0 \\
0.0 \\
0.0 \\
0.0 \\
0.0 \\
0.0 \\
0.0 \\
00.0\end{array}$ & $\begin{array}{r}0.0 \\
0.0 \\
0.0 \\
0.0 \\
0.0 \\
0.0 \\
0.0 \\
0.0 \\
0.0 \\
00.3\end{array}$ & $\begin{array}{l}0.0 \\
0.0 \\
0.0 \\
0.0 \\
0.0 \\
0.0 \\
0.0 \\
0.0 \\
00.3\end{array}$ & $\begin{array}{l}0.0 \\
0.0 \\
0.0 \\
0.0 \\
0.0 \\
0.0 \\
0.0 \\
0.0\end{array}$ & 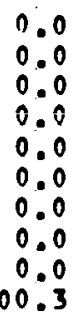 & $\begin{array}{r}0.0 \\
0.0 \\
0.3 \\
0.5 \\
8.2 \\
14.2 \\
0.2 \\
0.0 \\
68.5 \\
0.0\end{array}$ & $\begin{array}{r}0.3 \\
0.0 \\
0.3 \\
9.0 \\
8.2 \\
58.9 \\
9.3 \\
0.3 \\
13.7 \\
0.0\end{array}$ & & $\begin{array}{l}1.1 \\
0.3 \\
0.5 \\
0.8 \\
2.7 \\
5.5 \\
0.1 \\
0.0 \\
1.0 \\
0.0\end{array}$ & $\begin{array}{r}0.3 \\
0.5 \\
0.0 \\
7.7 \\
1.4 \\
69.9 \\
7.7 \\
0.3 \\
12.3 \\
0.0\end{array}$ & $\begin{array}{r}0.8 \\
0.0 \\
0.0 \\
7.4 \\
1.6 \\
71.5 \\
7.9 \\
0.0 \\
10.7 \\
0.0\end{array}$ & $\begin{array}{r}0.0 \\
0.3 \\
0.8 \\
0.8 \\
0.5 \\
71.8 \\
6.8 \\
0.0 \\
11.5 \\
0.0\end{array}$ & $\begin{array}{r}0.3 \\
0.0 \\
0.3 \\
5.0 \\
0.0 \\
69.9 \\
6.6 \\
0.0 \\
17.3 \\
0.0\end{array}$ & $\begin{array}{l}0 \\
0 \\
0 \\
0 \\
0 \\
0 \\
0 \\
0 \\
0 \\
0\end{array}$ & $\begin{array}{r}0.0 \\
0.0 \\
0.0 \\
0.0 \\
0.0 \\
0.0 \\
0.0 \\
0.0 \\
0.0 \\
0.0 .3\end{array}$ \\
\hline
\end{tabular}

24 Deprees North Latitude

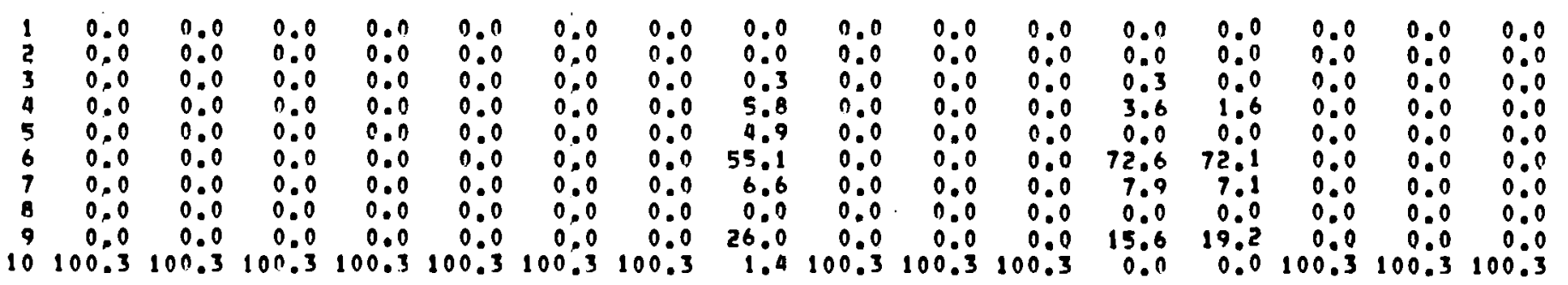


1972

51 Degrees North Latitude

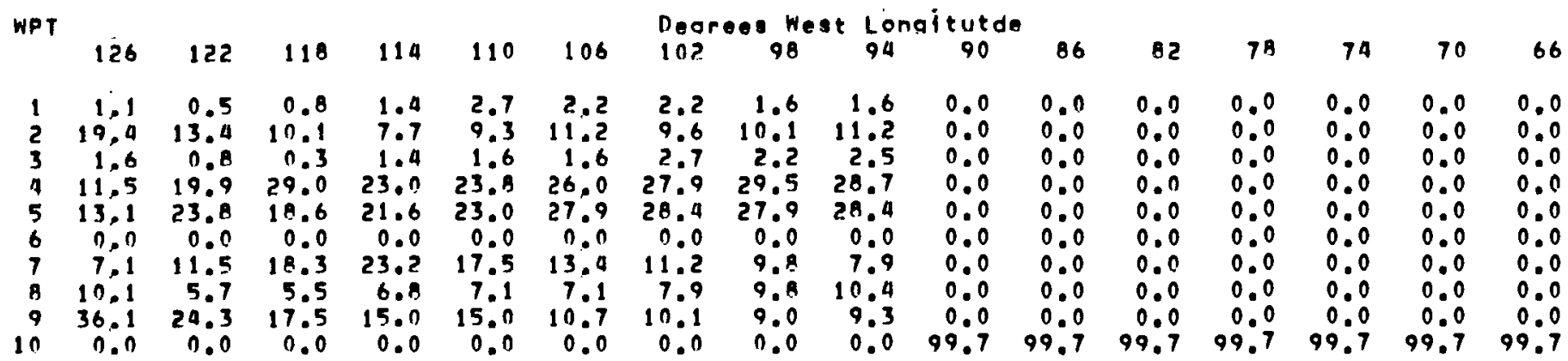

\section{aB Dearees North Latitude}

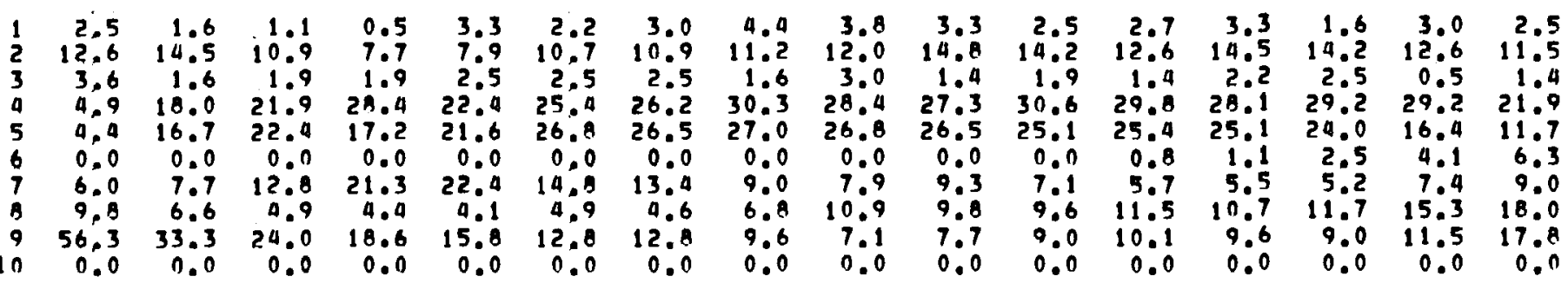

a5 Degrees North Lattude

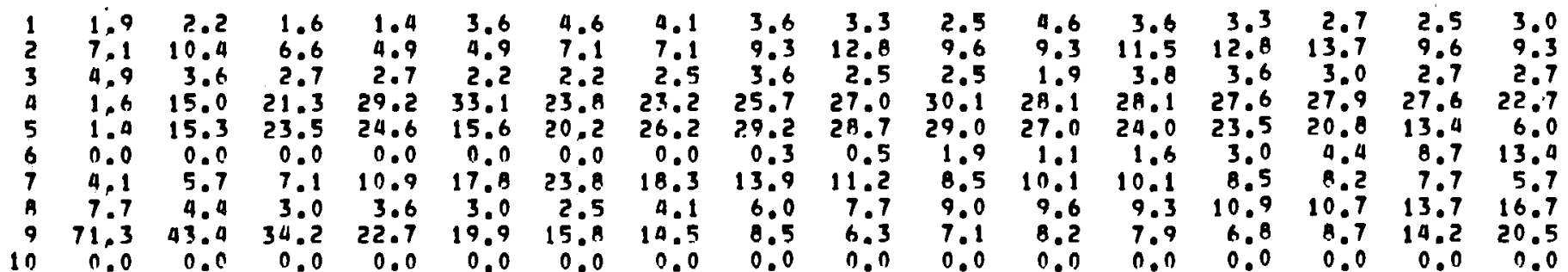

42 Dearees North Letitude

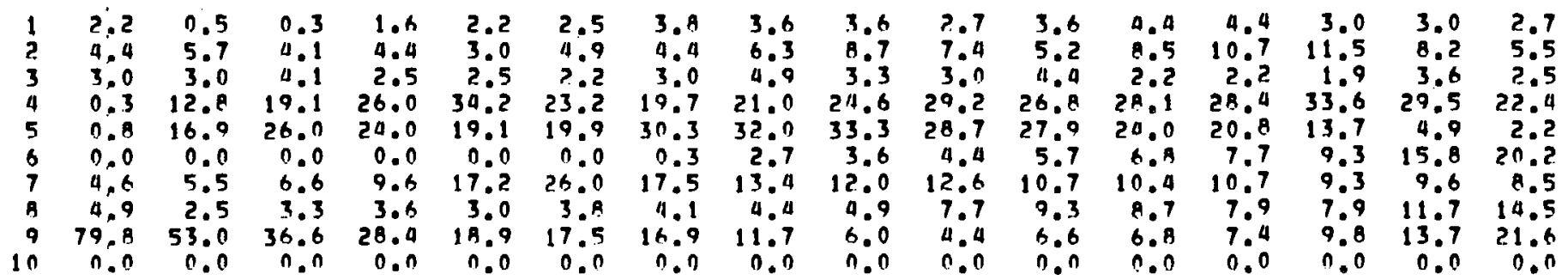


1972

39 Degreos North Letitude

\begin{tabular}{|c|c|c|c|c|c|c|c|c|c|c|c|c|c|c|c|c|}
\hline WP T & 126 & 122 & 118 & 114 & 110 & 106 & $\begin{array}{l}\text { Dear } \\
102\end{array}$ & a ${ }_{98}^{W e a t}$ & Lon & $\begin{array}{c}\text { tut de } \\
90\end{array}$ & 86 & B2 & 78 & 74 & 70 & 66 \\
\hline $\begin{array}{r}1 \\
2 \\
3 \\
6 \\
5 \\
6 \\
7 \\
8 \\
9 \\
10\end{array}$ & $\begin{array}{r}1.1 \\
1.9 \\
3.3 \\
0.0 \\
0.0 \\
0.0 \\
4.1 \\
3.8 \\
85.8 \\
0.0\end{array}$ & $\begin{array}{r}0.3 \\
1.1 \\
3.3 \\
7.7 \\
11.5 \\
0.0 \\
4.6 \\
2.5 \\
69.1 \\
0.0\end{array}$ & $\begin{array}{r}0.5 \\
2.5 \\
2.2 \\
15.5 \\
25.1 \\
0.0 \\
5.5 \\
3.3 \\
45.1 \\
0.0\end{array}$ & $\begin{array}{r}1.1 \\
3.3 \\
2.2 \\
20.2 \\
27.0 \\
0.0 \\
7.7 \\
3.0 \\
35.5 \\
0.0\end{array}$ & $\begin{array}{r}1.9 \\
2.5 \\
2.7 \\
25.1 \\
21.9 \\
0.0 \\
16.1 \\
3.0 \\
26.8 \\
0.0\end{array}$ & $\begin{array}{r}1.4 \\
4.6 \\
1.9 \\
24.6 \\
19.1 \\
0.3 \\
22.4 \\
2.7 \\
23.0 \\
0.0\end{array}$ & $\begin{array}{r}1.9 \\
4.1 \\
1.4 \\
16.4 \\
28.7 \\
1.4 \\
22.7 \\
2.5 \\
21.0 \\
0.0\end{array}$ & $\begin{array}{r}2.5 \\
6.6 \\
2.2 \\
10.6 \\
33.6 \\
4.9 \\
15.8 \\
3.6 \\
12.3 \\
0.0\end{array}$ & $\begin{array}{r}3.0 \\
4.9 \\
3.6 \\
24.0 \\
32.2 \\
9.0 \\
12.8 \\
3.8 \\
6.6 \\
0.0\end{array}$ & $\begin{array}{r}3.3 \\
5.2 \\
3.8 \\
28.4 \\
27.3 \\
10.4 \\
13.1 \\
4.4 \\
4.1 \\
0.0\end{array}$ & $\begin{array}{r}4.6 \\
5.2 \\
2.5 \\
26.0 \\
26.2 \\
11.7 \\
11.2 \\
6.6 \\
6.0 \\
0.0\end{array}$ & $\begin{array}{r}3.8 \\
7.9 \\
2.2 \\
27.0 \\
21.9 \\
13.1 \\
12.0 \\
6.3 \\
5.7 \\
0.0\end{array}$ & $\begin{array}{r}2.7 \\
6.5 \\
2.7 \\
30.1 \\
14.8 \\
16.1 \\
12.0 \\
5.2 \\
7.9 \\
0.0\end{array}$ & $\begin{array}{r}2.5 \\
7.7 \\
3.3 \\
33.3 \\
4.9 \\
18.9 \\
9.8 \\
5.5 \\
14.2 \\
0.0\end{array}$ & $\begin{array}{r}3.8 \\
6.0 \\
3.3 \\
24.0 \\
1.0 \\
23.0 \\
9.6 \\
7.7 \\
21.0 \\
0.0\end{array}$ & $\begin{array}{rl}0.0 & 0 \\
0.0 \\
0.0 \\
0.0 \\
0.0 \\
0.0 \\
0.0 \\
0.0 \\
0.0 \\
99.7\end{array}$ \\
\hline
\end{tabular}

36 Dearees North Lotitude

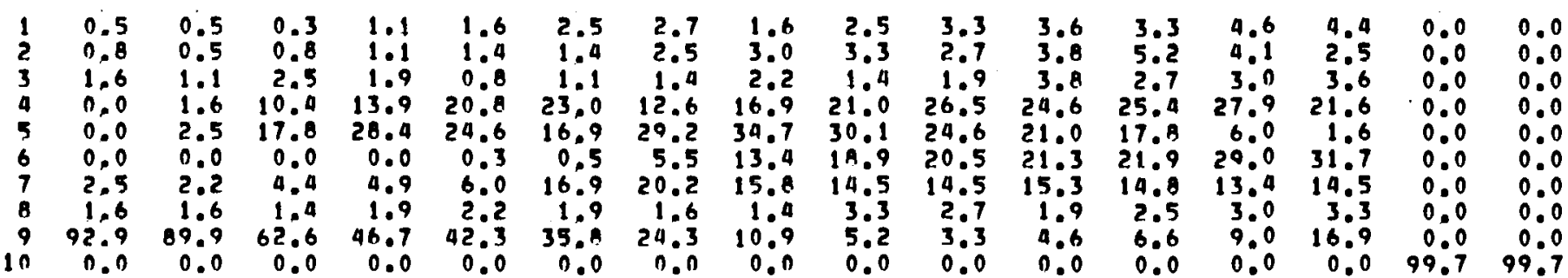

33 Dearees North Latltude

\begin{tabular}{|c|c|c|c|c|c|c|c|c|c|c|c|c|c|c|c|c|}
\hline $\begin{array}{l}1 \\
2 \\
3 \\
4 \\
5 \\
6 \\
7 \\
6 \\
9 \\
10\end{array}$ & $\begin{array}{r}0.0 \\
0.0 \\
0.0 \\
0.0 \\
0.0 \\
0.0 \\
0.0 \\
0.0 \\
0.0 \\
99.7\end{array}$ & $\begin{array}{r}0.3 \\
0.3 \\
1.1 \\
0.3 \\
0.0 \\
0.0 \\
1.6 \\
0.3 \\
96.2 \\
0.0\end{array}$ & $\begin{array}{r}0.3 \\
0.5 \\
1.1 \\
5.2 \\
6.0 \\
0.0 \\
2.5 \\
0.3 \\
0.2 \\
0.0\end{array}$ & $\begin{array}{r}0.5 \\
0.8 \\
1.1 \\
0.5 \\
10.4 \\
0.0 \\
2.5 \\
0.3 \\
76.0 \\
0.0\end{array}$ & $\begin{array}{r}1.4 \\
1.1 \\
0.8 \\
11.7 \\
23.5 \\
0.3 \\
4.9 \\
1.1 \\
55.2 \\
0.0\end{array}$ & $\begin{array}{r}1.4 \\
0.5 \\
0.5 \\
17.2 \\
16.1 \\
1.6 \\
14.2 \\
0.8 \\
47.5 \\
0.0\end{array}$ & $\begin{array}{r}1.6 \\
1.9 \\
1.6 \\
1.5 \\
27.3 \\
12.8 \\
15.6 \\
0.5 \\
30.1 \\
0.0\end{array}$ & $\begin{array}{r}1.4 \\
1.4 \\
0.8 \\
15.3 \\
27.9 \\
28.1 \\
15.6 \\
0.5 \\
9.0 \\
0.0\end{array}$ & $\begin{array}{r}1.6 \\
1.9 \\
1.1 \\
20.2 \\
20.5 \\
36.1 \\
12.3 \\
1.1 \\
5.2 \\
0.0\end{array}$ & $\begin{array}{r}1.1 \\
2.5 \\
1.6 \\
21.6 \\
18.0 \\
38.8 \\
10.1 \\
1.4 \\
4.9 \\
0.0\end{array}$ & $\begin{array}{r}3.0 \\
3.0 \\
1.9 \\
21.9 \\
14.8 \\
39.1 \\
10.4 \\
0.3\end{array}$ & $\begin{array}{r}2.7 \\
2.7 \\
2.5 \\
23.2 \\
8.2 \\
39.1 \\
12.6 \\
1.1 \\
7.9 \\
0.0\end{array}$ & $\begin{array}{r}2.7 \\
0.8 \\
3.8 \\
20.2 \\
1.9 \\
45.1 \\
12.3 \\
0.5 \\
12.6 \\
0.0\end{array}$ & $\begin{array}{r}3.3 \\
1.1 \\
1.9 \\
13.7 \\
0.8 \\
46.4 \\
11.5 \\
1.4 \\
19.9 \\
0.0\end{array}$ & $\begin{array}{r}0.0 \\
0.0 \\
0.0 \\
0.0 \\
0.0 \\
0.0 \\
0.0 \\
0.0 \\
0.0 \\
99.7\end{array}$ & $\begin{array}{l}0.0 \\
0.0 \\
0.0\end{array}$ \\
\hline
\end{tabular}

30 Degreas North Letitude

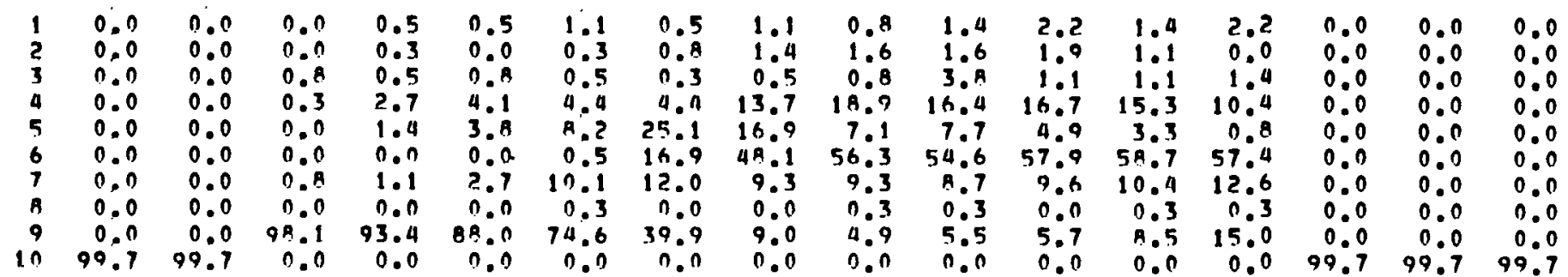


1972

27 Degreas North Lotitude

\begin{tabular}{|c|c|c|c|c|c|c|c|c|c|c|c|c|c|c|c|c|c|}
\hline P T & 126 & 122 & 118 & 114 & 110 & 106 & $\begin{array}{l}\text { Deor } \\
102\end{array}$ & 96 & & $\begin{array}{l}\operatorname{Lons} \\
94\end{array}$ & $\begin{array}{l}\text { eutde } \\
90\end{array}$ & 86 & 82 & 78 & 74 & 70 & 6 \\
\hline $\begin{array}{l}1 \\
? \\
3 \\
4 \\
5 \\
6 \\
7 \\
5\end{array}$ & $\begin{array}{l}0.0 \\
0.0 \\
0.0 \\
0.0 \\
0.0 \\
0.0 \\
0.0 \\
0.0 \\
0.0\end{array}$ & $\begin{array}{l}0.0 \\
0.0 \\
0.0 \\
0.0 \\
0.0 \\
0.0 \\
0.0 \\
0.0 \\
0.0 \\
0.0\end{array}$ & $\begin{array}{r}0.0 \\
0.0 \\
0.0 \\
0.0 \\
0.0 \\
0.0 \\
0.0 \\
0.0 \\
0.0 \\
99.7\end{array}$ & $\begin{array}{r}0.0 \\
0.0 \\
0.0 \\
0.0 \\
0.0 \\
0.0 \\
0.0 \\
0.0 \\
0.0 \\
99.7\end{array}$ & $\begin{array}{c}0.0 \\
0.0 \\
0.0 \\
0.0 \\
0.0 \\
0.0 \\
0.0 \\
0.0 \\
0.0 \\
99.7\end{array}$ & $\begin{array}{r}0.0 \\
0.0 \\
0.0 \\
0.0 \\
0.0 \\
0.0 \\
0.0 \\
0.0 \\
0.0 \\
99.7\end{array}$ & $\begin{array}{r}0.3 \\
0.3 \\
0.0 \\
0.8 \\
12.6 \\
13.1 \\
9.3 \\
0.0 \\
63.7 \\
0.0\end{array}$ & $\begin{array}{r}0.3 \\
0.3 \\
0.8 \\
10.4 \\
8.7 \\
54.9 \\
11.7 \\
0.0 \\
12.8 \\
0.0\end{array}$ & $\begin{array}{r}1 \\
0 \\
2 \\
10 \\
2 \\
64 \\
11 \\
0\end{array}$ & : & $\begin{array}{r}0.5 \\
0.5 \\
9.3 \\
1.9 \\
69.7 \\
8.7 \\
0.0 \\
6.6 \\
0.0\end{array}$ & $\begin{array}{r}1.1 \\
0.5 \\
0.3 \\
9.3 \\
1.4 \\
71.3 \\
9.0 \\
0.0 \\
7.1 \\
0.0\end{array}$ & $\begin{array}{r}0.5 \\
0.5 \\
1.4 \\
7.4 \\
0.5 \\
70.5 \\
10.4 \\
0.0 \\
1.7 \\
0.0\end{array}$ & $\begin{array}{r}0 \\
0 \\
0 \\
5 \\
0 \\
70 \\
0 \\
0 \\
13 \\
0\end{array}$ & $\begin{array}{l}0.0 \\
0.0 \\
0.0 \\
0.0 \\
0.0 \\
0.0 \\
9.97\end{array}$ & $\begin{array}{c}0 \\
0 \\
0 \\
0 \\
0 \\
0 \\
0 \\
0 \\
0 \\
99\end{array}$ & $\begin{array}{l}.0 \\
0 \\
.0 \\
.0 \\
0 \\
0 \\
0 \\
0 \\
0\end{array}$ \\
\hline
\end{tabular}

24 Dograes North Lotitude

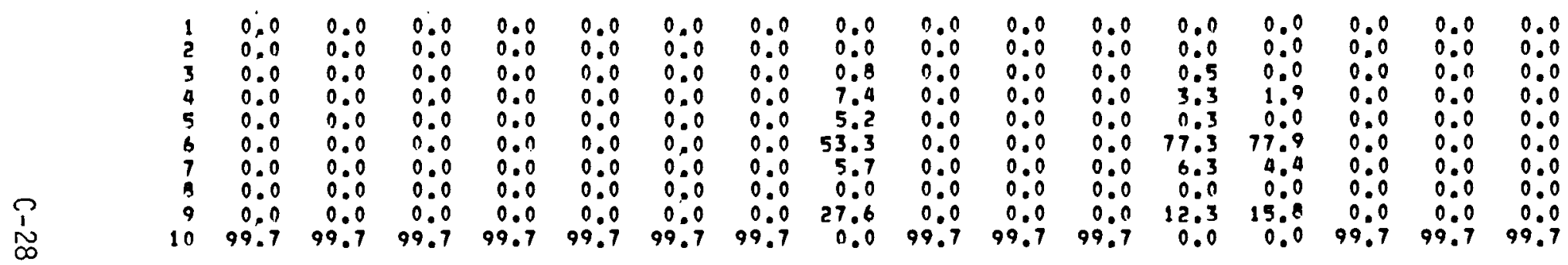


1973

51 Deoroes North Let Itude

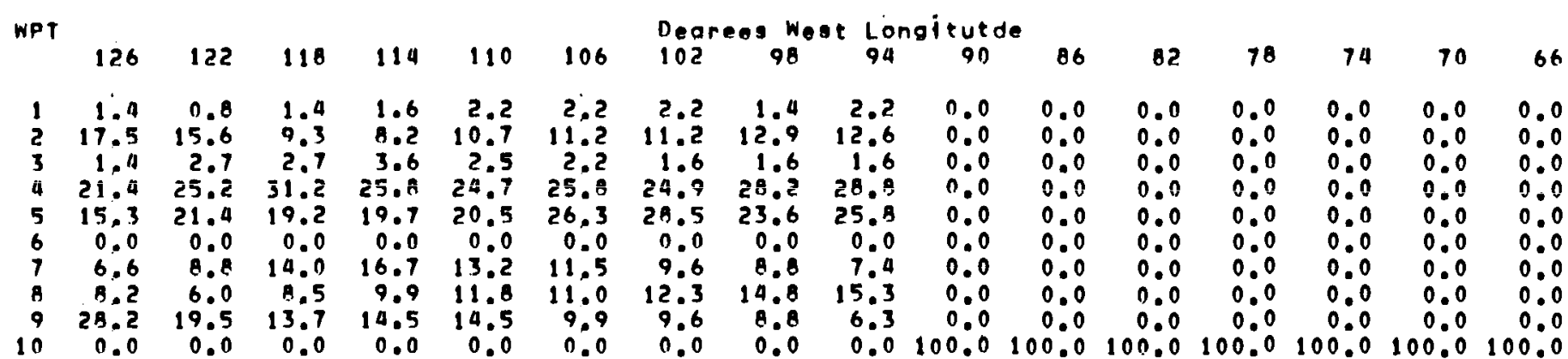

48 Dearees North Lot Itude

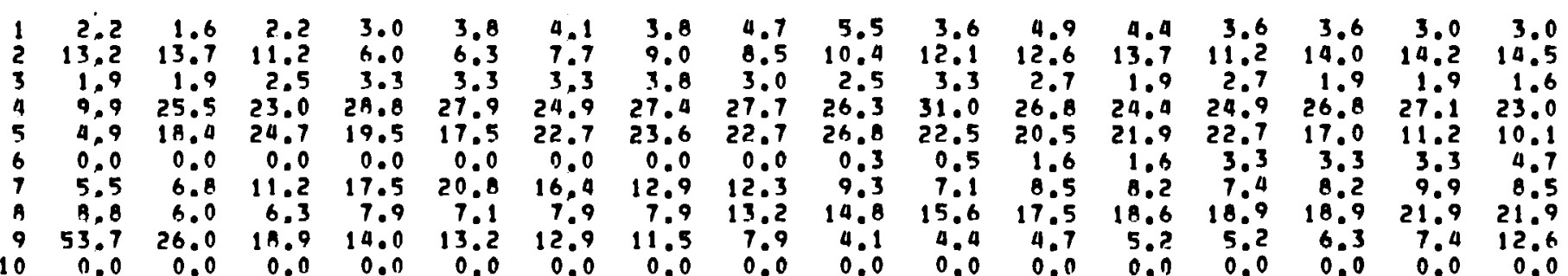

45 Degrees North Lett tude

\begin{tabular}{|c|c|c|c|c|c|c|c|c|c|c|c|c|c|c|c|c|}
\hline $\begin{array}{l}1 \\
2 \\
3 \\
4 \\
5 \\
6 \\
7 \\
8 \\
9 \\
10\end{array}$ & $\begin{array}{r}2.2 \\
7.7 \\
3.0 \\
3.3 \\
3.3 \\
0.0 \\
4.7 \\
10.4 \\
65.5 \\
0.0\end{array}$ & $\begin{array}{r}1.9 \\
7.9 \\
1.6 \\
26.0 \\
16.4 \\
0.0 \\
7.1 \\
5.5 \\
33.4 \\
0.0\end{array}$ & $\begin{array}{r}1.6 \\
7.1 \\
1.9 \\
24.4 \\
26.3 \\
0.0 \\
6.3 \\
4.4 \\
27.9 \\
0.0\end{array}$ & $\begin{array}{r}1.1 \\
5.5 \\
3.3 \\
25.5 \\
31.8 \\
0.0 \\
9.0 \\
4.9 \\
18.9 \\
0.0\end{array}$ & $\begin{array}{r}2.7 \\
5.2 \\
2.7 \\
33.4 \\
20.0 \\
0.0 \\
15.9 \\
3.3 \\
16.7 \\
0.0\end{array}$ & $\begin{array}{r}4.4 \\
6.3 \\
2.5 \\
31.5 \\
18.1 \\
0.0 \\
19.2 \\
3.8 \\
14.2 \\
0.0\end{array}$ & $\begin{array}{r}4.1 \\
6.0 \\
2.7 \\
26.0 \\
21.4 \\
0.0 \\
20.3 \\
6.6 \\
12.9 \\
0.0\end{array}$ & $\begin{array}{r}5.5 \\
6.3 \\
3.3 \\
27.1 \\
24.7 \\
0.0 \\
15.1 \\
10.4 \\
7.7 \\
0.0\end{array}$ & $\begin{array}{r}4.9 \\
9.9 \\
4.1 \\
26.3 \\
28.8 \\
1.4 \\
7.9 \\
12.3 \\
4.4 \\
0.0\end{array}$ & $\begin{array}{r}4.1 \\
11.8 \\
4.1 \\
27.9 \\
25.5 \\
2.5 \\
7.1 \\
13.4 \\
3.6 \\
0.0\end{array}$ & $\begin{array}{r}5.8 \\
11.8 \\
1.9 \\
29.3 \\
22.7 \\
4.1 \\
1.2 \\
12.1 \\
4.1 \\
0.0\end{array}$ & $\begin{array}{r}6.0 \\
11.0 \\
1.1 \\
23.8 \\
24.7 \\
5.5 \\
8.2 \\
15.9 \\
3.9 \\
0.9\end{array}$ & $\begin{array}{r}3.6 \\
14.5 \\
4.7 \\
20.4 \\
20.8 \\
4.7 \\
0.5 \\
14.2 \\
9.7 \\
0.0\end{array}$ & $\begin{array}{r}4.4 \\
12.3 \\
3.3 \\
26.3 \\
15.9 \\
5.5 \\
8.8 \\
16.7 \\
6.8 \\
0.0\end{array}$ & $\begin{array}{r}4.7 \\
12.6 \\
2.2 \\
26.6 \\
10.4 \\
6.3 \\
8.2 \\
18.9 \\
10.1 \\
0.0\end{array}$ & $\begin{array}{r}3.6 \\
11.0 \\
2.5 \\
20.0 \\
4.9 \\
9.6 \\
9.9 \\
21.6 \\
16.2 \\
0.0\end{array}$ \\
\hline
\end{tabular}

42 Dearees North Latt tude

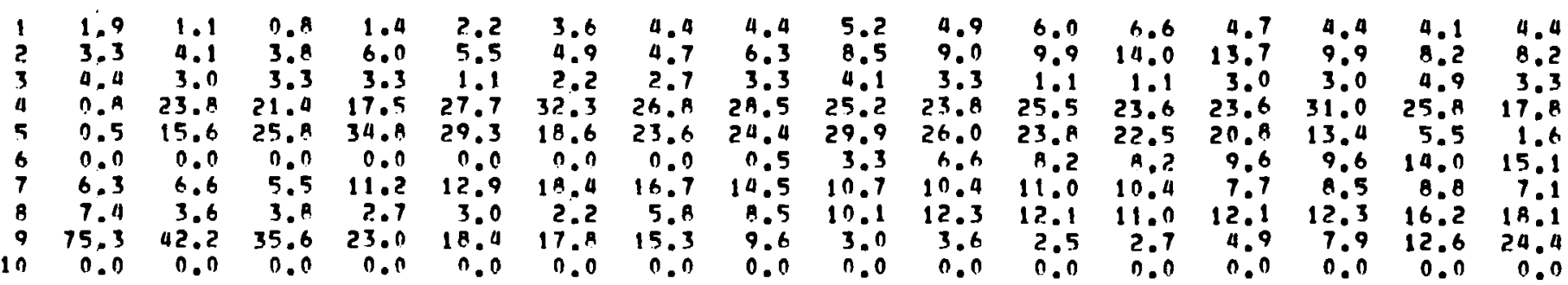


1973

39 Degrees North Lot ltude

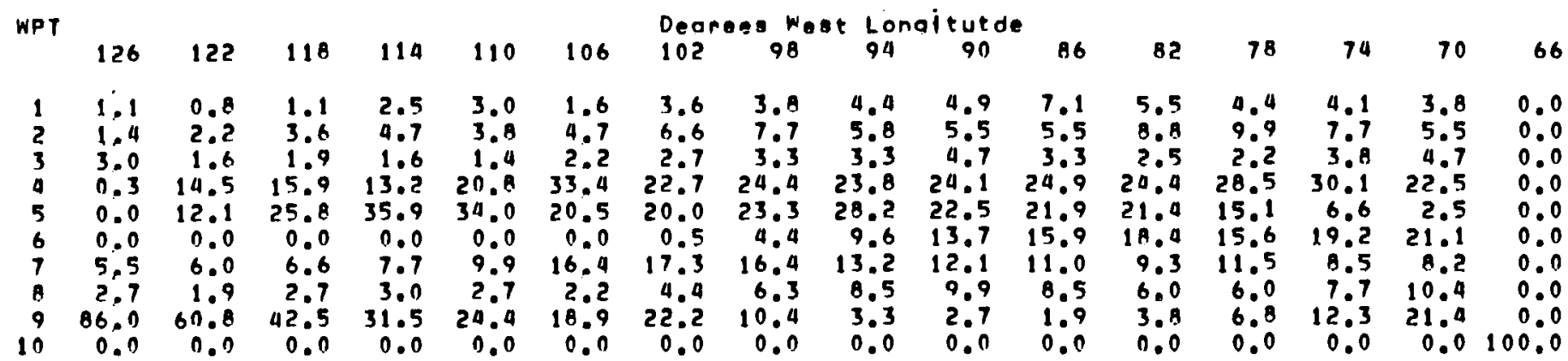

36 Dogrese North Let Itude

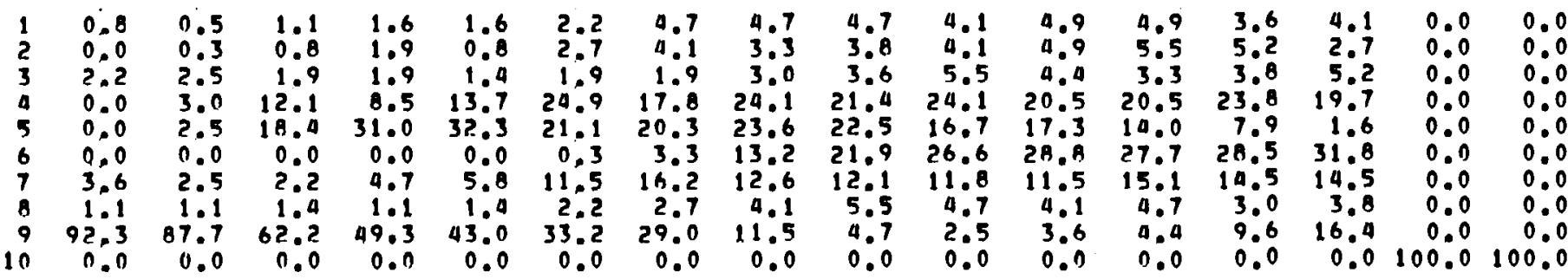

33 Degrees North Lot ltude

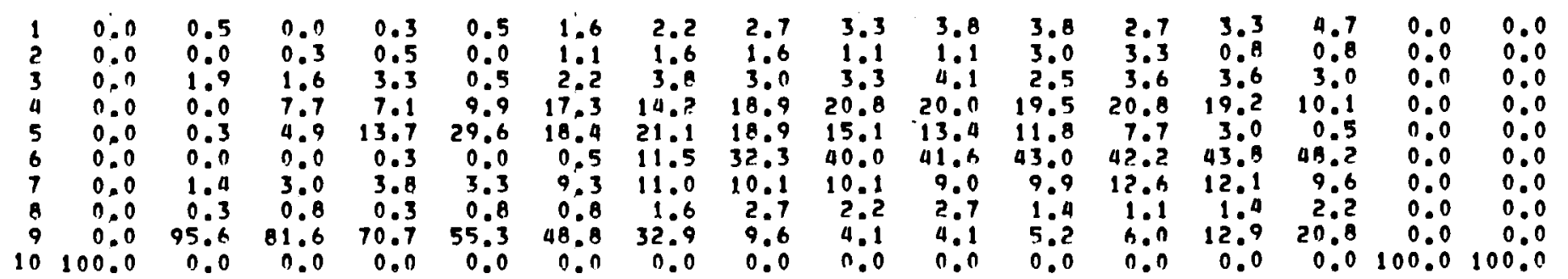

30 Degrees North Lattude

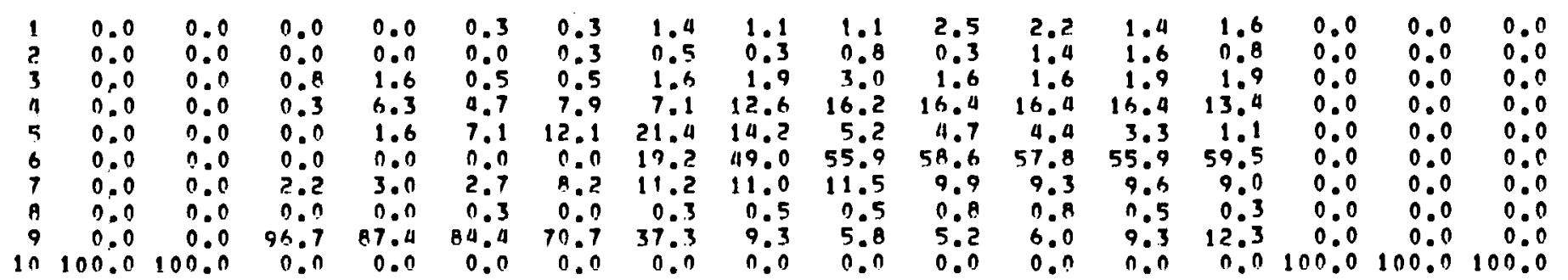


1973

27 Degrass North Lattude

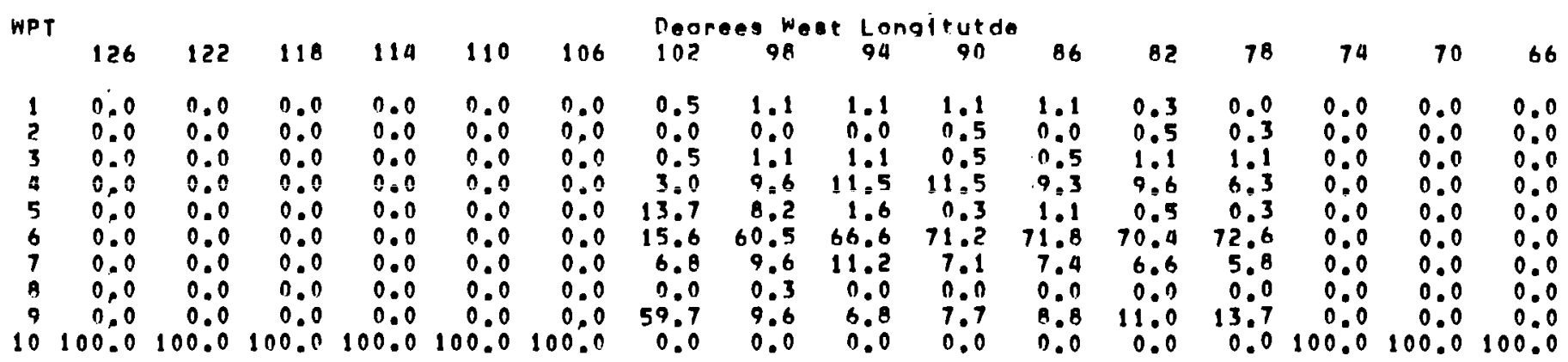

24 Degreas North Lat ltude

$\begin{array}{rrrrrrrrrrrrrrrrrr}1 & 0.0 & 0.0 & 0.0 & 0.0 & 0.0 & 0.0 & 0.0 & 0.3 & 0.0 & 0.0 & 0.0 & 0.5 & 0.5 & 0.0 & 0.0 & 0.0 \\ 2 & 0.0 & 0.0 & 0.0 & 0.0 & 0.0 & 0.0 & 0.0 & 0.0 & 0.0 & 0.0 & 0.0 & 0.0 & 0.0 & 0.0 & 0.0 & 0.0 \\ 3 & 0.0 & 0.0 & 0.0 & 0.0 & 0.0 & 0.0 & 0.0 & 0.3 & 0.0 & 0.0 & 0.0 & 0.3 & 0.5 & 0.0 & 0.0 & 0.0 \\ 4 & 0.0 & 0.0 & 0.0 & 0.0 & 0.0 & 0.0 & 0.0 & 5.0 & 0.0 & 0.0 & 0.0 & 3.8 & 2.5 & 0.0 & 0.0 & 0.0 \\ 5 & 0.0 & 0.0 & 0.0 & 0.0 & 0.0 & 0.0 & 0.0 & 4.9 & 0.0 & 0.0 & 0.0 & 0.0 & 0.0 & 0.0 & 0.0 & 0.0 \\ 6 & 0.0 & 0.0 & 0.0 & 0.0 & 0.0 & 0.0 & 0.0 & 59.7 & 0.0 & 0.0 & 0.0 & 77.0 & 77.3 & 0.0 & 0.0 & 0.0 \\ 7 & 0.0 & 0.0 & 0.0 & 0.0 & 0.0 & 0.0 & 0.0 & 7.4 & 0.0 & 0.0 & 0.0 & 5.0 & 4.7 & 0.0 & 0.0 & 0.0 \\ 8 & 0.0 & 0.0 & 0.0 & 0.0 & 0.0 & 0.0 & 0.0 & 0.0 & 0.0 & 0.0 & 0.0 & 0.0 & 0.0 & 0.0 & 0.0 & 0.0 \\ 9 & 0.0 & 0.0 & 0.0 & 0.0 & 0.0 & 0.0 & 0.0 & 21.0 & 0.0 & 0.0 & 0.0 & 12.0 & 14.5 & 0.0 & 0.0 & 0.0 \\ 10 & 100.0 & 100.0 & 100.0 & 100.0 & 100.0 & 100.0 & 100.0 & 0.0 & 100.0 & 100.0 & 100.0 & 0.0 & 0.0 & 100.0 & 100.0 & 100.0\end{array}$

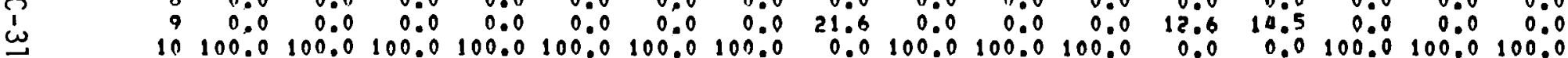


51 Dearese North Letltude

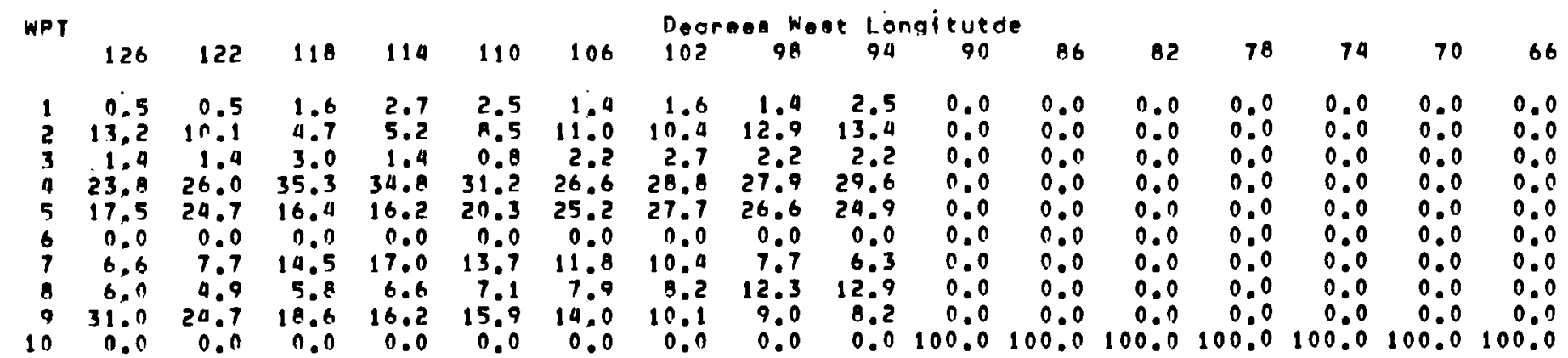

48 Degrees North Letitude

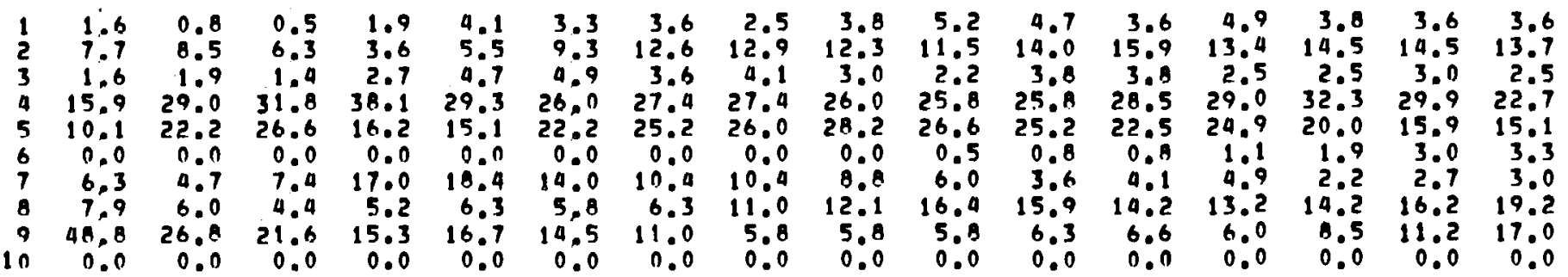

45 Dearees North Letltude

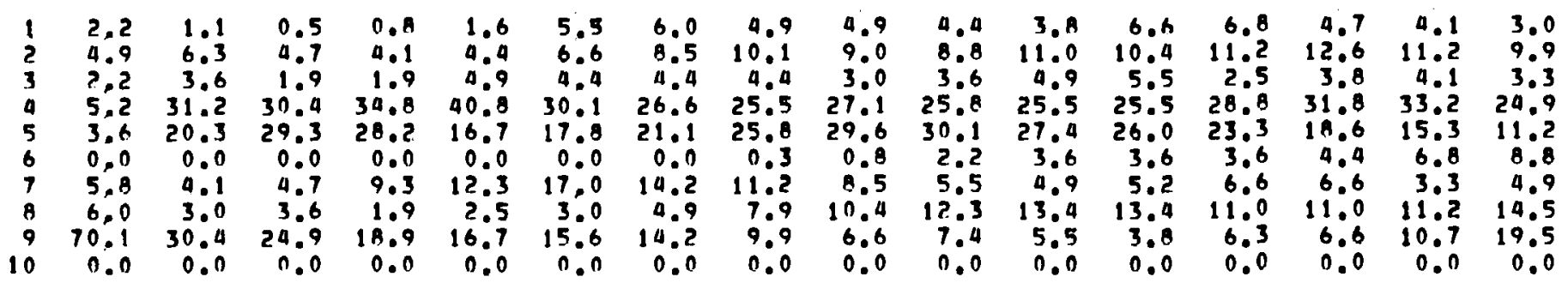

42 Depraee North Latteude

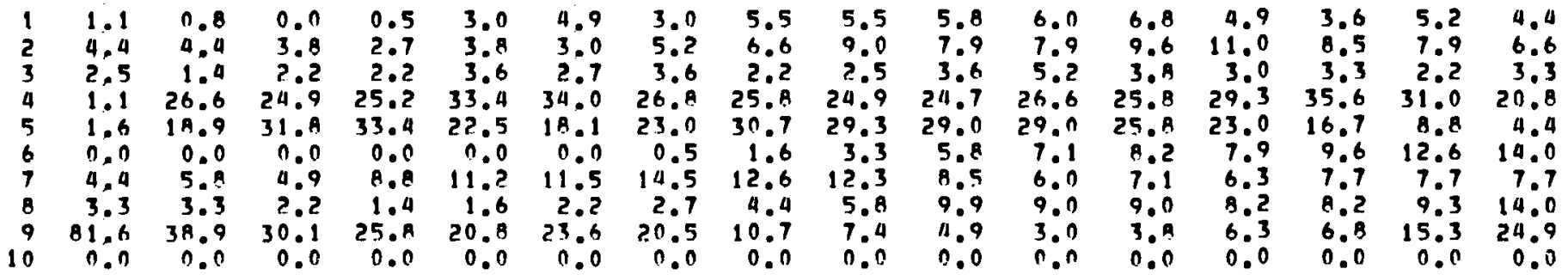


1974

39 Dearees North Lotteudo

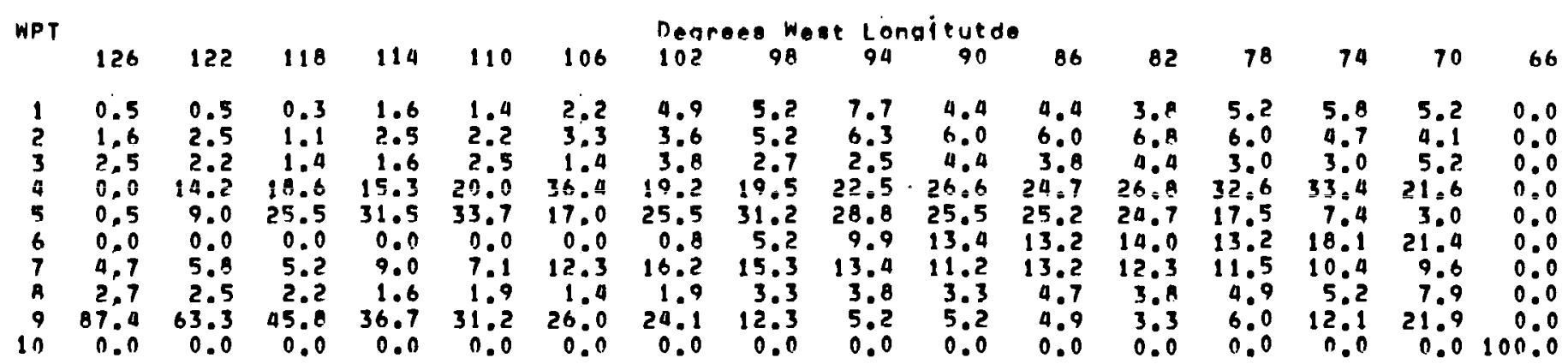

36 Degrees North Letftude

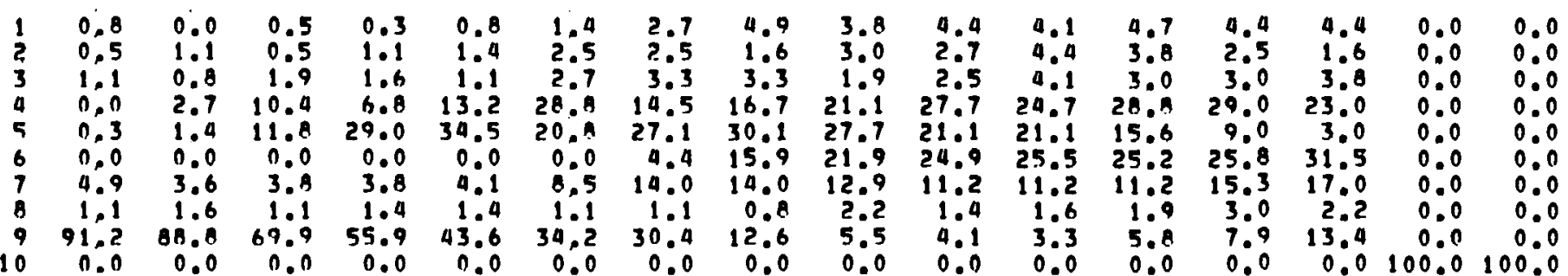

33 Degrees North Lotitude

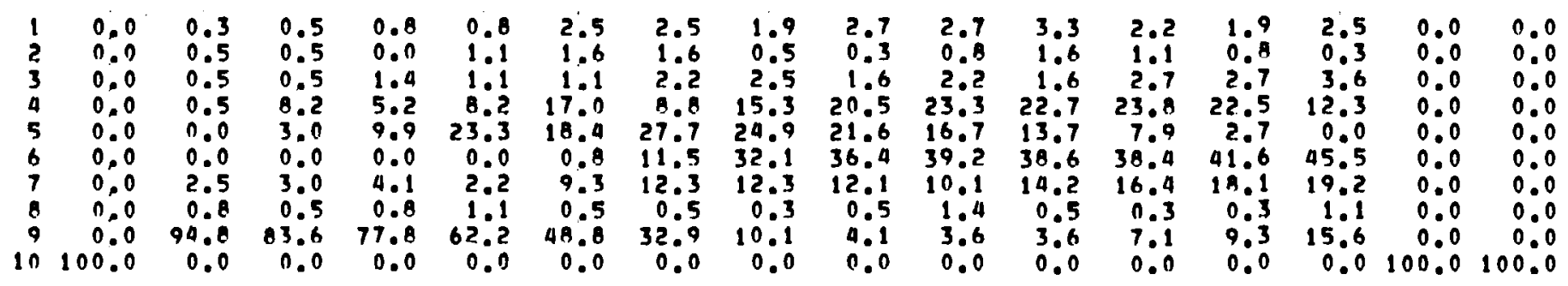

30 nearees North Letltude

\begin{tabular}{|c|c|c|c|c|c|c|c|c|c|c|c|c|c|c|c|c|}
\hline $\begin{array}{l}1 \\
2 \\
3 \\
4 \\
5 \\
6 \\
7 \\
8 \\
9 \\
0\end{array}$ & $\begin{array}{r}0.0 \\
0.0 \\
0.0 \\
0.0 \\
0.0 \\
0.0 \\
0.0 \\
0.0 \\
0.0 \\
0.0 \\
0.0\end{array}$ & $\begin{array}{r}0.0 \\
0.0 \\
0.0 \\
0.0 \\
0.0 \\
0.0 \\
0.0 \\
0.0 \\
0.0 \\
100.0\end{array}$ & $\begin{array}{r}0.3 \\
0.0 \\
0.8 \\
0.8 \\
0.3 \\
0.0 \\
0.0 \\
0.0 \\
97.0 \\
0.0\end{array}$ & $\begin{array}{r}0.3 \\
0.0 \\
1.4 \\
3.0 \\
1.0 \\
0.0 \\
1.0 \\
0.0 \\
92.6 \\
0.0\end{array}$ & $\begin{array}{r}1.1 \\
0.5 \\
0.3 \\
1.9 \\
5.2 \\
0.3 \\
3.3 \\
0.0 \\
87.0 \\
0.0\end{array}$ & $\begin{array}{r}1.6 \\
0.5 \\
0.0 \\
11.7 \\
7.9 \\
1.9 \\
5.8 \\
0.3 \\
77.8 \\
0.0\end{array}$ & $\begin{array}{r}1.1 \\
1.1 \\
0.3 \\
3.8 \\
23.3 \\
18.4 \\
10.7 \\
0.0 \\
41.11 \\
0.0\end{array}$ & $\begin{array}{r}0.5 \\
0.0 \\
0.5 \\
14.0 \\
19.4 \\
47.4 \\
10.7 \\
0.0 \\
0.5 \\
0.0\end{array}$ & $\begin{array}{r}0.8 \\
0.0 \\
2.2 \\
17.8 \\
8.2 \\
52.6 \\
14.0 \\
0.0 \\
4.4 \\
0.0\end{array}$ & $\begin{array}{r}1.4 \\
0.3 \\
1.9 \\
15.9 \\
7.9 \\
53.4 \\
15.1 \\
0.0 \\
4.1 \\
0.0\end{array}$ & $\begin{array}{r}1.4 \\
0.8 \\
0.8 \\
18.1 \\
7.1 \\
53.7 \\
13.4 \\
0.0 \\
4.7 \\
0.0\end{array}$ & $\begin{array}{r}1.1 \\
0.8 \\
1.1 \\
1.04 \\
2.5 \\
57.5 \\
12.3 \\
0.0 \\
6.3 \\
0.0\end{array}$ & $\begin{array}{r}1.1 \\
0.3 \\
1.4 \\
13.4 \\
0.8 \\
60.0 \\
12.6 \\
0.3 \\
10.1 \\
0.0\end{array}$ & $\begin{array}{r}0.0 \\
0.0 \\
0.0 \\
0.0 \\
0.0 \\
0.0 \\
0.0 \\
0.0 \\
0.0 \\
100.0\end{array}$ & $\begin{array}{rl}0.0 & 0 \\
0.0 \\
0.0 \\
0.0 \\
0.0 \\
0.0 \\
0.0 \\
0.00 \\
0.0 \\
100.0\end{array}$ & $\begin{array}{r}0.0 \\
0.0 \\
0.0 \\
0.0 \\
0.0 \\
0.0 \\
0.0 \\
0.0 \\
0.0 \\
100.0\end{array}$ \\
\hline
\end{tabular}


1974

27 Dogreas North Lat 1 tude

\begin{tabular}{|c|c|c|c|c|c|c|c|c|c|c|c|c|c|c|c|c|}
\hline WPT & 126 & 122 & 118 & 114 & 110 & 106 & $\begin{array}{l}0_{00 r} \\
102\end{array}$ & $=3 W_{98}^{W_{6}}$ & $\begin{array}{l}\text { Lor } \\
94\end{array}$ & $\begin{array}{c}\text { itutde } \\
90\end{array}$ & 86 & 82 & 78 & 74 & 70 & 66 \\
\hline $\begin{array}{r}1 \\
2 \\
3 \\
4 \\
5 \\
6 \\
7 \\
6 \\
9 \\
10\end{array}$ & $\begin{aligned} 0.0 \\
0.0 \\
0.0 \\
0.0 \\
0.0 \\
0.0 \\
0.0 \\
0.0 \\
0.0 \\
100.0\end{aligned}$ & $\begin{array}{r}0.0 \\
0.0 \\
0.0 \\
0.0 \\
0.0 \\
0.0 \\
0.0 \\
0.0 \\
0.0 \\
100.0\end{array}$ & $\begin{array}{r}0.0 \\
0.0 \\
0.0 \\
0.0 \\
0.0 \\
0.0 \\
0.0 \\
0.0 \\
0.0 \\
100.0\end{array}$ & $\begin{array}{r}0.0 \\
0.0 \\
0.0 \\
0.0 \\
0.0 \\
0.0 \\
0.0 \\
0.0 \\
0.0 \\
100.0\end{array}$ & $\begin{array}{r}0.0 \\
0.0 \\
0.0 \\
0.0 \\
0.0 \\
0.0 \\
0.0 \\
0.0 \\
0.0 \\
100.0\end{array}$ & $\begin{array}{r}0.0 \\
0.0 \\
0.0 \\
0.0 \\
0.0 \\
0.0 \\
0.0 \\
0.0 \\
0.0 \\
100.0\end{array}$ & $\begin{array}{r}0.0 \\
0.3 \\
0.3 \\
1.9 \\
13.2 \\
15.1 \\
9.3 \\
0.0 \\
60.0 \\
0.0\end{array}$ & $\begin{array}{r}0.3 \\
0.0 \\
0.3 \\
10.4 \\
7.7 \\
54.5 \\
14.0 \\
0.0 \\
12.9 \\
0.0\end{array}$ & $\begin{array}{l}0.3 \\
0.0 \\
1.6 \\
9.3 \\
2.5 \\
63.6 \\
16.2 \\
0.0 \\
6.6 \\
0.0\end{array}$ & $\begin{array}{l}1.6 \\
0.0 \\
0.0 \\
9.0 \\
1.6 \\
68.2 \\
12.6 \\
0.0 \\
6.8 \\
0.0\end{array}$ & $\begin{array}{r}0.8 \\
0.0 \\
0.3 \\
9.3 \\
1.6 \\
70.4 \\
10.1 \\
0.0 \\
7.4 \\
0.0\end{array}$ & $\begin{array}{l}0.5 \\
0.0 \\
0.8 \\
0.3 \\
0.5 \\
70.7 \\
10.4 \\
0.3 \\
7.4 \\
0.0\end{array}$ & $\begin{array}{r}0.5 \\
0.0 \\
1.1 \\
5.2 \\
0.0 \\
74.5 \\
7.9 \\
0.0 \\
10.7 \\
0.0\end{array}$ & $\begin{array}{r}0.0 \\
0.0 \\
0.0 \\
0.0 \\
0.0 \\
0.0 \\
0.0 \\
0.0 \\
0.0 \\
100.0\end{array}$ & $\begin{array}{r}0.0 \\
0.0 \\
0.0 \\
0.0 \\
0.0 \\
0.0 \\
0.0 \\
0.0 \\
0.0 \\
100.0\end{array}$ & $\begin{aligned} 0.0 \\
0.0 \\
0.0 \\
0.0 \\
0.0 \\
0.0 \\
0.0 \\
0.0 \\
0.0 \\
100.0\end{aligned}$ \\
\hline
\end{tabular}

24 Doarseg North Letitude

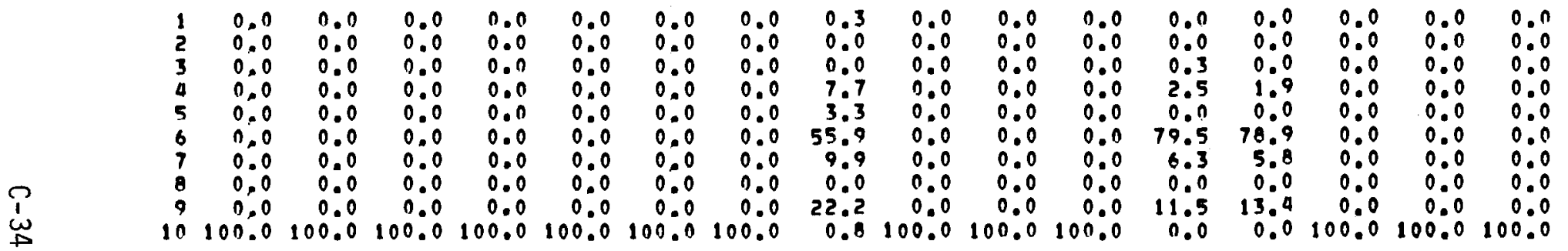


1975

51 Degreas North Letitude

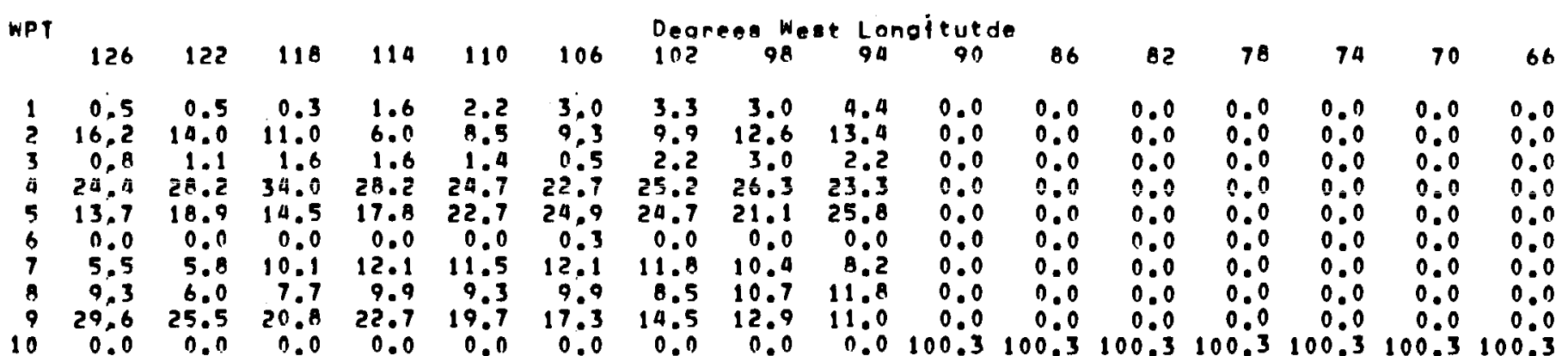

48 Dogrees North Letftude

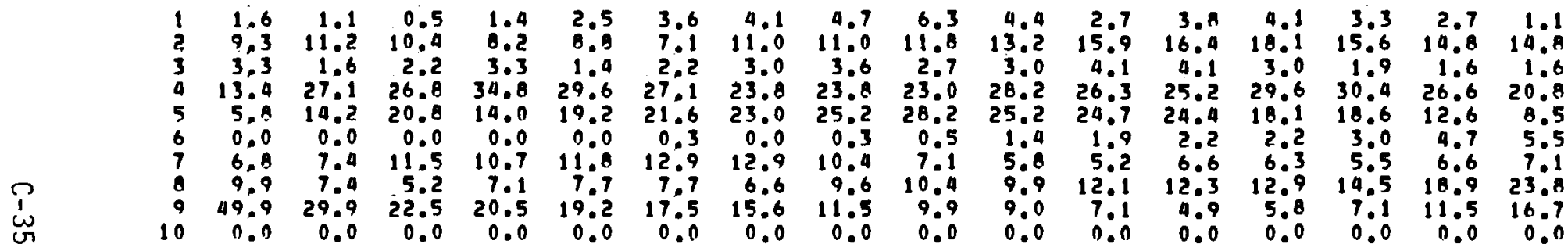

45 Dearees North Letftude

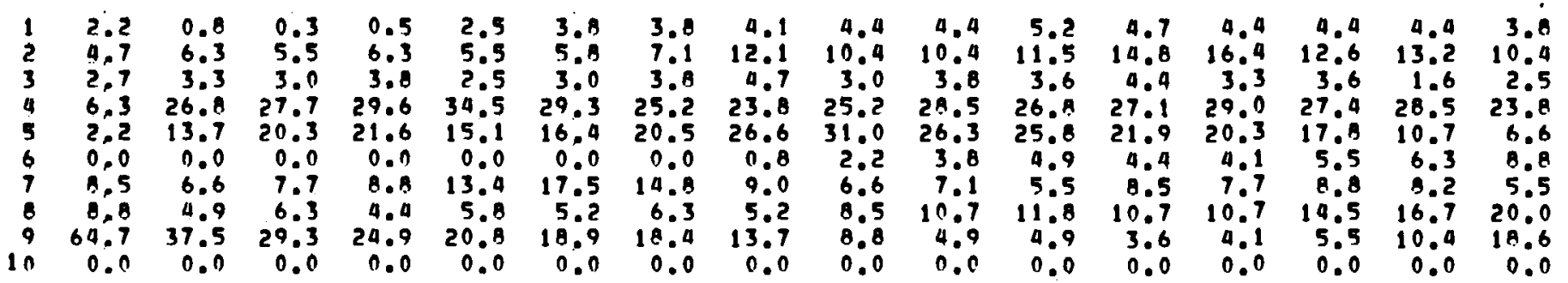

42 Degrees North Letitude

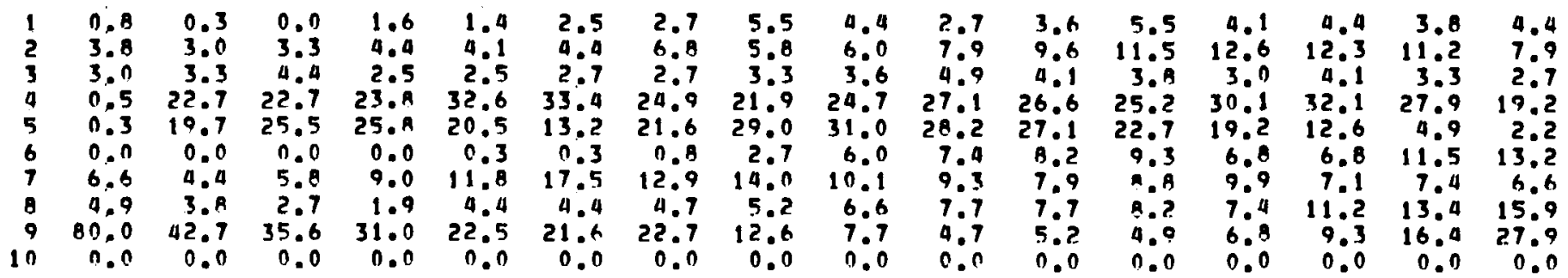


1975

39 Deqrees North Lotltude

\begin{tabular}{|c|c|c|c|c|c|c|c|c|c|c|c|c|c|c|c|c|}
\hline WPT & 126 & 122 & 118 & 114 & 110 & 106 & $\begin{array}{l}\text { Degr } \\
102\end{array}$ & 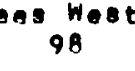 & $\begin{array}{l}t \text { Lor } \\
94\end{array}$ & $\begin{array}{c}\text { gitut do } \\
90\end{array}$ & 86 & 82 & 78 & 74 & 70 & 66 \\
\hline $\begin{array}{r}1 \\
2 \\
3 \\
4 \\
5 \\
6 \\
7 \\
6 \\
9 \\
10\end{array}$ & $\begin{array}{r}0.5 \\
1.4 \\
1.9 \\
0.3 \\
0.5 \\
0.0 \\
5.2 \\
3.3 \\
66.8 \\
0.0\end{array}$ & $\begin{array}{r}0.5 \\
1.6 \\
3.6 \\
11.0 \\
12.1 \\
0.0 \\
5.2 \\
1.9 \\
64.1 \\
0.0\end{array}$ & $\begin{array}{r}1.4 \\
2.2 \\
2.7 \\
15.9 \\
25.5 \\
0.0 \\
4.7 \\
2.2 \\
45.5 \\
0.0\end{array}$ & $\begin{array}{r}2.5 \\
3.0 \\
1.9 \\
16.7 \\
32.3 \\
0.0 \\
5.8 \\
2.2 \\
35.6 \\
0.0\end{array}$ & $\begin{array}{r}1.4 \\
3.8 \\
2.5 \\
20.8 \\
31.5 \\
0.3 \\
5.8 \\
3.3 \\
30.7 \\
0.0\end{array}$ & $\begin{array}{r}3.3 \\
4.1 \\
1.9 \\
39.2 \\
11.8 \\
1.1 \\
12.6 \\
3.3 \\
22.7 \\
0.0\end{array}$ & $\begin{array}{r}3.6 \\
4.4 \\
2.5 \\
19.2 \\
22.5 \\
1.9 \\
18.4 \\
3.0 \\
24.7 \\
0.0\end{array}$ & $\begin{array}{r}3.8 \\
5.2 \\
1.4 \\
22.7 \\
26.3 \\
7.7 \\
15.3 \\
3.0 \\
14.5 \\
0.0\end{array}$ & $\begin{array}{r}5.5 \\
4.4 \\
3.3 \\
22.5 \\
27.4 \\
11.8 \\
12.9 \\
5.2 \\
7.1 \\
0.0\end{array}$ & $\begin{array}{r}3.3 \\
5.5 \\
4.7 \\
23.6 \\
29.0 \\
14.8 \\
11.0 \\
4.7 \\
3.6 \\
0.0\end{array}$ & $\begin{array}{r}4.4 \\
7.1 \\
3.6 \\
24.0 \\
25.5 \\
15.3 \\
12.1 \\
4.7 \\
3.0 \\
0.0\end{array}$ & $\begin{array}{r}5.8 \\
7.0 \\
3.6 \\
24.1 \\
22.7 \\
15.1 \\
10.4 \\
4.7 \\
5.8 \\
0.0\end{array}$ & $\begin{array}{r}5.8 \\
6.8 \\
3.6 \\
29.9 \\
15.3 \\
12.1 \\
11.5 \\
5.8 \\
9.3 \\
0.0\end{array}$ & $\begin{array}{r}5.5 \\
7.4 \\
5.5 \\
31.2 \\
5.8 \\
14.2 \\
10.7 \\
8.5 \\
11.2 \\
0.0\end{array}$ & $\begin{array}{r}6.0 \\
4.9 \\
4.7 \\
20.8 \\
1.4 \\
19.6 \\
9.0 \\
9.9 \\
24.7 \\
0.0\end{array}$ & $\begin{array}{r}0.0 \\
0.0 \\
0.0 \\
0.0 \\
0.0 \\
0.0 \\
0.0 \\
0.0 \\
0.0 \\
100.3\end{array}$ \\
\hline
\end{tabular}

36 Deorese North Lotitude

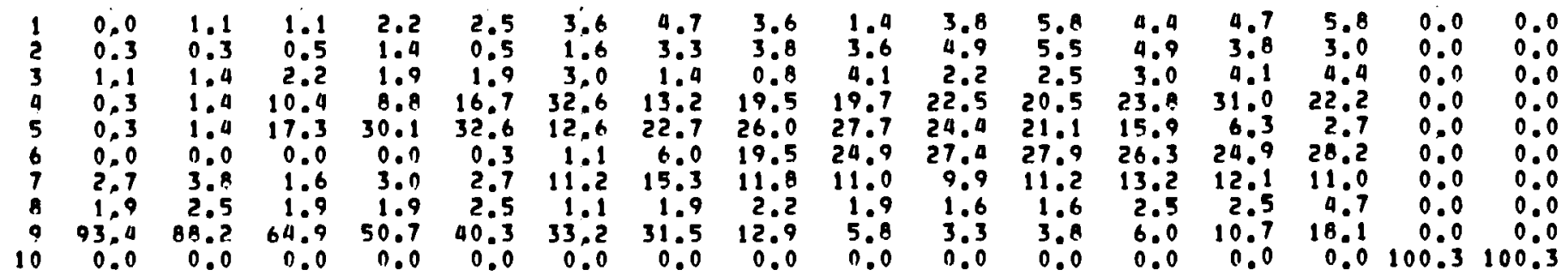

33 Degrees North Latltude

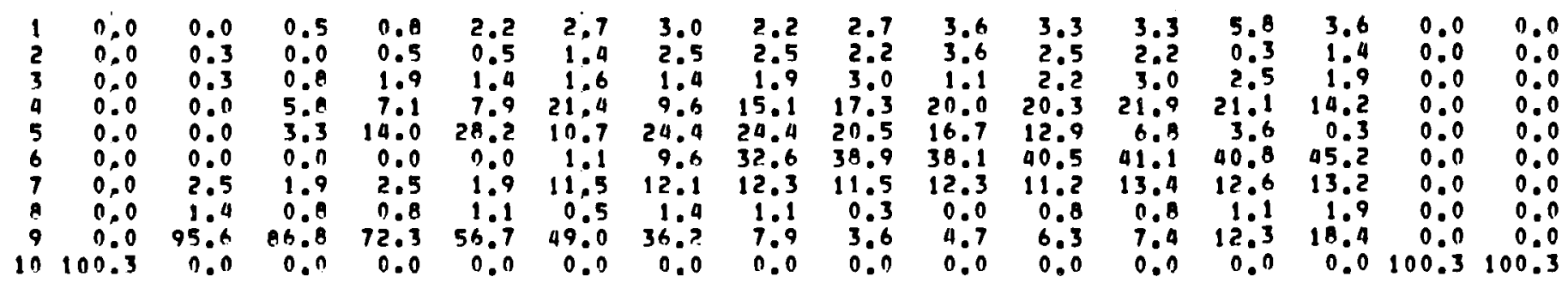

30 Degreas North Lat Itude

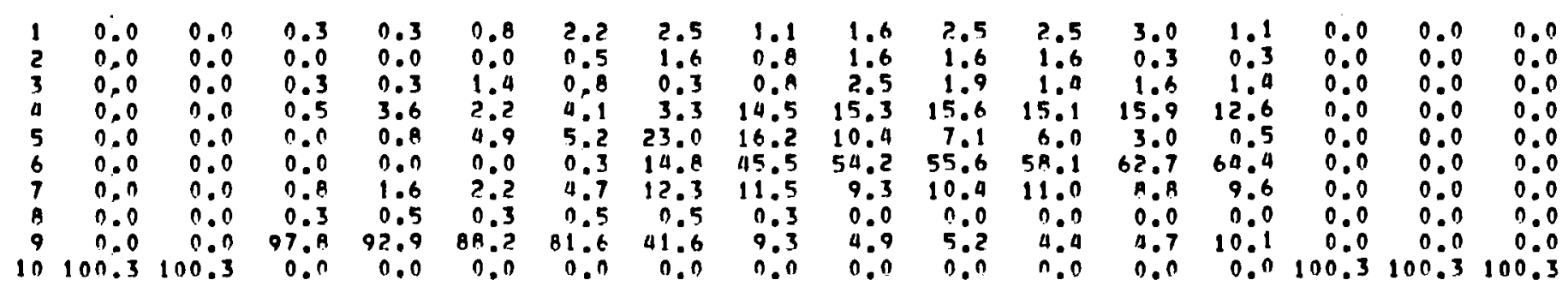


1975

27 Dogrees North Letitude

\begin{tabular}{|c|c|c|c|c|c|c|c|c|c|c|c|c|c|c|c|c|c|}
\hline WPT & 126 & 122 & 118 & 114 & 110 & 106 & $\begin{array}{l}\text { Deg } \\
10 ?\end{array}$ & $9 B$ & & $\begin{array}{l}\text { Lone } \\
\text { 94 }\end{array}$ & $\begin{array}{c}\text { gitut de } \\
90\end{array}$ & 86 & 82 & 78 & 74 & 70 & 36 \\
\hline $\begin{array}{l}1 \\
2 \\
3 \\
4 \\
5 \\
6 \\
7 \\
0 \\
9 \\
10\end{array}$ & $\begin{array}{ll}0.0 & 0 \\
0.0 & 0 \\
0.0 & 0 \\
0.0 & 0 \\
0.0 & 0 \\
0.0 & 0 \\
0.0 & 0 \\
0.0 & 0 \\
0.00 \\
0 & 0.3\end{array}$ & $\begin{array}{l}0.0 \\
0.0 \\
0.0 \\
0.0 \\
0.0 \\
0.0 \\
0.0 \\
0.0 \\
0.0 \\
00.3\end{array}$ & $\begin{array}{r}0.0 \\
0.0 \\
0.0 \\
0.0 \\
0.0 \\
0.0 \\
0.0 \\
0.0 \\
0.0 \\
100.3\end{array}$ & $\begin{array}{r}0.0 \\
0.0 \\
0.0 \\
0.0 \\
0.0 \\
0.0 \\
0.0 \\
0.0 \\
0.0 \\
100.3\end{array}$ & $\begin{array}{l}0.0 \\
0.0 \\
0.0 \\
0.0 \\
0.0 \\
0.0 \\
0.0 \\
0.0 \\
0.0 \\
00.3\end{array}$ & $\begin{array}{r}0.0 \\
0.0 \\
0.0 \\
0.0 \\
0.0 \\
0.0 \\
0.0 \\
0.0 \\
0.0 \\
100.3\end{array}$ & $\begin{array}{r}1.4 \\
0.3 \\
0.3 \\
1.1 \\
13.7 \\
9.6 \\
6.0 \\
0.0 \\
67.7 \\
0.0\end{array}$ & $\begin{array}{r}1.1 \\
0.0 \\
0.5 \\
11.8 \\
10.1 \\
53.7 \\
8.5 \\
0.0 \\
14.2 \\
0.0\end{array}$ & $\begin{array}{r}2 \\
0 \\
1 \\
11 \\
3 \\
64 \\
9 \\
0 \\
8 \\
0\end{array}$ & $\begin{array}{l}1 \\
.5 \\
0 \\
1 \\
6 \\
0 \\
0 \\
5 \\
0\end{array}$ & $\begin{array}{r}2.5 \\
0.0 \\
0.5 \\
10.4 \\
1.6 \\
68.6 \\
7.9 \\
0.0 \\
8.2 \\
0.0\end{array}$ & $\begin{array}{r}0.8 \\
0.5 \\
0.5 \\
9.0 \\
0.8 \\
72.3 \\
0.2 \\
0.0 \\
7.7 \\
0.0\end{array}$ & $\begin{array}{r}0.5 \\
0.3 \\
0.3 \\
0.8 \\
0.5 \\
74.0 \\
7.4 \\
0.0 \\
8.2 \\
0.0\end{array}$ & $\begin{array}{r}0.0 \\
0.0 \\
0.8 \\
6.3 \\
0.0 \\
76.2 \\
6.8 \\
0.0 \\
9.9 \\
0.0\end{array}$ & $\begin{array}{l}0.0 \\
0.0 \\
0.0 \\
0.0 \\
0.0 \\
0.0 \\
0.0 \\
0.0 \\
0.0 \\
100.3\end{array}$ & $\begin{array}{l}0.0 \\
0.0 \\
0.0 \\
0.0 \\
0.0 \\
0.0 \\
0.0 \\
0.0 \\
0.0 \\
00.3\end{array}$ & $\begin{array}{l}0 \\
0 \\
0 \\
0 \\
0 \\
0 \\
0\end{array}$ \\
\hline
\end{tabular}

24 Dearees North Latleude

$\begin{array}{rrrrrrrrrrrrrrrrr}1 & 0.0 & 0.0 & 0.0 & 0.0 & 0.0 & 0.0 & 0.0 & 0.3 & 0.0 & 0.0 & 0.0 & 0.0 & 0.0 & 0.0 & 0.0 & 0.0 \\ 2 & 0.0 & 0.0 & 0.0 & 0.0 & 0.0 & 0.0 & 0.0 & 0.0 & 0.0 & 0.0 & 0.0 & 0.0 & 0.0 & 0.0 & 0.0 & 0.0 \\ 3 & 0.0 & 0.0 & 0.0 & 0.0 & 0.0 & 0.0 & 0.0 & 0.3 & 0.0 & 0.0 & 0.0 & 0.3 & 0.3 & 0.0 & 0.0 & 0.0 \\ 4 & 0.0 & 0.0 & 0.0 & 0.0 & 0.0 & 0.0 & 0.0 & 0.0 & 0.0 & 0.0 & 0.0 & 3.0 & 1.6 & 0.0 & 0.0 & 0.0 \\ 5 & 0.0 & 0.0 & 0.0 & 0.0 & 0.0 & 0.0 & 0.0 & 6.0 & 0.0 & 0.0 & 0.0 & 0.0 & 0.0 & 0.0 & 0.0 & 0.0 \\ 6 & 0.0 & 0.0 & 0.0 & 0.0 & 0.0 & 0.0 & 0.0 & 47.9 & 0.0 & 0.0 & 0.0 & 00.3 & 01.0 & 0.0 & 0.0 & 0.0 \\ 7 & 0.0 & 0.0 & 0.0 & 0.0 & 0.0 & 0.0 & 0.0 & 4.1 & 0.0 & 0.0 & 0.0 & 4.0 & 4.4 & 0.0 & 0.0 & 0.0 \\ 8 & 0.0 & 0.0 & 0.0 & 0.0 & 0.0 & 0.0 & 0.0 & 0.0 & 0.0 & 0.0 & 0.0 & 0.0 & 0.0 & 0.0 & 0.0 & 0.0 \\ 9 & 0.0 & 0.0 & 0.0 & 0.0 & 0.0 & 0.0 & 0.0 & 31.2 & 0.0 & 0.0 & 0.0 & 11.5 & 12.1 & 0.0 & 0.0 & 0.0 \\ 10 & 100.3 & 100.3 & 100.3 & 100.3 & 100.3 & 100.3 & 100.3 & 0.5 & 100.3 & 100.3 & 100.3 & 0.0 & 0.0 & 100.3 & 100.3 & 100.3\end{array}$


51 Deorees North Lotttude

\begin{tabular}{|c|c|c|c|c|c|c|c|c|c|c|c|c|c|c|c|c|}
\hline NPT & 126 & 122 & 118 & 114 & 110 & 106 & $\begin{array}{l}\text { Degr } \\
102\end{array}$ & $\begin{array}{c}W \\
98\end{array}$ & $\begin{array}{l}\text { Lo } \\
94\end{array}$ & $\begin{array}{l}\text { tutde } \\
90\end{array}$ & 86 & 62 & 78 & 74 & 70 & 66 \\
\hline $\begin{array}{l}1 \\
2 \\
3 \\
4 \\
5 \\
6 \\
7 \\
6 \\
9 \\
10\end{array}$ & $\begin{array}{r}3.0 \\
16.1 \\
1.4 \\
19.3 \\
18.6 \\
0.0 \\
7.4 \\
6.0 \\
29.2 \\
0.0\end{array}$ & $\begin{array}{r}1.9 \\
10.4 \\
1.4 \\
25.1 \\
30.3 \\
0.0 \\
8.2 \\
4.1 \\
19.6 \\
0.0\end{array}$ & $\begin{array}{r}1.9 \\
7.4 \\
0.3 \\
40.4 \\
19.4 \\
0.0 \\
11.5 \\
6.0 \\
13.1 \\
0.0\end{array}$ & $\begin{array}{r}3.0 \\
5.2 \\
0.5 \\
34.4 \\
14.5 \\
0.0 \\
18.9 \\
6.8 \\
16.7 \\
0.0\end{array}$ & $\begin{array}{r}1.6 \\
6.3 \\
3.0 \\
23.8 \\
21.0 \\
0.0 \\
18.9 \\
8.2 \\
17.2 \\
0.0\end{array}$ & $\begin{array}{r}1.6 \\
9.6 \\
4.0 \\
20.5 \\
30.6 \\
0.0 \\
12.3 \\
9.0 \\
12.0 \\
0.0\end{array}$ & $\begin{array}{r}4.1 \\
10.7 \\
0.9 \\
23.2 \\
29.5 \\
0.0 \\
9.6 \\
10.7 \\
7.4 \\
0.0\end{array}$ & $\begin{array}{r}4.4 \\
10.7 \\
5.2 \\
30.9 \\
26.5 \\
0.0 \\
6.2 \\
6.7 \\
5.5 \\
0.0\end{array}$ & $\begin{array}{r}4.6 \\
13.7 \\
4.1 \\
31.4 \\
25.7 \\
0.0 \\
6.8 \\
9.8 \\
3.8 \\
0.0\end{array}$ & 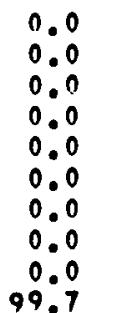 & $\begin{array}{l}0.0 \\
0.0 \\
0.0 \\
0.0 \\
0.0 \\
0.0 \\
0.0 \\
0.0 \\
0.0 \\
090\end{array}$ & $\begin{array}{l}0.0 \\
0.0 \\
0.0 \\
0.0 \\
0.0 \\
0.0 \\
0.0 \\
0.0 \\
0.0 \\
0.7\end{array}$ & $\begin{array}{r}0.0 \\
0.0 \\
0.0 \\
0.0 \\
0.0 \\
0.0 \\
0.0 \\
0.0 \\
0.0 \\
99.7\end{array}$ & $\begin{array}{r}0.0 \\
0.0 \\
0.0 \\
0.0 \\
0.0 \\
0.0 \\
0.0 \\
0.0 \\
0.0 \\
99.7\end{array}$ & $\begin{array}{l}0.0 \\
0.0 \\
0.0 \\
0.0 \\
0.0 \\
0.0 \\
0.0 \\
0.0 \\
0.0 \\
9.7\end{array}$ & $\begin{array}{r}0.0 \\
0.0 \\
0.0 \\
0.0 \\
0.0 \\
0.0 \\
0.0 \\
0.0 \\
0.0 \\
9.0 \\
9.7\end{array}$ \\
\hline
\end{tabular}

48 Dogrees North Latitude

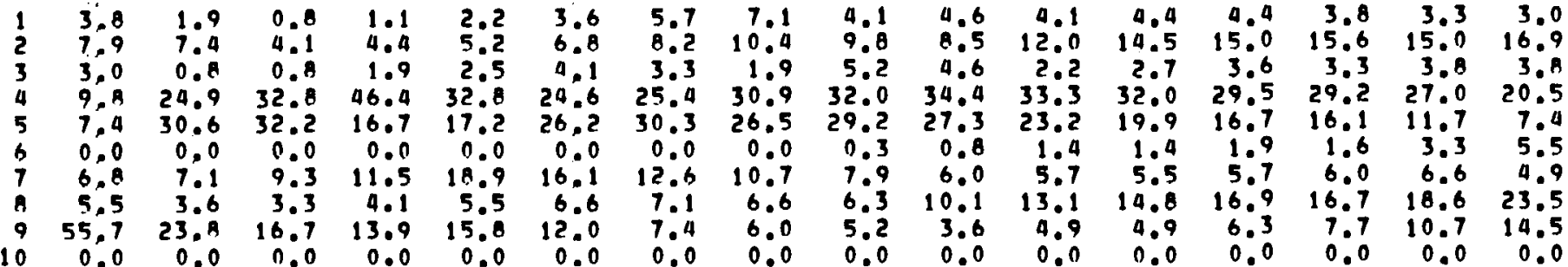

45 Degrese North Latltude

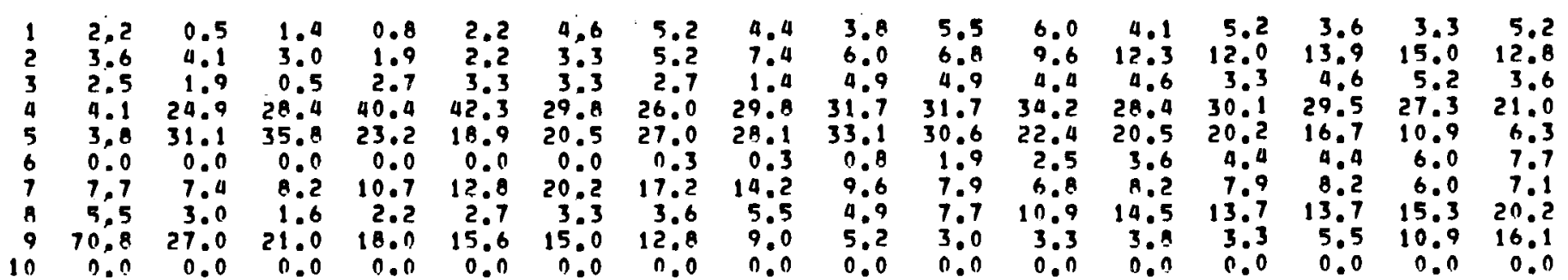

42 Deareos North Lotitude

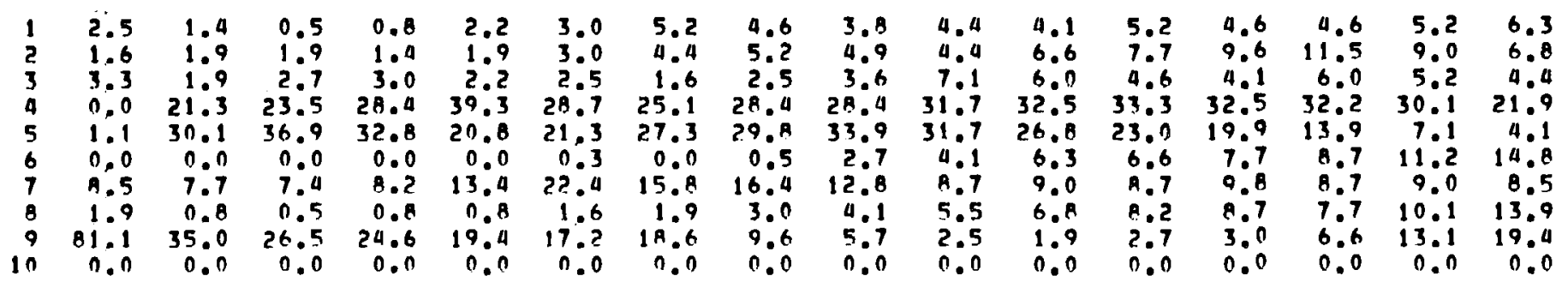


1976

39 Dearees North Lett tude

\begin{tabular}{|c|c|c|c|c|c|c|c|c|c|c|c|c|c|c|c|c|c|}
\hline$P T$ & 126 & 122 & 118 & 114 & 110 & 106 & $\begin{array}{l}\text { Deg } \\
102\end{array}$ & $\begin{array}{l}W \\
98\end{array}$ & & 94 & $\begin{array}{c}\text { tut de } \\
90\end{array}$ & 86 & 82 & 78 & 74 & 70 & 66 \\
\hline $\begin{array}{l}1 \\
2 \\
3 \\
4 \\
5 \\
6 \\
7 \\
8 \\
9 \\
10\end{array}$ & $\begin{array}{r}0.5 \\
0.3 \\
1.9 \\
0.0 \\
0.3 \\
0.0 \\
5.5 \\
1.4 \\
90.2 \\
0.0\end{array}$ & $\begin{array}{r}0.3 \\
0.5 \\
2.2 \\
12.3 \\
18.0 \\
0.0 \\
4.9 \\
0.0 \\
61.7 \\
0.0\end{array}$ & $\begin{array}{r}0.3 \\
0.8 \\
2.2 \\
17.5 \\
37.2 \\
0.0 \\
4.9 \\
0.5 \\
36.6 \\
0.0\end{array}$ & $\begin{array}{r}1.4 \\
0.8 \\
1.4 \\
15.3 \\
38.8 \\
0.0 \\
7.1 \\
0.0 \\
32.0 \\
0.0\end{array}$ & $\begin{array}{r}1.6 \\
1.1 \\
1.4 \\
29.5 \\
29.2 \\
0.0 \\
9.3 \\
0.8 \\
27.0 \\
0.0\end{array}$ & $\begin{array}{r}1.9 \\
1.9 \\
1.4 \\
31.1 \\
21.9 \\
0.3 \\
16.4 \\
0.0 \\
24.3 \\
0.0\end{array}$ & $\begin{array}{r}2.2 \\
3.0 \\
2.7 \\
19.4 \\
29.2 \\
1.4 \\
16.7 \\
0.8 \\
24.6 \\
0.0\end{array}$ & $\begin{array}{r}3.0 \\
3.6 \\
2.7 \\
27.0 \\
30.3 \\
6.3 \\
14.2 \\
1.4 \\
11.5 \\
0.0\end{array}$ & $\begin{array}{r}3 \\
27 \\
31 \\
10 \\
12 \\
3 \\
3 \\
0\end{array}$ & $\begin{array}{l}3.3 \\
.3 \\
.4 \\
0.4 \\
.0 \\
.3 \\
3.6 \\
0.0\end{array}$ & $\begin{array}{r}4.6 \\
4.6 \\
4.4 \\
30.3 \\
30.3 \\
10.9 \\
10.7 \\
2.7 \\
1.4 \\
0.0\end{array}$ & $\begin{array}{r}5.2 \\
5.7 \\
6.3 \\
30.9 \\
25.4 \\
10.1 \\
10.9 \\
3.3 \\
2.2 \\
0.0\end{array}$ & $\begin{array}{r}6.6 \\
7.9 \\
4.6 \\
35.0 \\
19.1 \\
12.6 \\
10.1 \\
1.4 \\
2.7 \\
0.0\end{array}$ & $\begin{array}{r}6.3 \\
6.5 \\
5.5 \\
34.4 \\
16.0 \\
11.5 \\
10.9 \\
3.0 \\
3.6 \\
0.0\end{array}$ & $\begin{array}{r}6.3 \\
5.5 \\
4.6 \\
35.8 \\
8.5 \\
16.7 \\
8.5 \\
4.4 \\
9.8 \\
0.0\end{array}$ & $\begin{array}{r}6.8 \\
3.8 \\
3.8 \\
20.2 \\
3.8 \\
20.2 \\
6.6 \\
7.4 \\
18.3 \\
0.0\end{array}$ & $\begin{array}{c}0.0 \\
0.0 \\
0.0 \\
0.0 \\
0.0 \\
0.0 \\
0.0 \\
0.0 \\
0.0 \\
99.7\end{array}$ \\
\hline
\end{tabular}

36 Dearees North Latitude

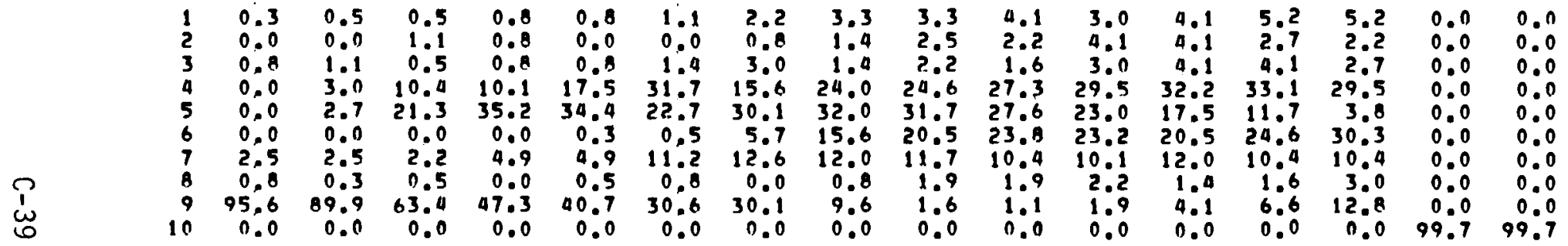

33 Dearees North Latitude

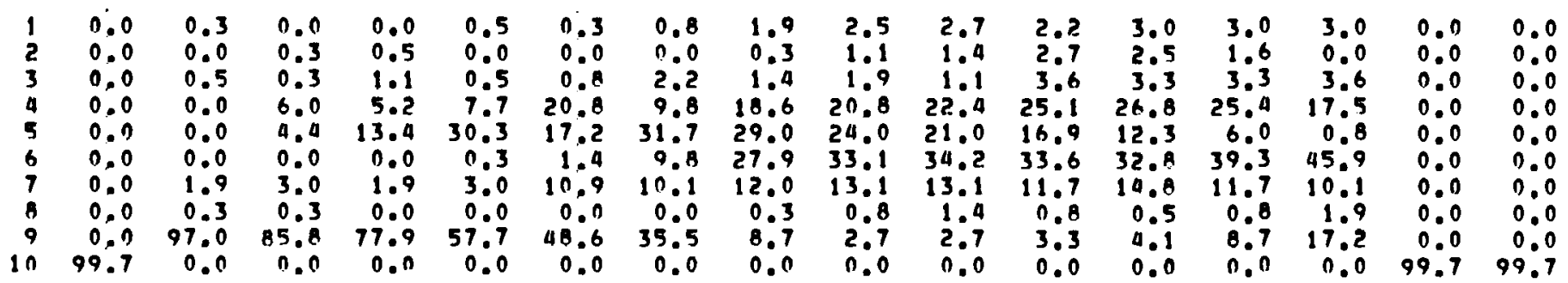

30 Dagrees Nopth Lat 1 tuda

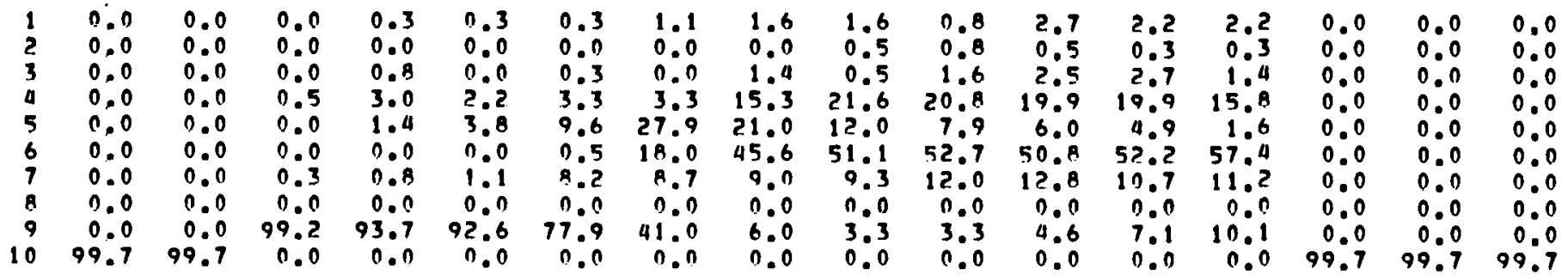


1976

27 Degrees Noreh Latltude

\begin{tabular}{|c|c|c|c|c|c|c|c|c|c|c|c|c|c|c|c|c|}
\hline WPT & 126 & 122 & 118 & 114 & 110 & 106 & $\begin{array}{l}\text { Dea } \\
102\end{array}$ & $\begin{array}{c}98 \\
98\end{array}$ & $\begin{array}{l}\text { at } \mathrm{Lor} \\
94\end{array}$ & $\begin{array}{c}\text { it tut de } \\
\text { on }\end{array}$ & 86 & B2 & 78 & 74 & 70 & 66 \\
\hline $\begin{array}{r}1 \\
2 \\
3 \\
a \\
5 \\
6 \\
7 \\
6 \\
9 \\
10\end{array}$ & $\begin{aligned} 0.0 \\
0.0 \\
0.0 \\
0.0 \\
0.0 \\
0.0 \\
0.0 \\
0.0 \\
0.0 \\
99.7\end{aligned}$ & $\begin{array}{r}0.0 \\
0.0 \\
0.0 \\
0.0 \\
0.0 \\
0.0 \\
0.0 \\
0.0 \\
0.0 \\
99.7\end{array}$ & $\begin{array}{c}0.0 \\
0.0 \\
0.0 \\
0.0 \\
0.0 \\
0.0 \\
0.0 \\
0.0 \\
0.0 \\
99.7\end{array}$ & $\begin{array}{l}0.0 \\
0.0 \\
0.0 \\
0.0 \\
0.0 \\
0.0 \\
0.0 \\
0.0 \\
0.0 \\
99.7\end{array}$ & $\begin{array}{r}0.0 \\
0.0 \\
0.0 \\
0.0 \\
0.0 \\
0.0 \\
0.0 \\
0.0 \\
0.0 \\
99.7\end{array}$ & $\begin{array}{c}0.0 \\
0.0 \\
0.0 \\
0.0 \\
0.0 \\
0.0 \\
0.0 \\
0.0 \\
0.0 \\
99.7\end{array}$ & $\begin{array}{r}0.5 \\
0.0 \\
0.3 \\
0.3 \\
15.6 \\
17.2 \\
7.4 \\
0.0 \\
58.7 \\
0.0\end{array}$ & $\begin{array}{r}0.0 \\
0.0 \\
0.8 \\
13.1 \\
13.9 \\
53.6 \\
7.1 \\
0.0 \\
11.5 \\
0.0\end{array}$ & $\begin{array}{r}0.5 \\
0.3 \\
0.5 \\
15.6 \\
5.5 \\
60.9 \\
10.9 \\
0.0 \\
6.3 \\
0.0\end{array}$ & $\begin{array}{r}0.6 \\
0.3 \\
1.9 \\
13.4 \\
4.1 \\
64.5 \\
9.3 \\
0.0 \\
5.7 \\
0.0\end{array}$ & $\begin{array}{r}0.8 \\
0.3 \\
1.4 \\
12.3 \\
2.5 \\
65.0 \\
10.1 \\
0.0 \\
7.7 \\
0.0\end{array}$ & $\begin{array}{r}0.8 \\
0.3 \\
1.4 \\
11.2 \\
1.1 \\
67.5 \\
9.6 \\
0.0 \\
0.2 \\
0.0\end{array}$ & $\begin{array}{r}0.8 \\
0.0 \\
1.4 \\
6.6 \\
0.3 \\
70.2 \\
10.7 \\
0.0 \\
10.1 \\
0.0\end{array}$ & $\begin{array}{rl}0 . & 0 \\
0.0 & 0 \\
0.0 & 0 \\
0.0 \\
0.0 \\
0.0 \\
0.0 \\
0.0 \\
0.0 \\
99.7\end{array}$ & $\begin{array}{rl}0.0 & 0 \\
0.0 & 0 \\
0.0 \\
0.0 \\
0.0 \\
0.0 \\
0.0 \\
0.0 \\
0.0 \\
99.7\end{array}$ & $\begin{array}{l}0.0 \\
0.0 \\
0.0 \\
0.0 \\
0.0 \\
0.0 \\
0.0 \\
0.0 \\
0.0 \\
99.7\end{array}$ \\
\hline
\end{tabular}

24 Degrees North Lotitude

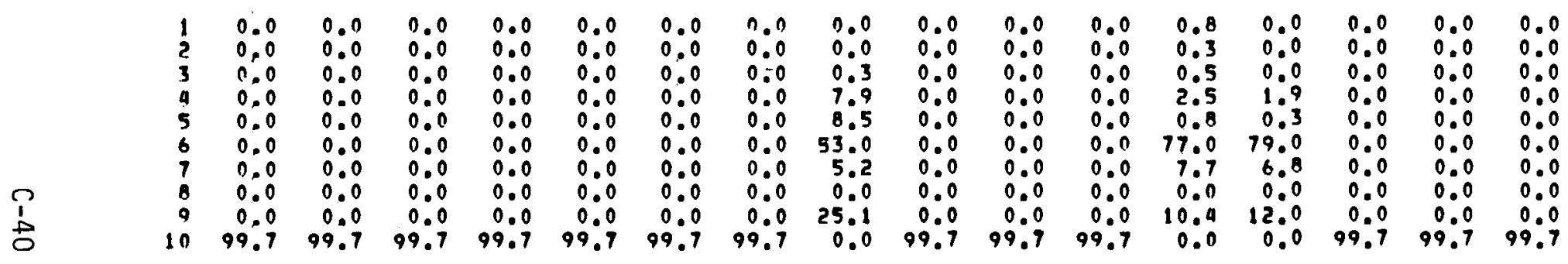


1977

51 Deorees North Lotitude

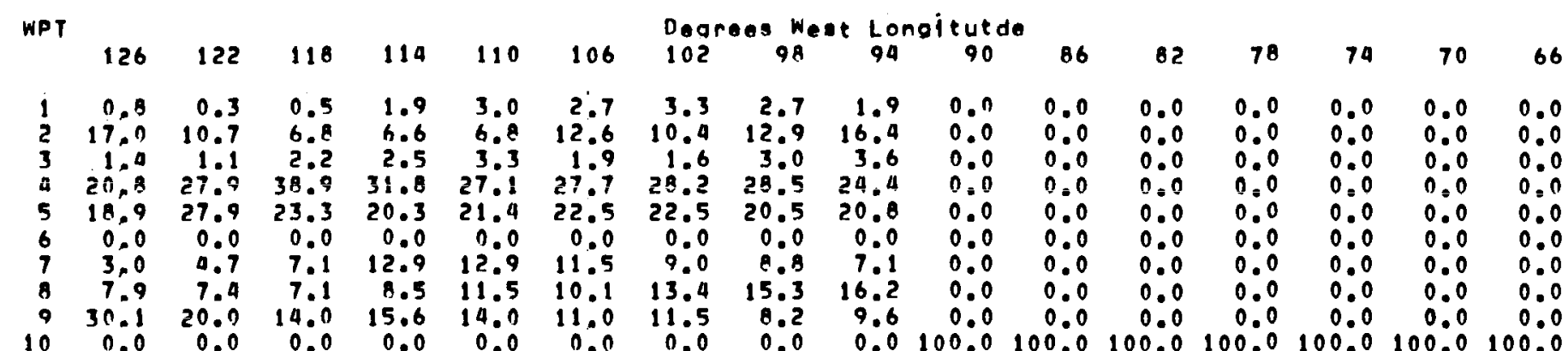

as Degreas North Letitude

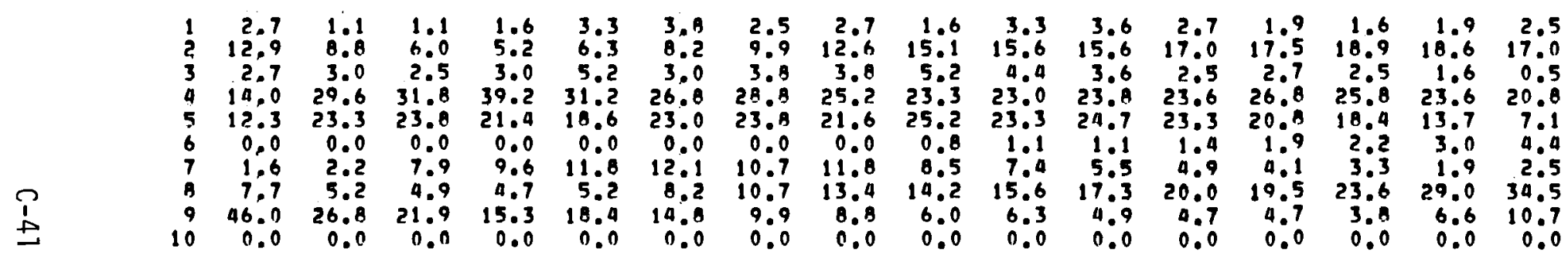

45 Deareas North Latitude

\begin{tabular}{|c|c|c|c|c|c|c|c|c|c|c|c|c|c|c|c|c|}
\hline $\begin{array}{c}1 \\
2 \\
3 \\
4 \\
5 \\
6 \\
7 \\
8 \\
9 \\
10\end{array}$ & $\begin{array}{r}2.5 \\
7.9 \\
4.7 \\
6.8 \\
6.0 \\
0.0 \\
3.0 \\
5.5 \\
63.6 \\
0.0\end{array}$ & $\begin{array}{r}1.6 \\
6.0 \\
3.6 \\
28.5 \\
21.6 \\
0.0 \\
3.8 \\
3.6 \\
31.2 \\
0.0\end{array}$ & $\begin{array}{r}0.5 \\
3.3 \\
5.2 \\
20.0 \\
26.0 \\
0.0 \\
6.3 \\
2.2 \\
27.7 \\
0.0\end{array}$ & $\begin{array}{r}1.1 \\
3.0 \\
3.8 \\
34.5 \\
21.6 \\
0.0 \\
9.9 \\
1.6 \\
24.4 \\
0.0\end{array}$ & $\begin{array}{r}2.7 \\
3.3 \\
4.9 \\
36.7 \\
17.8 \\
0.9 \\
13.4 \\
2.7 \\
18.9 \\
0.0\end{array}$ & $\begin{array}{r}3.3 \\
4.9 \\
4.7 \\
26.3 \\
18.9 \\
0.0 \\
19.5 \\
5.8 \\
16.7 \\
0.0\end{array}$ & $\begin{array}{r}5.5 \\
8.5 \\
3.8 \\
26.0 \\
23.0 \\
0.3 \\
15.1 \\
5.2 \\
12.6 \\
0.0\end{array}$ & $\begin{array}{r}3.6 \\
12.1 \\
3.3 \\
24.1 \\
23.6 \\
0.5 \\
15.3 \\
0.5 \\
9.0 \\
0.0\end{array}$ & $\begin{array}{r}2.7 \\
10.7 \\
5.2 \\
21.4 \\
27.4 \\
3.3 \\
11.2 \\
9.9 \\
6.2 \\
0.0\end{array}$ & $\begin{array}{r}4.1 \\
12.3 \\
4.1 \\
24.4 \\
24.1 \\
2.7 \\
11.0 \\
11.8 \\
5.5 \\
0.0\end{array}$ & $\begin{array}{r}5.5 \\
12.6 \\
4.7 \\
23.0 \\
23.6 \\
2.5 \\
9.3 \\
16.4 \\
2.5 \\
0.0\end{array}$ & $\begin{array}{r}4.1 \\
15.1 \\
3.8 \\
26.0 \\
21.9 \\
2.7 \\
8.2 \\
16.2 \\
1.9 \\
0.0\end{array}$ & $\begin{array}{r}1.9 \\
16.2 \\
5.2 \\
22.2 \\
21.6 \\
3.6 \\
6.6 \\
19.7 \\
3.0 \\
0.0\end{array}$ & $\begin{array}{r}3.6 \\
16.7 \\
2.2 \\
26.6 \\
17.3 \\
3.8 \\
6.0 \\
20.0 \\
3.8 \\
0.0\end{array}$ & $\begin{array}{r}3.0 \\
16.7 \\
2.7 \\
23.0 \\
12.9 \\
4.7 \\
4.9 \\
23.8 \\
8.2 \\
0.0\end{array}$ & $\begin{array}{r}2.7 \\
16.7 \\
3.8 \\
18.4 \\
7.1 \\
7.1 \\
4.4 \\
27.1 \\
12.6 \\
0.0\end{array}$ \\
\hline
\end{tabular}

42 Dearees North Latitude

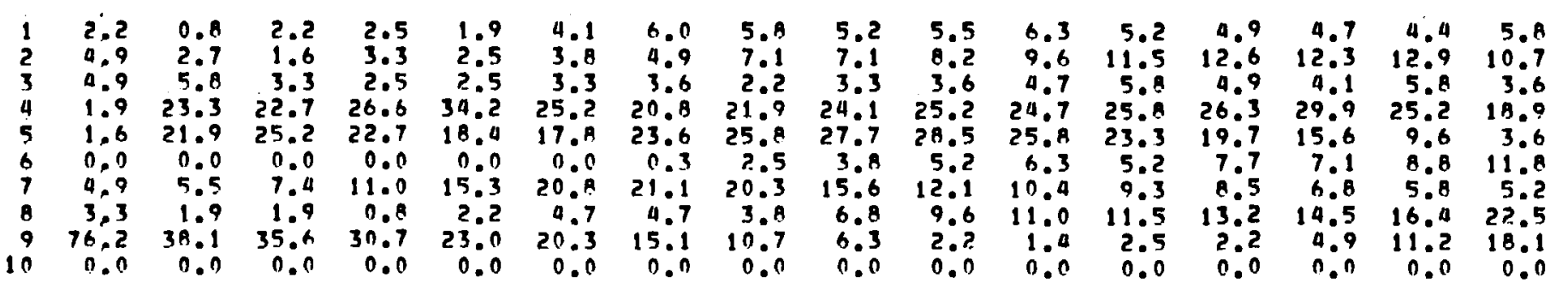


1977

39 Degrese North Latitude

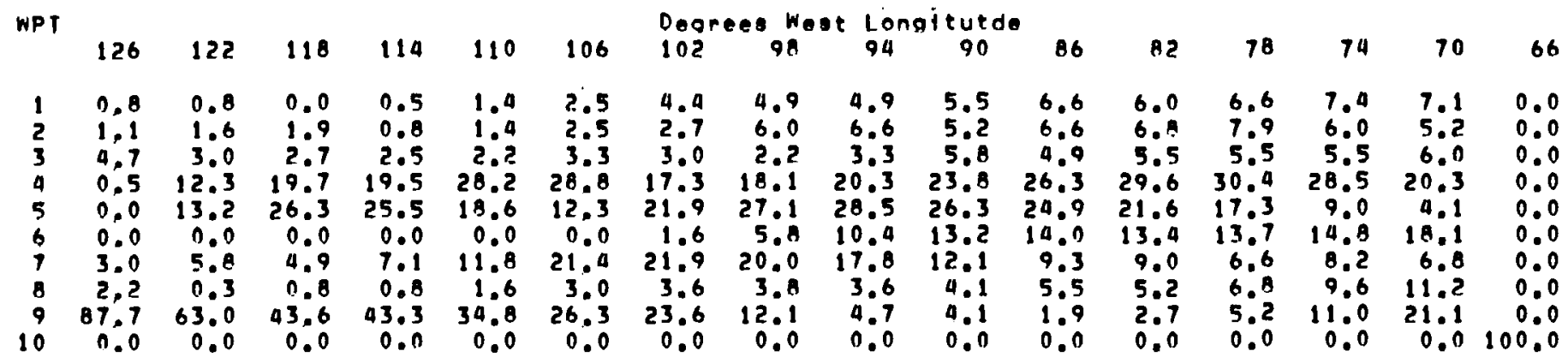

36 Degrees North Latitude

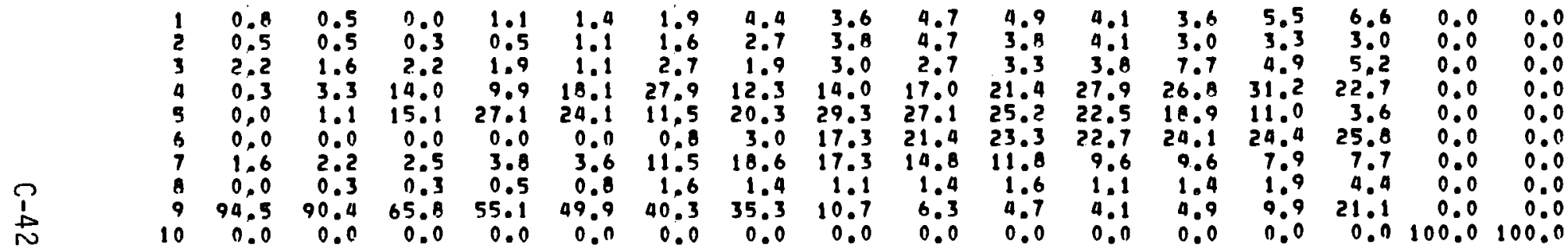

33 Dearees North Latltude

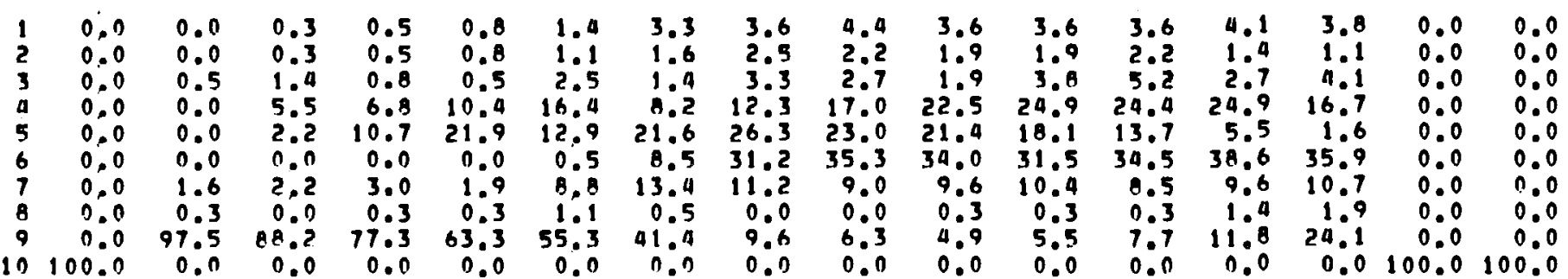

30 nearees North Latitude

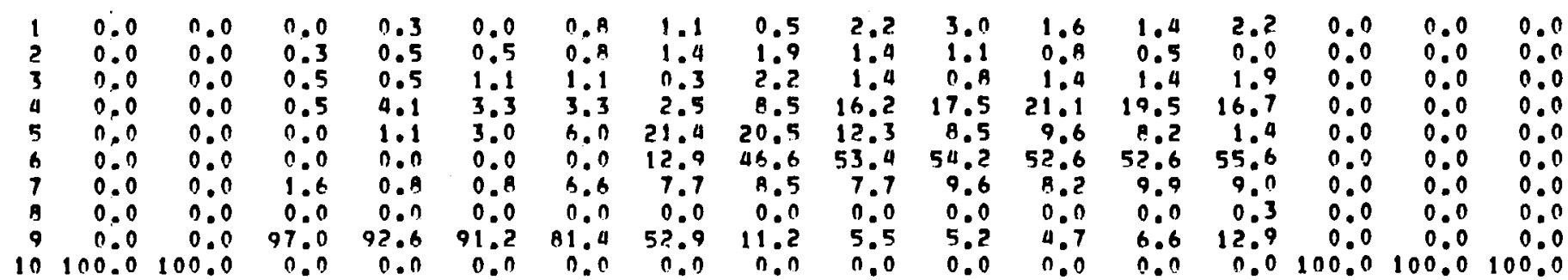


1977

27 Dearees North Let 1 tude

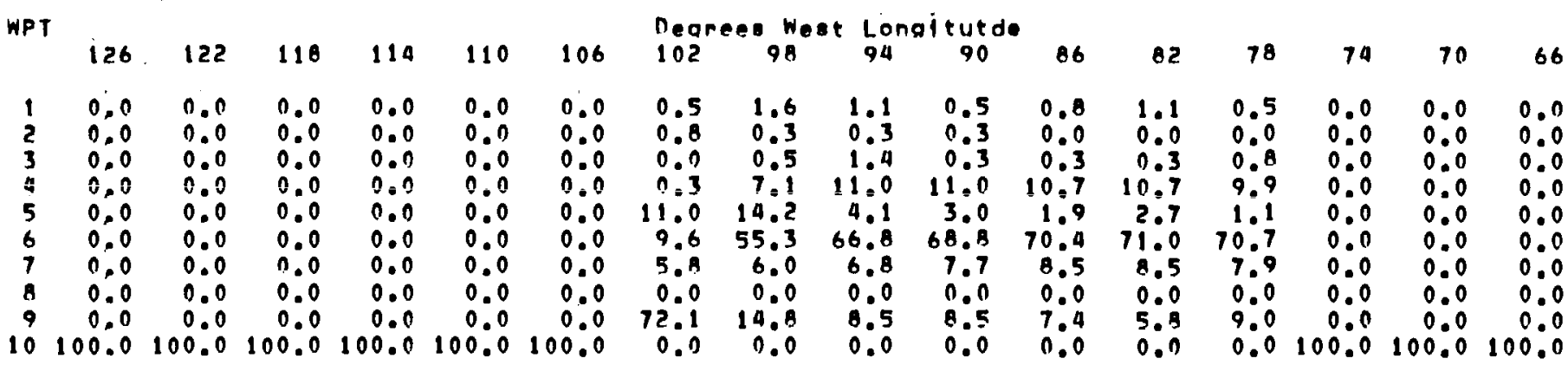

24 Degrees North Lat ftude

$\begin{array}{rrrrrrrrrrrrrrrrr}1 & 0.0 & 0.0 & 0.0 & 0.0 & 0.0 & 0.0 & 0.0 & 0.5 & 0.0 & 0.0 & 0.0 & 0.0 & 0.3 & 0.0 & 0.0 & 0.0 \\ 2 & 0.0 & 0.0 & 0.0 & 0.0 & 0.0 & 0.0 & 0.0 & 0.0 & 0.0 & 0.0 & 0.0 & 0.0 & 0.0 & 0.0 & 0.0 & 0.0 \\ 3 & 0.0 & 0.0 & 0.0 & 0.0 & 0.0 & 0.0 & 0.0 & 0.3 & 0.0 & 0.0 & 0.0 & 0.3 & 0.5 & 0.0 & 0.0 & 0.0 \\ 4 & 0.0 & 0.0 & 0.0 & 0.0 & 0.0 & 0.0 & 0.0 & 5.2 & 0.0 & 0.0 & 0.0 & 5.2 & 3.0 & 0.0 & 0.0 & 0.0 \\ 5 & 0.0 & 0.0 & 0.0 & 0.0 & 0.0 & 0.0 & 0.0 & 10.0 & 0.0 & 0.0 & 0.0 & 0.3 & 0.0 & 0.0 & 0.0 & 0.0 \\ 6 & 0.0 & 0.0 & 0.0 & 0.0 & 0.0 & 0.0 & 0.0 & 52.0 & 0.0 & 0.0 & 0.0 & 77.0 & 75.1 & 0.0 & 0.0 & 0.0 \\ 7 & 0.0 & 0.0 & 0.0 & 0.0 & 0.0 & 0.0 & 0.0 & 3.0 & 0.0 & 0.0 & 0.0 & 8.0 & 0.2 & 0.0 & 0.0 & 0.0 \\ 8 & 0.0 & 0.0 & 0.0 & 0.0 & 0.0 & 0.0 & 0.0 & 0.0 & 0.0 & 0.0 & 0.0 & 0.0 & 0.0 & 0.0 & 0.0 & 0.0 \\ 9 & 0.0 & 0.0 & 0.0 & 0.0 & 0.0 & 0.0 & 0.0 & 27.1 & 0.0 & 0.0 & 0.0 & 9.0 & 12.1 & 0.0 & 0.0 & 0.0 \\ 10 & 100.0 & 100.0 & 100.0 & 100.0 & 100.0 & 100.0 & 100.0 & 0.3 & 100.0 & 100.0 & 100.0 & 0.0 & 0.0 & 100.0 & 100.0 & 100.0\end{array}$


1978

51 Dearaea North Lat ltude

\begin{tabular}{|c|c|c|c|c|c|c|c|c|c|c|c|c|c|c|c|c|}
\hline WPT & 126 & 122 & 118 & 114 & 110 & 106 & $\begin{array}{l}\text { Den } \\
102\end{array}$ & $\begin{array}{c}08 \\
9 B\end{array}$ & $\begin{array}{l}\text { Lor } \\
94\end{array}$ & $\begin{array}{l}\text { tutde } \\
90\end{array}$ & 86 & 82 & 78 & 74 & 70 & 66 \\
\hline $\begin{array}{c}1 \\
2 \\
3 \\
4 \\
5 \\
6 \\
7 \\
8 \\
9 \\
10\end{array}$ & $\begin{array}{r}0.0 \\
17.8 \\
1.6 \\
22.5 \\
14.8 \\
0.0 \\
4.9 \\
5.8 \\
31.8 \\
0.0\end{array}$ & $\begin{array}{r}1.6 \\
10.9 \\
1.4 \\
25.5 \\
20.5 \\
0.0 \\
8.5 \\
7.9 \\
24.1 \\
0.0\end{array}$ & $\begin{array}{r}1.6 \\
6.6 \\
3.8 \\
29.6 \\
18.4 \\
0.0 \\
16.2 \\
7.7 \\
16.2 \\
0.0\end{array}$ & $\begin{array}{r}2.7 \\
5.8 \\
3.0 \\
28.2 \\
18.4 \\
0.0 \\
17.8 \\
7.9 \\
16.2 \\
0.0\end{array}$ & $\begin{array}{r}3.6 \\
8.6 \\
2.7 \\
29.4 \\
20.7 \\
0.0 \\
12.3 \\
9.6 \\
19.0 \\
0.0\end{array}$ & $\begin{array}{r}3.0 \\
11.2 \\
3.3 \\
24.7 \\
23.8 \\
0.0 \\
9.0 \\
11.0 \\
14.0 \\
0.0\end{array}$ & $\begin{array}{r}4.4 \\
11.0 \\
4.4 \\
24.1 \\
24.4 \\
0.0 \\
10.7 \\
11.0 \\
10.1 \\
0.0\end{array}$ & $\begin{array}{r}4.9 \\
11.5 \\
4.4 \\
26.0 \\
24.7 \\
0.0 \\
7.7 \\
12.9 \\
7.9 \\
0.0\end{array}$ & $\begin{array}{r}5.2 \\
15.6 \\
4.1 \\
24.9 \\
27.4 \\
0.0 \\
6.8 \\
9.3 \\
6.6 \\
0.0\end{array}$ & $\begin{array}{l}0.0 \\
0.0 \\
0.0 \\
0.0 \\
0.0 \\
0.0 \\
0.0 \\
0.0 \\
0.0 \\
0.0\end{array}$ & $\begin{array}{lll}0 & 0 & 0 \\
0 & 0 & 0 \\
0 & 0 & 0 \\
0 & 0 & 0 \\
0 & 0 & 0 \\
0 & 0 & 0 \\
0 & 0 \\
0 & 0 \\
0.0 & 0 \\
0 & 0\end{array}$ & $\begin{array}{l}0.0 \\
0.0 \\
0.0 \\
0.0 \\
0.0 \\
0.0 \\
0.0 \\
0.0 \\
0.0 \\
0.0\end{array}$ & $\begin{array}{l}0.0 \\
0.0 \\
0.0 \\
0.0 \\
0.0 \\
0.0 \\
0.0 \\
0.0 \\
0.0 \\
0.0\end{array}$ & $\begin{array}{l}0.0 \\
0.0 \\
0.0 \\
0.0 \\
0.0 \\
0.0 \\
0.0 \\
0.0 \\
0.0 \\
0.0\end{array}$ & $\begin{array}{l}0.0 \\
0.0 \\
0.0 \\
0.0 \\
0.0 \\
0.0 \\
0.0 \\
0.0 \\
0.0 \\
0.0\end{array}$ & $\begin{array}{l}0.0 \\
0.0 \\
0.0 \\
0.0 \\
0.0 \\
0.0 \\
0.0 \\
0.0\end{array}$ \\
\hline
\end{tabular}

48 Degree North Lettude

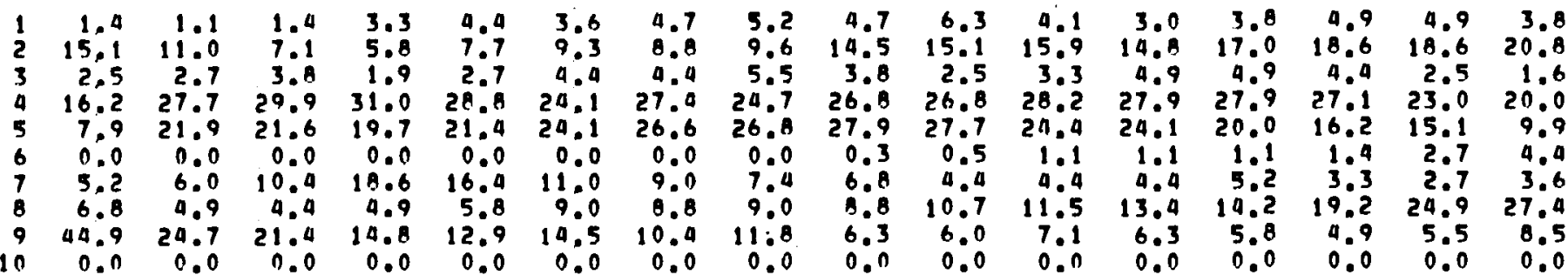

$\stackrel{p}{1}$

45 Dearees North Lettude

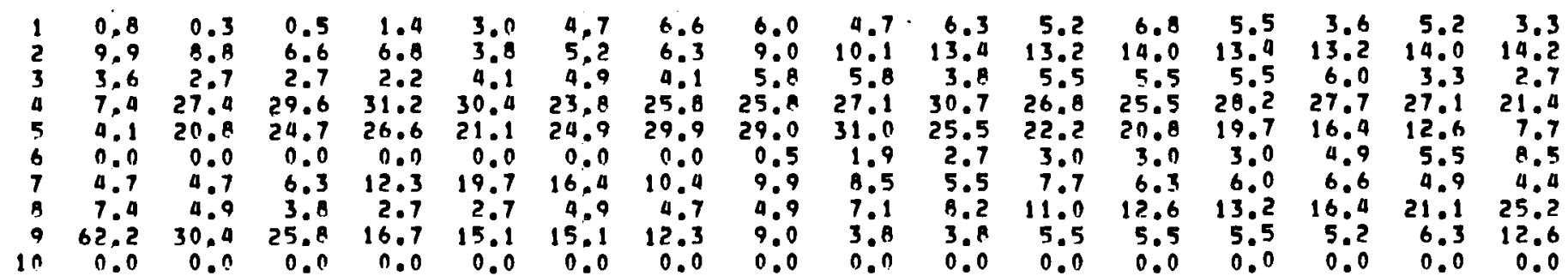

42 Degrees Nopth Lot Itude

\begin{tabular}{|c|c|c|c|c|c|c|c|c|c|c|c|c|c|c|c|c|}
\hline $\begin{array}{l}1 \\
2 \\
3 \\
4 \\
5 \\
6 \\
7 \\
9 \\
9 \\
10\end{array}$ & $\begin{array}{r}1.1 \\
7.1 \\
4.4 \\
1.6 \\
2.2 \\
0.0 \\
3.0 \\
0.0 \\
75.3 \\
0.0\end{array}$ & $\begin{array}{r}1.1 \\
6.6 \\
3.0 \\
23.6 \\
20.3 \\
0.0 \\
3.3 \\
3.3 \\
38.0 \\
0.0\end{array}$ & $\begin{array}{r}0.3 \\
4.9 \\
1.9 \\
27.4 \\
24.9 \\
0.0 \\
4.7 \\
3.3 \\
32.6 \\
0.0\end{array}$ & $\begin{array}{r}0.8 \\
6.6 \\
2.7 \\
25.5 \\
25.5 \\
0.0 \\
0.2 \\
2.5 \\
28.2 \\
0.0\end{array}$ & $\begin{array}{r}3.3 \\
5.8 \\
2.7 \\
29.3 \\
21.9 \\
0.0 \\
15.6 \\
1.6 \\
19.7 \\
0.0\end{array}$ & $\begin{array}{r}4.1 \\
6.0 \\
4.9 \\
25.5 \\
21.1 \\
0.0 \\
19.2 \\
1.9 \\
17.3 \\
0.0\end{array}$ & $\begin{array}{r}5.2 \\
5.8 \\
3.0 \\
20.5 \\
30.7 \\
0.0 \\
17.0 \\
2.2 \\
15.6 \\
0.0\end{array}$ & $\begin{array}{r}4.1 \\
7.4 \\
5.5 \\
26.3 \\
31.0 \\
1.6 \\
12.1 \\
2.7 \\
9.3 \\
0.0\end{array}$ & $\begin{array}{r}6.6 \\
7.7 \\
4.9 \\
27.7 \\
31.5 \\
0.1 \\
10.7 \\
3.8 \\
3.0 \\
0.0\end{array}$ & $\begin{array}{r}6.6 \\
9.6 \\
4.9 \\
30.1 \\
26.8 \\
6.3 \\
7.1 \\
5.8 \\
2.7 \\
0.0\end{array}$ & $\begin{array}{r}6.0 \\
12.6 \\
4.7 \\
30.1 \\
22.2 \\
5.2 \\
7.1 \\
7.9 \\
4.1 \\
0.0\end{array}$ & $\begin{array}{r}6.0 \\
12.9 \\
5.2 \\
27.9 \\
22.2 \\
4.7 \\
7.0 \\
9.0 \\
4.7 \\
0.0\end{array}$ & $\begin{array}{r}5.5 \\
12.1 \\
4.4 \\
31.2 \\
20.5 \\
6.0 \\
7.4 \\
9.6 \\
3.3 \\
0.0\end{array}$ & $\begin{array}{r}6.6 \\
11.5 \\
3.8 \\
34.8 \\
13.2 \\
6.6 \\
7.4 \\
9.9 \\
6.3 \\
0.0\end{array}$ & $\begin{array}{r}5.2 \\
9.9 \\
4.7 \\
29.9 \\
6.8 \\
9.6 \\
7.1 \\
15.6 \\
11.2 \\
0.0\end{array}$ & $\begin{array}{r}6.3 \\
10.4 \\
4.1 \\
19.7 \\
2.5 \\
11.2 \\
5.5 \\
22.2 \\
18.1 \\
0.0\end{array}$ \\
\hline
\end{tabular}


1978

39 Dearees North Latitude

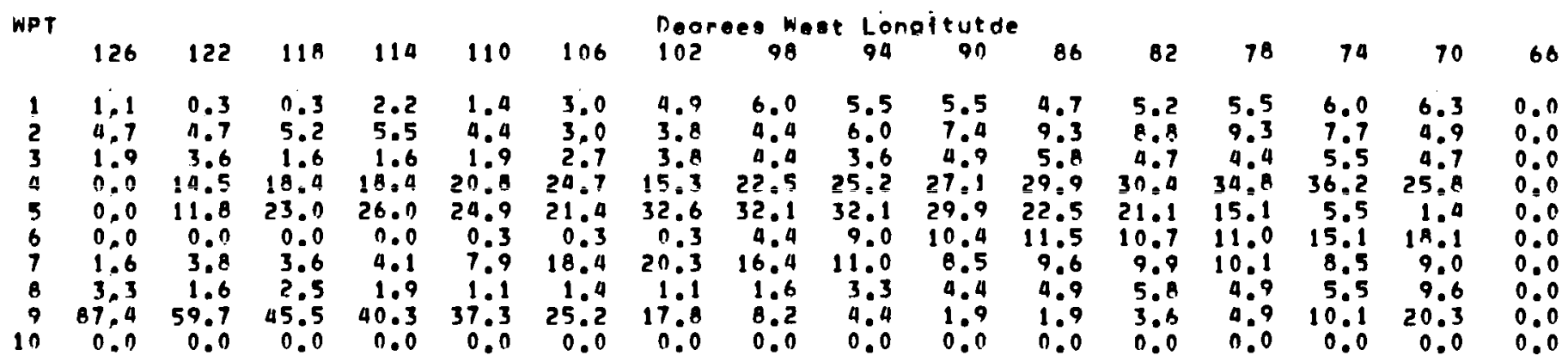

36 Degrees North Letftude



33 Deareos North Let itude

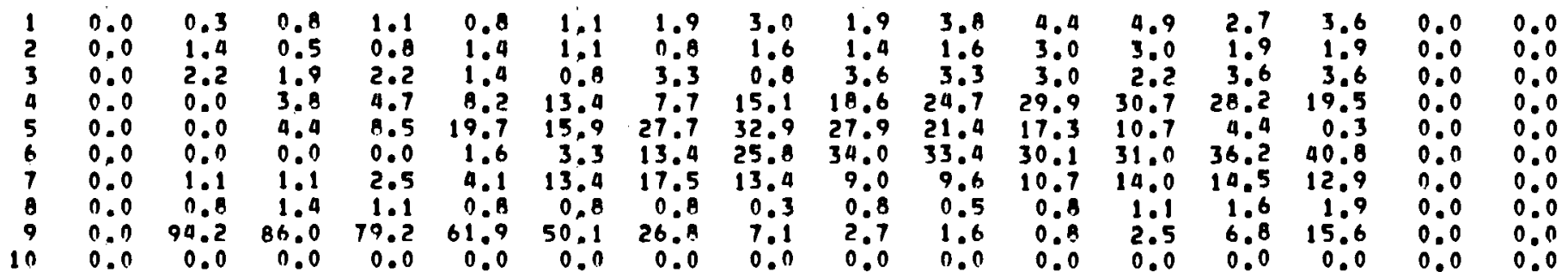

30 Degreas North Latleude

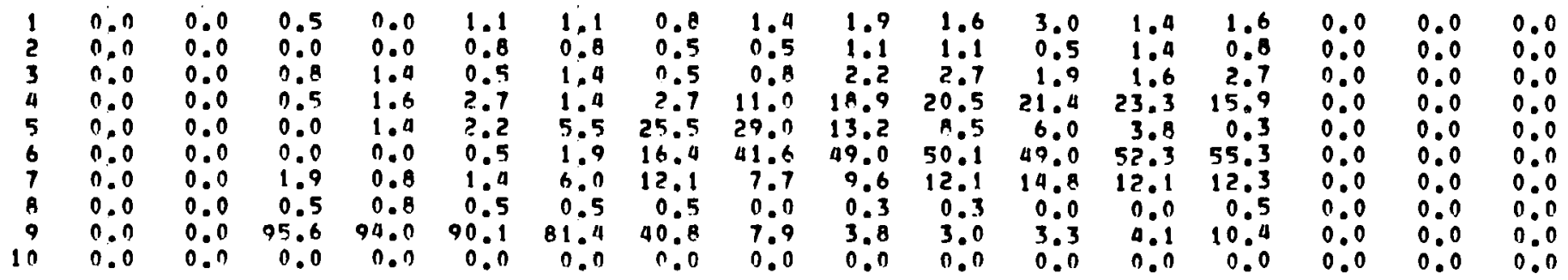


1978

27 Degrees Noreh Lotitude

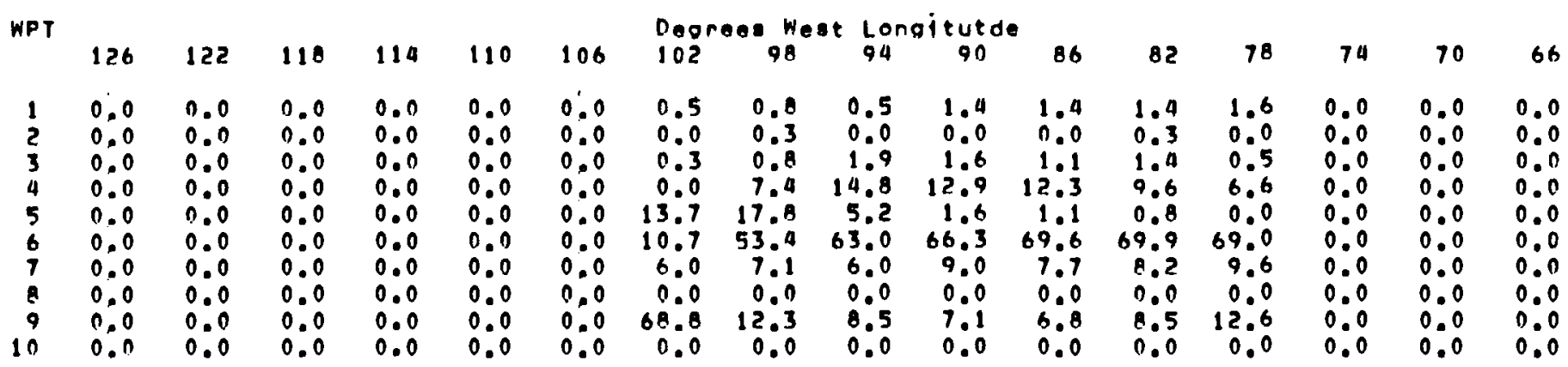

24 Depreas North Lotitude

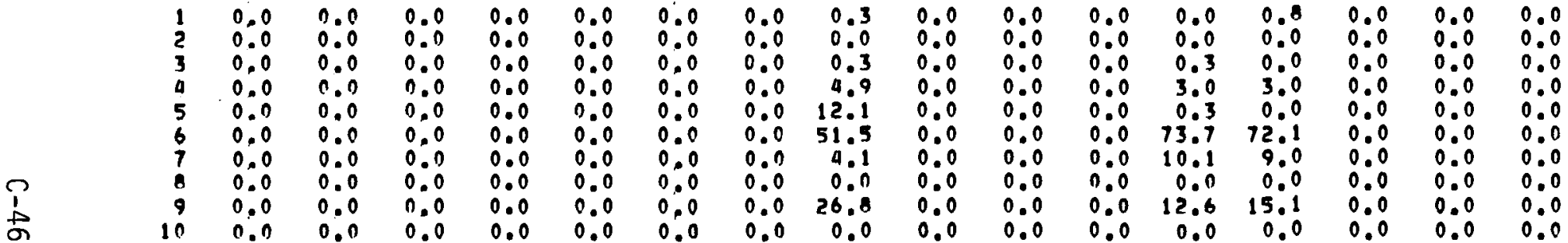


Voriability

51 Deareno North Latitude

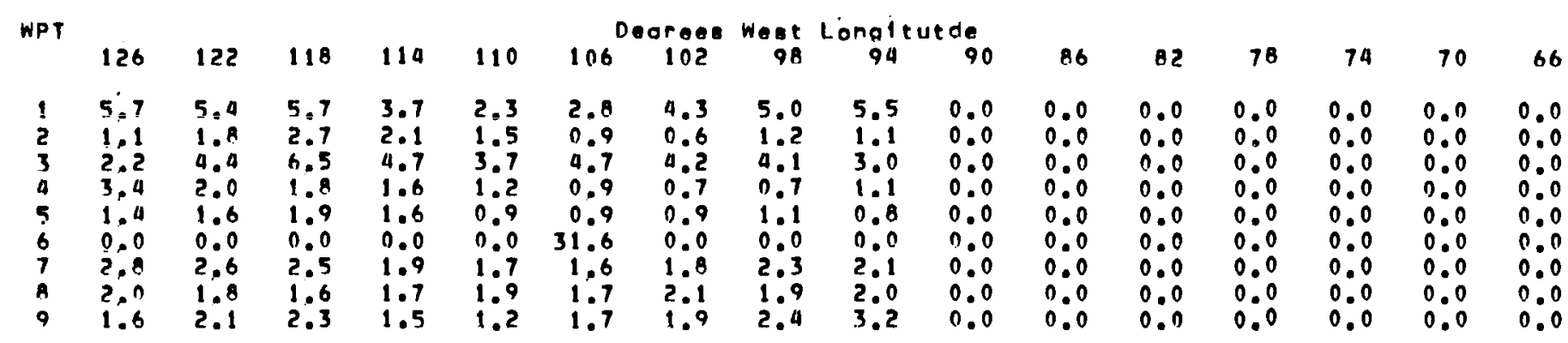

ab Doprese Noreh Latitude

$n$
1

$\begin{array}{rrrrrrrrrr}2.5 & 3.2 & 3.1 & 2.5 & 2.1 & 2.0 & 2.2 & 3.0 & 2.4 & 3.2 \\ 1.6 & 1.2 & 1.4 & 1.9 & 1.2 & 1.0 & 1.5 & 2.2 & 1.9 & 2.0 \\ 1.8 & 3.5 & 2.9 & 3.2 & 2.8 & 3.6 & 3.7 & 2.9 & 4.3 & 4.9 \\ 0.7 & 0.9 & 1.1 & 1.1 & 1.0 & 1.0 & 0.6 & 0.7 & 0.8 & 0.5 \\ 0.9 & 0.9 & 0.6 & 0.9 & 0.8 & 1.0 & 1.4 & 1.3 & 1.2 & 2.5 \\ 0.0 & 21.1 & 10.5 & 7.6 & 6.1 & 3.4 & 4.2 & 2.8 & 2.1 & 2.6 \\ 1.5 & 2.0 & 1.6 & 2.4 & 3.0 & 2.1 & 1.7 & 3.6 & 4.7 & 4.1 \\ 2.2 & 2.4 & 2.4 & 2.4 & 2.0 & 1.8 & 2.0 & 1.9 & 1.9 & 2.0 \\ 2.4 & 2.3 & 2.6 & 3.2 & 2.8 & 2.8 & 3.0 & 3.3 & 2.7 & 2.5\end{array}$

45 Dooreses North Letitude

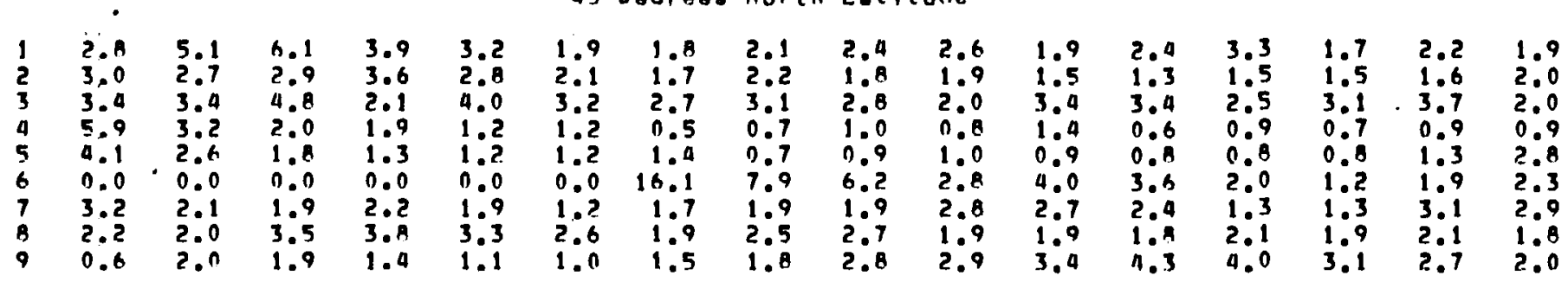

42 Deprees North Lotitude

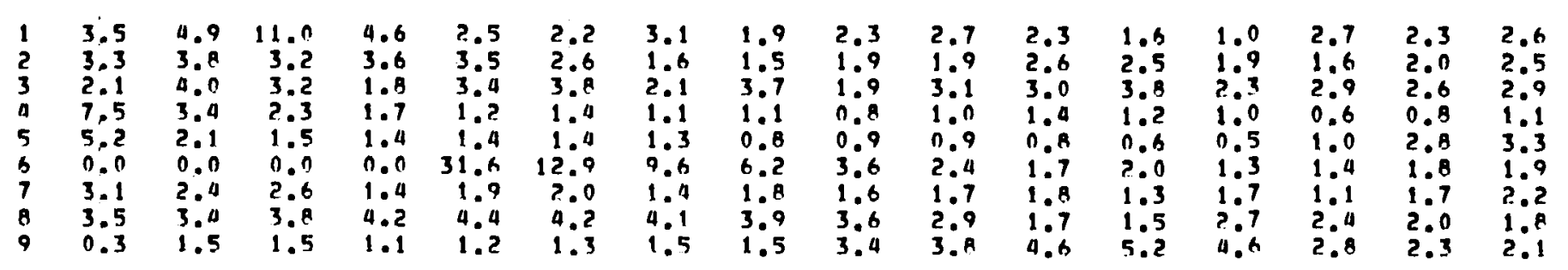


variability

39 Deareos North Letitude

\begin{tabular}{|c|c|c|c|c|c|c|c|c|c|c|c|c|c|c|c|c|}
\hline WPT & 126 & 122 & 118 & 114 & 110 & $106^{\circ}$ & $\begin{array}{l}\text { areas } \\
102\end{array}$ & $\begin{array}{r}\text { WeOt } \\
98\end{array}$ & $\begin{array}{c}\text { Longlet } \\
94\end{array}$ & tde & 86 & 82 & 78 & 74 & 70 & 66 \\
\hline $\begin{array}{l}1 \\
2 \\
3 \\
0 \\
5 \\
6 \\
7 \\
8 \\
9\end{array}$ & $\begin{array}{r}7.0 \\
6.7 \\
3.0 \\
11.8 \\
11.0 \\
0.0 \\
3.3 \\
3.6 \\
0.3\end{array}$ & $\begin{array}{r}10.2 \\
5.3 \\
2.6 \\
3.9 \\
2.0 \\
0.0 \\
1.7 \\
5.4 \\
0.0\end{array}$ & $\begin{array}{l}A .3 \\
5.0 \\
2.4 \\
2.8 \\
1.6 \\
0.0 \\
2.0 \\
4.5 \\
1.0\end{array}$ & $\begin{array}{l}4.1 \\
4.9 \\
3.3 \\
1.9 \\
1.4 \\
0.0 \\
1.9 \\
5.3 \\
1.0\end{array}$ & $\begin{array}{r}3.6 \\
4.0 \\
2.8 \\
1.7 \\
1.8 \\
21.1 \\
3.0 \\
5.0 \\
1.4\end{array}$ & $\begin{array}{r}3.6 \\
3.5 \\
3.3 \\
1.5 \\
2.0 \\
14.3 \\
2.1 \\
4.4 \\
1.2\end{array}$ & $\begin{array}{l}3.2 \\
2.9 \\
2.6 \\
1.4 \\
1.7 \\
6.3 \\
1.3 \\
6.0 \\
1.2\end{array}$ & $\begin{array}{l}2.5 \\
3.1 \\
3.9 \\
1.2 \\
1.1 \\
3.3 \\
1.4 \\
5.1 \\
1.6\end{array}$ & $\begin{array}{l}2.4 \\
1.7 \\
1.5 \\
0.9 \\
0.9 \\
1.2 \\
1.6 \\
4.5 \\
3.0\end{array}$ & $\begin{array}{l}1.7 \\
1.8 \\
1.1 \\
1.0 \\
1.1 \\
1.3 \\
1.3 \\
4.9 \\
3.8\end{array}$ & $\begin{array}{l}1.9 \\
2.0 \\
2.9 \\
1.1 \\
0.9 \\
1.5 \\
1.0 \\
3.0 \\
4.5\end{array}$ & $\begin{array}{l}2.0 \\
1.9 \\
3.2 \\
1.3 \\
0.8 \\
1.4 \\
1.1 \\
3.1 \\
3.2\end{array}$ & $\begin{array}{l}2.5 \\
2.0 \\
3.0 \\
0.8 \\
0.8 \\
1.5 \\
1.7 \\
2.3 \\
3.2\end{array}$ & $\begin{array}{l}2.6 \\
2.1 \\
2.6 \\
0.9 \\
2.9 \\
1.1 \\
1.4 \\
2.9 \\
2.4\end{array}$ & $\begin{array}{l}2.3 \\
1.5 \\
2.3 \\
1.3 \\
0.9 \\
0.9 \\
1.9 \\
2.1 \\
1.5\end{array}$ & $\begin{array}{l}0.0 \\
0.0 \\
0.0 \\
0.0 \\
0.0 \\
0.0 \\
0.0 \\
0.0 \\
0.00 \\
0.0\end{array}$ \\
\hline
\end{tabular}

36 Degrees North Latitude

\begin{tabular}{|c|c|c|c|c|c|c|c|c|c|c|c|c|c|c|c|c|c|}
\hline$?$ & $\begin{array}{l}1 \\
2 \\
3 \\
4 \\
5 \\
6 \\
7 \\
8 \\
9\end{array}$ & $\begin{array}{r}6.3 \\
12.4 \\
4.6 \\
16.1 \\
16.1 \\
0.0 \\
4.9 \\
5.9 \\
0.3\end{array}$ & $\begin{array}{l}9.3 \\
8.7 \\
4.4 \\
4.0 \\
3.4 \\
0.0 \\
2.1 \\
5.2 \\
0.2\end{array}$ & $\begin{array}{l}7.4 \\
5.6 \\
4.1 \\
3.5 \\
1.8 \\
0.0 \\
4.3 \\
4.2 \\
0.6\end{array}$ & $\begin{array}{l}4.9 \\
3.3 \\
3.3 \\
2.9 \\
1.3 \\
0.0 \\
2.2 \\
5.0 \\
0.9\end{array}$ & $\begin{array}{r}4.5 \\
5.5 \\
3.5 \\
2.1 \\
1.5 \\
11.7 \\
2.3 \\
4.9 \\
0.9\end{array}$ & $\begin{array}{l}4.9 \\
5.0 \\
3.4 \\
1.5 \\
2.1 \\
6.7 \\
1.9 \\
3.9 \\
0.9\end{array}$ & $\begin{array}{l}2.9 \\
0.8 \\
3.8 \\
1.6 \\
1.9 \\
3.0 \\
1.8 \\
7.0 \\
1.0\end{array}$ & $\begin{array}{l}2.5 \\
3.6 \\
3.7 \\
1.8 \\
1.2 \\
1.4 \\
1.5 \\
6.9 \\
1.7\end{array}$ & $\begin{array}{l}3.0 \\
2.2 \\
3.7 \\
0.9 \\
1.3 \\
1.3 \\
1.1 \\
6.0 \\
3.3\end{array}$ & $\begin{array}{l}1.3 \\
3.4 \\
4.6 \\
1.0 \\
1.6 \\
1.4 \\
2.0 \\
5.4 \\
3.2\end{array}$ & $\begin{array}{l}1.8 \\
2.0 \\
2.9 \\
1.9 \\
1.6 \\
1.9 \\
1.0 \\
9.7 \\
3.2\end{array}$ & $\begin{array}{l}1.7 \\
2.2 \\
4.0 \\
1.5 \\
1.2 \\
1.3 \\
1.7 \\
4.3 \\
2.7\end{array}$ & $\begin{array}{l}1.6 \\
2.7 \\
1.5 \\
1.2 \\
3.3 \\
1.0 \\
1.9 \\
2.7 \\
3.2\end{array}$ & $\begin{array}{l}1.9 \\
2.6 \\
2.1 \\
1.5 \\
4.4 \\
0.9 \\
2.4 \\
3.1 \\
2.4\end{array}$ & $\begin{array}{ll}0 & 0 \\
0.0 & 0 \\
0.0 & 0 \\
0.0 & 0 \\
0.0 & 0 \\
0.0 & 0 \\
0.0 & 0 \\
0.0 & 0 \\
0.0 & 0\end{array}$ & 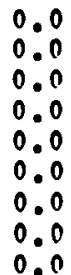 \\
\hline
\end{tabular}

33 Denrees North Latitude

\begin{tabular}{|c|c|c|c|c|c|c|c|c|c|c|c|c|c|c|c|c|}
\hline 1 & $\begin{array}{ll}0 & 0 \\
0 & 0 \\
0.0 & 0 \\
0.0 & 0 \\
0.0 & 0 \\
0.0 & 0 \\
0.0 & 0 \\
0.0 & 0 \\
0.0 & 0\end{array}$ & $\begin{array}{r}14.8 \\
14.5 \\
6.6 \\
17.5 \\
17.5 \\
0.0 \\
2.8 \\
6.5 \\
0.1\end{array}$ & $\begin{array}{l}6.9 \\
7.3 \\
6.8 \\
4.7 \\
2.7 \\
0.0 \\
2.9 \\
8.6 \\
0.3\end{array}$ & $\begin{array}{r}6.5 \\
6.2 \\
5.3 \\
3.4 \\
2.1 \\
31.6 \\
2.5 \\
7.8 \\
0.5\end{array}$ & $\begin{array}{r}6.1 \\
8.0 \\
4.4 \\
2.1 \\
1.3 \\
23.4 \\
3.3 \\
5.3 \\
0.6\end{array}$ & $\begin{array}{l}6.2 \\
6.0 \\
4.3 \\
1.3 \\
1.7 \\
6.6 \\
1.7 \\
5.0 \\
0.5\end{array}$ & $\begin{array}{l}3.7 \\
5.9 \\
4.6 \\
1.9 \\
1.3 \\
1.6 \\
1.7 \\
8.1 \\
1.2\end{array}$ & $\begin{array}{r}2.7 \\
5.6 \\
6.0 \\
1.4 \\
1.5 \\
0.8 \\
1.4 \\
12.3 \\
2.0\end{array}$ & $\begin{array}{l}2.8 \\
4.5 \\
4.2 \\
0.8 \\
1.8 \\
0.7 \\
1.6 \\
7.3 \\
2.9\end{array}$ & $\begin{array}{l}3.2 \\
5.2 \\
4.9 \\
0.7 \\
2.0 \\
1.1 \\
1.9 \\
9.0 \\
2.0\end{array}$ & $\begin{array}{l}2.0 \\
3.0 \\
3.9 \\
1.0 \\
2.1 \\
1.2 \\
2.0 \\
5.2 \\
3.9\end{array}$ & $\begin{array}{l}3.0 \\
2.9 \\
3.0 \\
1.4 \\
3.3 \\
1.0 \\
2.1 \\
4.4 \\
3.2\end{array}$ & $\begin{array}{l}3.9 \\
4.3 \\
3.2 \\
1.4 \\
5.2 \\
0.7 \\
2.2 \\
4.1 \\
2.6\end{array}$ & $\begin{array}{l}1 \\
? \\
2\end{array}$ & $\begin{array}{l}0.0 \\
0.0 \\
0.0 \\
0.0 \\
0.0 \\
0.0 \\
0.00 \\
0.0 \\
0.0\end{array}$ & 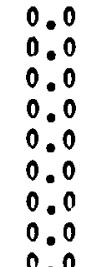 \\
\hline
\end{tabular}

30 Dearees North Latitude

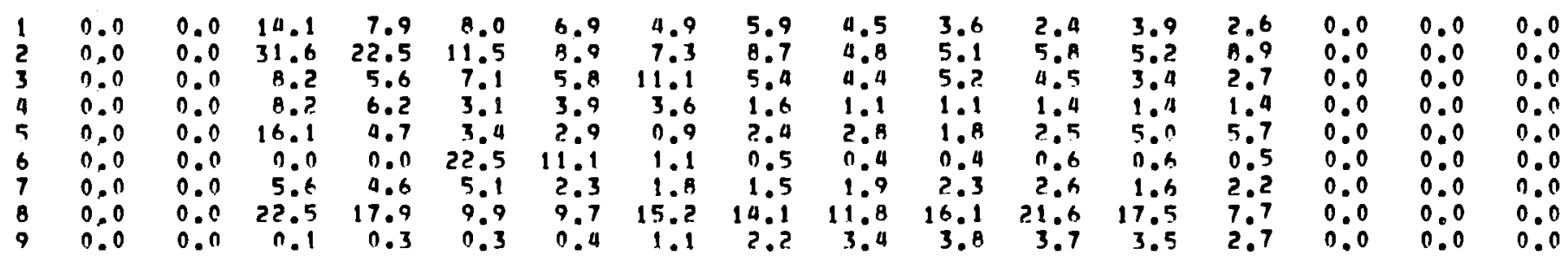




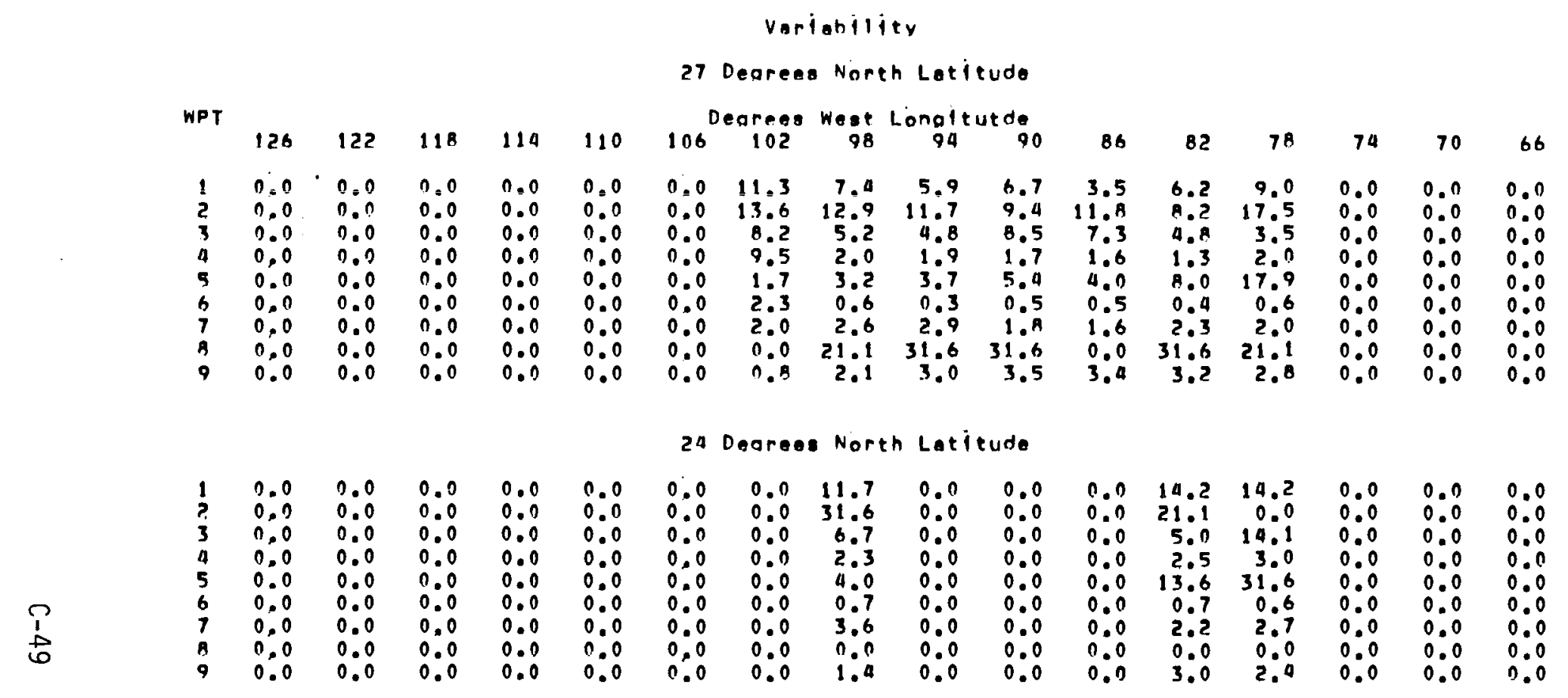


APPENDIX D 



\section{PERCENTAGE PRCBABILITY OF RUN DURATION EXCEEDANCE}

The data listings in this appendix present the percentage probability of a run of a weather pattern type exceeding the current duration. That is, if the run is already 2 days long (two consecutive days with the same weather pattern), the value given is the probability that the next day will also have the same weather pattern. Nine sets of tables are given, one for each current run weather pattern type. Summaries are presented for the six regions after these tables.

All of the tables are presented by latitude band across the region. The 10 bands are presented from north to south. Seventeen columns of data are presented in each band. The first column denotes the current run duration in days. The other 16 columns are headed by the longitude of the grid point for which the percentage probability of run duration exceedance applies.

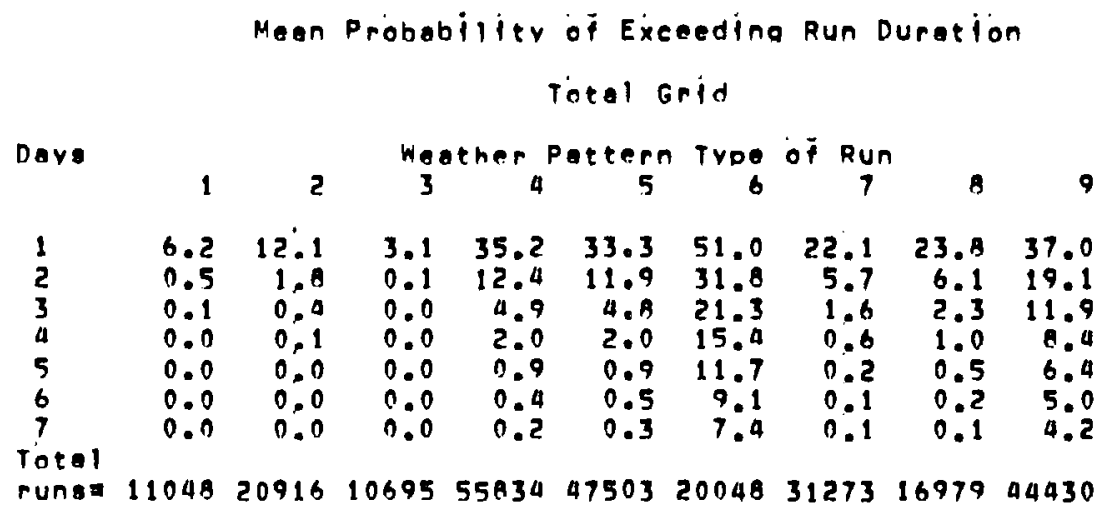


Probebility Run of WPT 1 will Exceed Duration Shown

51 Dearaes North Lotitude

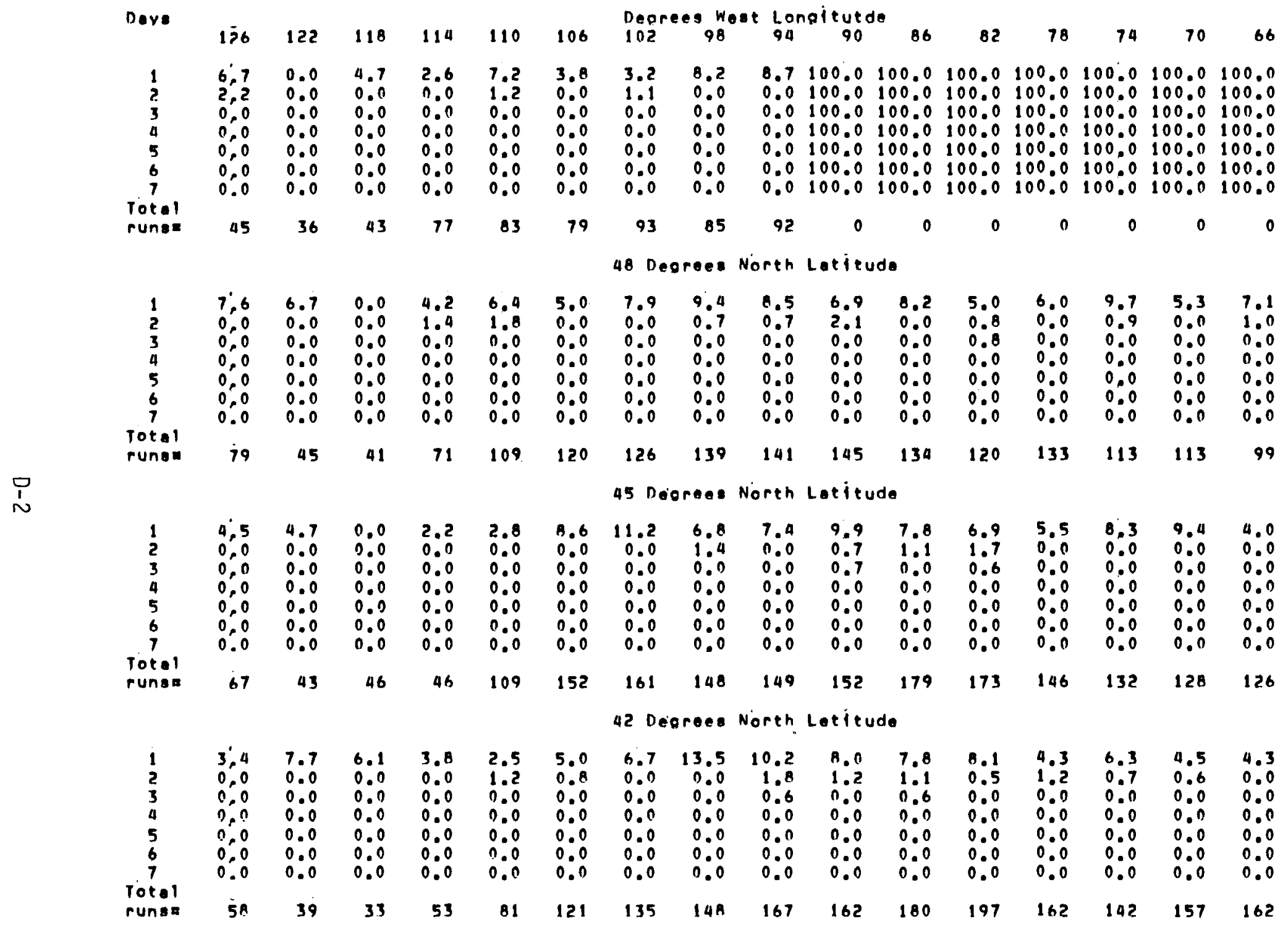


Probability Run of RPT 1 will Excead Duration shown

39 Degroes North Lotitude

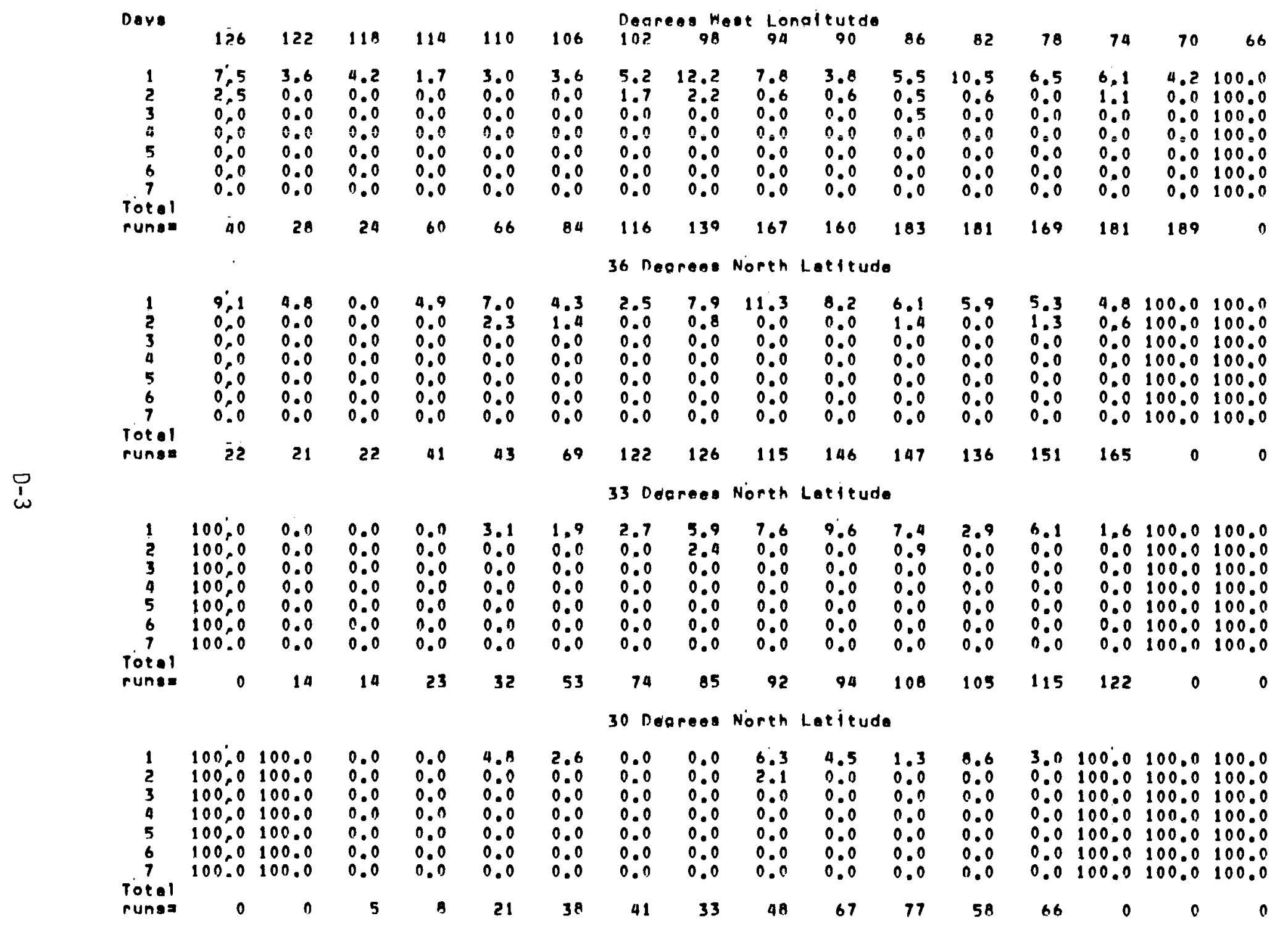




\section{Degreas North Lotitude}

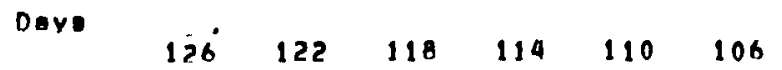

$100.0 \quad 100.0 \quad 100.0 \quad 100.0 \quad 100.0100 .0$ 100.0100 .0100 .0100 .0100 .0100 .0 $100,0100.0100 .0100 .0100 .0100 .0$ 100.0100 .0100 .0100 .0100 .0100 .0 100.0100 .0100 .0100 .0100 .0100 .0 1000100.0100 .0100 .0100 .0100 .0 100.0100 .0100 .0100 .0100 .0100 .0

\section{Degrees West Lonoltutde

$102^{0} 90^{0} 9 a^{0} 90 \quad 86$

$\begin{array}{lllll}0.0 & 0.0 & 2.9 & 4.7 & 0.0 \\ 0.0 & 0.0 & 0.0 & 0.0 & 0.0\end{array}$

0.0

$0.0 \quad 0.0 \quad 0.0 \quad 0.0 \quad 0.0$

$\begin{array}{lllll}0.0 & 0.0 & 0.0 & 0.0 & 0.0\end{array}$

$\begin{array}{lllll}0.0 & 0.0 & 0.0 & 0.0 & 0.0\end{array}$

0.0

0.0

24 Depreen North Latitude

$100.0100 .0 \quad 100.0100 .0100 .0100 .0100 .0$ $100.0 \quad 100.0 \quad 100.0 \quad 100.0 \quad 100.0 \quad 100.0100 .0$ 100.0100 .0100 .0100 .0100 .0100 .0100 .0 100.0100 .0100 .0100 .0100 .0100 .0100 .0 100.0100 .0100 .0100 .01100 .0100 .01100 .0 100.0100 .0100 .0100 .0100 .0100 .01100 .0 100.0100 .0100 .0100 .0100 .0100 .0100 .0

$0.0 \quad 100.01100 .0100 .0$ 0.0100 .0100 .0100 .0 0.0100 .0100 .0100 .0 0.0100 .0100 .0100 .0 0.0100 .0100 .0100 .0 0.0100 .0100 .0100 .0 0.0100 .0100 .0100 .0

0
0.0
0.0
0.0
0.0
0.0
2
7 74 70 66 $0.0 \quad 4.3 \quad 100.0 \quad 100.0100 .0$ 0.0100 .0100 .0100 .0 0.0100 .0100 .0100 .0 $0.0 \quad 0.0100 .0100 .0100 .0$ 0.0100 .0100 .0100 .0 $0.0 \quad 0.0100 .0100 .0100 .0$ $0.0100 .0 \quad 100.0100 .0$

$33.3 \quad 0.0 \quad 100.0 \quad 100.0 \quad 100.0$ $0.0 \quad 0.0 \quad 100.0 \quad 100.0 \quad 100.0$ $0.0 \quad 0.0 \quad 100.0 \quad 100.0100 .0$ $0.0 \quad 0.0100 .0100 .0100 .0$ $0.0 \quad 0.0100 .0100 .0100 .0$ $0.0 \quad 0.0100 .0100 .01100 .0$ $0.0 \quad 0.0100 .0100 .0100 .0$ Tote runse 
Probobility Run of hPt 2 will Excood Duration Shown

51 Degrees North Latitude

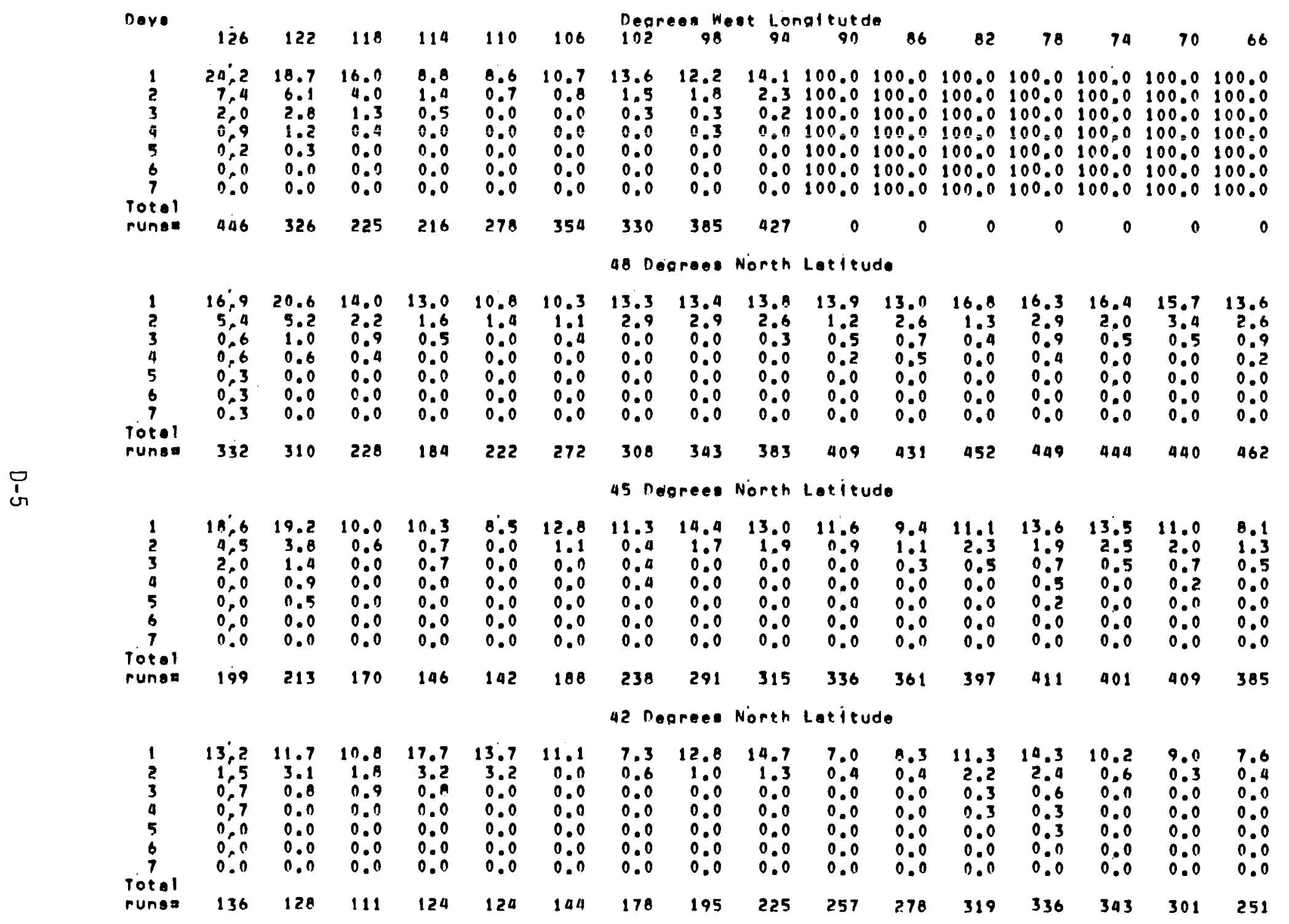


Probability Run of MPT 2 Will Excend Duretion shown

39 Deoraes North Latitude

\begin{tabular}{|c|c|c|c|c|c|c|c|c|c|c|c|c|c|c|c|c|}
\hline Deve & 126 & 122 & 118 & 114 & 110 & 106 & $\begin{array}{l}\text { Desr } \\
102\end{array}$ & $\begin{array}{c}\text { West } \\
98\end{array}$ & $\begin{array}{l}\text { L.on } \\
94\end{array}$ & $\begin{array}{l}\text { tutde } \\
\text { 9n }\end{array}$ & 86 & 82 & 78 & 74 & 10 & 66 \\
\hline $\begin{array}{l}1 \\
2 \\
3 \\
4 \\
5 \\
6 \\
7\end{array}$ & $\begin{array}{r}10: 3 \\
1,7 \\
1,7 \\
0,0 \\
0,0 \\
0,0 \\
0.0\end{array}$ & $\begin{array}{r}11.0 \\
0.0 \\
0.0 \\
0.0 \\
0.0 \\
0.0 \\
0.0\end{array}$ & $\begin{array}{r}12.2 \\
1.2 \\
0.0 \\
0.0 \\
0.0 \\
0.0 \\
0.0\end{array}$ & $\begin{array}{r}10.3 \\
1.0 \\
0.0 \\
0.0 \\
0.0 \\
0.0 \\
0.0\end{array}$ & $\begin{array}{r}13.2 \\
0.0 \\
0.0 \\
0.0 \\
0.0 \\
0.0 \\
0.0\end{array}$ & $\begin{array}{r}13.5 \\
1.0 \\
0.0 \\
0.0 \\
0.0 \\
0.0 \\
0.0\end{array}$ & $\begin{array}{l}8.1 \\
0.0 \\
0.0 \\
0.0 \\
0.0 \\
0.0 \\
0.0\end{array}$ & $\begin{array}{l}9.0 \\
0.0 \\
0.0 \\
0.0 \\
0.0 \\
0.0 \\
0.0\end{array}$ & $\begin{array}{l}0.5 \\
1.1 \\
0.0 \\
0.0 \\
0.0 \\
0.0 \\
0.0\end{array}$ & $\begin{array}{l}8.7 \\
0.0 \\
0.0 \\
0.0 \\
0.0 \\
0.0 \\
0.0\end{array}$ & $\begin{array}{l}5.1 \\
0.5 \\
0.0 \\
0.0 \\
0.0 \\
0.0 \\
0.0\end{array}$ & $\begin{array}{r}11.0 \\
0.8 \\
0.0 \\
0.0 \\
0.0 \\
0.0 \\
0.0\end{array}$ & $\begin{array}{r}13.6 \\
1.6 \\
0.4 \\
0.0 \\
0.0 \\
0.0 \\
0.0\end{array}$ & $\begin{array}{l}0.7 \\
0.5 \\
0.0 \\
0.0 \\
0.0 \\
0.0 \\
0.0\end{array}$ & $\begin{array}{l}4.7 \\
0.0 \\
0.0 \\
0.0 \\
0.0 \\
0.0 \\
0.0\end{array}$ & $\begin{array}{l}100.0 \\
100.0 \\
100.0 \\
100.0 \\
100.0 \\
100.0 \\
100.0\end{array}$ \\
\hline
\end{tabular}

36 Dearees North Latitude

\begin{tabular}{|c|c|c|c|c|c|c|c|c|c|c|c|c|c|c|c|c|}
\hline $\begin{array}{l}1 \\
2 \\
3 \\
9 \\
5 \\
6 \\
7\end{array}$ & $\begin{array}{r}11,1 \\
5,6 \\
0,0 \\
0,0 \\
0,0 \\
0,0 \\
0,0\end{array}$ & $\begin{array}{ll}4 & .2 \\
0 & 0 \\
0 & 0 \\
0 & 0 \\
0 & 0 \\
0 & 0 \\
0.0 & 0 \\
0.0 & 0 \\
0.0 & 0\end{array}$ & $\begin{array}{l}6.1 \\
0.0 \\
0.0 \\
0.0 \\
0.0 \\
0.0 \\
0.0\end{array}$ & $\begin{array}{l}2.4 \\
0.0 \\
0.0 \\
0.0 \\
0.0 \\
0.0 \\
0.0\end{array}$ & $\begin{array}{l}7.7 \\
0.0 \\
0.0 \\
0.0 \\
0.0 \\
0.0 \\
0.0\end{array}$ & $\begin{array}{l}9.3 \\
1.9 \\
0.0 \\
0.0 \\
0.0 \\
0.0 \\
0.0\end{array}$ & $\begin{array}{l}6.3 \\
0.0 \\
0.0 \\
0.0 \\
0.0 \\
0.0 \\
0.0\end{array}$ & $\begin{array}{l}6.7 \\
0.0 \\
0.0 \\
0.0 \\
0.0 \\
0.0 \\
0.0\end{array}$ & $\begin{array}{r}11.0 \\
0.0 \\
0.0 \\
0.0 \\
0.0 \\
0.0 \\
0.0\end{array}$ & $\begin{array}{l}5.2 \\
0.0 \\
0.0 \\
0.0 \\
0.0 \\
0.0 \\
0.0\end{array}$ & $\begin{array}{l}6.6 \\
0.0 \\
0.0 \\
0.0 \\
0.0 \\
0.0 \\
0.0\end{array}$ & $\begin{array}{l}9.3 \\
1.3 \\
0.0 \\
0.0 \\
0.0 \\
0.0 \\
0.0\end{array}$ & $\begin{array}{l}7.0 \\
0.0 \\
0.0 \\
0.0 \\
0.0 \\
0.0 \\
0.0\end{array}$ & $\begin{array}{l}1.3 \\
0.0 \\
0.0 \\
0.0 \\
0.0 \\
0.0 \\
0.0 \\
0.0\end{array}$ & $\begin{array}{l}100.0 \\
100.0 \\
100.0 \\
100.0 \\
100.0 \\
100.0 \\
100.0\end{array}$ & $\begin{array}{l}100.0 \\
100.0 \\
100.0 \\
100.0 \\
100.0 \\
100.0 \\
100.0\end{array}$ \\
\hline $\begin{array}{l}\text { Dtal } \\
\text { ungar }\end{array}$ & 18 & 24 & 33 & 41 & 39 & 54 & 79 & 89 & 110 & 116 & 152 & 151 & 128 & 92 & 0 & 0 \\
\hline
\end{tabular}

$\stackrel{p}{a}$

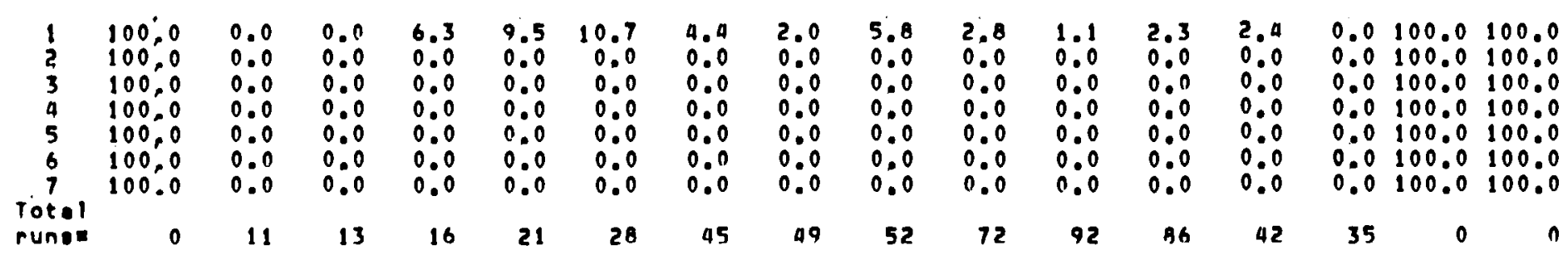

30 negrees North Latitude

$\begin{array}{rrrrrrrrrrrrrrrrrr}1 & 100.0 & 100.0 & 0.0 & 50.0 & 11.1 & 18.2 & 0.3 & 0.0 & 5.6 & 5.3 & 0.0 & 0.0 & 0.0 & 100.0 & 100.0 & 100.0 \\ 2 & 100.0 & 100.0 & 0.0 & 0.0 & 0.0 & 0.0 & 0.0 & 0.0 & 0.0 & 0.0 & 0.0 & 0.0 & 0.0 & 100.0 & 100.0 & 100.0 \\ 3 & 100.0 & 100.0 & 0.0 & 0.0 & 0.0 & 0.0 & 0.0 & 0.0 & 0.0 & 0.0 & 0.0 & 0.0 & 0.0 & 100.0 & 100.0 & 100.0 \\ 0 & 100.0 & 100.0 & 0.0 & 0.0 & 0.0 & 0.0 & 0.0 & 0.0 & 0.0 & 0.0 & 0.0 & 0.0 & 0.0 & 100.0 & 100.0 & 100.0 \\ 5 & 100.0 & 100.0 & 0.0 & 0.0 & 0.0 & 0.0 & 0.0 & 0.0 & 0.0 & 0.0 & 0.0 & 0.0 & 0.0 & 100.0 & 100.0 & 100.0 \\ 6 & 100.0 & 100.0 & 0.0 & 0.0 & 0.0 & 0.0 & 0.0 & 0.0 & 0.0 & 0.0 & 0.0 & 0.0 & 0.0 & 100.0 & 100.0 & 100.0 \\ 7 & 100.0 & 100.0 & 0.0 & 0.0 & 0.0 & 0.0 & 0.0 & 0.0 & 0.0 & 0.0 & 0.0 & 0.0 & 0.0 & 100.0 & 100.0 & 100.0 \\ \text { rotel } & 1000 & & 0 & 1 & 2 & 9 & 11 & 24 & 25 & 36 & 38 & 42 & 33 & 13 & 0 & 0 & 0\end{array}$


Probohility Run of hPT 2 Will Exceed Duration shown

\section{Degrane North Latitude}

Devo
136
122
118
114
110
106

100.0100 .0100 .0100 .0100 .0100 .0 100.0100 .0100 .0100 .0100 .0100 .0 100.0100 .0100 .0100 .0100 .0100 .0 $100,0100.0 \quad 100.0100 .0100 .0100 .0$ $100.0100 .0 \quad 100.0100 .0100 .0100 .0$ 100.0100 .0100 .0100 .01100 .01100 100.0100 .0100 .0100 .0100 .0100 .0

Totel

runes

\section{Deareses West Longitut de}

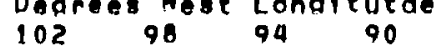

0.0

0.0

0.0

0.0

$0.0 \quad 0.0$

$0.0 \quad 0.0$

$0.0 \quad 0.0$

$0.0 \quad 0.0$

0.0
0.0

0.0

0.0

0.0

0.0

86

82
0.0
0.0
0.0
0.0
0.0
0.0
0.0
10

78

74

70

66

0.0100 .0100 .0100 .0 $0.0100 .0 \quad 100.0 \quad 100.0$ 0.0100 .0100 .0100 .0 0.0100 .0100 .0100 .0 0.0100 .0100 .0100 .0 0.0100 .0100 .0100 .0 0.0100 .0100 .0100 .0 $0.0 \quad 0.0 \quad 0.0$

24 Deorece North Letitude

$1 \quad 100.0100 .0100 .0100 .0100 .0100 .0100 .0$ $100.0 \quad 100.0 \quad 100.0 \quad 100.0 \quad 100.0 \quad 100.0 \quad 100.0$ $100,0 \quad 100.0 \quad 100.0 \quad 100.0 \quad 100.0 \quad 100.0 \quad 100.0$ $100,0100.0 \quad 100.0 \quad 100.0 \quad 100.0 \quad 100.0 \quad 100.0$ $100.0 \quad 100.0100 .0 \quad 100.0100 .0100 .0100 .0$ $100.0 \quad 100.0100 .0100 .0100 .0100 .0100 .0$ 7 100.0100 .0100 .0100 .0100 .0100 .0100 .0

0.0100 .0100 .0100 .0 0.0100 .0100 .0100 .0 0.0100 .0100 .0100 .0 0.0100 .0100 .0100 .0 0.0100 .0100 .0100 .0 0.0100 .0100 .0100 .0

$\begin{array}{llll}0.0 & 100.0 & 100.0 & 100.0 \\ 0.0 & 100.0 & 100.0 & 100.0\end{array}$

0.0100 .0100 .0100 .0100 .0 $0.0 \quad 100.0 \quad 100.0 \quad 100.0 \quad 100.0$ 0.0100 .0100 .0100 .0100 .0 $0.0100 .0 \quad 100.0 \quad 100.0 \quad 100.0$ 0.0100 .0100 .0100 .0100 .0 0.0100 .0100 .0100 .0100 .0

0.0100 .0100 .0100 .0100 .0 Total

0

0

0

1

0

0

. 2

0

0

0 0 
51 Deorees North Latitude

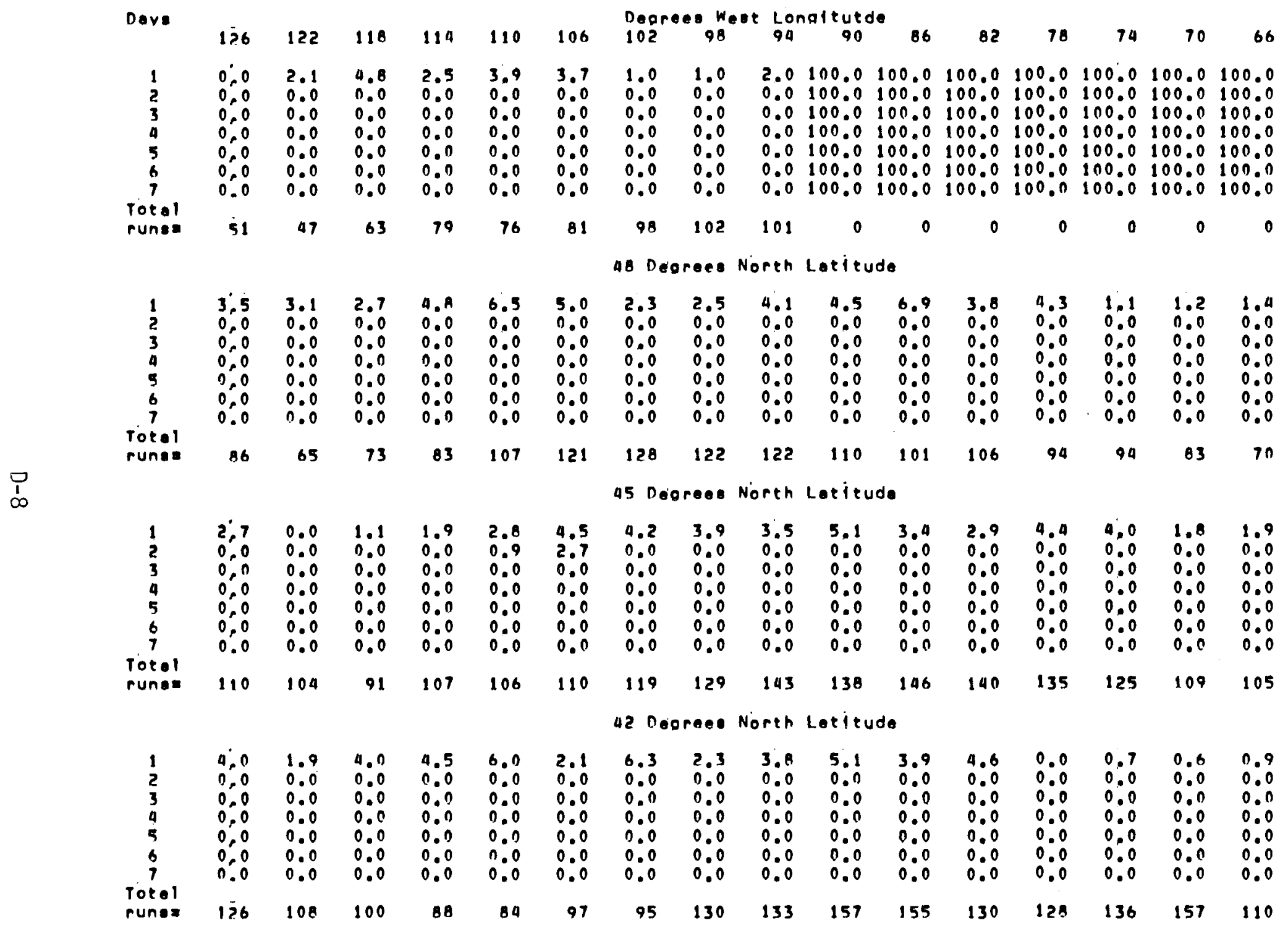


Probehility Run of MPT 3 will Exceed Duration shown

39 Degrees North Latitude

\begin{tabular}{|c|c|c|c|c|c|c|c|c|c|c|c|c|c|c|c|c|}
\hline Dere & 126 & 122 & 118 & 114 & 110 & 106 & $\begin{array}{l}\text { Deg } \\
102\end{array}$ & $\underbrace{W_{0}}_{9 B}$ & $\begin{array}{l}\text { Lor } \\
94\end{array}$ & $\begin{array}{c}\text { tut de } \\
90\end{array}$ & $B 6$ & B2 & 78 & 74 & 70 & 66 \\
\hline $\begin{array}{c}1 \\
2 \\
3 \\
4 \\
5 \\
6 \\
7 \\
\text { Totol }\end{array}$ & $\begin{array}{ll}3 & 0 \\
0 & 0 \\
0 & 0 \\
0 & 0 \\
0 & 0 \\
0 & 0 \\
0 & 0\end{array}$ & $\begin{array}{l}1.0 \\
0.0 \\
0.0 \\
0.0 \\
0.0 \\
0.0 \\
0.0\end{array}$ & $\begin{array}{l}5.2 \\
0.0 \\
0.0 \\
0.00 \\
0.0 \\
0.0 \\
0.0\end{array}$ & $\begin{array}{l}4.1 \\
0.0 \\
0.0 \\
0.0 \\
0.0 \\
0.0 \\
0.0\end{array}$ & $\begin{array}{l}1.0 \\
0.0 \\
0.0 \\
0.0 \\
0.0 \\
0.0 \\
0.0\end{array}$ & $\begin{array}{ll}4 & 2 \\
0.0 & 0 \\
0.0 & 0 \\
0 & 0 \\
0.0 & 0 \\
0.0 & 0 \\
0.0 & 0\end{array}$ & $\begin{array}{l}7.0 \\
0.0 \\
0.0 \\
0.0 \\
0.0 \\
0.0 \\
0.0\end{array}$ & $\begin{array}{l}2.7 \\
0.9 \\
0.0 \\
0.0 \\
0.0 \\
0.0 \\
0.0\end{array}$ & $\begin{array}{l}3.2 \\
0.0 \\
0.0 \\
0.0 \\
0.0 \\
0.00 \\
0.0\end{array}$ & $\begin{array}{l}3.7 \\
0.0 \\
0.0 \\
0.0 \\
0.0 \\
0.0 \\
0.0\end{array}$ & $\begin{array}{l}2.0 \\
0.00 \\
0.00 \\
0.00 \\
0.00 \\
0.00 \\
0.00\end{array}$ & $\begin{array}{l}2.2 \\
0.0 \\
0.00 \\
0.00 \\
0.00 \\
0.00 \\
0.0\end{array}$ & $\begin{array}{l}2.0 \\
0.0 \\
0.0 \\
0.0 \\
0.0 \\
0.0 \\
0.0\end{array}$ & $\begin{array}{l}2.7 \\
0.0 \\
0.0 \\
0.0 \\
0.0 \\
0.0 \\
0.0\end{array}$ & $\begin{array}{l}0.0 \\
0.0 \\
0.0 \\
0.0 \\
0.0 \\
0.0 \\
0.0\end{array}$ & $\begin{array}{l}100.0 \\
100.0 \\
100.0 \\
100.0 \\
100.0 \\
100.0 \\
100.0\end{array}$ \\
\hline
\end{tabular}

36 Degrees North Latitude

\begin{tabular}{|c|c|c|c|c|c|c|c|c|c|c|c|c|c|c|c|c|}
\hline $\begin{array}{l}1 \\
2 \\
3 \\
4 \\
5 \\
6 \\
7 \\
\text { rotan } 1\end{array}$ & $\begin{array}{l}3: 4 \\
0 ; 0 \\
0=0 \\
0,0 \\
0=0 \\
0=0 \\
0=0 \\
5 B\end{array}$ & $\begin{array}{l}9.4 \\
1.9 \\
0.0 \\
0.0 \\
0.0 \\
0.0 \\
0.0 \\
53\end{array}$ & $\begin{array}{l}0.3 \\
0.0 \\
0.0 \\
0.0 \\
0.0 \\
0.0 \\
0.0 \\
79\end{array}$ & $\begin{array}{l}3.0 \\
0.0 \\
0.0 \\
0.0 \\
0.0 \\
0.0 \\
0.0 \\
66\end{array}$ & $\begin{array}{l}4.9 \\
0.0 \\
0.0 \\
0.0 \\
0.0 \\
0.0 \\
0.0 \\
41\end{array}$ & $\begin{array}{l}0.2 \\
0.0 \\
0.0 \\
0.0 \\
0.0 \\
0.0 \\
0.0 \\
71\end{array}$ & $\begin{array}{l}0.6 \\
0.0 \\
0.0 \\
0.0 \\
0.0 \\
0.0 \\
0.0 \\
07\end{array}$ & $\begin{array}{l}2.6 \\
0.0 \\
0.0 \\
0.0 \\
0.0 \\
0.0 \\
0.0 \\
76\end{array}$ & $\begin{array}{l}3.6 \\
0.0 \\
0.0 \\
0.0 \\
0.0 \\
0.0 \\
0.0 \\
110\end{array}$ & $\begin{array}{l}2.9 \\
0.0 \\
0.0 \\
0.0 \\
0.0 \\
0.0 \\
0.0 \\
105\end{array}$ & $\begin{array}{l}2.5 \\
0.8 \\
0.0 \\
0.0 \\
0.0 \\
0.0 \\
0.0 \\
121\end{array}$ & $\begin{array}{l}4.6 \\
0.0 \\
0.0 \\
0.0 \\
0.0 \\
0.0 \\
0.0 \\
130\end{array}$ & $\begin{array}{l}3.0 \\
0.0 \\
0.0 \\
0.0 \\
0.0 \\
0.0 \\
0.0 \\
134\end{array}$ & $\begin{array}{l}4.1 \\
0.0 \\
0.0 \\
0.0 \\
0.0 \\
0.0 \\
0.0 \\
146\end{array}$ & $\begin{array}{r}100.0 \\
100.0 \\
100.0 \\
100.0 \\
100.0 \\
100.0 \\
100.0 \\
0\end{array}$ & $\begin{array}{l}100.0 \\
100.0 \\
100.0 \\
100.0 \\
100.0 \\
100.0 \\
100.0\end{array}$ \\
\hline
\end{tabular}

\section{Desrees North Latieude}

$\begin{array}{rrrrrrrrrrr}1.3 & 1.7 & 1.3 & 0.0 & 3.3 & 5.8 & 1.9 & 1.9 & 100.0 & 100.0 \\ 0.0 & 0.0 & 0.0 & 0.0 & 0.0 & 1.0 & 0.0 & 0.0 & 1000.0 & 100.0 \\ 0.0 & 0.0 & 0.0 & 0.0 & 0.0 & 0.0 & 0.0 & 0.0 & 100.0 & 100.0 \\ 0.0 & 0.0 & 0.0 & 0.0 & 0.0 & 0.0 & 0.0 & 0.0 & 100.0 & 100.0 \\ 0.0 & 0.0 & 0.0 & 0.0 & 0.0 & 0.0 & 0.0 & 0.0 & 100.0 & 100.0 \\ 0.0 & 0.0 & 0.0 & 0.0 & 0.0 & 0.0 & 0.0 & 0.0 & 100.0 & 100.0 \\ 0.0 & 0.0 & 0.0 & 0.0 & 0.0 & 0.0 & 0.0 & 0.0 & 100.0 & 100.0 \\ 75 & 59 & 78 & 73 & 90 & 103 & 105 & 104 & 0 & 0\end{array}$

30 Deoreas North Letitude

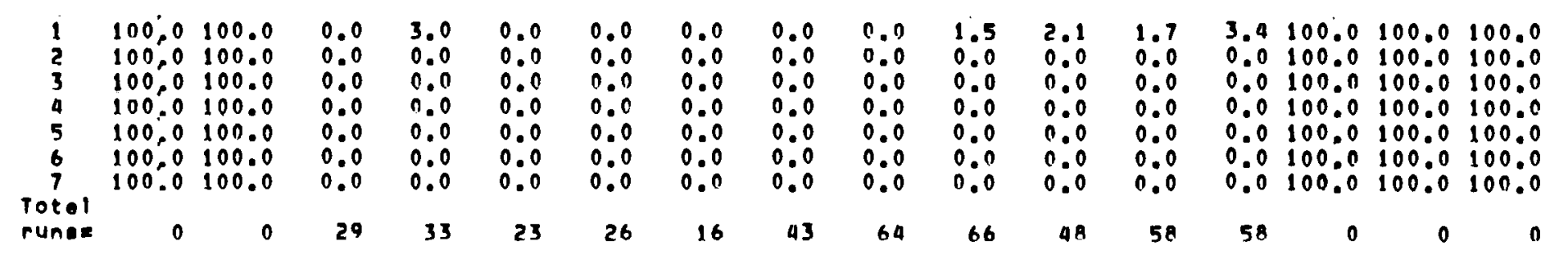


27 Dearees North Latitude

Daye

$\begin{array}{llllll}126 & 122 & 118 & 114 & 110 & 106\end{array}$
Degrees Wost Longltutde
102

\section{$0.0 \quad 0.0$}

$100,0100.0100 .0100 .0100 .0$ 100.0100 .0100 .0100 .01100 .0100 .0 100.0100 .0100 .0101 .0100 .0100 .0 100.0100 .0100 .0100 .0100 .0100 .0 100.0100 .01100 .0100 .0100 .0100 .0 100.0100 .0100 .0101 .01100 .0100 .0 100.0100 .0100 .0100 .0100 .0100 .0 Totel 1 runea

0
0
0
0

0.0
0.0
0.0
0.0
0.0
0.0
0.0
21

$\begin{array}{ll}0.0 & 3.3 \\ 0.0 & 0.0 \\ 0.0 & 0.0 \\ 0.0 & 0.0 \\ 0.0 & 0.0 \\ 0.0 & 0.0 \\ 0.0 & 0.0 \\ & \\ 44 & 30\end{array}$

24 Dogroes North Latitude

$100.0100 .0 \quad 100.0 \quad 100.0 \quad 100.0100 .0 \quad 100.0$ $100.0 \quad 100.0 \quad 100.0 \quad 100.0 \quad 100.0 \quad 100.0 \quad 100.0$ $100.0100 .0100 .0100 .0 \quad 100.0100 .0100 .0$ $100,0100.0100 .0100 .0100 .0100 .0100 .0$ $100.0100 .0100 .0 \quad 100.0100 .0100 .0100 .0$ $100.0100 .0 \quad 100.0 \quad 100.0100 .0 \quad 100.0100 .0$ 100.0100 .0100 .0100 .0100 .0100 .0100 .0

0.0100 .0100 .0100 .0 $0.0100 .0 \quad 100.0 \quad 100.0$ $0.0100 .0100 .0 \quad 100.0$ 0.0100 .0100 .0100 .0 0.0100 .0100 .0100 .0 0.0100 .0100 .0100 .0 0.0100 .0100 .0100 .0 82 0.0 0.0 0.0 0.0 0.0

\section{2}

0.0

$$
0.0
$$
0.0

78 74

70 66

.0100 .0100 .0100 .0 $0.0100 .0100 .0 \quad 100.0$ $0.0 \quad 100.0 \quad 100.0 \quad 100.0$ $.0100 .0 \quad 100.0 \quad 100.0$ 0.0100 .0100 .0100 .0 0.0100 .0100 .0100 .0 0.0100 .0100 .0100 .0

31

32

$$
0
$$

$0.0 \quad 0.0 \quad 100.0 \quad 100.0 \quad 100.0$ $0.0 \quad 0.0 \quad 100.0 \quad 100.0100 .0$ $0.0 \quad 0.0 \quad 100.0 \quad 100.0 \quad 100.0$ $0.0 \quad 0.0 \quad 100.0 \quad 100.0100 .0$ $\begin{array}{llllll}0.0 & 0.0 & 100.0 & 100.0 & 100.0 \\ 0.0 & 0.0 & 100.0 & 100.0 & 100.0\end{array}$ $\begin{array}{llllll}0.0 & 0.0 & 100.0 & 100.0 & 100.0 \\ 0.0 & 0.0 & 100.0 & 100.0 & 100.0\end{array}$ $0.0 \quad 0.0 \quad 100.0100 .0100 .0$ Totel

0

0

0

11

0

0

14

6

$0 \quad 0$ 
Probability Run of KPT 4 Will Exceed Duration shown

51 Peorees North Latitude

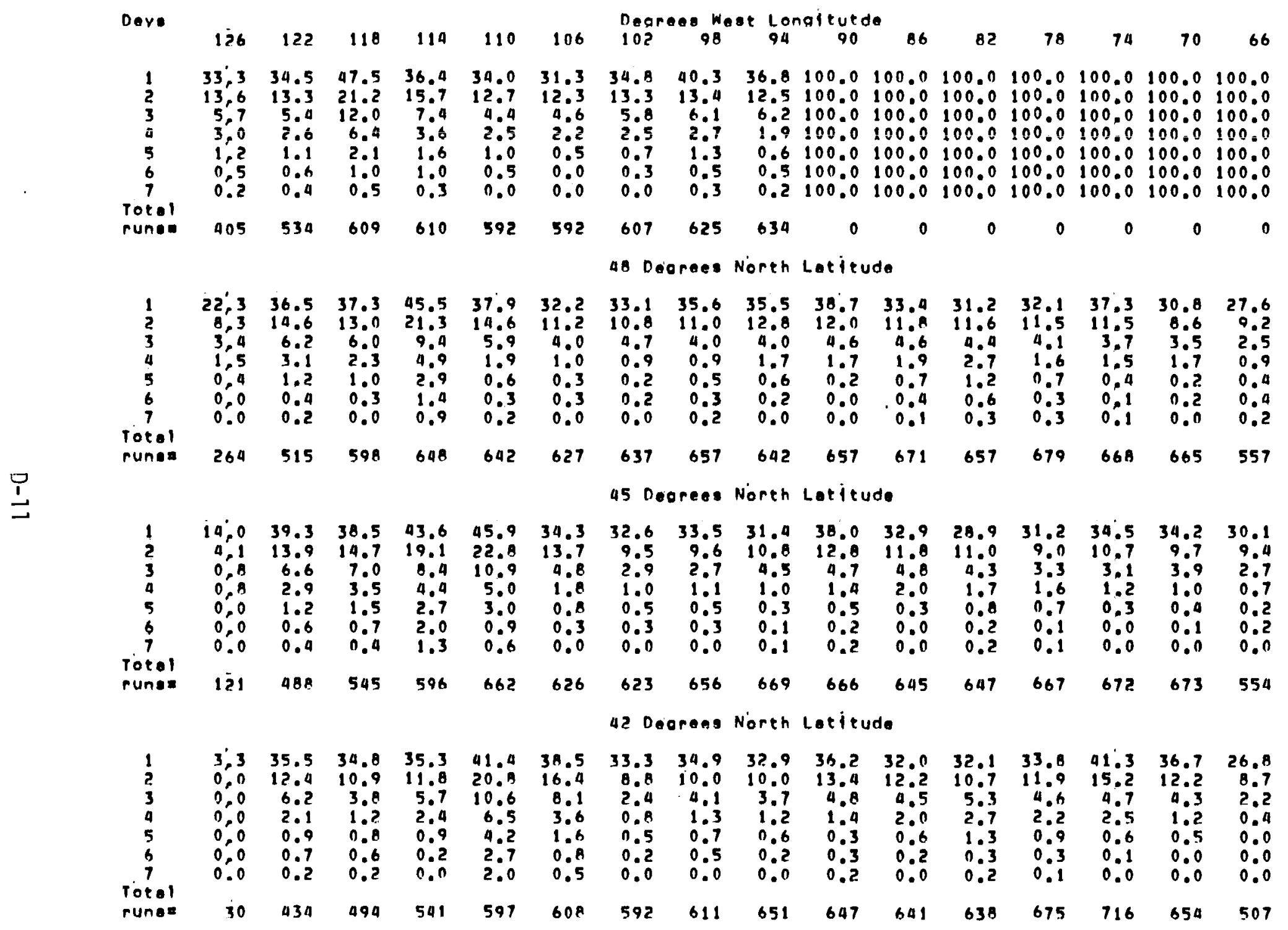


39 Degrees North Latitude

\begin{tabular}{|c|c|c|c|c|c|c|}
\hline Deve & 126 & 122 & 118 & 114 & 110 & 106 \\
\hline $\begin{array}{c}1 \\
2 \\
3 \\
4 \\
5 \\
6 \\
7 \\
\text { Total } \\
\text { runge }\end{array}$ & $\begin{array}{l}0,0 \\
0,0 \\
0,0 \\
0,0 \\
0 ; 0 \\
0,0 \\
0.0\end{array}$ & $\begin{array}{r}29.8 \\
9.7 \\
3.9 \\
1.6 \\
0.8 \\
0.0 \\
0.0 \\
258\end{array}$ & $\begin{array}{r}30.9 \\
9.8 \\
3.7 \\
1.3 \\
0.5 \\
0.0 \\
0.0 \\
376\end{array}$ & $\begin{array}{r}29.0 \\
9.5 \\
2.0 \\
0.7 \\
0.5 \\
0.0 \\
0.0 \\
011\end{array}$ & $\begin{array}{r}35.0 \\
13.4 \\
5.1 \\
3.1 \\
1.0 \\
1.4 \\
0.0 \\
509\end{array}$ & $\begin{array}{r}45.1 \\
25.2 \\
14.2 \\
7.4 \\
4.3 \\
3.1 \\
1.6 \\
556\end{array}$ \\
\hline
\end{tabular}

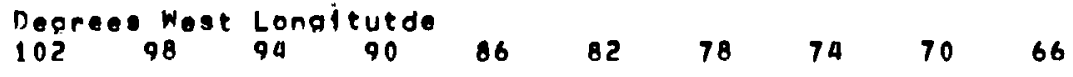

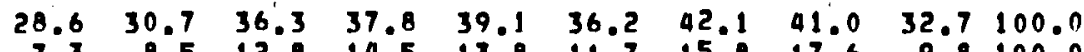

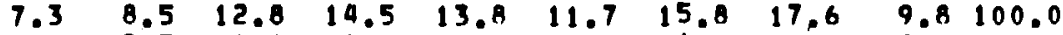

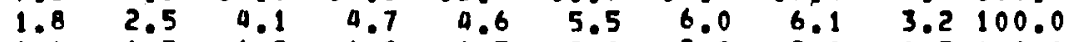

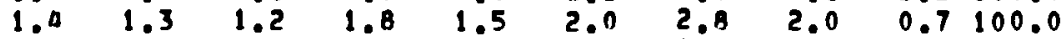

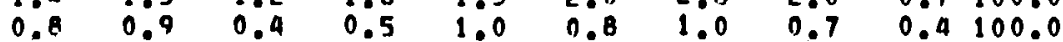

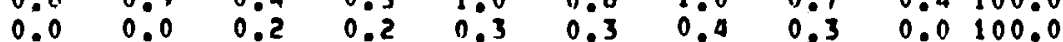

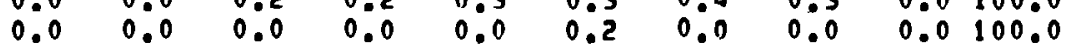

$\begin{array}{llllllllll}507 & 554 & 564 & 600 & 614 & 658 & 682 & 693 & 560 & 0\end{array}$

36 Degrees Noreh Latitude

$\begin{array}{crrrrrr}1 & 0.0 & 12.7 & 29.8 & 27.0 & 25.6 & 43.2 \\ 2 & 0.0 & 2.8 & 10.5 & 6.6 & 8.7 & 19.8 \\ 3 & 0.0 & 0.0 & 4.8 & 2.5 & 3.2 & 10.9 \\ 4 & 0.0 & 0.0 & 1.3 & 0.8 & 1.0 & 5.0 \\ 5 & 0.0 & 0.0 & 0.9 & 0.4 & 0.7 & 3.1 \\ 6 & 0.0 & 0.0 & 0.0 & 0.0 & 0.5 & 2.3 \\ 7 & 0.0 & 0.0 & 0.0 & 0.0 & 0.2 & 2.3 \\ \text { rotel } & & & & & & \\ \text { runge } & 3 & 71 & 228 & 241 & 0.03 & 516\end{array}$

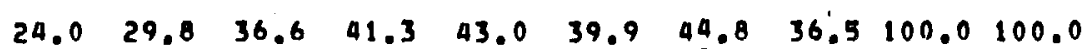

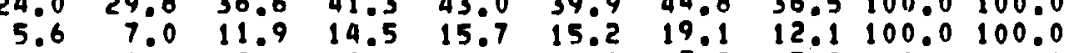

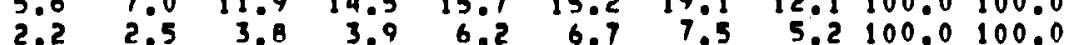

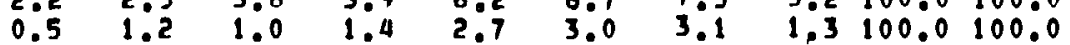

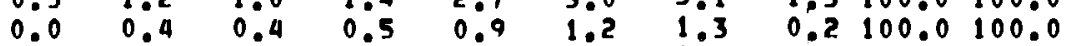

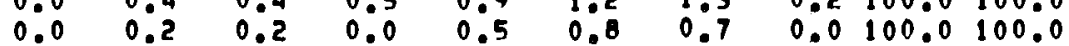

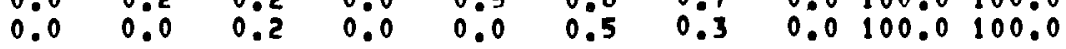

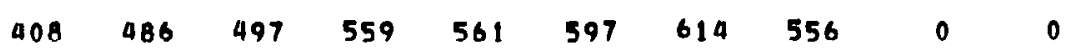

33 Déprees North Latitude

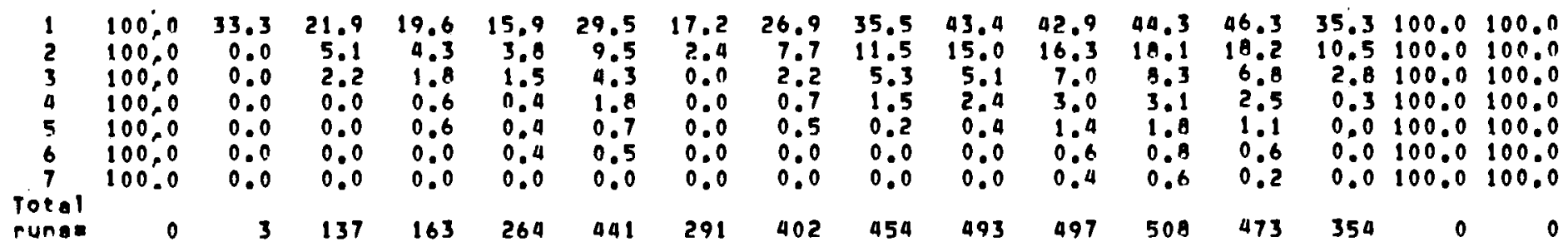

30 Degrees North Latitude

\begin{tabular}{|c|c|c|c|c|c|c|c|c|c|c|c|c|c|c|c|c|}
\hline $\begin{array}{l}1 \\
2 \\
3 \\
4 \\
5 \\
6 \\
7\end{array}$ & $\begin{array}{l}100,0 \\
100,0 \\
100,0 \\
100,0 \\
100,0 \\
100,0 \\
100.0\end{array}$ & $\begin{array}{l}100.0 \\
1000.0 \\
100.0 \\
100.0 \\
100.0 \\
100.0 \\
100.0\end{array}$ & $\begin{array}{l}6.3 \\
6.3 \\
0.0 \\
0.0 \\
0.0 \\
0.0 \\
0.0\end{array}$ & $\begin{array}{r}19.5 \\
3.7 \\
2.4 \\
0.0 \\
0.0 \\
0.0 \\
0.0\end{array}$ & $\begin{array}{r}12.8 \\
1.1 \\
1.1 \\
0.0 \\
0.0 \\
0.0 \\
0.0\end{array}$ & $\begin{array}{l}10.3 \\
1.5 \\
0.0 \\
0.0 \\
0.0 \\
0.0 \\
0.0\end{array}$ & $\begin{array}{l}5.5 \\
0.0 \\
0.0 \\
0.0 \\
0.0 \\
0.0 \\
0.0\end{array}$ & $\begin{array}{r}26.1 \\
6.4 \\
1.7 \\
0.9 \\
0.3 \\
0.0 \\
0.0\end{array}$ & $\begin{array}{r}40.1 \\
11.6 \\
4.2 \\
1.5 \\
0.7 \\
0.0 \\
0.0\end{array}$ & $\begin{array}{r}40.8 \\
13.5 \\
5.6 \\
1.5 \\
1.3 \\
0.8 \\
0.0\end{array}$ & $\begin{array}{r}41.6 \\
16.0 \\
8.0 \\
3.1 \\
1.8 \\
1.3 \\
0.5\end{array}$ & $\begin{array}{r}47.9 \\
16.1 \\
5.6 \\
2.6 \\
1.1 \\
0.5 \\
0.0\end{array}$ & $\begin{array}{r}41.0 \\
12.9 \\
4.7 \\
0.6 \\
0.0 \\
0.0 \\
0.0\end{array}$ & $\begin{array}{l}100.0 \\
100.0 \\
100.0 \\
100.0 \\
100.0 \\
100.0 \\
1000.0\end{array}$ & $\begin{array}{l}100.0 \\
100.0 \\
100.0 \\
100.0 \\
100.0 \\
100.0 \\
100.0\end{array}$ & $\begin{array}{l}100.0 \\
100.0 \\
100.0 \\
100.0 \\
100.0 \\
1000.0 \\
100.0\end{array}$ \\
\hline & 皮 & 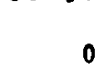 & 16 & 82 & $9 a$ & 136 & $12 \mathrm{~B}$ & 345 & 404 & 392 & 387 & 380 & 317 & 0 & 0 & 0 \\
\hline
\end{tabular}


Probobiliter Run of WPT 4 Will Exceed Duration shown

27 Dearoes North Latitude

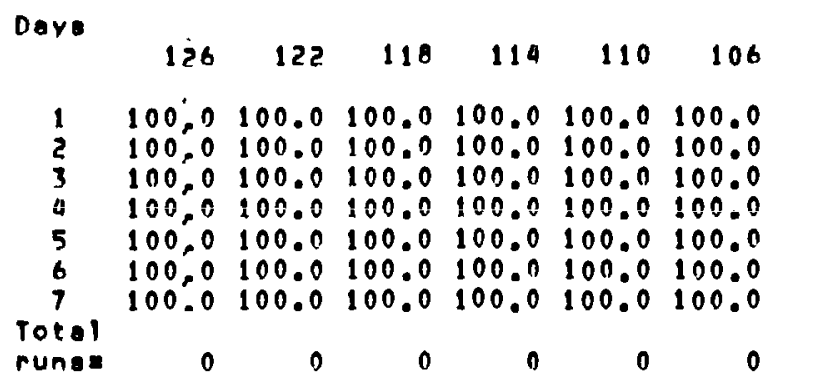

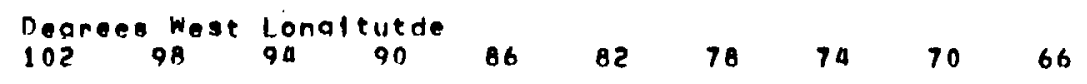

$2.9 \quad 29.9 \quad 35.9 \quad 34.2 \quad 36.6 \quad 37.0 \quad 31.4 \quad 100.0 \quad 100.0100 .0$

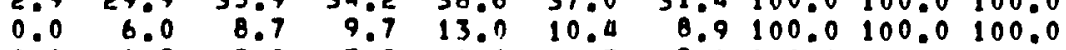

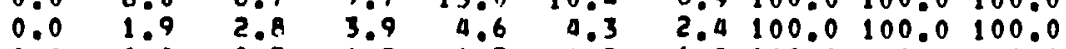

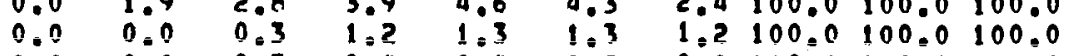

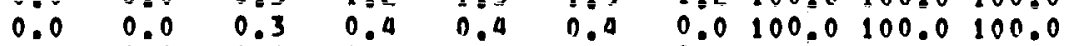

$0.0 \quad 0.0 \quad 0.3 \quad 0.4 \quad 0.0 \quad 0.0 \quad 0.0100 .0100 .0100 .0$ $0.0 \quad 0.0 \quad 0.0 \quad 0.0 \quad 0.0 \quad 0.0 \quad 0.0100 .0100 .0100 .0$

runas

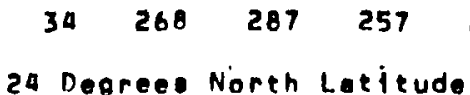

$1 \quad 100.0100 .0100 .0100 .0100 .0100 .0100 .0 \quad 25.4100 .0100 .0100 .0$ $100.0100 .0100 .0100 .0 \quad 100.0 \quad 100.0 \quad 100.0 \quad 3.6 \quad 100.0 \quad 100.0100 .0$ $100.0100 .0 \quad 100.0 \quad 100.0 \quad 100.0 \quad 100.0 \quad 100.0 \quad 1.0 \quad 100.0 \quad 100.0 \quad 100.0$ $100.0100 .0 \quad 100.0100 .0 \quad 100.0100 .0100 .0 \quad 0.0 \quad 100.0100 .0100 .0$ $100.0100 .0100 .0100 .0100 .0100 .0100 .0 \quad 0.0100 .0100 .0100 .0$

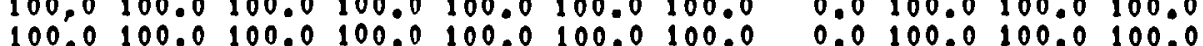

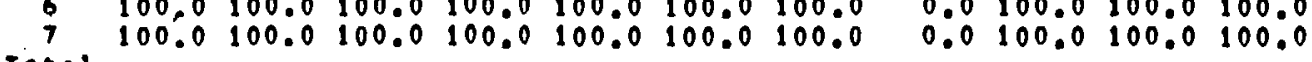

28.318 .3100 .0100 .0100 .0 a.0 1.4100 .0100 .0100 .0 $1.0 \quad 0.01100 .0100 .0100 .0$ $0.0 \quad 0.0100 .0100 .0100 .0$ 0.000 .0100 .0100 .0100 .0 $0.0 \quad 0.0100 .0100 .0100 .0$

runes
(20.90

(1)

0

0

193

(0

99

71 
51 Dearees North Latitude

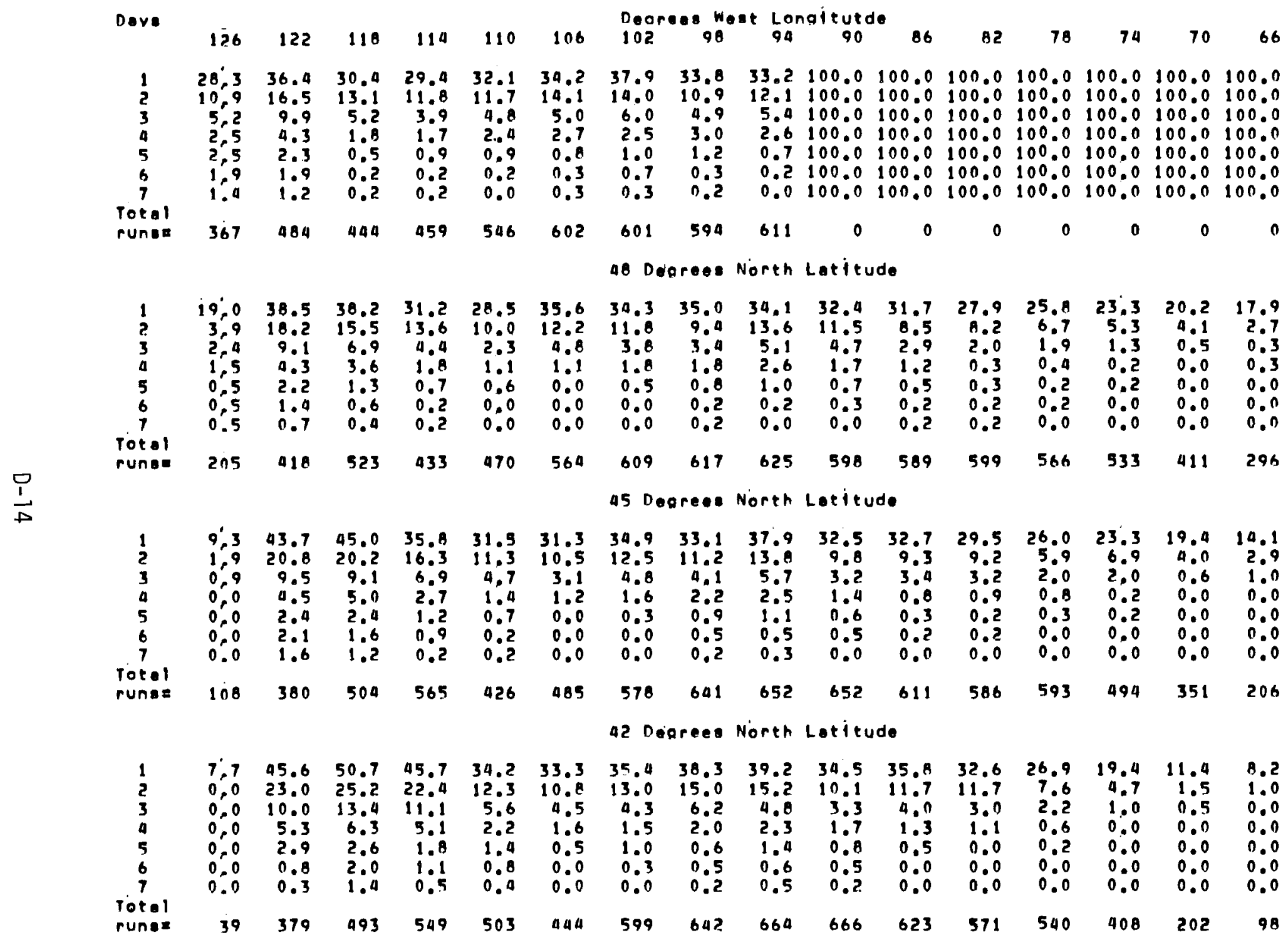


Probability Run of RPT 5 will Excend Duration shown

39 Dooreas North Latitude

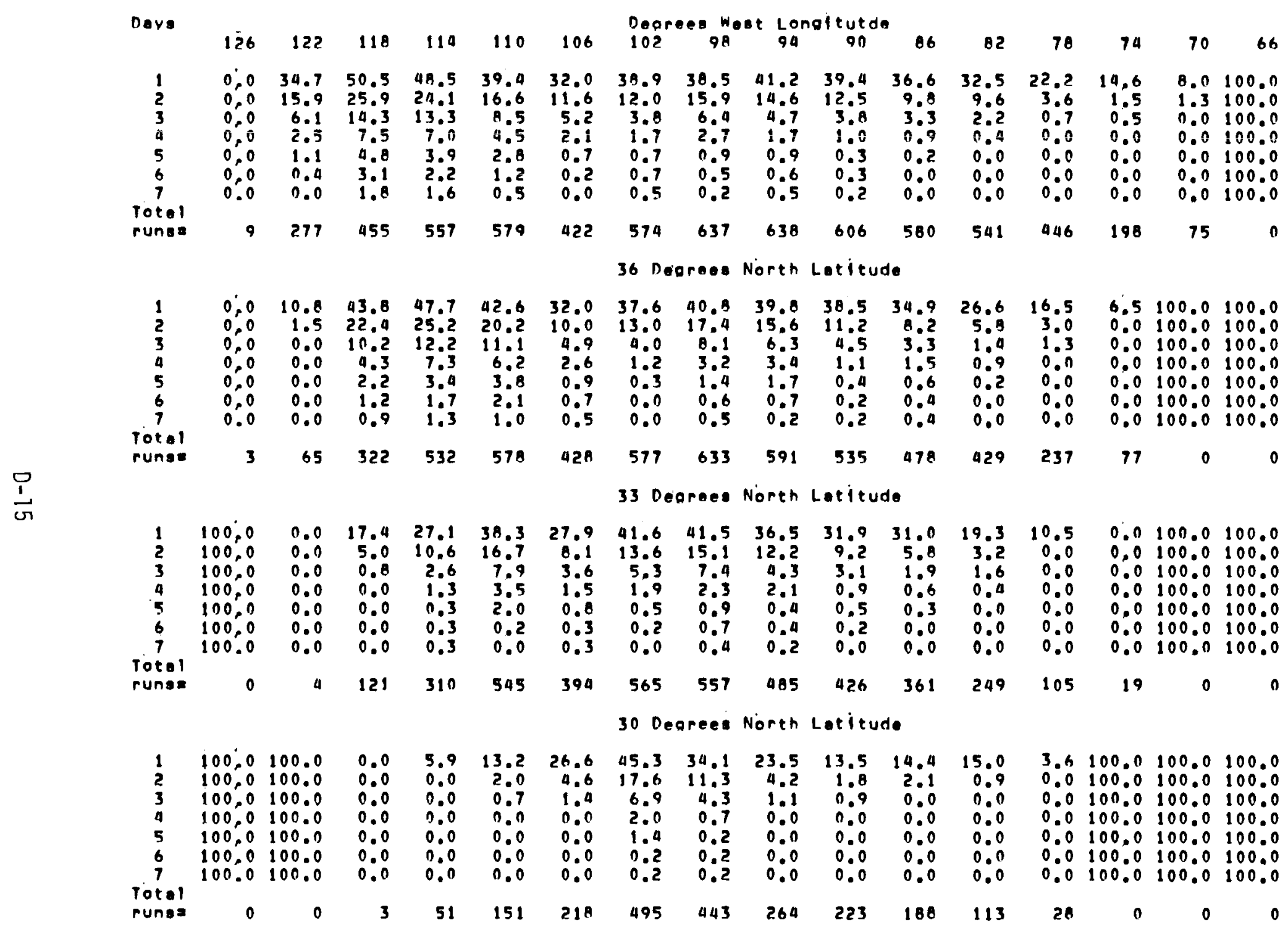


Probohility Run of WPT 5 Will Exceed Duration shown

\section{Deoroes North Lot ltude}

Dove

$\begin{array}{llllll}126 & 122 & 118 & 114 & 110 & 106\end{array}$

Degrees Wost Longitutde

1029696

82

7 月

74

70

66

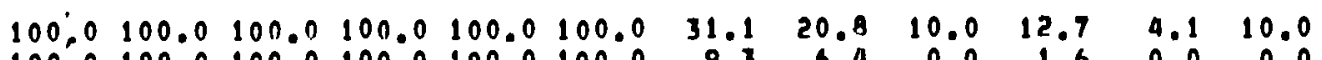

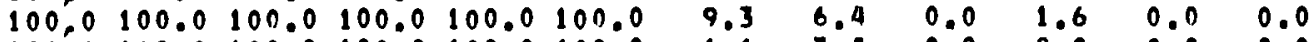
$100.0100 .0 \quad 100.0100 .0 \quad 100.0 \quad 100.0$ 100.0100 .0100 .0100 .0100 .0100 .0 100.0100 .0100 .0100 .0100 .0100 .0 100.0100 .0100 .0100 .0100 .0100 .0 100.0100 .0100 .0100 .0100 .0100 .0

$\begin{array}{llll}9.3 & 6.4 & 0.0 & 1.6 \\ 1.6 & 3.4 & 0.0 & 0.0\end{array}$

$\begin{array}{llll}1.6 & 3.4 & 0.0 & 0.0 \\ 0.3 & 0.7 & 0.0 & 0.0\end{array}$

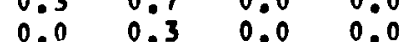

$0.0 \quad 0.0$

$\begin{array}{lll}0.0 & 0.0 & 0.0\end{array}$

$\begin{array}{llllll}0.0 & 0.3 & 0.0 & 0.0 & 0.0 & 0.0\end{array}$

0.0100 .0100 .0100 .0 $0.0 \quad 100.0 \quad 100.0100 .0$ 0.0100 .0100 .0100 .0 0.0100 .0100 .0100 .0 $0.0100 .0 \quad 100.0100 .0$ 0.0100 .0100 .0100 .0 0.0100 .0100 .0100 .0 Totol 0

$$
0
$$

$$
0
$$

$$
0
$$

24 Dearees North Latitude

$$
\begin{array}{r}
1 \\
2 \\
3 \\
4 \\
5 \\
6 \\
7 \\
\text { rot } \\
\text { run }
\end{array}
$$

$100.0100 .0 \quad 100.0 \quad 100.0 \quad 100.0 \quad 100.0 \quad 100.0$ 100.0100 .0100 .0100 .0100 .0100 .0100 .0 100,0100 .

18.7100 .0100 .0100 .0 3.9100 .0100 .0100 .0 1.5100 .0100 .0100 .0 $100.0 \quad 100.0 \quad 100.0 \quad 100.0 \quad 100.0100 .0100 .0$ 10.0 100.0 100.0 100.0 100.0100 .0100 .0 100.0100 .0100 .0100 .01100 .0100 .0100 .0 0.5100 .0100 .0100 .0 0.0100 .0100 .0100 .0 0.0100 .0100 .0100 .0 $0.0100 .0 \quad 100.0100 .0$$$
0.0
$$

30 7

$$
0 \quad 0
$$

$0.0 \quad 0.0 \quad 100.0 \quad 100.0100 .0$ $0.0 \quad 0.0 \quad 100.0 \quad 100.0100 .0$ $0.0 \quad 0.0100 .0100 .0100 .0$ $0.0 \quad 0.0100 .0100 .0100 .0$ $0.0 \quad 0.01000100 .0100 .0$ 0.01100 .0100 .0100 .0

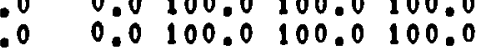
Totel 100.0100 .0100 .0100 .0100 .0100 .0100 .0 o

\begin{abstract}
0
\end{abstract}
$0 \quad 0$

$0 \quad 0$ 
51 Deprees North Latitude

Deye

$\begin{array}{lllll}126 & 122 \quad 118 & 114 & 110\end{array}$

$100.0100 .0 \quad 100.0 \quad 100.0 \quad 100.0$ 100.0100 .0100 .0100 .0100 .0 100.0100 .0100 .0100 .0100 .0 100.0100 .0100 .0100 .0100 .0 100.0100 .0100 .0100 .0100 .0 $100.0100 .0 \quad 100.0100 .0100 .0$

100.0100 .0100 .0100 .0100 .0

\section{rote}

Totel

Degreas Wat Lonaltutde

$\begin{array}{llllll}86 & 82 & 78 & 74 & 70 & 66\end{array}$

0.0100 .0100 .0100 .0100 .0100 .0100 .0100 .0100 .0100 .0100 .0 $0.0 \quad 100.0 \quad 100.0 \quad 100.0 \quad 100.0 \quad 100.0 \quad 100.0 \quad 100.0 \quad 100.0100 .0 \quad 100.0$ $0.0 \quad 100.0 \quad 100.0 \quad 100.0 \quad 100.0 \quad 100.0 \quad 100.0 \quad 100.0 \quad 100.0 \quad 100.0 \quad 100.0$ $0.0 \quad 100.0 \quad 100.0 \quad 100.0 \quad 100.0 \quad 100.0 \quad 100.0 \quad 100.0 \quad 100.0 \quad 100.0 \quad 100.0$ $0.0 \quad 100.0 \quad 100.0 \quad 100.0 \quad 100.0 \quad 100.0 \quad 100.0 \quad 100.0 \quad 100.0 \quad 100.0 \quad 100.0$ $0.0 \quad 100.0 \quad 100.0 \quad 100.0 \quad 100.0 \quad 100.0100 .0 \quad 100.0 \quad 100.0100 .0100 .0$ 0.0100 .0100 .0100 .0100 .0100 .0100 .0100 .0100 .0100 .0100 .0

\section{Degrees North Letitude}

100.0100 .0100 .0100 .0100 .0 100.0100 .0100 .0100 .0100 .0 100.0100 .0100 .0100 .0100 .0 100.0100 .0100 .0100 .0100 .0 100.0100 .0100 .0100 .0100 .0 100.0100 .0100 .0100 .0100 .0

100.0100 .0100 .0100 .0100 .0

rotal

$\begin{array}{rrrrrrrrrrr}0.0 & 100.0 & 0.0 & 25.0 & 18.8 & 19.4 & 23.7 & 25.5 & 16.2 & 20.2 & 18.0 \\ 0.0 & 100.0 & 0.0 & 0.0 & 12.5 & 3.2 & 5.3 & 4.3 & 1.5 & 1.0 & 1.0 \\ 0.0 & 100.0 & 0.0 & 0.0 & 0.0 & 0.0 & 0.0 & 0.0 & 0.0 & 1.0 & 0.7 \\ 0.0 & 100.0 & 0.0 & 0.0 & 0.0 & 0.0 & 0.0 & 0.0 & 0.0 & 0.0 & 0.0 \\ 0.0 & 100.0 & 0.0 & 0.0 & 0.0 & 0.0 & 0.0 & 0.0 & 0.0 & 0.0 & 0.0 \\ 0.0 & 100.0 & 0.0 & 0.0 & 0.0 & 0.0 & 0.0 & 0.0 & 0.0 & 0.0 & 0.0 \\ 0.0 & 100.0 & 0.0 & 0.0 & 0.0 & 0.0 & 0.0 & 0.0 & 0.0 & 0.0 & 0.0 \\ 1 & 0 & 2 & 8 & 16 & 31 & 38 & 97 & 68 & 99 & 139\end{array}$

45 Deprees North Latieude

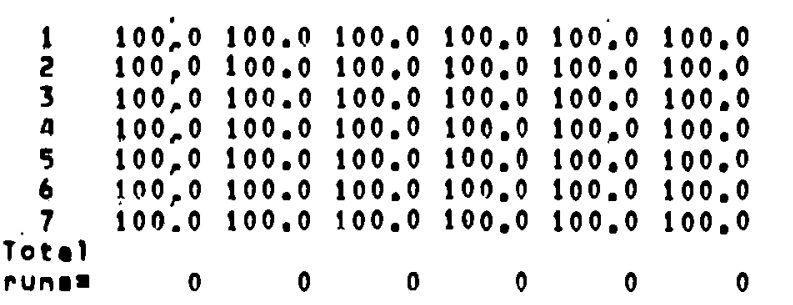

$\begin{array}{rrrrrrrrrr}0.0 & 6.7 & 32.4 & 23.9 & 32.9 & 31.7 & 30.1 & 25.2 & 19.4 & 22.7 \\ 0.0 & 0.0 & 10.8 & 9.0 & 11.0 & 9.8 & 7.5 & 4.7 & 5.0 & 6.2 \\ 0.0 & 0.0 & 0.0 & 1.5 & 4.1 & 4.9 & 2.2 & 1.6 & 0.6 & 1.7 \\ 0.0 & 0.0 & 0.0 & 0.0 & 2.7 & 2.4 & 2.2 & 0.8 & 0.0 & 0.4 \\ 0.0 & 0.0 & 0.0 & 0.0 & 0.0 & 1.2 & 1.1 & 0.0 & 0.0 & 0.0 \\ 0.0 & 0.0 & 0.0 & 0.0 & 0.0 & 0.0 & 0.0 & 0.0 & 0.0 & 0.0 \\ 0.0 & 0.0 & 0.0 & 0.0 & 0.0 & 0.0 & 0.0 & 0.0 & 0.0 & 0.0 \\ 3 & 15 & 37 & 67 & 73 & 82 & 93 & 127 & 180 & 242 \\ 42 & \text { Deoreo. North Letitude } & & & & & \end{array}$
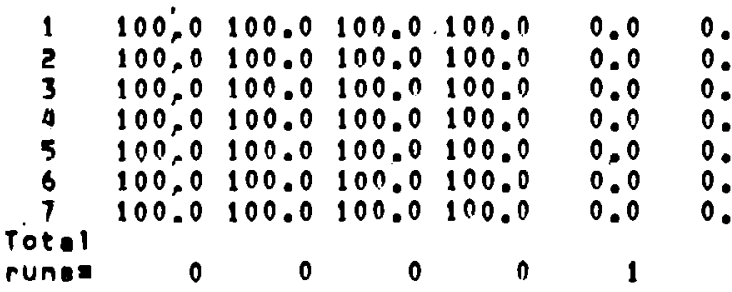

$\begin{array}{rrrrrrrrrrr}0.0 & 30.0 & 27.1 & 39.1 & 38.5 & 38.6 & 35.9 & 32.2 & 27.1 & 28.2 & 31.7 \\ 0.0 & 10.0 & 12.5 & 19.9 & 17.7 & 15.2 & 14.4 & 11.5 & 12.6 & 10.5 & 12.1 \\ 0.0 & 0.0 & 4.2 & 3.4 & 6.2 & 7.6 & 9.2 & 0.4 & 4.2 & 3.4 & 4.3 \\ 0.0 & 0.0 & 0.0 & 0.0 & 3.1 & 4.8 & 3.9 & 1.6 & 0.9 & 1.7 & 1.7 \\ 0.0 & 0.0 & 0.0 & 0.0 & 0.0 & 0.7 & 1.3 & 0.5 & 0.0 & 1.0 & 1.2 \\ 0.0 & 0.0 & 0.0 & 0.0 & 0.0 & 0.0 & 1.3 & 0.5 & 0.0 & 0.3 & 0.9 \\ 0.0 & 0.0 & 0.0 & 0.0 & 0.0 & 0.0 & 0.0 & 0.0 & 0.0 & 0.3 & 0.6 \\ 4 & 10 & 48 & 87 & 130 & 145 & 153 & 183 & 214 & 294 & 347\end{array}$


39 Degreas North Letitude

Dava

$126 \quad 122 \quad 110 \quad 114$

$100,0 \quad 100.0 \quad 100.0 \quad 100.0$ $100,0100.0100 .0100 .0$ $100,0 \quad 100.0 \quad 100.0100 .0$ $100,0100.0100 .0100 .0$ $100.0100 .0 \quad 100.0100 .0$ $100.0100 .0100 .0 \quad 100.0$ 100.0100 .0100 .0100 .0 Totel runge

$0 \quad 0 \quad 0 \quad 0$

$100.0 \quad 100.0 \quad 100.0 \quad 100.0$ $100.0 \quad 100.0 \quad 100.0100 .0$ $100.0 \quad 100.0 \quad 100.0 \quad 100.0$ $100.0 \quad 100.0 \quad 100.0100 .0$ 100.100 .0100 .0100 .0 100.000 .0100 .0100 .0 Totol 100.0100 .0100 .0100 .0

1
0
0
0
0
0
0
0


0
0
0
0
0
0
0

Degreeg West Longleutde

$110 \quad 106$

$0.0 \quad 14.3$

989490

$0.0 \quad 14.3$

$\begin{array}{rrrrrrrrrr}17.6 & 31.8 & 50.3 & 48.7 & 49.8 & 43.8 & 33.3 & 32.7 & 36.2 & 100.0 \\ 5.9 & 14.4 & 26.2 & 27.6 & 26.8 & 23.9 & 13.3 & 13.7 & 14.3 & 100.0 \\ 5.9 & 5.3 & 9.2 & 13.8 & 13.8 & 9.1 & 7.0 & 6.3 & 6.6 & 100.0 \\ 0.0 & 3.0 & 5.1 & 7.3 & 7.9 & 4.7 & 2.9 & 3.6 & 3.6 & 100.0 \\ 0.0 & 1.5 & 2.1 & 3.4 & 4.6 & 2.5 & 1.6 & 1.5 & 2.0 & 100.0 \\ 0.0 & 0.8 & 1.5 & 2.6 & 3.3 & 1.1 & 1.0 & 0.8 & 1.3 & 100.0 \\ 0.0 & 0.0 & 1.0 & 1.3 & 2.1 & 1.1 & 1.0 & 0.5 & 0.9 & 100.0 \\ 34 & 132 & 195 & 232 & 239 & 276 & 315 & 394 & 442 & 0\end{array}$

36 Deprees North Letitude

0.0
0.0
0.0
0.0
0.0
0.0
0.0

5.6
5.6
0.0
0.0
0.0
0.0
0.0
18

$\begin{array}{rrrrr}25.0 & 47.8 & 53.6 & 55.7 & 50.3 \\ 13.8 & 25.7 & 35.4 & 34.5 & 31.7 \\ 6.9 & 16.8 & 24.6 & 23.2 & 22.5 \\ 4.3 & 11.6 & 18.5 & 14.8 & 15.6 \\ 0.9 & 7.3 & 12.3 & 11.0 & 11.9 \\ 0.9 & 3.4 & 6.6 & 6.4 & 6.3 \\ 0.9 & 2.2 & 5.0 & 5.8 & 6 . \\ 116 & 268 & 302 & 345 & 36\end{array}$

33 Deprees North Letitude

$100 \% 0 \quad 100.0 \quad 100.0$ $100.0 \quad 100.0 \quad 100.0$ $100.0100 .0 \quad 100.0$ $100.0100 .0 \quad 100.0$ 100.01100 .01100 .0 100.01107 .0100 .0 $\begin{array}{lll}100,0 & 100.0 & 100.0 \\ 100.0 & 100.0 & 100.0\end{array}$

100.0100 .0100 .0100 .0 $100,0100.0 \quad 100.0100 .0$ $100,0100.0100 .0100 .0$ 100.0100 .0100 .0100 .0 100.0100 .0100 .0100 .0 $100,0 \quad 100.0 \quad 100.0 \quad 100.0$ 100.0100 .0100 .0100 .0

0.0
0.0
0.0
0.0
0.0
0.0
0.0
1
0.0
0
.0
.0
0.0
00
0

$14.3 \quad 10.5$

$\begin{array}{rrrrrrrrrrrr}14.3 & 10.5 & 35.0 & 50.4 & 52.6 & 55.2 & 54.8 & 53.7 & 53.7 & 54.5 & 100.0 & 100.0 \\ 0.0 & 7.9 & 14.4 & 31.7 & 35.5 & 36.3 & 35.8 & 32.3 & 32.4 & 34.4 & 100.0 & 100.0 \\ 0.0 & 0.0 & 7.4 & 21.7 & 26.1 & 25.2 & 24.5 & 22.2 & 19.3 & 20.49 & 100.0 & 100.0 \\ 0.0 & 0.0 & 5.3 & 15.7 & 18.0 & 19.2 & 18.8 & 15.2 & 11.0 & 11.7 & 100.0 & 100.0 \\ 0.0 & 0.0 & 4.1 & 11.7 & 13.5 & 14.1 & 13.1 & 11.0 & 8.4 & 8.1 & 100.0 & 100.0 \\ 0.0 & 0.0 & 2.5 & 8.5 & 12.3 & 12.2 & 12.2 & 7.8 & 6.1 & 5.5 & 100.0 & 100.0 \\ 0.0 & 0.0 & 1.6 & 7.2 & 11.4 & 10.9 & 9.4 & 5.1 & 4.2 & 4.0 & 100.0 & 100.0 \\ 7 & 38 & 2.43 & 401 & 422 & 433 & 458 & 527 & 592 & 631 & 0 & 0\end{array}$
30 Deprees North Letitude

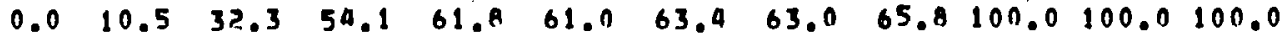

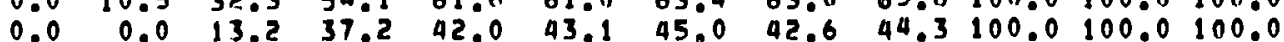

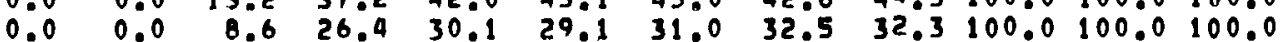
$0.0 \quad 0.0 \quad 4.6 \quad 18.2 \quad 21.7 \quad 23.2 \quad 24.4 \quad 24.0 \quad 22.7100 .0100 .0100 .0$

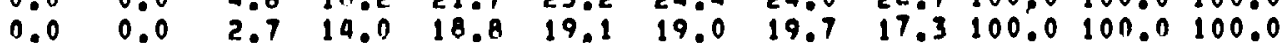

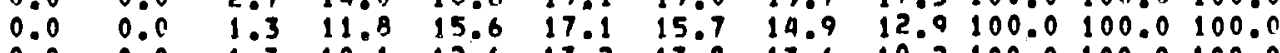
$0.0 \quad 0.0 \quad 1.3 \quad 10.1 \quad 12.6 \quad 13.2 \quad 13.8 \quad 13.6 \quad 10.2100 .0100 .0100 .0$

\begin{abstract}
372
\end{abstract}
$473469 \quad 508$

13.6 
Probability Run of MPT 6 will Exceod nuration shown

27 Dearees North Latitude

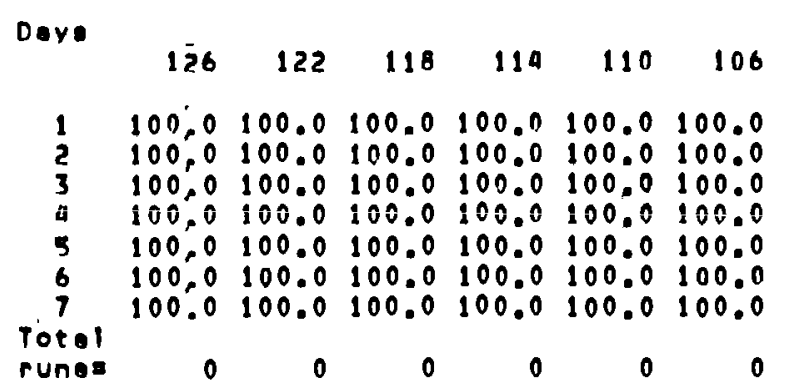

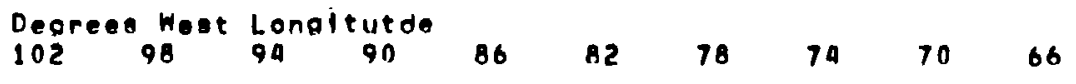

$\begin{array}{llllllllll}25.6 & 59.7 & 68.9 & 72.6 & 74.2 & 73.9 & 73.0 & 100.0 & 100.0 & 100.0\end{array}$

$\begin{array}{llllllllll}7.4 & 42.6 & 49.7 & 55.9 & 55.4 & 54.3 & 55.1 & 100.0 & 100.0 & 100.0\end{array}$

$2.4 \quad 29.7 \quad 38.6 \quad 40.6 \quad 42.7 \quad 43.6 \quad 44.2 \quad 100.0 \quad 100.0 \quad 100.0$

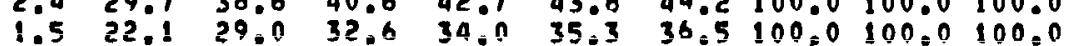

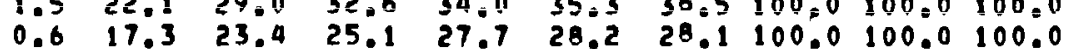

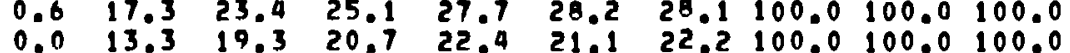

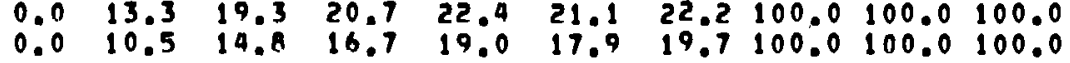

24 Deorees North Lotitude

$100.0 \quad 100.0 \quad 100.0 \quad 100.0 \quad 100.0 \quad 100.0 \quad 100.0 \quad 58.2 \quad 100.0 \quad 100.0 \quad 100.0 \quad 76.4 \quad 75.9100 .0100 .0100 .0$ $100.0100 .0100 .0100 .0100 .0100 .0100 .0 \quad 37.5100 .01100 .01100 .0$ $100.0 \quad 100.0100 .0100 .0100 .0100 .0100 .0 \quad 24.7100 .0100 .0100 .0 \quad 51.6 \quad 49.4100 .0100 .0100 .0$ $100.0100 .0100 .0100 .0100 .0100 .0100 .0 \quad 17.8100 .0100 .0100 .0 \quad 42.540 .3100 .0100 .0100 .0$ $100.000 .0100 .0100 .0100 .0100 .0100 .0 \quad 12.9100 .0100 .0100 .0 \quad 34.6 \quad 32.3100 .0100 .0100 .0$ $100.0100 .0100 .01100 .0100 .0100 .0100 .0 \quad 12.9100 .0100 .0100 .0 \quad 34.6 \quad 32.3100 .0100 .0100 .0$ 8.9100 .0100 .0100 .028 .328 .3100 .01100 .0100 .0 $100.0100 .0100 .0100 .0100 .0100 .0100 .0 \quad 6.7100 .0100 .0100 .0 \quad 24.1 \quad 24.3100 .0100 .0100 .0$ Totel 
Probobility Run of WPT 7 Will Exceed Duretion shown

51 Deorees North Letitude

\begin{tabular}{|c|c|c|c|c|c|c|c|c|c|c|c|c|c|c|c|c|}
\hline Deve & 126 & 122 & 118 & 114 & 110 & 106 & $\begin{array}{l}\text { Deq } \\
102\end{array}$ & 98 & $t \operatorname{lion}_{94}$ & $\begin{array}{c}\text { itutd } \\
\text { 9n }\end{array}$ & 86 & 82 & 78 & 74 & 70 & 66 \\
\hline $\begin{array}{l}1 \\
2 \\
3 \\
4 \\
5 \\
6 \\
7\end{array}$ & $\begin{array}{r}17,4 \\
2,6 \\
1,1 \\
0,5 \\
0,5 \\
0,5 \\
0.5\end{array}$ & $\begin{array}{l}20.5 \\
5.6 \\
2.6 \\
2.1 \\
0.4 \\
0.4 \\
0.4\end{array}$ & $\begin{array}{r}29.9 \\
10.6 \\
4.5 \\
2.4 \\
2.1 \\
1.5 \\
1.5\end{array}$ & $\begin{array}{r}36.0 \\
16.5 \\
4.0 \\
2.5 \\
1.3 \\
0.0 \\
0.0\end{array}$ & $\begin{array}{r}25.1 \\
8.4 \\
2.6 \\
0.8 \\
0.0 \\
0.0 \\
0.0\end{array}$ & $\begin{array}{l}23.9 \\
6.1 \\
1.5 \\
0.6 \\
0.0 \\
0.0 \\
0.0\end{array}$ & $\begin{array}{l}21.8 \\
3.2 \\
0.4 \\
0.0 \\
0.0 \\
0.0 \\
0.0\end{array}$ & $\begin{array}{r}18.5 \\
3.8 \\
1.3 \\
0.4 \\
0.4 \\
0.4 \\
0.0\end{array}$ & $\begin{array}{l}16.6 \\
4.5 \\
2.5 \\
1.0 \\
0.5 \\
0.0 \\
0.0\end{array}$ & $\begin{array}{l}100.0 \\
100.0 \\
100.0 \\
100.0 \\
100.0 \\
100.0 \\
100.0\end{array}$ & $\begin{array}{l}100.0 \\
100.0 \\
100.0 \\
100.0 \\
100.0 \\
100.0 \\
100.0\end{array}$ & $\begin{array}{l}100.0 \\
100.0 \\
100.0 \\
100.0 \\
100.0 \\
100.0 \\
100.0\end{array}$ & $\begin{array}{l}100.0 \\
100.0 \\
100.0 \\
100.0 \\
100.0 \\
100.0 \\
100.0\end{array}$ & $\begin{array}{l}100.0 \\
100.0 \\
100.0 \\
100.0 \\
100.0 \\
100.0 \\
100.0\end{array}$ & $\begin{array}{l}100.0 \\
100.0 \\
100.0 \\
100.0 \\
100.0 \\
100.0 \\
100.0\end{array}$ & $\begin{array}{l}1000.0 \\
1000.0 \\
100.0 \\
100.0 \\
100.0 \\
100.0 \\
100.0\end{array}$ \\
\hline
\end{tabular}

48 Degrees Noreh Letitude

$\begin{array}{crrrrrr}1 & 11.9 & 13.4 & 22.6 & 27.8 & 34.1 & 23.2 \\ ? & 2.3 & 2.5 & 5.1 & 11.5 & 13.2 & 7.0 \\ 3 & 0.0 & 0.0 & 3.4 & 5.0 & 5.6 & 2.3 \\ 4 & 0.0 & 0.0 & 2.4 & 3.4 & 2.7 & 0.5 \\ 5 & 0.0 & 0.0 & 0.0 & 1.6 & 1.5 & 0.0 \\ 6 & 0.0 & 0.0 & 0.0 & 0.5 & 0.7 & 0.0 \\ 7 & 0.0 & 0.0 & 0.0 & 0.5 & 0.5 & 0.0 \\ \text { Totol } & 177 & 202 & 296 & 381 & 410 & 383 \\ \text { runes } & 177 & & & \end{array}$

$\begin{array}{rrrrrrrrrr}24.9 & 17.5 & 13.5 & 16.6 & 12.9 & 12.9 & 15.8 & 17.8 & 20.5 & 19.8 \\ 5.1 & 3.4 & 2.9 & 2.0 & 2.2 & 1.7 & 2.9 & 1.4 & 2.6 & 4.3 \\ 0.6 & 0.7 & 2.0 & 0.5 & 0.5 & 0.6 & 0.0 & 0.0 & 1.3 & 0.6 \\ 0.0 & 0.3 & 0.4 & 0.0 & 0.0 & 0.0 & 0.0 & 0.0 & 0.6 & 0.0 \\ 0.0 & 0.3 & 0.0 & 0.0 & 0.0 & 0.0 & 0.0 & 0.0 & 0.0 & 0.0 \\ 0.0 & 0.3 & 0.0 & 0.0 & 0.0 & 0.0 & 0.0 & 0.0 & 0.0 & 0.0 \\ 0.0 & 0.0 & 0.0 & 0.0 & 0.0 & 0.0 & 0.0 & 0.0 & 0.0 & 0.0 \\ 333 & 291 & 245 & 205 & 186 & 170 & 171 & 146 & 156 & 162\end{array}$

45 Deorees North Lotitude

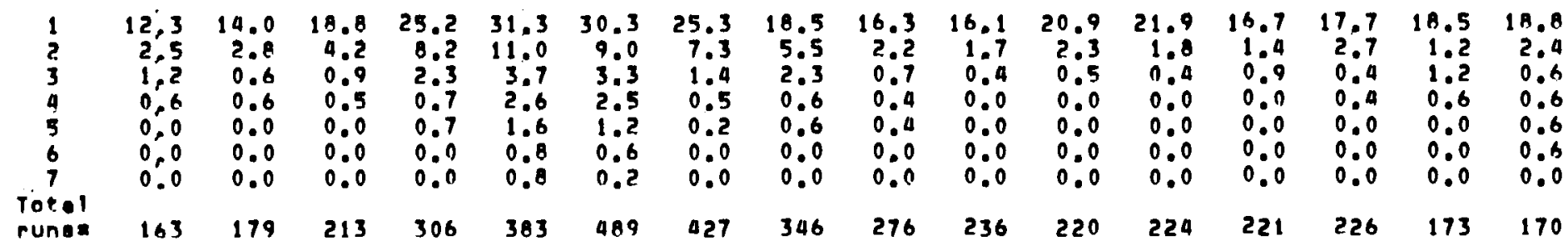

42 Degrees North Lotitude

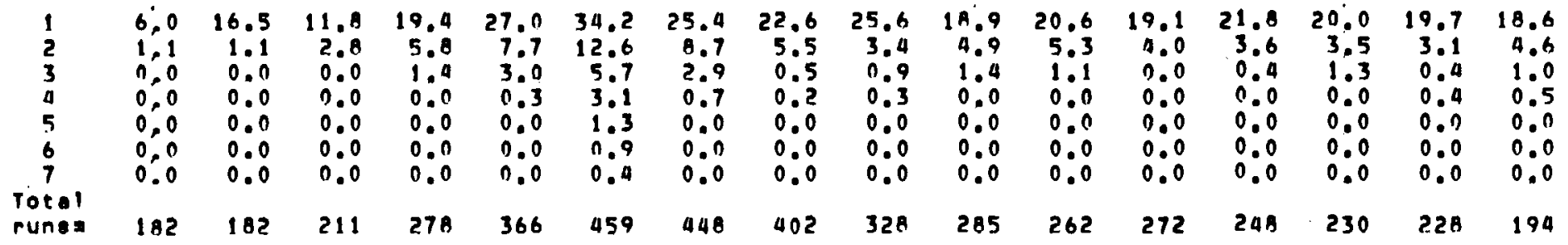


Probability Run of KPT 7 Will Exceed Duration shown

39 Degreas North Latitude

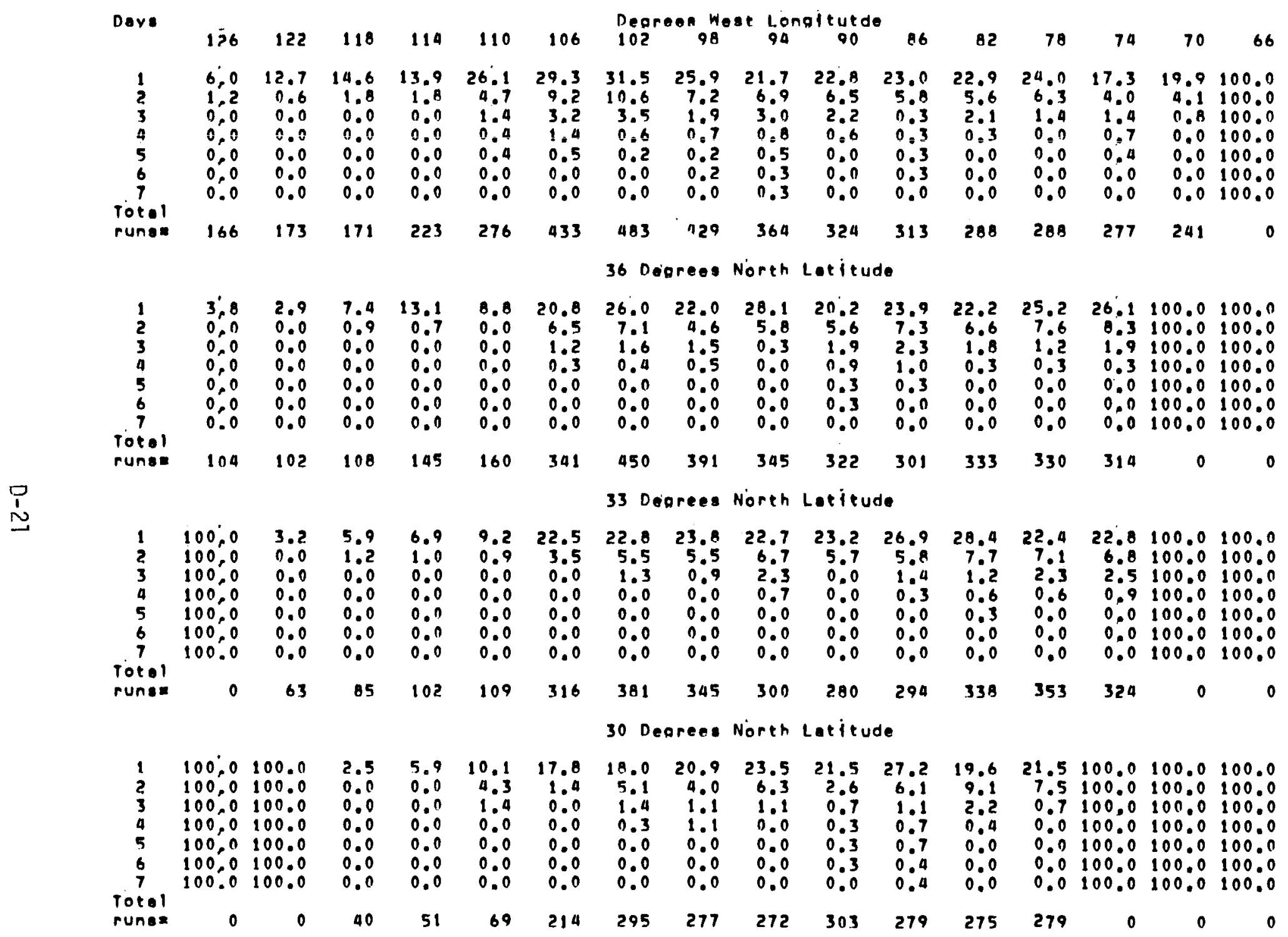


27 nograse North Lintitude

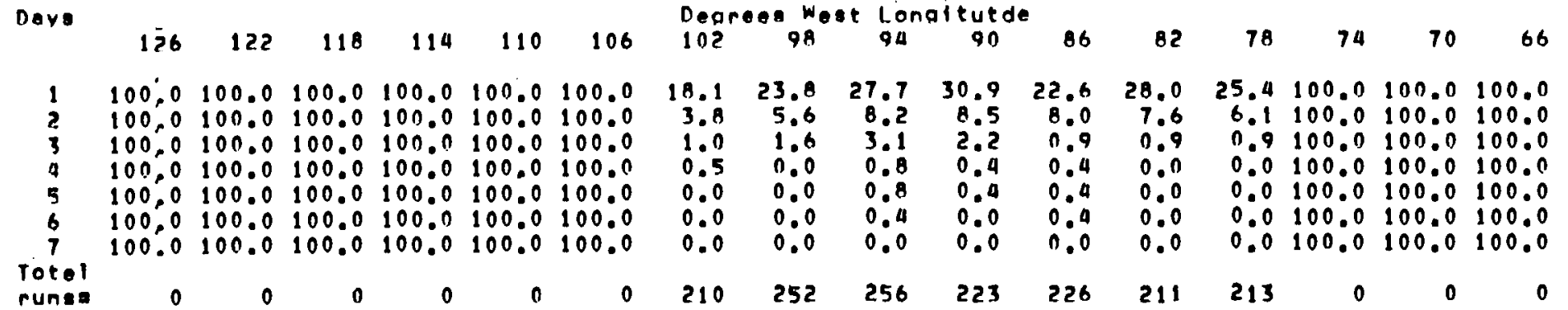

24 Doreos North Lotitude

$100.0100 .0100 .0100 .0100 .0100 .0 \quad 100.0 \quad 23.2100 .0100 .0100 .0 \quad 29.921 .7100 .0100 .0100 .0$ $100.0100 .0 \quad 100.0 \quad 100.0 \quad 100.0 \quad 100.0 \quad 100.0 \quad 5.2 \quad 100.0100 .0100 .0 \quad 0.7 \quad 6.3 \quad 100.0100 .0100 .0$ $100.0100 .0 \quad 100.0 \quad 100.0 \quad 100.0 \quad 100.0 \quad 100.0 \quad 1.3100 .0100 .0100 .0$ $100.0100 .0 \quad 100.0 \quad 100.0100 .0 \quad 100.0 \quad 100.0 \quad 0.6 \quad 100.0100 .0100 .0$

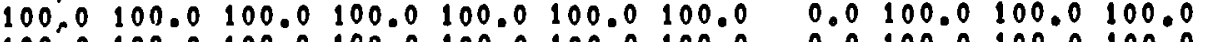
$100.0100 .0 \quad 100.0 \quad 100.0 \quad 100.0 \quad 100.0 \quad 100.0 \quad 0.0100 .0100 .0100 .0$ 7100.0100 .0100 .0100 .0100 .0100 .0100 .0 
Probebility Run of WPT will Exceed Duration shown

51 Deareas North Latitude

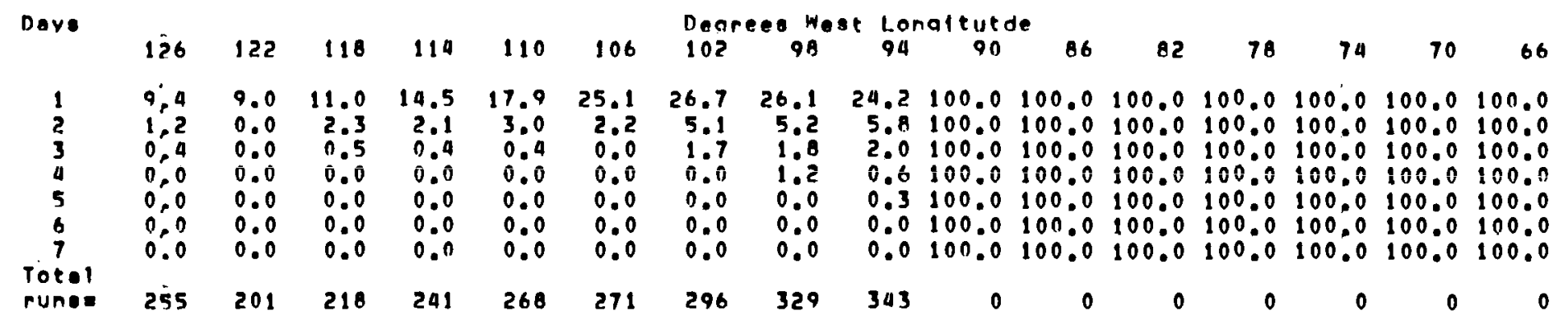

4 Begrees North Lotitude

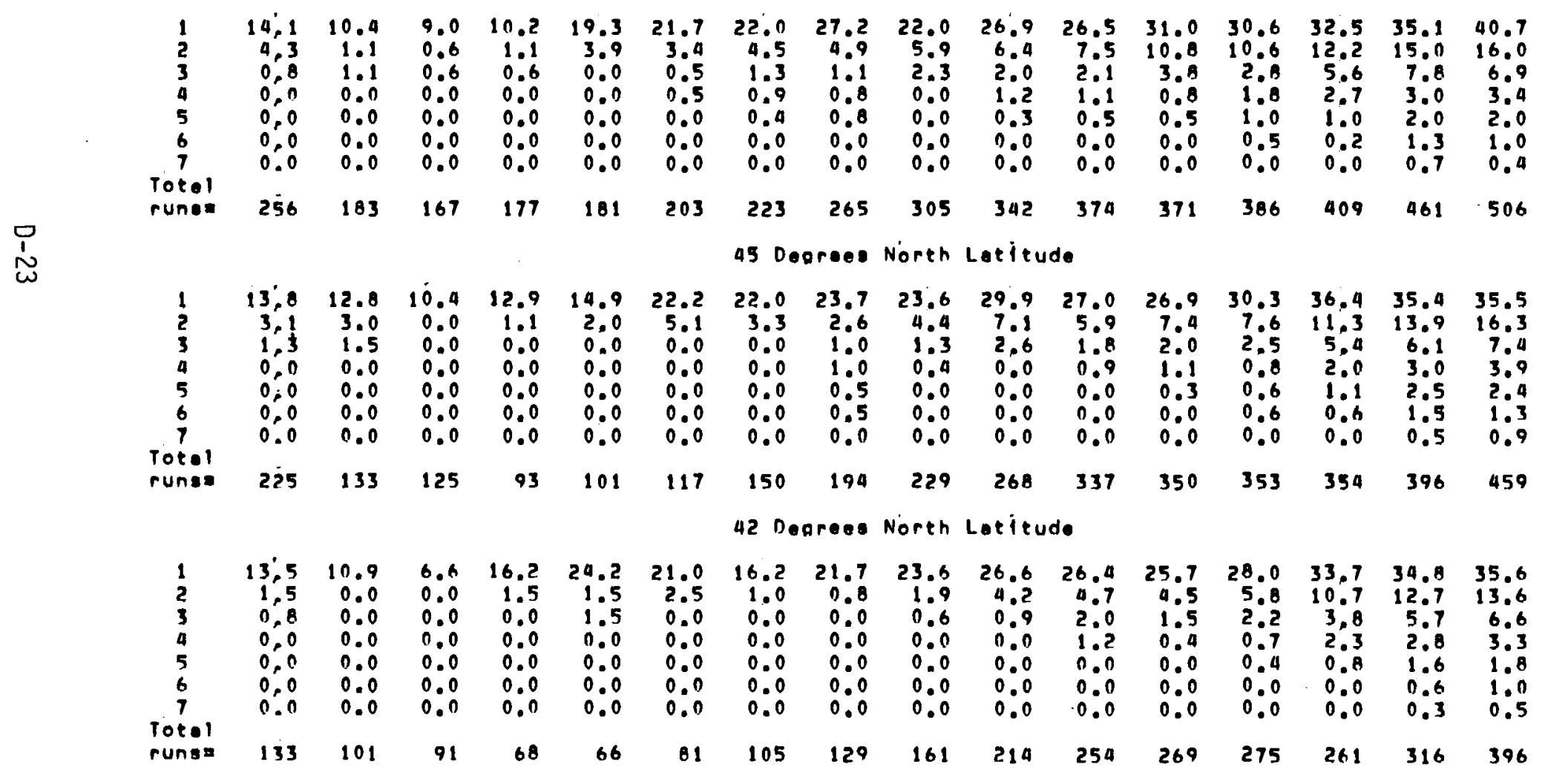


39 Deprees North l.atitude




Probobility Run of NPT B Will Exceed Duration shown

27 Deorees North Lotitude

\begin{tabular}{|c|c|c|c|c|c|c|c|c|c|c|c|c|c|c|c|c|}
\hline Dave & 126 & 122 & 118 & 114 & 110 & 106 & $\begin{array}{l}\text { negr } \\
102\end{array}$ & ex West & $\operatorname{Lon}_{94}$ & $\begin{array}{c}\text { itutde } \\
90\end{array}$ & 86 & 82 & 78 & 74 & 70 & 66 \\
\hline $\begin{array}{l}1 \\
2 \\
3 \\
4 \\
5 \\
6 \\
7\end{array}$ & $\begin{array}{l}100,0 \\
100,0 \\
100,0 \\
100,0 \\
100,0 \\
100,0 \\
100.0\end{array}$ & $\begin{array}{l}100.0 \\
100.0 \\
100.0 \\
100.0 \\
100.0 \\
100.0 \\
100.0\end{array}$ & $\begin{array}{l}100.0 \\
100.0 \\
100.0 \\
100.0 \\
100.0 \\
100.0 \\
100.0\end{array}$ & $\begin{array}{l}1000.0 \\
1000.0 \\
1000.0 \\
1000.0 \\
1000.0 \\
1000.0 \\
100.0\end{array}$ & $\begin{array}{l}100.0 \\
1000.0 \\
100.0 \\
100.0 \\
100.0 \\
100.0 \\
100.0\end{array}$ & $\begin{array}{l}100.0 \\
100.0 \\
100.0 \\
10000 \\
100.0 \\
100.0 \\
100.0\end{array}$ & $\begin{array}{l}100.0 \\
100.0 \\
100.0 \\
100.0 \\
100.0 \\
100.0 \\
100.0\end{array}$ & $\begin{array}{ll}0 . & 0 \\
0.0 & 0 \\
0.0 & 0 \\
0.0 & 0 \\
0.0 & 0 \\
0.0 & 0 \\
0.0 & 0\end{array}$ & $\begin{array}{l}0.0 \\
0.0 \\
0.0 \\
0.0 \\
0.0 \\
0.0 \\
0.0\end{array}$ & $\begin{array}{l}0.0 \\
0.0 \\
0.0 \\
0.0 \\
0.0 \\
0.0 \\
0.0\end{array}$ & $\begin{array}{l}10000 \\
100.0 \\
100.0 \\
100.0 \\
100.0 \\
10000 \\
100.0\end{array}$ & $\begin{array}{l}0.0 \\
0.0 \\
0.0 \\
0.0 \\
0.0 \\
0.0 \\
0.0\end{array}$ & $\begin{array}{l}0.0 \\
0.0 \\
0.0 \\
0.0 \\
0.0 \\
0.0 \\
0.0\end{array}$ & $\begin{array}{l}100.0 \\
100.0 \\
100.0 \\
100.0 \\
100.0 \\
100.0 \\
100.0\end{array}$ & $\begin{array}{l}100.0 \\
100.0 \\
100.0 \\
100.0 \\
100.0 \\
100.0 \\
100.0\end{array}$ & $\begin{array}{l}100.0 \\
100.0 \\
100.0 \\
10000 \\
10000 \\
100.0 \\
100.0\end{array}$ \\
\hline runas & 0 & 0 & 0 & 0 & 0 & 0 & 0 & 2 & 1 & 1 & 0 & 1 & 2 & 0 & 0 & 0 \\
\hline
\end{tabular}

24 Degrees North Lotitude

ज̃

$100,0100.0 \quad 100.0100 .0100 .0100 .0100 .0100 .0100 .0100 .0100 .0100 .0100 .0100 .0100 .0100 .0$ $100,0100.0100 .0100 .0 \quad 100.0100 .0 \quad 100.0 \quad 100.0100 .0100 .0100 .0100 .0100 .0100 .0100 .0100 .0$ $100.0 \quad 100.0100 .0100 .0100 .0 \quad 100.0 \quad 100.0100 .0 \quad 100.0100 .0100 .0100 .0100 .0100 .0100 .0100 .0$

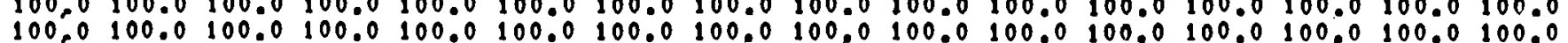
$100,0 \quad 100.0100 .0 \quad 100.0 \quad 100.0 \quad 100.0 \quad 100.0100 .0100 .0100 .0100 .0100 .0100 .0100 .0100 .0100 .0$ $100.0 \quad 100.0100 .0100 .0 \quad 100.0100 .0 \quad 100.0100 .0 \quad 100.0100 .0 \quad 100.0100 .0100 .0100 .0100 .0100 .0$ 100.0100 .0100 .0100 .0100 .0100 .0100 .0100 .0100 .0100 .0100 .0100 .0100 .0100 .0100 .0100 .0 rotal

0

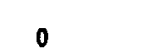

0

0

0


51 Dogrees North Lotitude

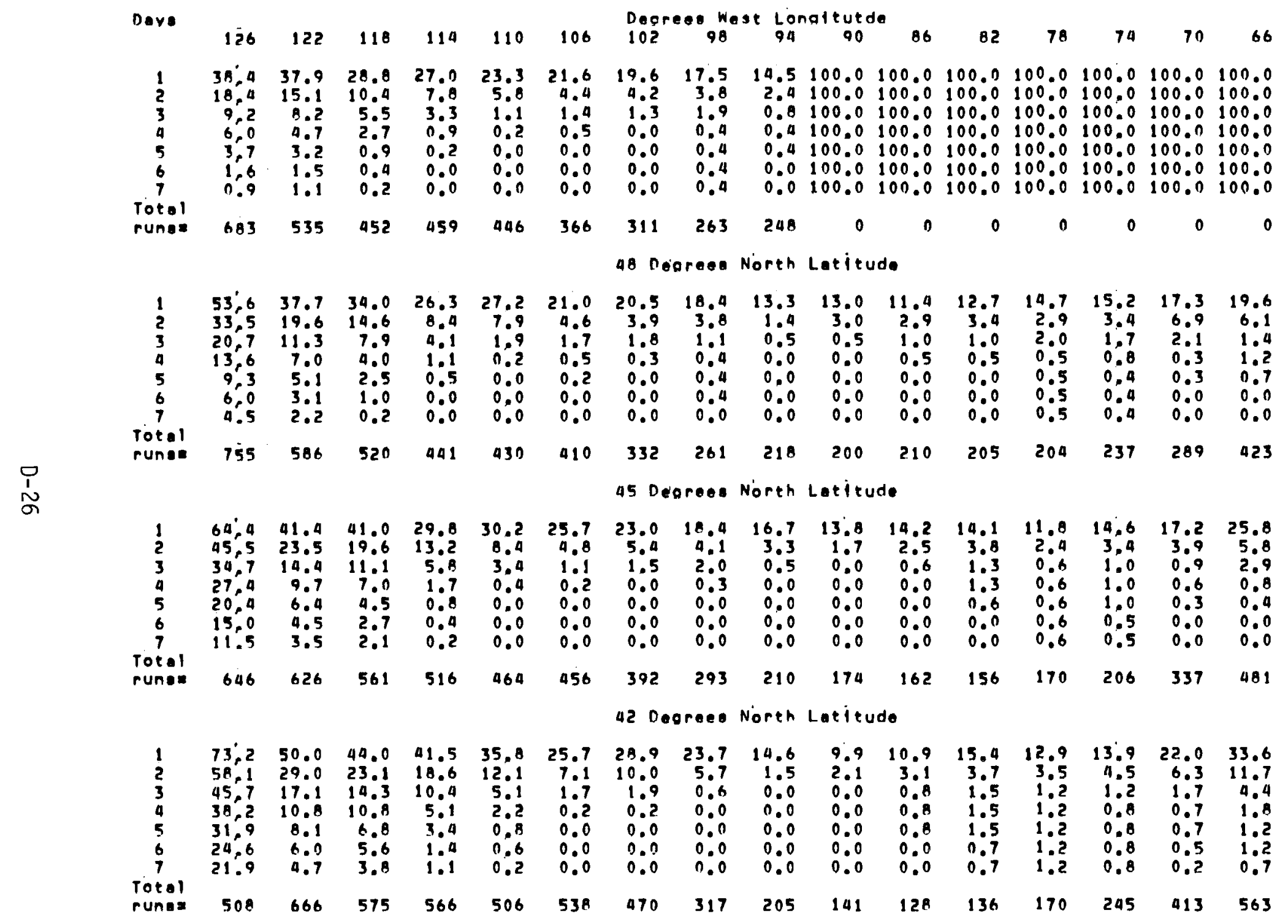


Probability Run of HPT 9 Will Exceed Durotion shown

39 Degrees North Lotitude

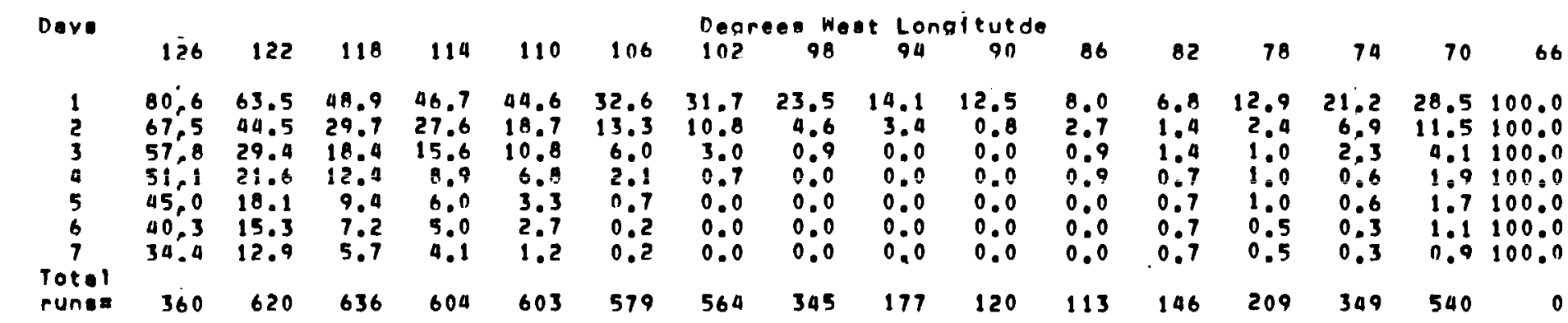

36 Degroes North Letitude

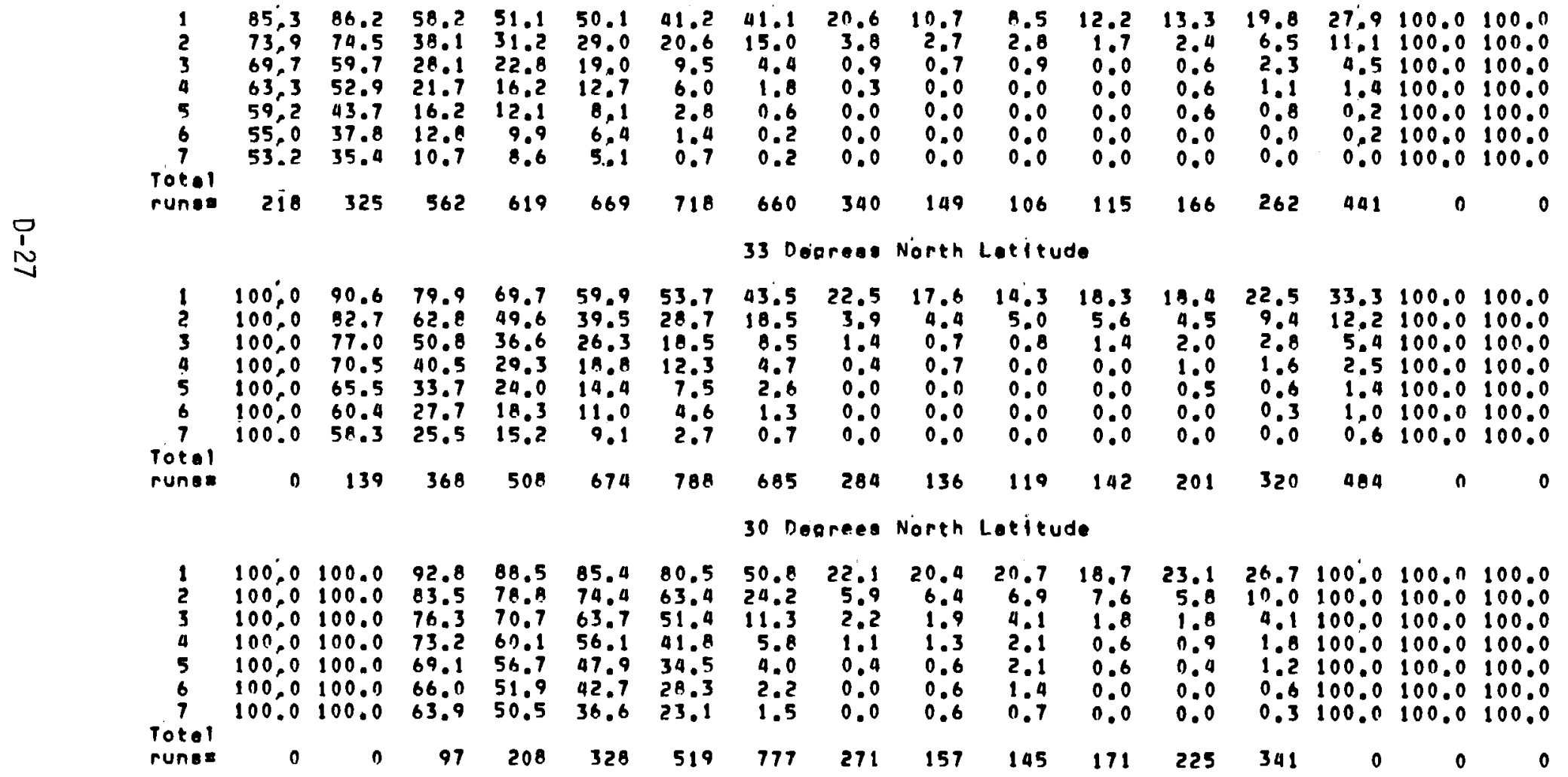


Probability Run of WPT 9 Will Exceod Duration shown

27 Doprese North Latitude

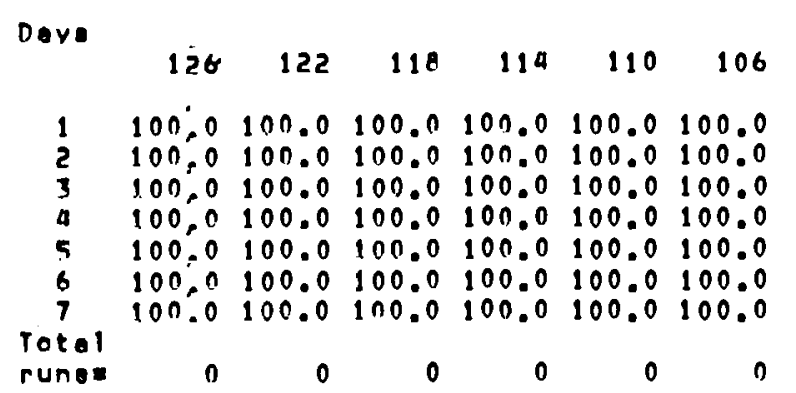

Degrees Woot Longltutde

$10298 \quad 94 \quad 90 \quad 86$

$\begin{array}{lllll}67.4 & 24.2 & 22.6 & 24.4 & 27.9\end{array}$

$46.1 \quad 6.0 \quad 8.8 \quad 10.9 \quad 10.9$

30.93 .0

22.6

1.1

1.1

8.8
4.4
1.8

1.83.

$3.2 \quad 2.2$

17.

0.5

1.3

$1.8 \quad 0.9$

0.9

82

78

74

70

66

$29.6 \quad 32.6 \quad 100.0 \quad 100.0 \quad 100.0$

$12.6 \quad 13.5 \quad 100.0 \quad 100.0100 .0$

$6.1 \quad 6.1100 .0100 .0100 .0$

$4.3 \quad 2.9100 .0 \cdot 100.0100 .0$

$1.9100 .0100 .0 \quad 100.0$

1.0100 .0100 .0100 .0

$\begin{array}{lllll}699 & 368 \quad 226 \quad 221 \quad 229\end{array}$

$230 \quad 310$

24 Deorees North Latitudo

$100.0100 .0 \quad 100.0 \quad 100.0 \quad 100.0100 .0 \quad 100.0$ $100.0100 .0 \quad 100.0100 .0100 .0100 .0100 .0$ $100.0 \quad 100.0 \quad 100.0 \quad 100.0 \quad 100.0100 .0100 .0$ $100.0100 .0 \quad 100.0 \quad 100.0 \quad 100.0 \quad 100.0100 .0$ 100.0100 .0100 .0100 .0100 .0100 .0100 .0 100.0100 .0100 .0100 .0100 .0100 .0100 .0

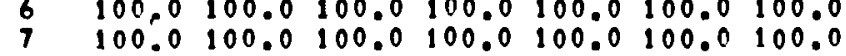

35.4100 .0100 .0100 .0

$30.6 \quad 35.3100 .0100 .0100 .0$ $5.8 \quad 100.0100 .0 \quad 100.0 \quad 7.4 \quad 6.1100 .0 \quad 100.0 \quad 100.0$ $2.5100 .0100 .0100 .0 \quad 4.5 \quad 2.6100 .0100 .0100 .0$ $1.3100 .0100 .0100 .0 \quad 2.6 \quad 1.4100 .0100 .0100 .0$ $1.2100 .0100 .0100 .0 \quad 1.0 \quad 0.9100 .0100 .0100 .0$ $1.0100 .0100 .0100 .0 \quad 0.6 \quad 0.6100 .0100 .0100 .0$ 
Mean Probebility of Exceedina Run Duration

\begin{tabular}{|c|c|c|c|c|c|c|c|c|c|}
\hline \multirow[b]{2}{*}{ Days } & & \multicolumn{8}{|c|}{ Northwest } \\
\hline & 1 & 2 & Wee & it her & $\begin{array}{c}\text { Pat tern } \\
5\end{array}$ & $\begin{array}{r}\text { TYoe } \\
6\end{array}$ & of Run & B & 4 \\
\hline $\begin{array}{l}1 \\
2 \\
3 \\
4 \\
5 \\
6 \\
7\end{array}$ & $\begin{array}{l}4.6 \\
0.0 \\
0.0 \\
0.0 \\
0.0 \\
0.0 \\
0.0\end{array}$ & $\begin{array}{r}14,3 \\
2,3 \\
0,3 \\
0,3 \\
0,1 \\
0,0 \\
0.0\end{array}$ & $\begin{array}{l}3.3 \\
0.2 \\
0.0 \\
0.0 \\
0.0 \\
0.0 \\
0.0\end{array}$ & $\begin{array}{r}37.0 \\
15.1 \\
6.7 \\
3.2 \\
1.5 \\
0.8 \\
0.9\end{array}$ & $\begin{array}{r}36.6 \\
15.6 \\
7.1 \\
3.3 \\
1.6 \\
0.9 \\
0.5\end{array}$ & $\begin{array}{l}6.3 \\
6.3 \\
0.0 \\
0.0 \\
0.0 \\
0.0 \\
0.0\end{array}$ & $\begin{array}{r}23.8 \\
7.3 \\
2.6 \\
1.3 \\
0.6 \\
0.3 \\
0.2\end{array}$ & $\begin{array}{r}14.8 \\
2.1 \\
0.4 \\
0.0 \\
0.0 \\
0.0 \\
0.0\end{array}$ & $\begin{array}{r}40.9 \\
21.3 \\
13.1 \\
8.8 \\
6.4 \\
4.8 \\
3.8\end{array}$ \\
\hline $\begin{array}{l}\text { Totel } \\
\text { rungas }\end{array}$ & $197 \mathrm{~A}$ & $572 n$ & 2653 & 14495 & 12689 & 16 & 8560 & 4325 & 16113 \\
\hline
\end{tabular}

Menn Probability of Exceodina Run Duration

$$
\text { North Central }
$$

Doys

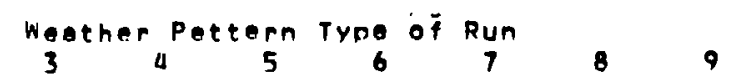

$\begin{array}{lrrrrrrrrr}1 & 8.3 & 12.2 & 3.6 & 34.9 & 36.0 & 38.7 & 22.0 & 24.0 & 20.5 \\ 2 & 0.8 & 1.5 & 0.0 & 11.3 & 12.6 & 18.8 & 5.5 & 0.9 & 4.9 \\ 3 & 0.1 & 0.1 & 0.0 & 4.1 & 4.7 & 7.2 & 1.6 & 1.4 & 1.3 \\ 4 & 0.0 & 0.1 & 0.0 & 1.9 & 2.0 & 3.4 & 0.4 & 0.4 & 0.2 \\ 5 & 0.0 & 0.0 & 0.0 & 0.5 & 0.8 & 1.4 & 0.2 & 0.2 & 0.1 \\ 6 & 0.0 & 0.0 & 0.0 & 0.2 & 0.0 & 1.0 & 0.1 & 0.0 & 0.0 \\ 7 & 0.0 & 0.0 & 0.0 & 0.1 & 0.2 & 0.5 & 0.0 & 0.0 & 0.0 \\ \text { Tot } 11 & & & & & & & & \\ \text { runs }= & 2625 & 5282 & 2327 & 11799 & 11804 & 1016 & 6144 & 3964 & 5241\end{array}$

Mean Probabilitr ó Exceedina Run Duration

Northenet

\begin{tabular}{|c|c|c|c|c|c|c|c|c|c|}
\hline ays & 1 & 2 & we & her & Pattern & $\begin{array}{r}\text { Type } \\
6\end{array}$ & of Run & 8 & \\
\hline $\begin{array}{l}1 \\
2 \\
3 \\
4 \\
5 \\
6 \\
7\end{array}$ & $\begin{array}{l}6.6 \\
0.5 \\
0.1 \\
0.0 \\
0.0 \\
0.0 \\
0.0\end{array}$ & $\begin{array}{r}12.1 \\
1.7 \\
0.0 \\
0.1 \\
0.0 \\
0,0 \\
0.0\end{array}$ & $\begin{array}{l}2.5 \\
0.0 \\
0.0 \\
0.0 \\
0.0 \\
0.0 \\
0.0\end{array}$ & $\begin{array}{r}30.0 \\
11.6 \\
4.2 \\
1.7 \\
0.6 \\
0.2 \\
0.1\end{array}$ & $\begin{array}{r}26.5 \\
7.1 \\
2.1 \\
0.5 \\
0.2 \\
0.0 \\
0.0\end{array}$ & $\begin{array}{r}31.7 \\
12.2 \\
5.2 \\
2.6 \\
1.2 \\
0.8 \\
0.5\end{array}$ & $\begin{array}{r}19.5 \\
3.5 \\
0.8 \\
0.2 \\
0.1 \\
0.0 \\
0.0\end{array}$ & $\begin{array}{r}31.3 \\
10.2 \\
4.2 \\
1.9 \\
1.1 \\
0.5 \\
0.2\end{array}$ & $\begin{array}{r}19 \\
5 \\
2\end{array}$ \\
\hline $\operatorname{un} 8=$ & 19 & 741 & 849 & 14793 & 10117 & 4221 & 5074 & 7420 & 609 \\
\hline
\end{tabular}




\begin{tabular}{|c|c|c|c|c|c|c|c|c|c|}
\hline \multirow[b]{2}{*}{ Doyo } & & \multicolumn{8}{|c|}{ Southwest } \\
\hline & 1 & 2 & 3 & her ${ }_{4}^{P}$ & $\begin{array}{c}5 \\
5\end{array}$ & $\begin{array}{c}\text { Troe } \\
6\end{array}$ & of Run & 8 & 9 \\
\hline $\begin{array}{l}1 \\
2 \\
3 \\
4 \\
5 \\
6 \\
7 \\
\text { Totol } 1\end{array}$ & $\begin{array}{l}3.5 \\
0.5 \\
0.0 \\
0.0 \\
0.0 \\
0.0 \\
0.0\end{array}$ & $\begin{array}{l}7.5 \\
0.6 \\
0,0 \\
0,0 \\
0.0 \\
0,0 \\
0.0\end{array}$ & $\begin{array}{l}0.2 \\
0.1 \\
0.0 \\
0.0 \\
0.0 \\
0.0 \\
0.0\end{array}$ & $\begin{array}{r}26.7 \\
9.0 \\
4.2 \\
1.7 \\
1.0 \\
0.6 \\
0.5\end{array}$ & $\begin{array}{r}39.6 \\
19.6 \\
6.8 \\
3.5 \\
1.8 \\
0.8 \\
0.5\end{array}$ & $\begin{array}{l}0.7 \\
4.3 \\
0.0 \\
0.0 \\
0.0 \\
0.0 \\
0.0\end{array}$ & $\begin{array}{r}13.1 \\
2.2 \\
0.2 \\
0.0 \\
0.0 \\
0.0 \\
0.0\end{array}$ & $\begin{array}{l}7.5 \\
1.0 \\
0.5 \\
0.0 \\
0.0 \\
0.0 \\
0.0\end{array}$ & $\begin{array}{l}64.0 \\
45.4 \\
34.7 \\
27.8 \\
22.6 \\
18.9 \\
16.7\end{array}$ \\
\hline une & 426 & 321 & 698 & 2798 & 3725 & 92 & 2009 & 386 & 6740 \\
\hline
\end{tabular}

Meon Probabilite of Exceedina Run Duretion

south centrol

\begin{tabular}{|c|c|c|c|c|c|c|c|c|c|}
\hline avs & & 2 & we & her ${ }_{4}$ & tern & Trne & of Run & A & \\
\hline $\begin{array}{l}1 \\
2 \\
3 \\
4 \\
5 \\
6 \\
7 \\
\text { Totol }\end{array}$ & $\begin{array}{l}6.0 \\
0.3 \\
0.0 \\
0.0 \\
0.0 \\
0.0 \\
0.0\end{array}$ & $\begin{array}{ll}5 & 0 \\
0.0 & 0 \\
0.0 & 0 \\
0.0 & 0 \\
0.0 & 0 \\
0.0 & 0 \\
0.0 & 0\end{array}$ & $\begin{array}{l}1.9 \\
0.0 \\
0.0 \\
0.0 \\
0.0 \\
0.0 \\
0.0\end{array}$ & $\begin{array}{r}32.7 \\
9.4 \\
3.2 \\
1.1 \\
0.4 \\
0.1 \\
0.0\end{array}$ & $\begin{array}{r}35.1 \\
11.9 \\
0.7 \\
1.6 \\
0.6 \\
0.3 \\
0.2\end{array}$ & $\begin{array}{r}54.1 \\
35.5 \\
24.6 \\
18.1 \\
13.8 \\
11.0 \\
1.8\end{array}$ & $\begin{array}{r}23.4 \\
5.6 \\
1.3 \\
0.4 \\
0.1 \\
0.1 \\
0.0\end{array}$ & $\begin{array}{l}7.9 \\
0.3 \\
0.0 \\
0.0 \\
0.0 \\
0.0 \\
0.0\end{array}$ & $\begin{array}{r}36.4 \\
16.1 \\
8.1 \\
4.8 \\
3.4 \\
2.3 \\
1.6\end{array}$ \\
\hline uns: & 1166 & 763 & 967 & 898 & 6790 & 6336 & 5057 & 366 & 5951 \\
\hline
\end{tabular}

Meen Prohobilitr of Exceading Run Duration

Davs

$$
\text { southoest }
$$$$
\text { Weother Pattern Troe of Run }
$$

$\begin{array}{llllllllll}1 & 4.7 & 4.7 & 3.1 & 41.7 & 23.0 & 60.9 & 24.5 & 12.7 & 26.4\end{array}$

$\begin{array}{llllllllll}1 & 0.7 & 4.7 & 3.1 & 41.0 & 23.0 & 60.9 & 24.5 & 12.7 & 26.9 \\ 2 & 0.4 & 0.2 & 0.2 & 14.8 & 0.0 & 51.2 & 7.3 & 1.7 & 10.1 \\ 3 & 0.0 & 0.0 & 0.0 & 6.0 & 1.5 & 29.3 & 1.6 & 1.0 & 4.3 \\ 4 & 0.0 & 0.0 & 0.0 & 2.2 & 0.6 & 21.8 & 0.5 & 0.2 & 2.0 \\ 5 & 0.0 & 0.0 & 0.0 & 0.9 & 0.2 & 17.0 & 0.2 & 0.0 & 1.1 \\ 6 & 0.0 & 0.0 & 0.0 & 0.5 & 0.1 & 13.2 & 0.1 & 0.0 & 0.5 \\ 7 & 0.0 & 0.0 & 0.0 & 0.2 & 0.1 & 10.8 & 0.0 & 0.0 & 0.3 \\ \text { Total } & & & & & & & \\ \text { rung= } & 1354 & 089 & 1201 & 6051 & 2378 & 7867 & 4429 & 518 & 4293\end{array}$


APPENDIX E 



\section{PERCENTAGE PROBABILITY OF WEATHER PATTERN ALTERNATION}

The data listings in this appendix present the percentage probability that runs of one weather pattern type are terminated by alternation to another given type. That is, one could find the probability that a run of weather pattern Type 1 is ended by alternation to weather pattern Type 3. Nine sets of tables are presented, one for each current run weather pattern type. Summaries for the six regions are presented after the tables.

All of the tables are presented by latitude band across the region. The 10 bands are presented from north to south. Seventeen columns of data are presented in each band. The first column denotes the new weather pattern type. The other 16 columns are headed by the longitude of the grid point for which the percentage probability that the new weather pattern terminate the current run applies.

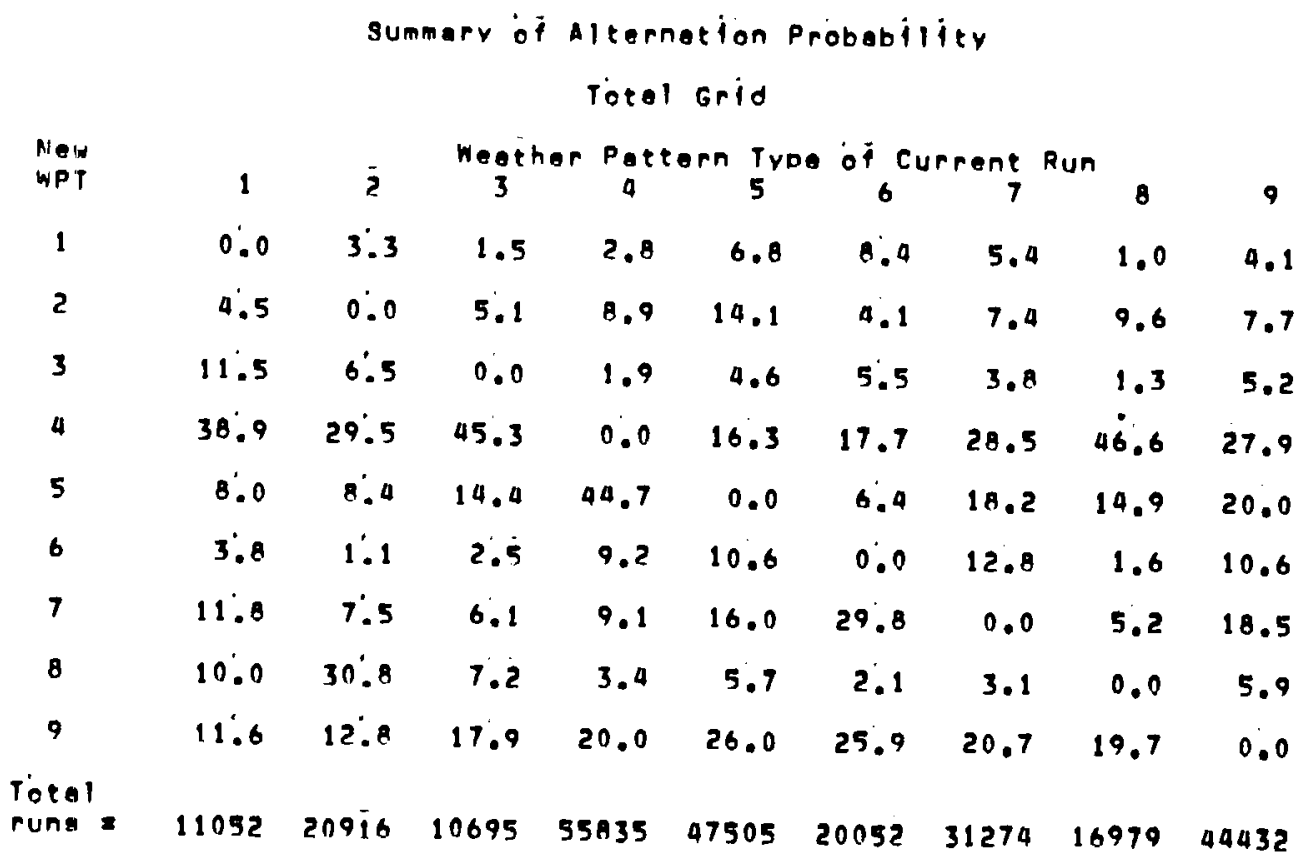


51 Dagraes North Latitude

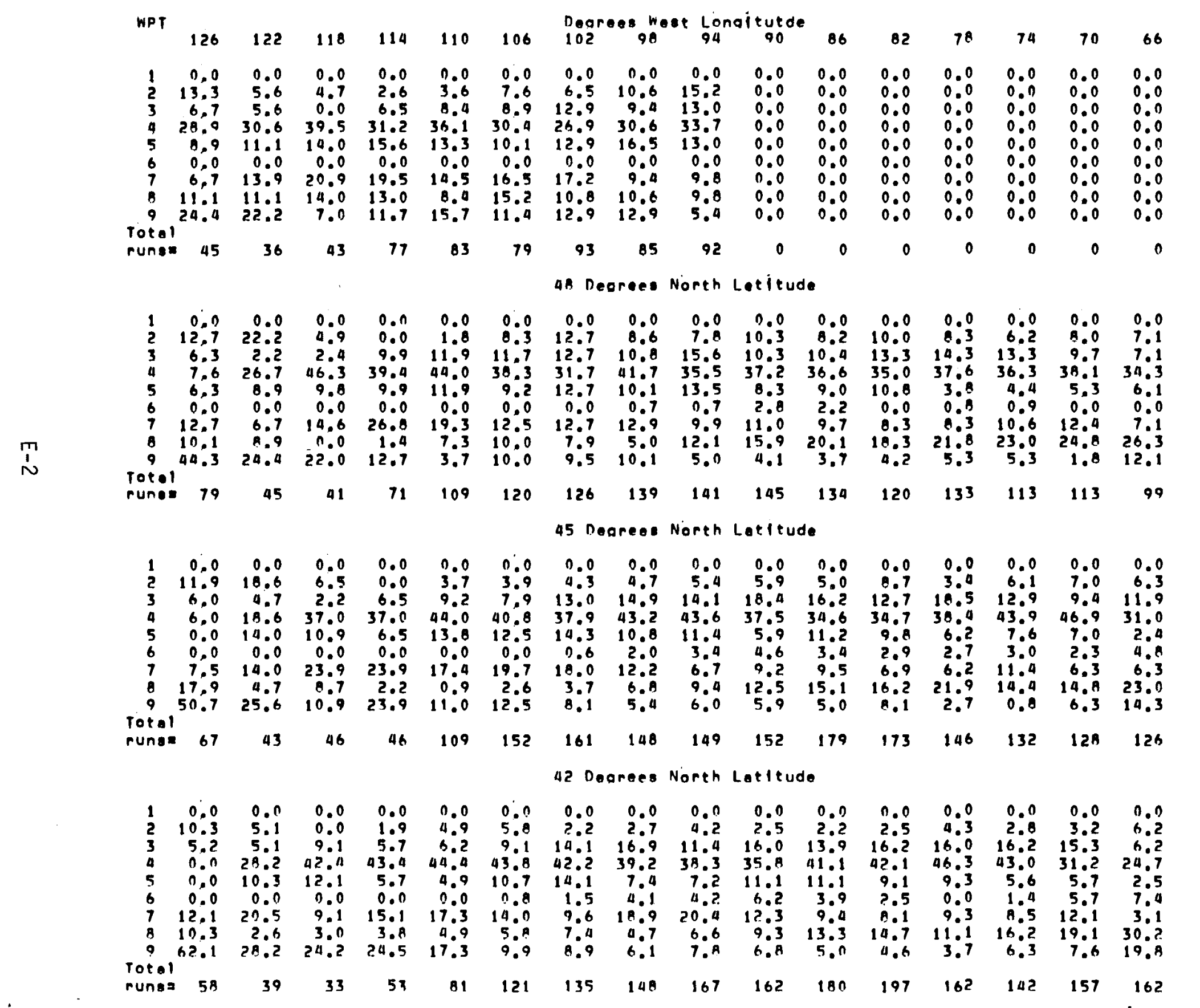


Percentage of Runs of WPT 1 thet Change to WPT shown

39 Dagrees Nopth Lotitude

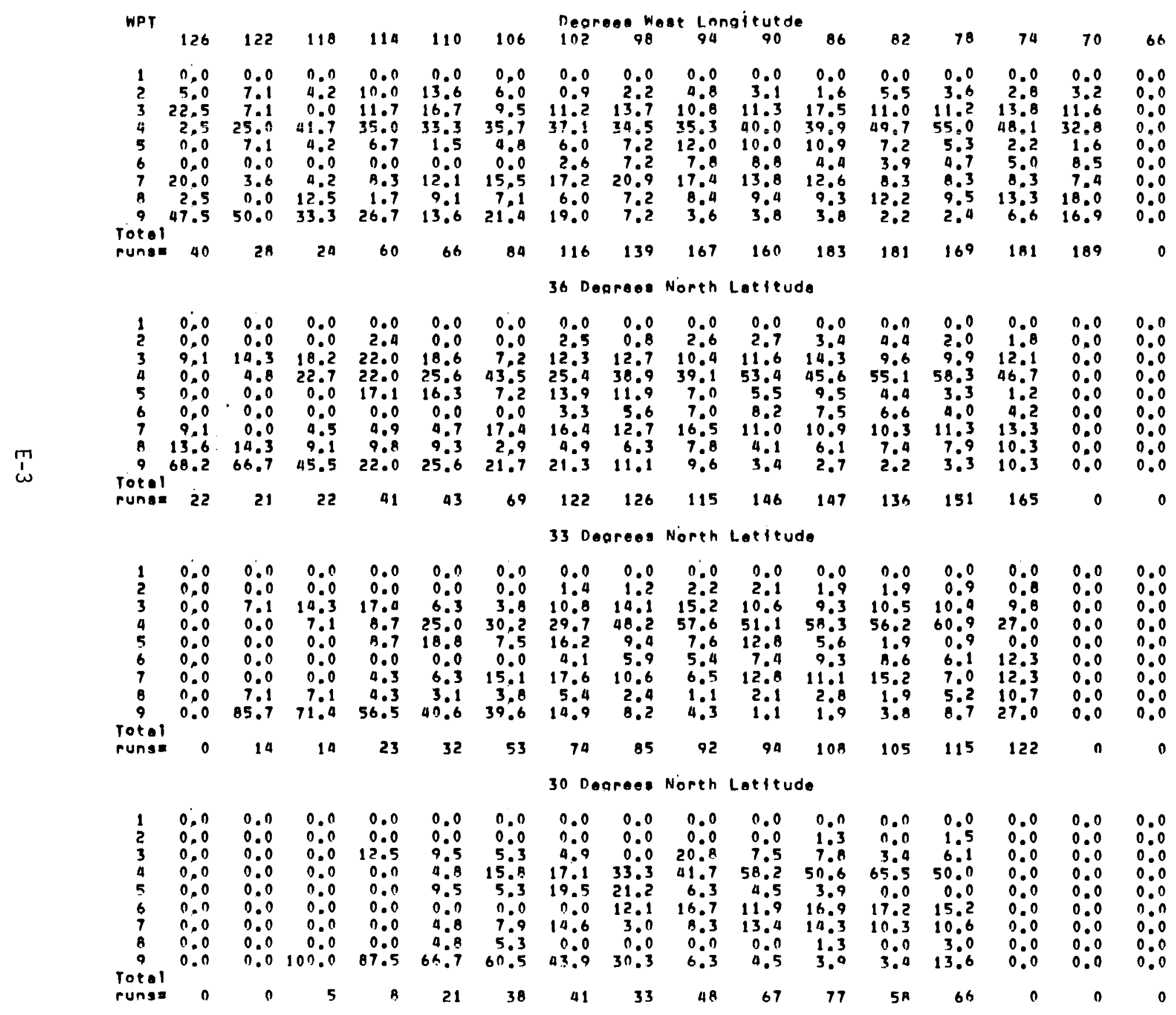


Percentage of Runa of WPT 1 thot Change to WPT shown

27 Degrees North Latitude

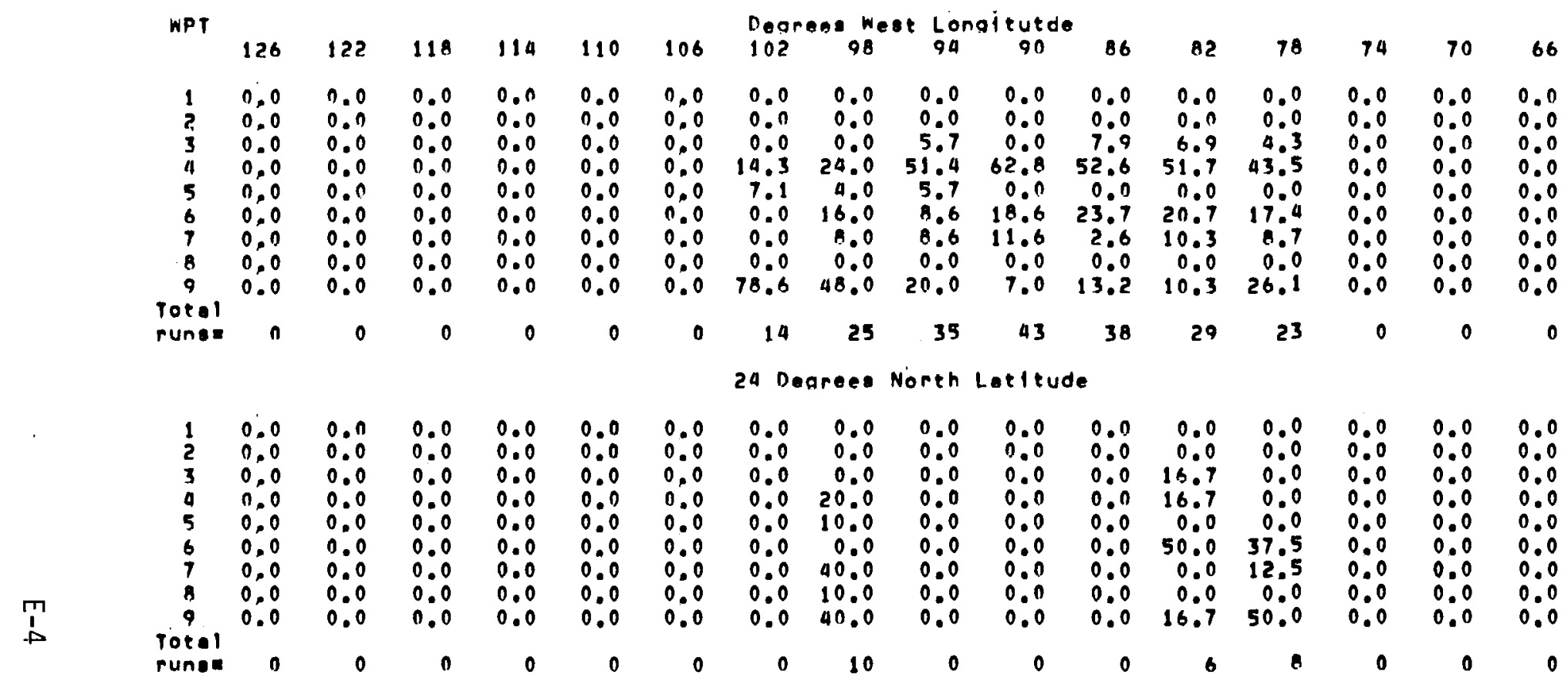


Percentage of Rune of WPT 2 thet Change to WPT shown

51 Deprees North Lotltude

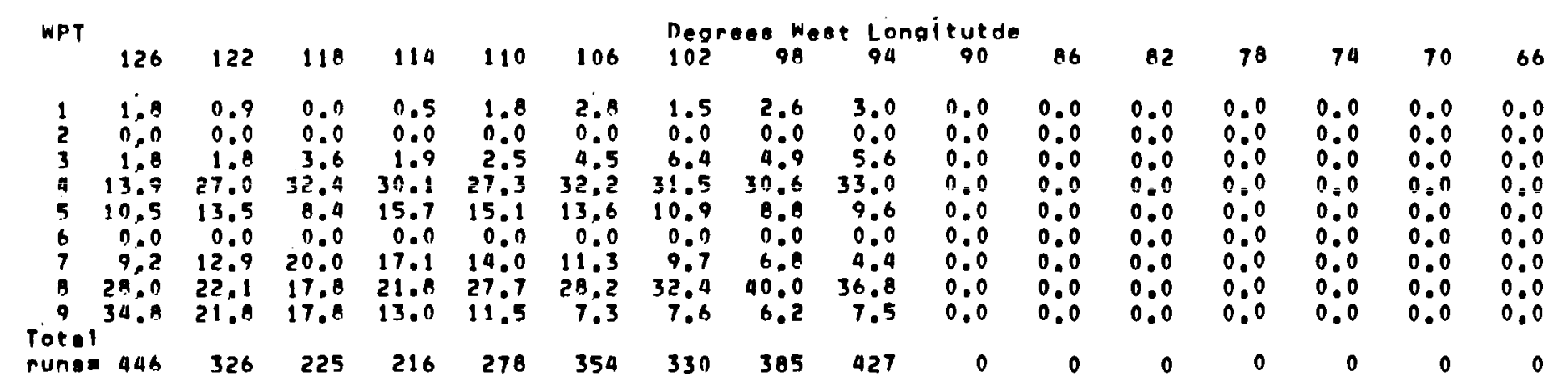

48 Degrees North Lotitude

\begin{tabular}{|c|c|c|c|c|c|c|c|c|c|c|c|c|c|c|c|c|}
\hline $\begin{array}{l}1 \\
2 \\
3 \\
9 \\
5 \\
6 \\
7 \\
8 \\
9\end{array}$ & $\begin{array}{r}3.0 \\
.0 \\
.9 \\
.7 \\
.3 \\
.0 \\
.4 \\
.0 \\
41.6\end{array}$ & $\begin{array}{r}2.6 \\
0.0 \\
3.2 \\
24.2 \\
13.2 \\
0.0 \\
9.0 \\
23.9 \\
23.9\end{array}$ & $\begin{array}{r}0.4 \\
0.0 \\
3.9 \\
32.9 \\
8.3 \\
0.0 \\
17.1 \\
19.3 \\
18.0\end{array}$ & $\begin{array}{r}0.0 \\
0.0 \\
2.2 \\
37.0 \\
9.2 \\
0.0 \\
22.3 \\
17.9 \\
11.4\end{array}$ & $\begin{array}{r}0.5 \\
0.0 \\
5.4 \\
36.5 \\
9.5 \\
0.0 \\
16.7 \\
20.7 \\
10.8\end{array}$ & $\begin{array}{r}2.2 \\
0.0 \\
5.1 \\
36.4 \\
11.0 \\
0.0 \\
14.3 \\
23.2 \\
7.7\end{array}$ & $\begin{array}{r}1.6 \\
0.0 \\
6.2 \\
32.5 \\
10.4 \\
0.0 \\
12.3 \\
28.6 \\
8.4\end{array}$ & $\begin{array}{r}3.5 \\
0.0 \\
5.5 \\
33.5 \\
9.0 \\
0.0 \\
7.9 \\
33.5 \\
7.0\end{array}$ & $\begin{array}{r}3.4 \\
0.0 \\
7.0 \\
29.8 \\
11.2 \\
0.3 \\
5.5 \\
34.5 \\
8.4\end{array}$ & $\begin{array}{r}2.2 \\
0.0 \\
5.9 \\
31.8 \\
11.7 \\
0.5 \\
3.7 \\
37.7 \\
6.6\end{array}$ & $\begin{array}{r}3.7 \\
0.0 \\
5.1 \\
30.9 \\
9.8 \\
0.7 \\
3.5 \\
40.4 \\
7.0\end{array}$ & $\begin{array}{r}2.4 \\
0.0 \\
3.4 \\
31.0 \\
9.1 \\
0.7 \\
5.5 \\
36.5 \\
8.4\end{array}$ & $\begin{array}{r}3.1 \\
0.0 \\
5.1 \\
33.9 \\
7.8 \\
0.9 \\
8.0 \\
39.0 \\
6.2\end{array}$ & $\begin{array}{r}2.9 \\
0.0 \\
4.5 \\
32.7 \\
7.2 \\
0.7 \\
2.7 \\
42.8\end{array}$ & $\begin{array}{r}0.5 \\
3.4 \\
48.4 \\
6.6\end{array}$ & $\begin{array}{r}2.2 \\
0.0 \\
4.3 \\
22.7 \\
3.2 \\
1.9 \\
3.2 \\
50.0 \\
12.3\end{array}$ \\
\hline & & 1 & 220 & 1 & 28 & 27 & 308 & 343 & $3 t$ & 409 & 431 & 452 & 449 & 44 & 140 & 162 \\
\hline
\end{tabular}

45 Degrees North Latteve

\begin{tabular}{|c|c|c|c|c|c|c|c|c|c|c|c|c|c|c|c|c|}
\hline $\begin{array}{l}1 \\
2 \\
3 \\
4 \\
5 \\
6 \\
7 \\
6 \\
9\end{array}$ & $\begin{array}{r}3.0 \\
0.0 \\
4.0 \\
1.0 \\
1.5 \\
0.0 \\
5.0 \\
34.2 \\
51.3\end{array}$ & $\begin{array}{r}1.9 \\
0.0 \\
5.2 \\
20.7 \\
11.7 \\
0.0 \\
8.5 \\
2 ? .1 \\
30.0\end{array}$ & $\begin{array}{r}2.0 \\
0.0 \\
2.4 \\
28.2 \\
8.8 \\
0.0 \\
9.8 \\
21.8 \\
27.6\end{array}$ & $\begin{array}{r}0.7 \\
0.0 \\
1.4 \\
30.1 \\
7.5 \\
0.0 \\
15.8 \\
17.1 \\
27.4\end{array}$ & $\begin{array}{r}0.7 \\
0.0 \\
5.6 \\
38.7 \\
9.2 \\
0.0 \\
14.8 \\
19.7 \\
11.3\end{array}$ & $\begin{array}{r}2.7 \\
0.0 \\
4.8 \\
35.1 \\
10.1 \\
0.0 \\
14.9 \\
23.9 \\
8.5\end{array}$ & $\begin{array}{r}3.4 \\
0.0 \\
7.6 \\
34.5 \\
10.1 \\
0.0 \\
13.4 \\
24.4 \\
6.7\end{array}$ & $\begin{array}{r}4.5 \\
0.0 \\
9.6 \\
32.3 \\
8.9 \\
0.0 \\
9.3 \\
25.4 \\
10.0\end{array}$ & $\begin{array}{r}4.8 \\
0.0 \\
8.6 \\
27.9 \\
12.4 \\
0.3 \\
7.6 \\
29.5 \\
8.9\end{array}$ & $\begin{array}{r}3.9 \\
0.0 \\
4.5 \\
34.8 \\
10.4 \\
1.2 \\
4.8 \\
35.4 \\
5.1\end{array}$ & $\begin{array}{r}3.9 \\
0.0 \\
8.6 \\
23.0 \\
7.8 \\
0.3 \\
4.7 \\
44.9 \\
6.9\end{array}$ & $\begin{array}{r}6.3 \\
0.0 \\
5.5 \\
27.0 \\
7.6 \\
1.8 \\
6.5 \\
40.6 \\
4.8\end{array}$ & $\begin{array}{r}9.1 \\
0.0 \\
6.6 \\
29.7 \\
8.5 \\
1.2 \\
5.1 \\
38.7 \\
6.1\end{array}$ & $\begin{array}{r}2.5 \\
0.0 \\
6.2 \\
30.4 \\
7.2 \\
0.7 \\
4.7 \\
42.6 \\
5.5\end{array}$ & $\begin{array}{r}4.2 \\
0.0 \\
7.3 \\
30.1 \\
3.4 \\
1.5 \\
4.9 \\
40.8 \\
7.8\end{array}$ & $\begin{array}{r}3.1 \\
0.0 \\
4.4 \\
20.5 \\
3.1 \\
2.1 \\
4.9 \\
48.1 \\
13.8\end{array}$ \\
\hline $\begin{array}{l}\text { Totol } \\
\text { runas }\end{array}$ & 199 & 213 & 170 & 146 & 142 & 188 & 231 & 291 & 315 & 336 & 361 & 397 & 411 & 401 & 409 & 385 \\
\hline & & & & & & & $42 \mathrm{De}$ & arees & North & Lotite & & & & & & \\
\hline $\begin{array}{c}1 \\
2 \\
3 \\
4 \\
5 \\
6 \\
7 \\
6 \\
9 \\
\text { Total }\end{array}$ & $\begin{array}{r}4.4 \\
0.0 \\
5.1 \\
0.0 \\
0.0 \\
0.0 \\
3.7 \\
25.7 \\
61.0\end{array}$ & $\begin{array}{r}1.6 \\
0.0 \\
5.5 \\
13.3 \\
5.5 \\
0.0 \\
10.9 \\
24.2 \\
39.1\end{array}$ & $\begin{array}{r}1.8 \\
0.0 \\
2.7 \\
29.7 \\
6.3 \\
0.0 \\
6.3 \\
26.1 \\
27.0\end{array}$ & $\begin{array}{r}0.0 \\
0.0 \\
1.6 \\
30.6 \\
0.1 \\
0.0 \\
9.7 \\
23.4 \\
26.6\end{array}$ & $\begin{array}{r}2.4 \\
0.0 \\
4.0 \\
32.3 \\
8.9 \\
0.0 \\
20.2 \\
16.1 \\
16.1\end{array}$ & $\begin{array}{r}0.0 \\
0.0 \\
4.2 \\
36.8 \\
15.3 \\
0.7 \\
11.1 \\
18.9 \\
13.2\end{array}$ & $\begin{array}{r}1.7 \\
0.0 \\
3.9 \\
34.9 \\
15.7 \\
0.0 \\
9.4 \\
21.3 \\
14.0\end{array}$ & $\begin{array}{r}3.6 \\
0.0 \\
6.2 \\
31.3 \\
10.8 \\
0.0 \\
10.3 \\
28.7 \\
9.2\end{array}$ & $\begin{array}{r}1.9 \\
0.0 \\
9.8 \\
35.6 \\
12.4 \\
0.9 \\
4.9 \\
27.6 \\
4.4\end{array}$ & $\begin{array}{r}5.8 \\
0.0 \\
7.0 \\
32.3 \\
12.5 \\
1.2 \\
6.2 \\
30.7 \\
4.3\end{array}$ & $\begin{array}{r}4.0 \\
0.0 \\
9.0 \\
29.1 \\
10.8 \\
2.2 \\
5.4 \\
35.6 \\
4.0\end{array}$ & $\begin{array}{r}6.3 \\
0.0 \\
7.8 \\
30.1 \\
9.2 \\
0.9 \\
6.9 \\
36.1 \\
3.1\end{array}$ & $\begin{array}{r}3.9 \\
0.0 \\
7.7 \\
32.4 \\
6.3 \\
1.8 \\
5.1 \\
37.5 \\
5.4\end{array}$ & $\begin{array}{r}4.7 \\
0.0 \\
8.7 \\
37.0 \\
5.0 \\
1.2 \\
5.2 \\
32.9 \\
5.2\end{array}$ & $\begin{array}{r}4.0 \\
0.0 \\
9.6 \\
26.6 \\
2.3 \\
2.3 \\
4.7 \\
40.5 \\
10.0\end{array}$ & $\begin{array}{r}5.6 \\
0.0 \\
4.8 \\
17.9 \\
1.8 \\
3.6 \\
6.8 \\
44.6 \\
15.5\end{array}$ \\
\hline runse & - 136 & $12 A$ & 111 & 124 & 124 & 144 & 178 & 195 & 225 & 257 & $27 \mathrm{~A}$ & 319 & 336 & 343 & 301 & 251 \\
\hline
\end{tabular}


Percentage of Rung of WPT 2 that Change to WPT ghown

39 Degraes North Latltude

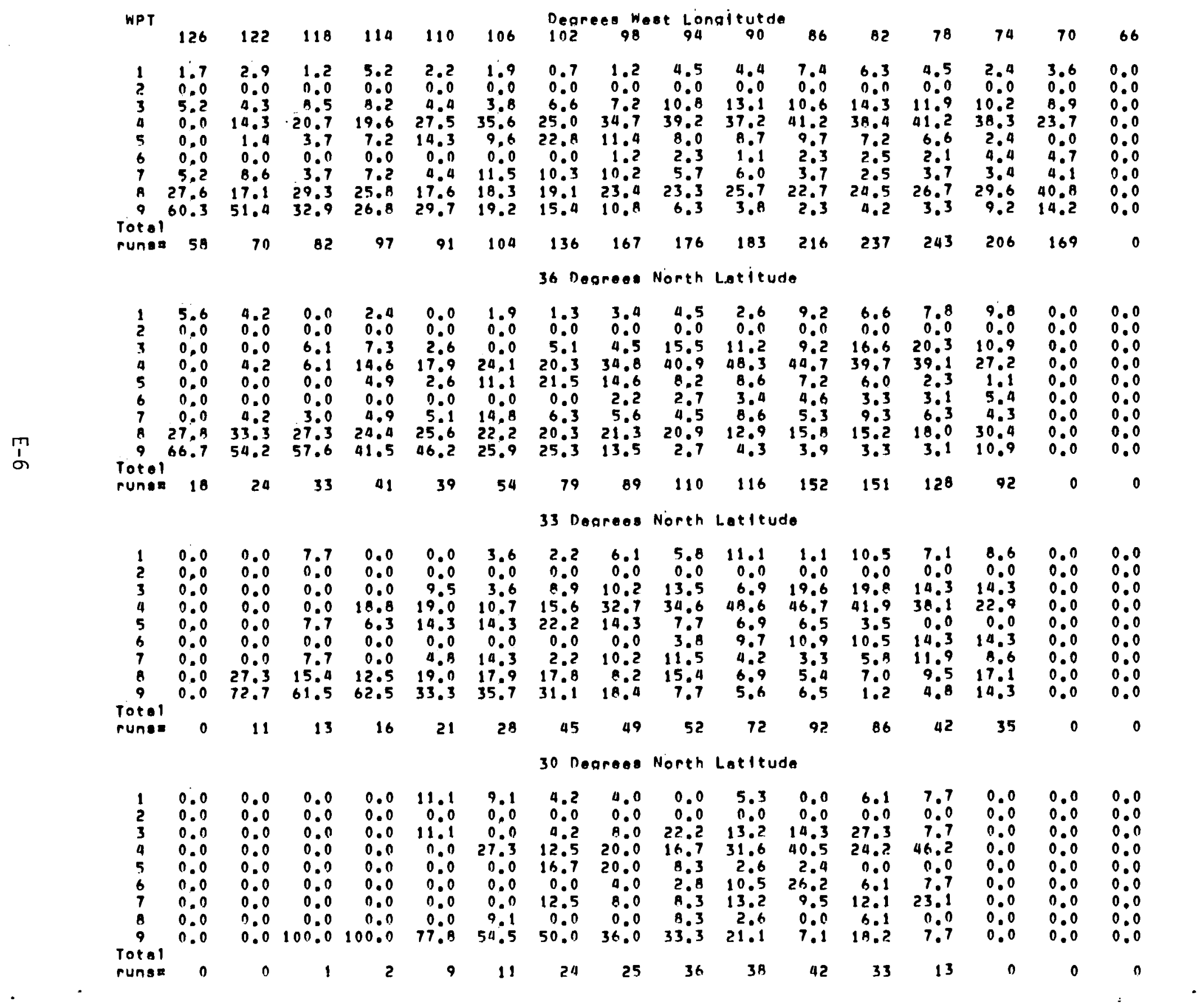


Percentage of Runs of WPT 2 that Change to WPT shown

27 Degrees North Latlude

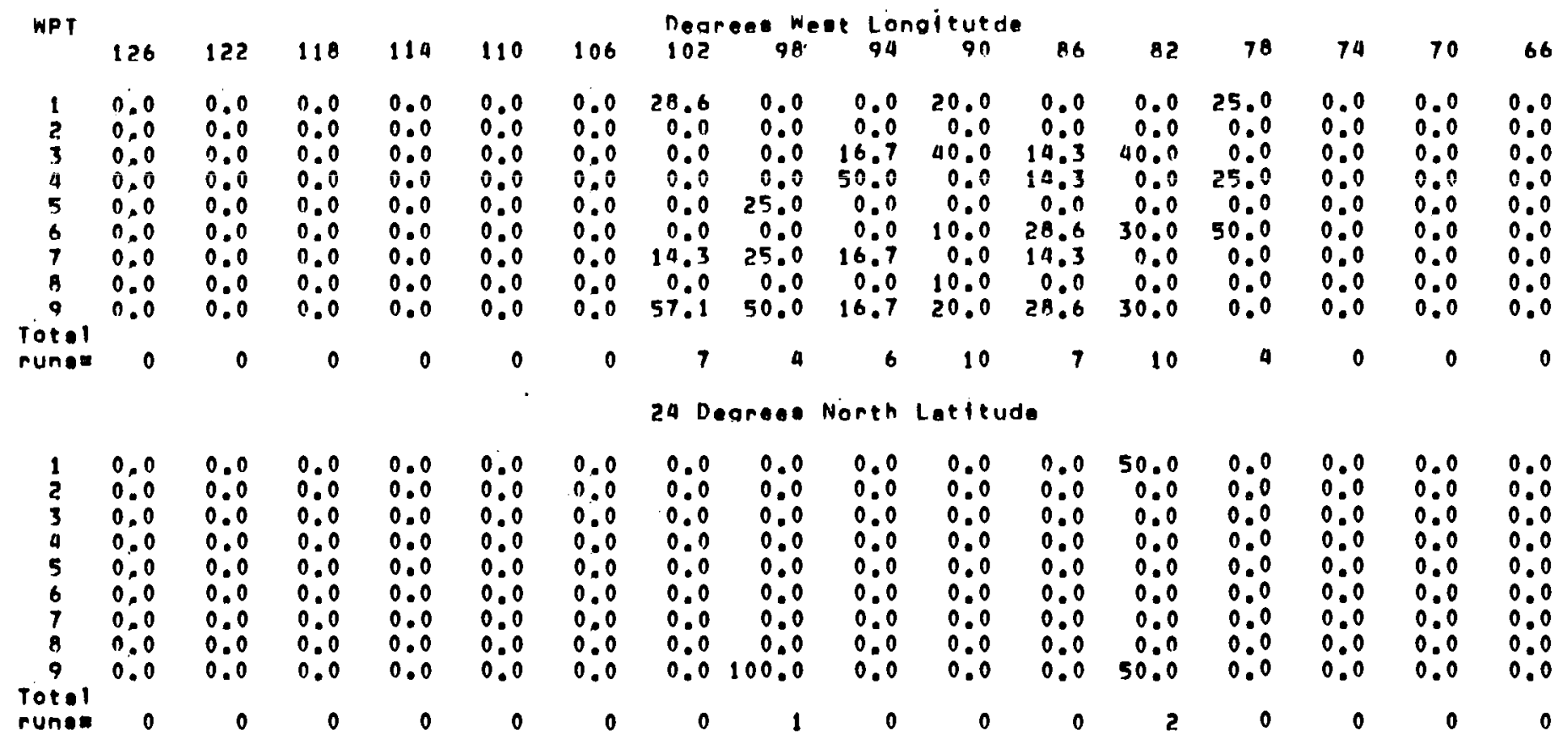


Percentege of Runs of WPT 3 that Change to WPT Shown

51 Degree North Lattide

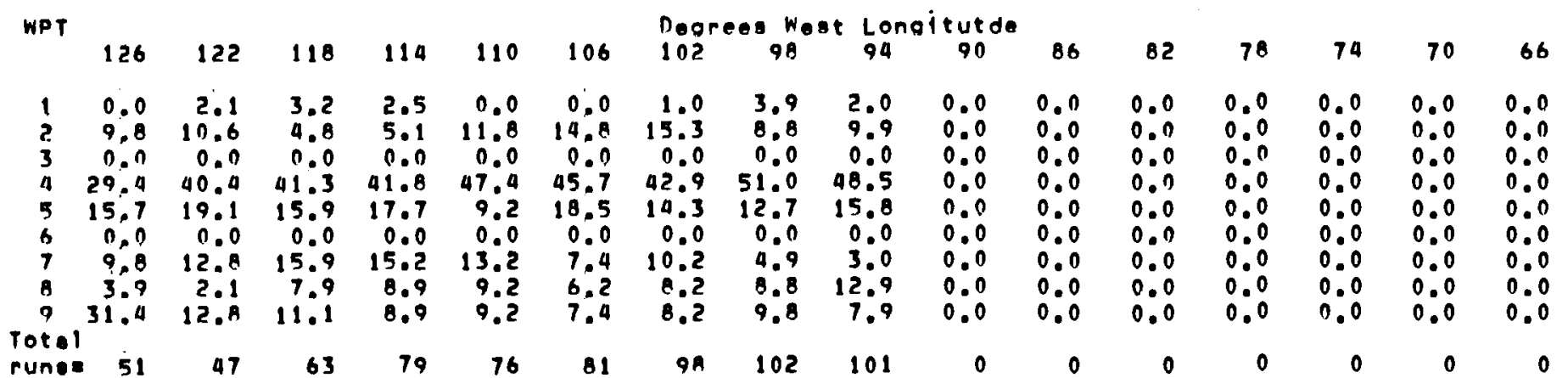

48 Deqrees North Latitude

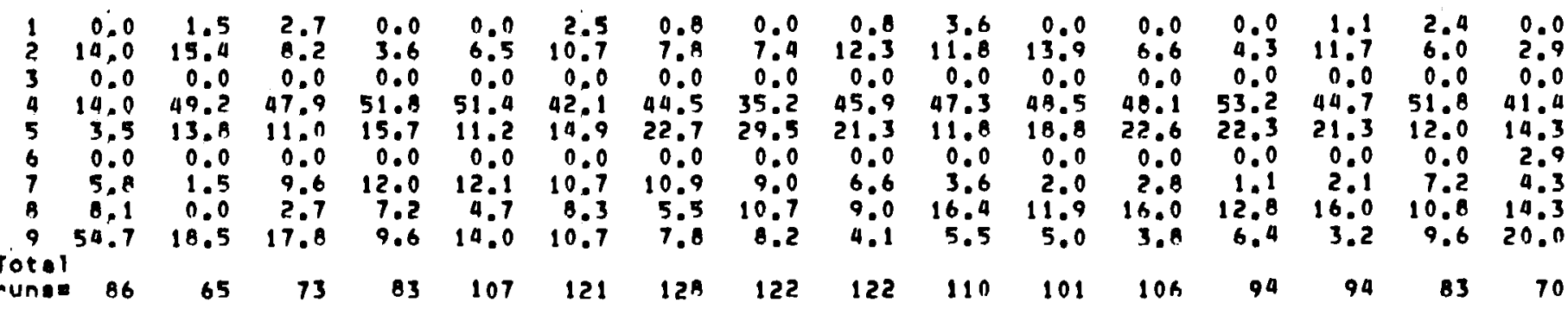

45 Degreese North Latitude

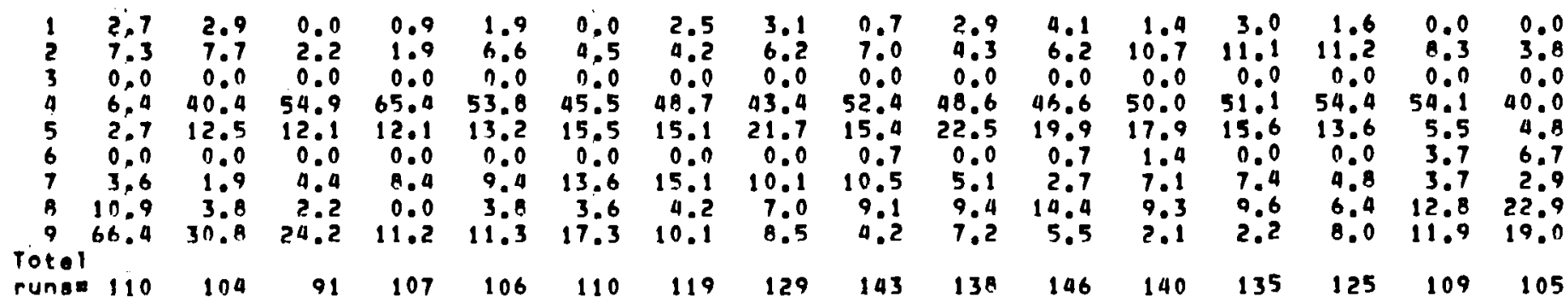

42 Degrees North Letftude

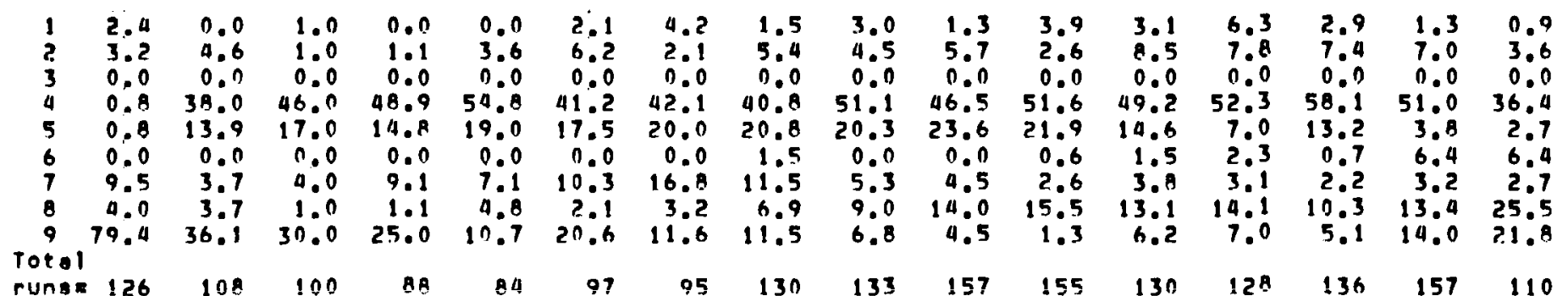


Parcentage of Runs of WPT 3 that Change to WPT shown

39 Dogreos North Latltude

\begin{tabular}{|c|c|c|c|c|c|c|c|c|c|c|c|c|c|c|c|c|}
\hline WPT & 126 & 122 & 118 & 114 & 110 & 106 & $\begin{array}{l}\text { Dear } \\
102\end{array}$ & 98 & It $\begin{array}{l}\text { Lon } \\
94\end{array}$ & $\begin{array}{l}\text { tut de } \\
90\end{array}$ & 86 & 82 & 78 & 74 & 70 & 66 \\
\hline $\begin{array}{c}1 \\
2 \\
3 \\
4 \\
5 \\
6 \\
7 \\
6 \\
9 \\
\text { ot } 1 \\
\text { Una } 1\end{array}$ & $\begin{array}{r}3.0 \\
2.0 \\
0.0 \\
0.0 \\
0.0 \\
0.0 \\
2.0 \\
11.0 \\
81.8 \\
99\end{array}$ & $\begin{array}{r}0.0 \\
3.0 \\
0.0 \\
23.2 \\
14.1 \\
0.0 \\
0.0 \\
0.0 \\
59.6\end{array}$ & $\begin{array}{r}1.3 \\
5.2 \\
0.0 \\
36.4 \\
18.2 \\
0.0 \\
2.6 \\
0.0 \\
36.4\end{array}$ & $\begin{array}{r}1.4 \\
4.1 \\
0.0 \\
35.1 \\
28.4 \\
0.0 \\
6.0 \\
2.7 \\
21.6\end{array}$ & $\begin{array}{r}1.4 \\
4.3 \\
0.0 \\
50.0 \\
11.4 \\
0.0 \\
5.7 \\
2.9 \\
24.3 \\
70\end{array}$ & $\begin{array}{r}1.4 \\
2.8 \\
0.9 \\
49.3 \\
16.9 \\
0.0 \\
8.5 \\
2.8 \\
18.3 \\
71\end{array}$ & $\begin{array}{r}0.0 \\
2.0 \\
0.0 \\
43.0 \\
21.0 \\
0.0 \\
15.0 \\
5.0 \\
14.0 \\
100\end{array}$ & $\begin{array}{r}2.7 \\
4.5 \\
0.0 \\
40.2 \\
17.9 \\
0.9 \\
16.1 \\
5.9 \\
12.5 \\
112\end{array}$ & $\begin{array}{r}2.4 \\
1.6 \\
0.0 \\
50.0 \\
19.4 \\
3.2 \\
8.9 \\
6.5 \\
8.1 \\
124\end{array}$ & $\begin{array}{r}0.0 \\
3.0 \\
0.0 \\
50.3 \\
19.5 \\
3.7 \\
6.1 \\
8.5 \\
4.9 \\
164\end{array}$ & $\begin{array}{r}3.4 \\
6.1 \\
0.0 \\
53.7 \\
19.3 \\
5.4 \\
2.7 \\
11.6 \\
2.7 \\
147\end{array}$ & $\begin{array}{r}0.7 \\
5.9 \\
0.0 \\
66.2 \\
12.5 \\
2.2 \\
2.9 \\
7.9 \\
2.2 \\
136\end{array}$ & $\begin{array}{r}0.7 \\
6.4 \\
0.0 \\
59.6 \\
10.0 \\
2.1 \\
6.4 \\
9.9 \\
4.3 \\
141\end{array}$ & $\begin{array}{r}2.7 \\
5.4 \\
0.0 \\
57.1 \\
6.1 \\
6.8 \\
3.4 \\
5.4 \\
12.9 \\
147\end{array}$ & $\begin{array}{r}1.3 \\
3.9 \\
0.0 \\
40.3 \\
2.0 \\
9.1 \\
3.2 \\
14.9 \\
24.7 \\
154\end{array}$ & $\begin{array}{l}0.0 \\
0.0 \\
0.00 \\
0.0 \\
0.00 \\
0.0 \\
0.0 \\
0.0 \\
0.0\end{array}$ \\
\hline & & & & & & & $6 \mathrm{De}$ & 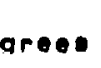 & orth & at teue & & & & & & \\
\hline $\begin{array}{c}1 \\
2 \\
3 \\
4 \\
5 \\
6 \\
7 \\
3 \\
9 \\
\text { otal } \\
\text { Unon }\end{array}$ & $\begin{array}{r}1.7 \\
1.7 \\
0.0 \\
0.0 \\
0.0 \\
0.0 \\
1.7 \\
6.9 \\
87.9 \\
58\end{array}$ & $\begin{array}{r}0.0 \\
0.0 \\
0.0 \\
5.7 \\
5.7 \\
0.0 \\
0.0 \\
5.7 \\
83.0\end{array}$ & $\begin{array}{r}0.0 \\
0.0 \\
0.0 \\
27.8 \\
12.7 \\
0.0 \\
0.0 \\
3.8 \\
55.7 \\
79\end{array}$ & $\begin{array}{r}0.0 \\
3.0 \\
0.0 \\
30.3 \\
21.2 \\
0.0 \\
1.5 \\
3.0 \\
40.9 \\
66\end{array}$ & $\begin{array}{r}0.0 \\
4.9 \\
0.0 \\
31.7 \\
22.0 \\
0.0 \\
4.9 \\
0.0 \\
36.6 \\
41\end{array}$ & $\begin{array}{r}0.0 \\
2.8 \\
0.0 \\
46.5 \\
11.3 \\
0.0 \\
15.5 \\
1.4 \\
22.5 \\
71\end{array}$ & $\begin{array}{r}2.3 \\
0.0 \\
0.0 \\
32.2 \\
28.7 \\
0.0 \\
10.3 \\
1.1 \\
25.3 \\
87\end{array}$ & $\begin{array}{r}2.6 \\
2.6 \\
0.0 \\
40.7 \\
30.3 \\
2.6 \\
3.9 \\
1.3 \\
11.8 \\
76\end{array}$ & $\begin{array}{r}0.0 \\
2.7 \\
0.0 \\
49.1 \\
31.8 \\
0.9 \\
5.5 \\
6.4 \\
3.6 \\
110\end{array}$ & $\begin{array}{r}0.0 \\
2.9 \\
0.0 \\
56.2 \\
21.0 \\
2.9 \\
5.7 \\
8.6 \\
2.9 \\
105\end{array}$ & $\begin{array}{r}1.7 \\
4.1 \\
0.0 \\
68.6 \\
16.5 \\
2.5 \\
0.0 \\
4.1 \\
2.5 \\
121\end{array}$ & $\begin{array}{r}2.3 \\
2.3 \\
0.0 \\
68.5 \\
12.3 \\
3.1 \\
3.1 \\
3.8 \\
4.6 \\
130\end{array}$ & $\begin{array}{r}2.2 \\
1.5 \\
0.0 \\
53.7 \\
4.5 \\
7.5 \\
4.5 \\
9.7 \\
16.4 \\
134\end{array}$ & $\begin{array}{r}2.7 \\
2.7 \\
0.0 \\
51.4 \\
4.1 \\
9.6 \\
4.1 \\
0.2 \\
17.1 \\
146\end{array}$ & $\begin{array}{ll}0.0 & 0 \\
0.0 & 0 \\
0.0 & 0 \\
0.0 & 0 \\
0.0 & 0 \\
0.0 & 0 \\
0.0 & 0 \\
0.0 & 0 \\
0.0 & 0\end{array}$ & $\begin{array}{l}0.0 \\
0.0 \\
0.0 \\
0.0 \\
0.0 \\
0.0 \\
0.0 \\
0.0 \\
0.0\end{array}$ \\
\hline & & & & & & & 330 & ee & rth & t1t & & & & & & \\
\hline $\begin{array}{l}1 \\
2 \\
3 \\
4 \\
5 \\
6 \\
7 \\
8 \\
9 \\
\text { otal } \\
\text { Una a }\end{array}$ & $\begin{array}{l}0.0 \\
0.0 \\
0.0 \\
0.0 \\
0.0 \\
0.0 \\
0.0 \\
0.0 \\
0.0 \\
0.0\end{array}$ & $\begin{array}{r}2.0 \\
0.0 \\
0.0 \\
0.0 \\
0.0 \\
0.0 \\
0.0 \\
9.5 \\
88.1 \\
42\end{array}$ & $\begin{array}{r}0.0 \\
0.0 \\
0.0 \\
13.6 \\
6.8 \\
0.0 \\
0.0 \\
0.0 \\
79.5 \\
44\end{array}$ & $\begin{array}{r}2.0 \\
0.0 \\
0.0 \\
23.5 \\
15.7 \\
0.0 \\
0.0 \\
2.0 \\
56.9 \\
51\end{array}$ & $\begin{array}{r}0.0 \\
0.0 \\
0.0 \\
18.8 \\
18.8 \\
0.0 \\
0.0 \\
6.3 \\
56.3 \\
32\end{array}$ & $\begin{array}{rl}0 . & 0 \\
2 & 0 \\
0.0 \\
32.0 \\
6.0 \\
0.0 \\
14.0 \\
2.00 \\
44.0 \\
50\end{array}$ & $\begin{array}{r}2.7 \\
0.0 \\
0.0 \\
26.7 \\
29.3 \\
1.3 \\
10.7 \\
0.0 \\
29.3 \\
75\end{array}$ & $\begin{array}{r}0.0 \\
3.4 \\
0.0 \\
40.1 \\
25.4 \\
1.7 \\
6.5 \\
1.7 \\
15.3 \\
59\end{array}$ & $\begin{array}{r}0.0 \\
1.3 \\
0.0 \\
52.6 \\
29.5 \\
3.8 \\
5.1 \\
2.6 \\
5.1 \\
78\end{array}$ & $\begin{array}{r}2.7 \\
1.4 \\
0.0 \\
64.4 \\
20.5 \\
4.1 \\
4.1 \\
1.4 \\
1.4 \\
73\end{array}$ & $\begin{array}{r}1.1 \\
2.2 \\
0.0 \\
73.3 \\
11.1 \\
3.3 \\
5.6 \\
1.1 \\
2.2 \\
90\end{array}$ & $\begin{array}{r}1.9 \\
1.9 \\
0.0 \\
70.9 \\
4.9 \\
5.9 \\
1.9 \\
2.9 \\
6.8 \\
103\end{array}$ & $\begin{array}{r}1.9 \\
2.9 \\
0.0 \\
45.7 \\
4.8 \\
6.7 \\
5.7 \\
5.7 \\
26.7 \\
105\end{array}$ & $\begin{array}{r}1.0 \\
0.0 \\
0.0 \\
47.1 \\
1.9 \\
11.5 \\
3.8 \\
6.7 \\
27.9 \\
104\end{array}$ & $\begin{array}{ll}0 & 0 \\
0 & 0 \\
0.0 & 0 \\
0.0 & 0 \\
0.0 & 0 \\
0.0 & 0 \\
0.0 & 0 \\
0.0 & 0 \\
0.0 & 0\end{array}$ & $\begin{array}{l}0.0 \\
0.00 \\
0.0 \\
0.0 \\
0.0 \\
0.0 \\
0.0 \\
0.0 \\
0.0\end{array}$ \\
\hline & & & & & & & 300 & rees & North & at I tue & & & & & & \\
\hline $\begin{array}{l}1 \\
2 \\
3 \\
4 \\
5 \\
6 \\
7 \\
2 \\
9\end{array}$ & $\begin{array}{l}0.0 \\
0.0 \\
0.0 \\
0.0 \\
0.0 \\
0.0 \\
0.0 \\
0.0 \\
0.0\end{array}$ & $\begin{array}{l}0.0 \\
0.0 \\
0.0 \\
0.0 \\
0.0 \\
0.0 \\
0.0 \\
0.0 \\
0.0\end{array}$ & $\begin{array}{r}0.0 \\
0.0 \\
0.0 \\
0.0 \\
0.0 \\
0.0 \\
0.0 \\
0.0 \\
100.0\end{array}$ & $\begin{array}{r}0.0 \\
0.0 \\
0.0 \\
12.1 \\
6.1 \\
0.0 \\
0.0 \\
0.0 \\
81.8\end{array}$ & $\begin{array}{r}0.0 \\
0.0 \\
0.0 \\
0.0 \\
0.7 \\
0.0 \\
4.3 \\
0.0 \\
67.0\end{array}$ & $\begin{array}{r}0.0 \\
0.0 \\
0.0 \\
15.4 \\
11.5 \\
0.0 \\
19.2 \\
0.0 \\
53.8\end{array}$ & $\begin{array}{r}0.0 \\
0.0 \\
0.0 \\
18.8 \\
31.3 \\
0.0 \\
12.5 \\
0.0 \\
37.5\end{array}$ & $\begin{array}{r}0.0 \\
2.3 \\
0.0 \\
41.9 \\
32.0 \\
4.7 \\
0.0 \\
0.0 \\
18.6\end{array}$ & $\begin{array}{r}0.0 \\
0.0 \\
0.0 \\
62.5 \\
10.9 \\
15.6 \\
6.3 \\
0.0 \\
4.7\end{array}$ & $\begin{array}{r}0.0 \\
0.0 \\
0.0 \\
59.1 \\
9.1 \\
16.7 \\
3.0 \\
1.5 \\
10.6\end{array}$ & $\begin{array}{r}0.0 \\
0.0 \\
0.0 \\
79.2 \\
6.3 \\
6.3 \\
2.1 \\
0.0 \\
6.3\end{array}$ & $\begin{array}{r}0.0 \\
1.7 \\
0.0 \\
72.4 \\
1.7 \\
10.3 \\
3.4 \\
0.0 \\
10.3\end{array}$ & $\begin{array}{r}0.0 \\
0.0 \\
0.0 \\
44.8 \\
0.0 \\
19.0 \\
6.9 \\
0.00 \\
29.3\end{array}$ & $\begin{array}{l}0.0 \\
0.0 \\
0.0 \\
0.0 \\
0.0 \\
0.00 \\
0.00 \\
0.0 \\
0.0\end{array}$ & 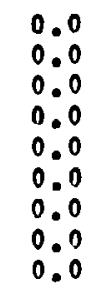 & $\begin{array}{lll}0 & 0 & 0 \\
0 & 0 & 0 \\
0.0 & 0 \\
0.0 & 0 \\
0 & 0 & 0 \\
0 & 0 & 0 \\
0 & 0 & 0 \\
0 & 0 & 0 \\
0 & 0 & 0\end{array}$ \\
\hline $\begin{array}{r}\text { rotel } \\
\text { runs: }\end{array}$ & 0 & 0 & 29 & 33 & 23 & 26 & 16 & 4.3 & 64 & 66 & 48 & 58 & 58 & 0 & 0 & 0 \\
\hline
\end{tabular}


Percentege of Runa of WPT 3 that Chenge to WPT ghown

27 Dearees North Lattude

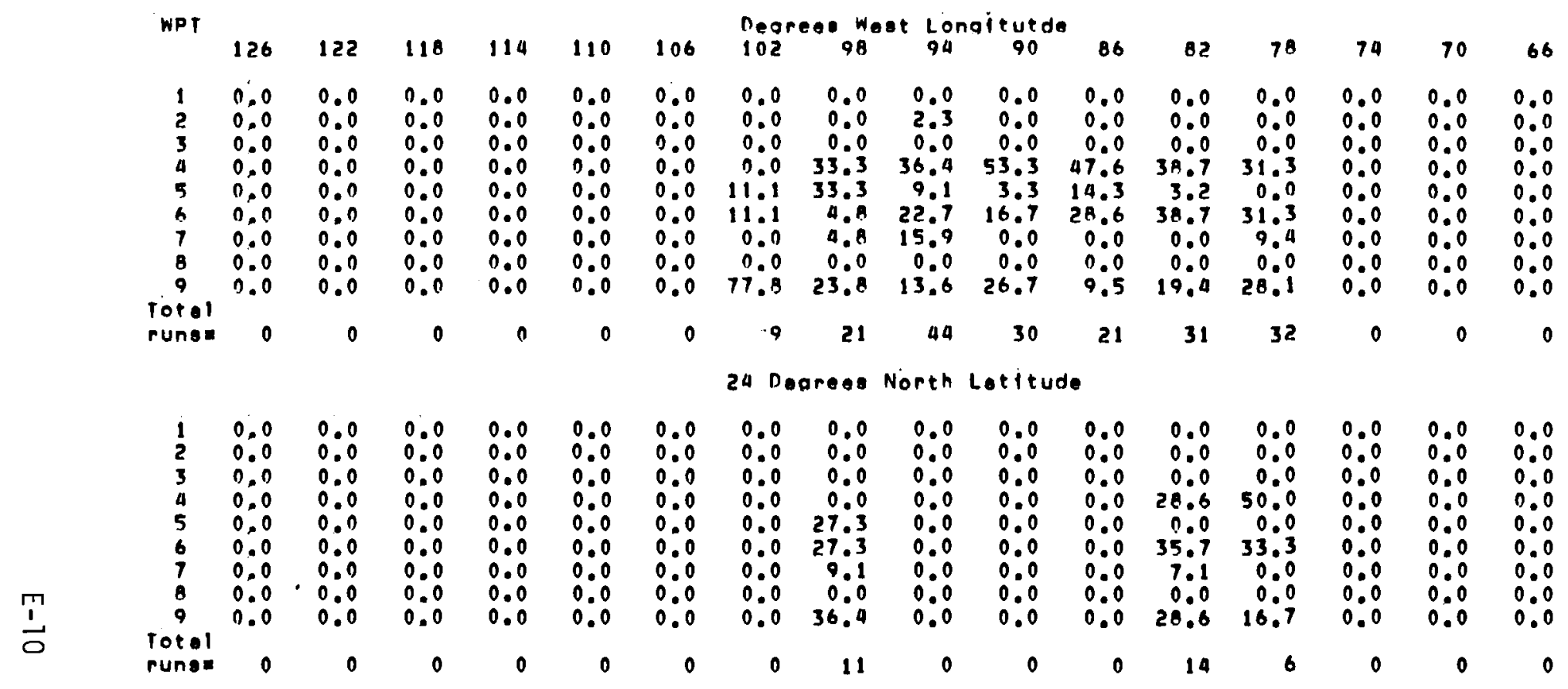


Percentade of Runs of WPT a that change to WPT shown

51 Dearees North Lotticude

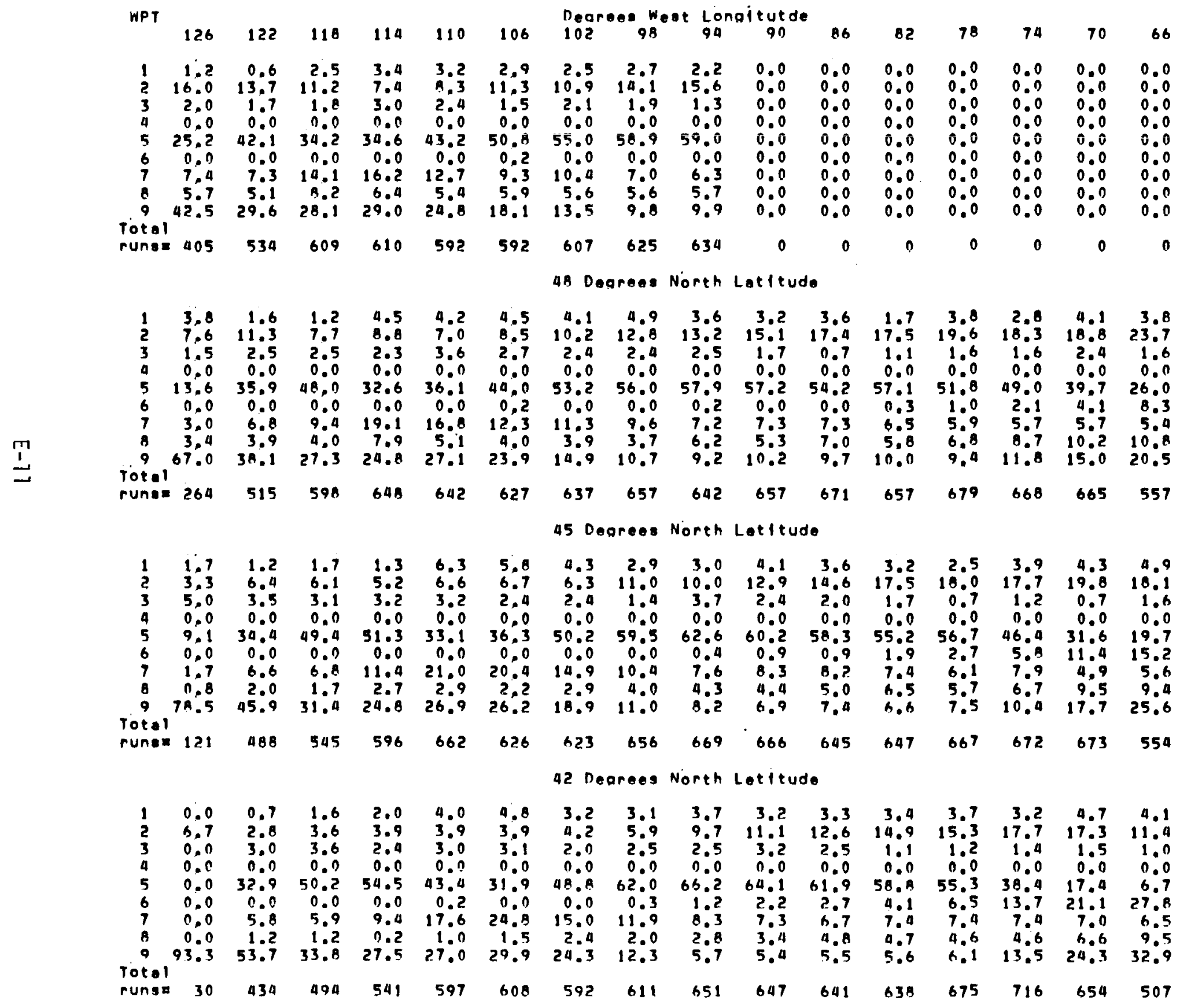


39 negrees North Lattude

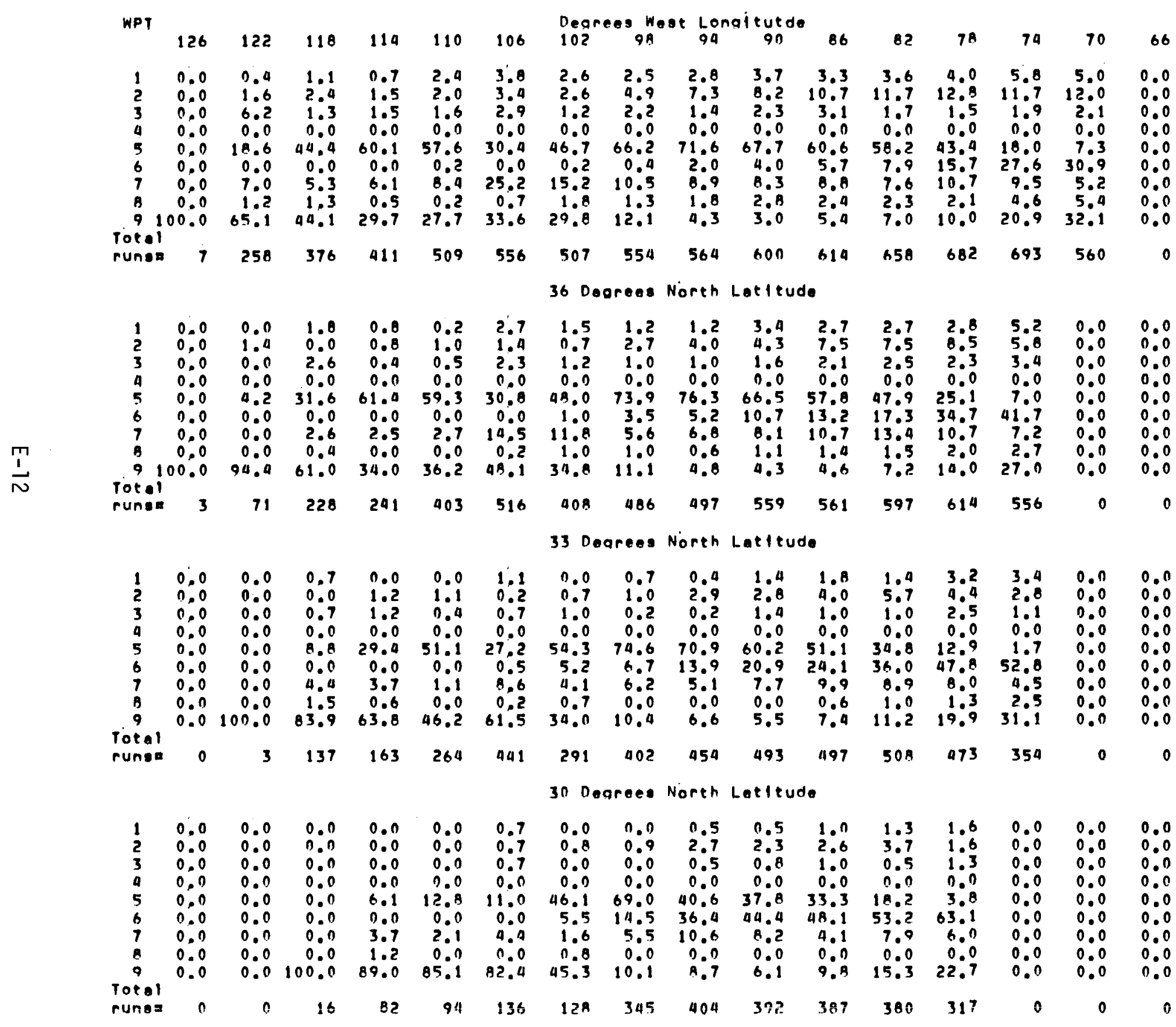


Porcentage of Rune of WPT a that Chonge to WPT shown

27 Dearees North Latitude

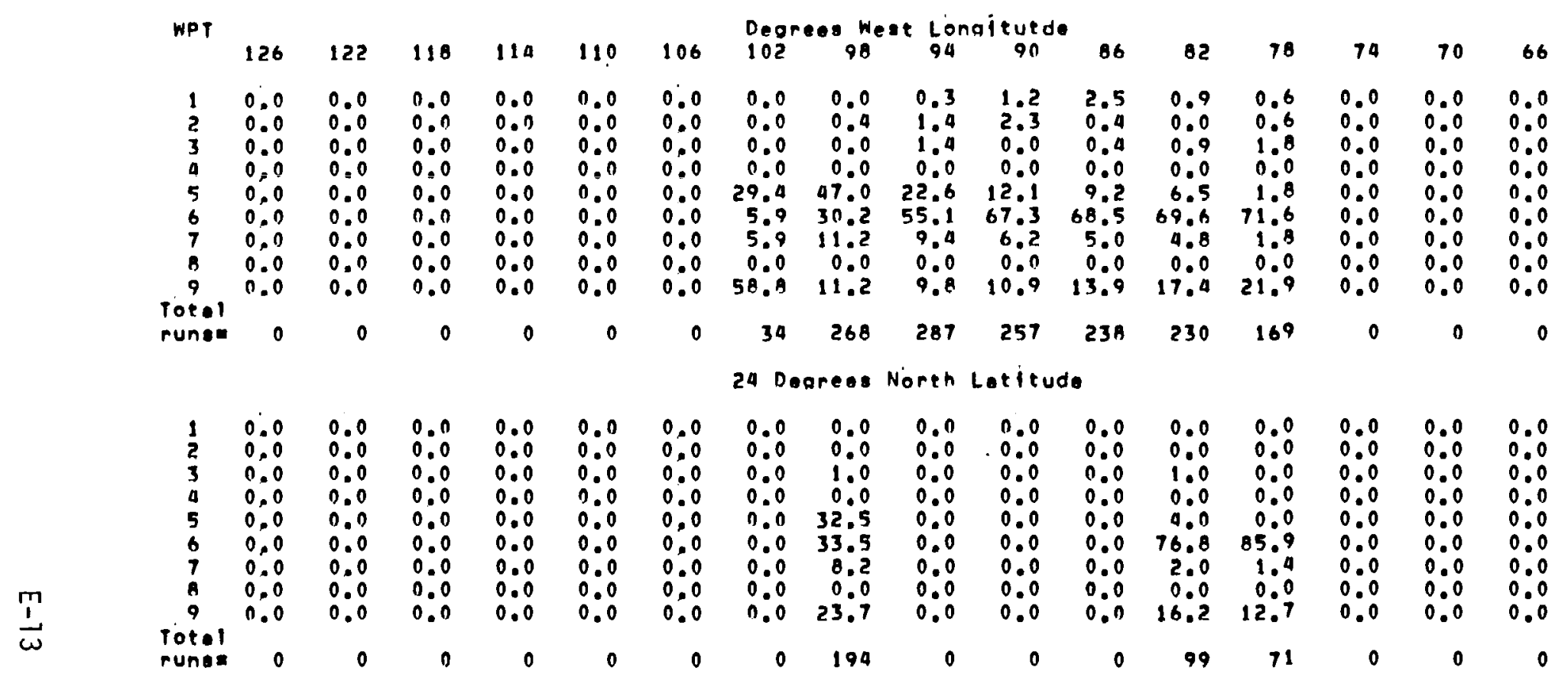




\section{Dearees North Lattude}

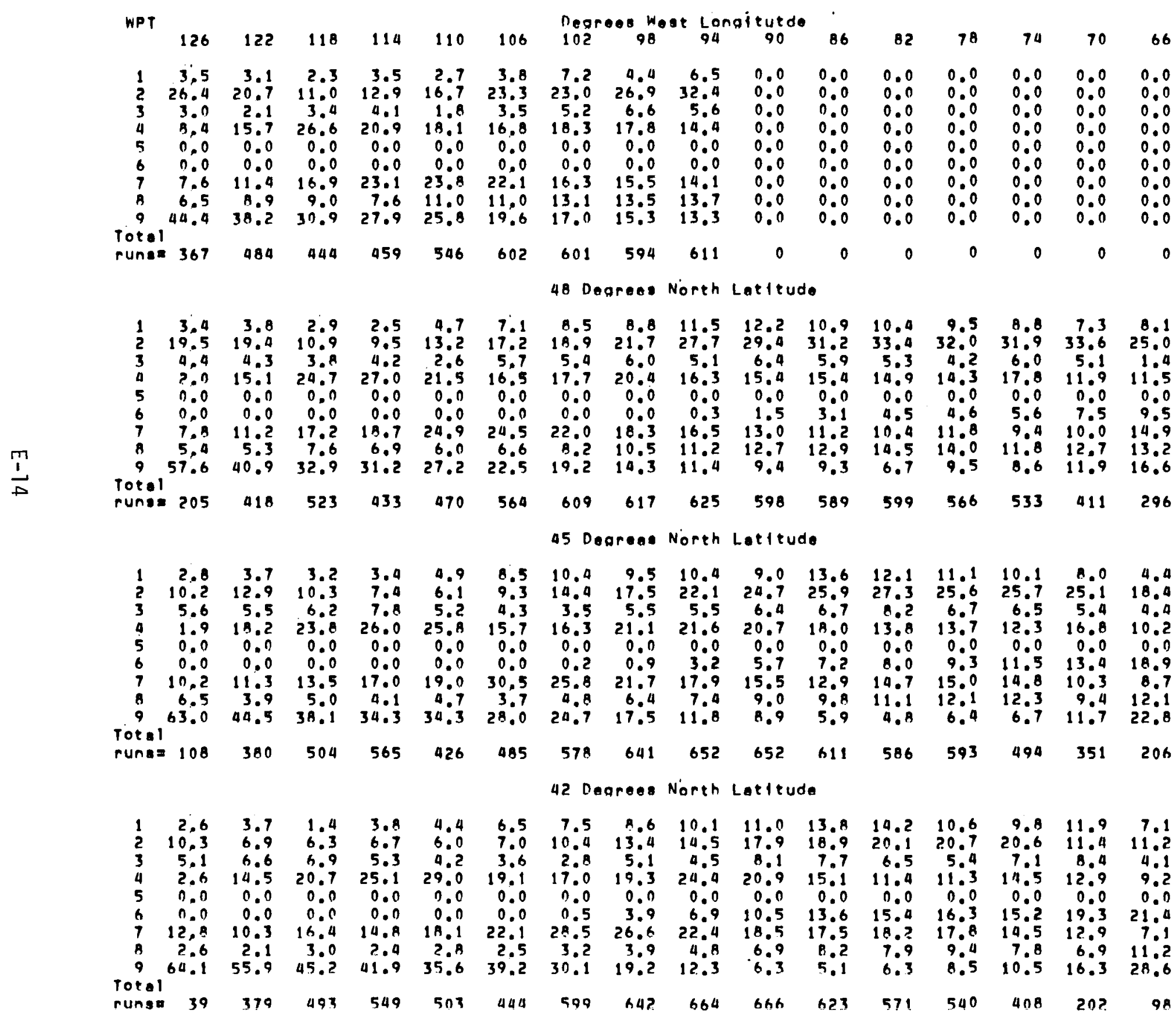


Percentage of Runs of WPT 5 thot Change to WPT shoun

39 Deareas North Latteude

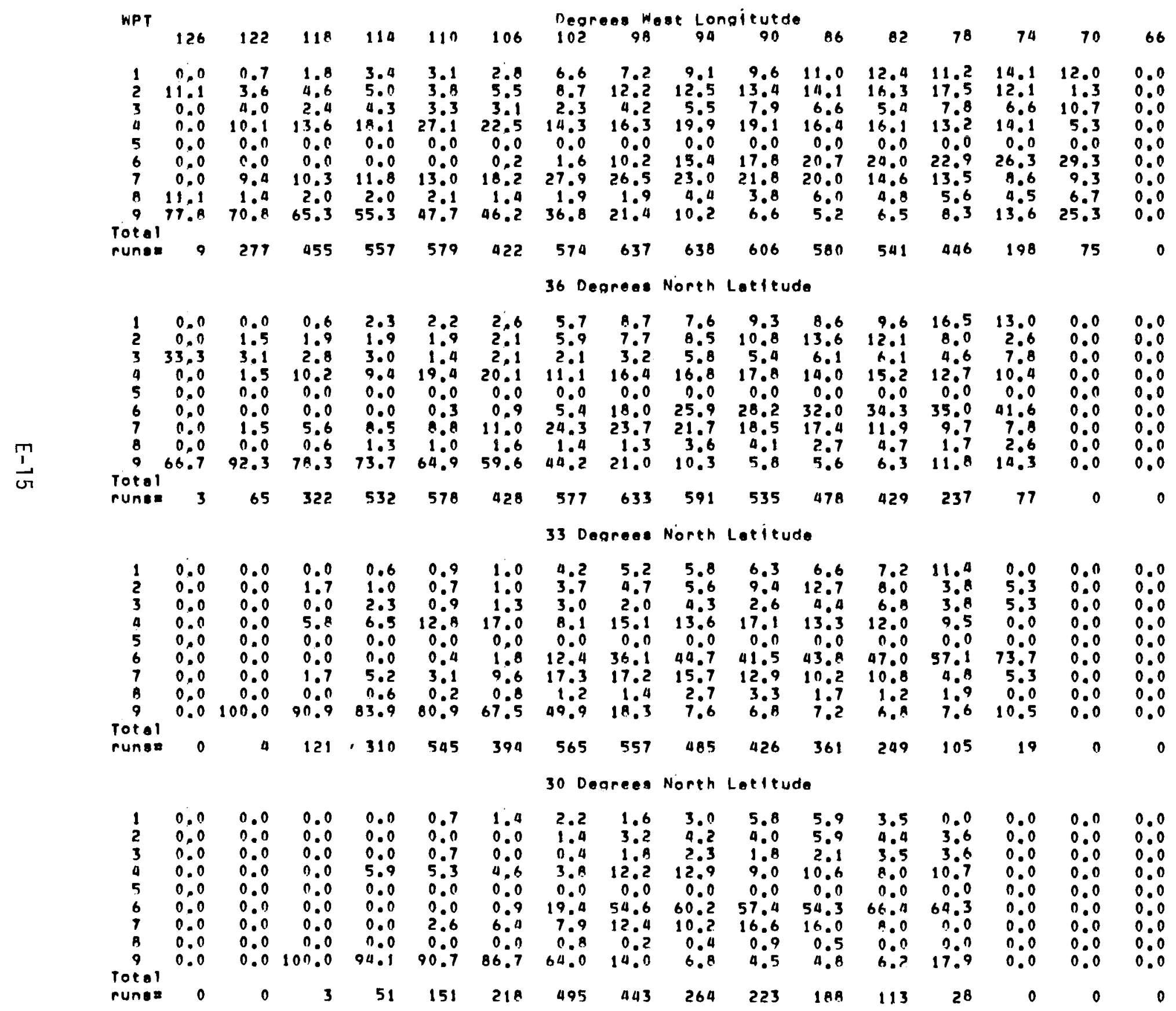


Percentage of Runs of WPT 5 that Change to WPT shown

27 Dearees North totitude

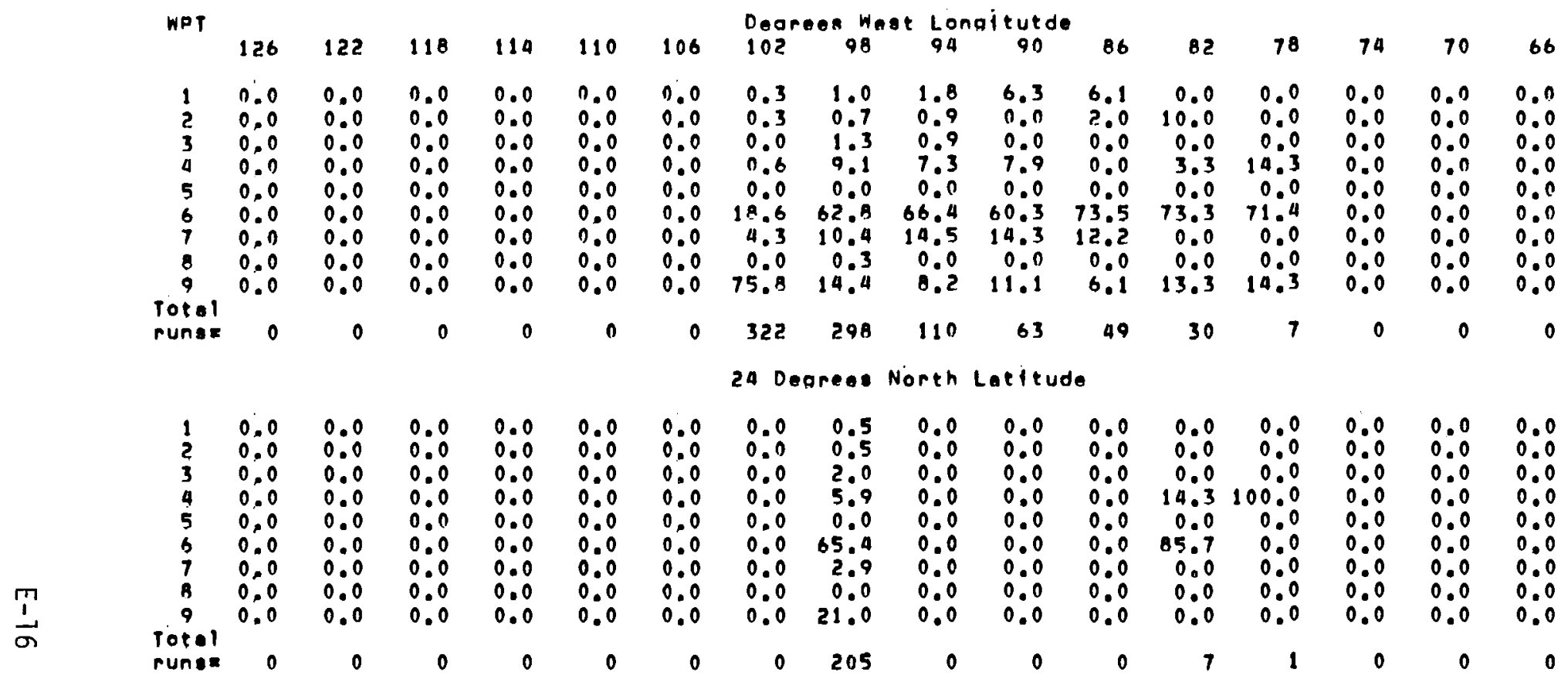


Percentage of Runs of WPT o that Change to WPT Shown

51 Dearees North Latteude

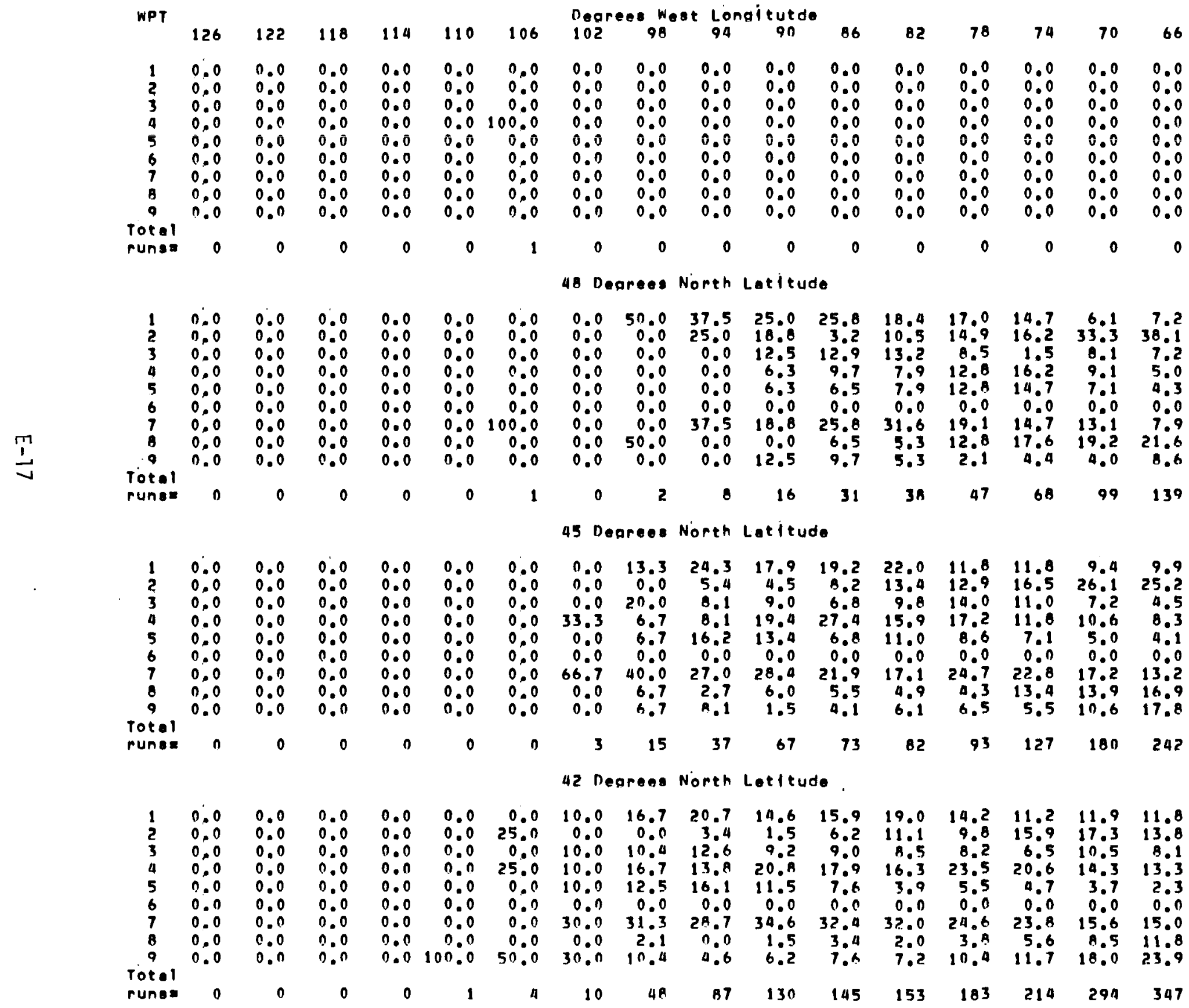


Pereentege of Runs of WPT 6 thet change to WPT shown

39 Dearees North Lotleude

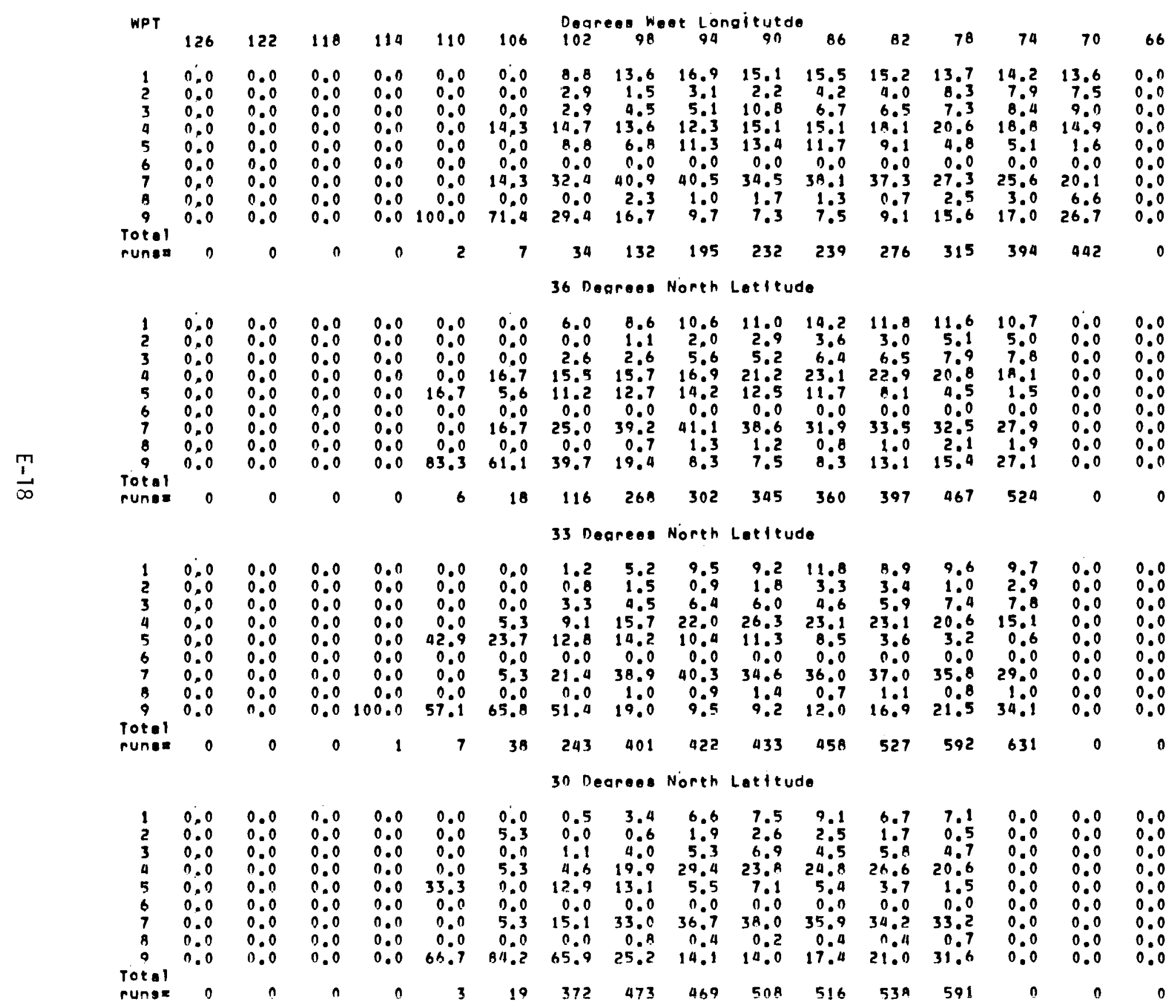


Percentege of Rune of WPT 6 thet chenge to WPT ghown

27 Degrees North Lattude

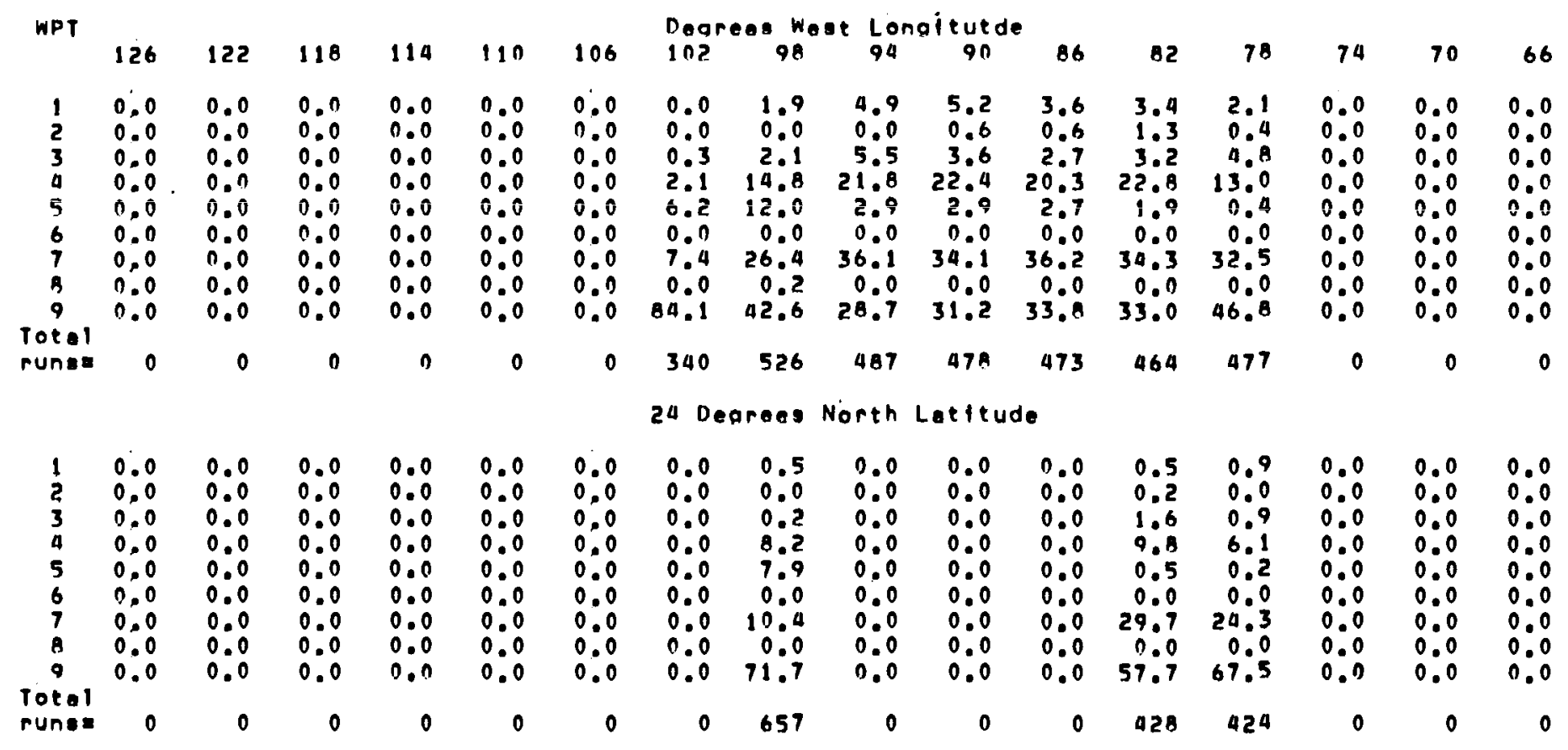


51 Degrees North Lettude

\begin{tabular}{|c|c|c|c|c|c|c|c|c|c|c|c|c|c|c|c|c|}
\hline WPT & 126 & 122 & 118 & 114 & 110 & 106 & $\begin{array}{l}\text { Degr } \\
102\end{array}$ & $=8$ & - $\begin{array}{l}\text { Lor } \\
94\end{array}$ & $\begin{array}{l}\text { tut de } \\
90\end{array}$ & 86 & 82 & 78 & 74 & 70 & 66 \\
\hline $\begin{array}{l}1 \\
2 \\
3 \\
4 \\
5 \\
6 \\
7 \\
8 \\
9\end{array}$ & $\begin{array}{r}4.7 \\
26.8 \\
1.6 \\
13.2 \\
13.7 \\
0.0 \\
0.0 \\
8.9 \\
31.1\end{array}$ & $\begin{array}{r}3.8 \\
20.5 \\
2.1 \\
20.9 \\
23.1 \\
0.0 \\
0.0 \\
6.4 \\
23.1\end{array}$ & $\begin{array}{r}4.2 \\
14.2 \\
3.3 \\
29.9 \\
22.1 \\
0.0 \\
0.0 \\
8.5 \\
17.8\end{array}$ & $\begin{array}{r}5.8 \\
14.2 \\
3.3 \\
28.2 \\
21.6 \\
0.0 \\
0.0 \\
9.4 \\
17.5\end{array}$ & $\begin{array}{r}6.0 \\
14.9 \\
6.0 \\
24.6 \\
23.8 \\
0.0 \\
0.0 \\
7.1 \\
17.5\end{array}$ & $\begin{array}{r}5.2 \\
15.0 \\
3.4 \\
25.1 \\
28.4 \\
0.0 \\
0.0 \\
6.1 \\
16.8\end{array}$ & $\begin{array}{r}5.6 \\
15.4 \\
3.9 \\
22.1 \\
31.9 \\
0.0 \\
0.0 \\
9.1 \\
13.0\end{array}$ & $\begin{array}{r}7.1 \\
19.7 \\
4.2 \\
28.2 \\
23.5 \\
0.0 \\
0.0 \\
6.7 \\
10.5\end{array}$ & $\begin{array}{r}6.0 \\
17.6 \\
5.0 \\
28.6 \\
24.6 \\
0.0 \\
0.0 \\
6.5 \\
11.6\end{array}$ & $\begin{array}{l}0.0 \\
0.0 \\
0.0 \\
0.0 \\
0.0 \\
0.0 \\
0.0 \\
0.0 \\
0.0\end{array}$ & $\begin{array}{l}0.0 \\
0.0 \\
0.0 \\
0.0 \\
0.0 \\
0.0 \\
0.0 \\
0.0 \\
0.0 \\
0.0\end{array}$ & $\begin{array}{l}0.0 \\
0.0 \\
0.0 \\
0.0 \\
0.0 \\
0.0 \\
0.0 \\
0.0 \\
0.0\end{array}$ & 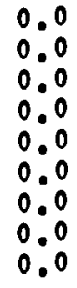 & $\begin{array}{l}0.0 \\
0.0 \\
0.0 \\
0.0 \\
0.0 \\
0.0 \\
0.0 \\
0.0 \\
0.0\end{array}$ & $\begin{array}{l}0.0 \\
0.0 \\
0.0 \\
0.0 \\
0.0 \\
0.0 \\
0.0 \\
0.0 \\
0.0 \\
0.0\end{array}$ & $\begin{array}{l}0.0 \\
0.0 \\
0.0 \\
0.0 \\
0.0 \\
0.0 \\
0.0 \\
0.0 \\
0.0\end{array}$ \\
\hline $\begin{array}{l}\text { otal } \\
\text { unas }\end{array}$ & 190 & 234 & 331 & 390 & 382 & 327 & 285 & 238 & 199 & 0 & 0 & 0 & 0 & 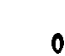 & 0 & \\
\hline
\end{tabular}

48 Dearees North Latitude

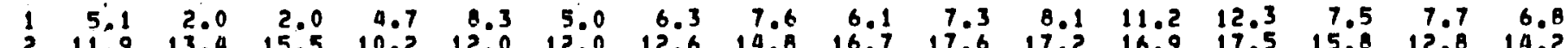

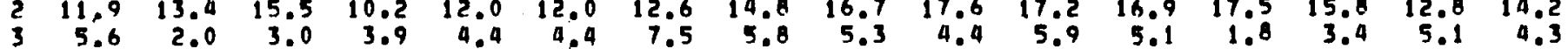

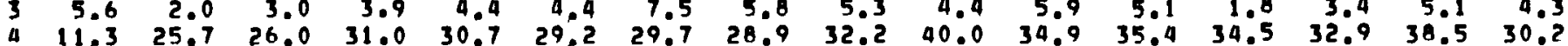

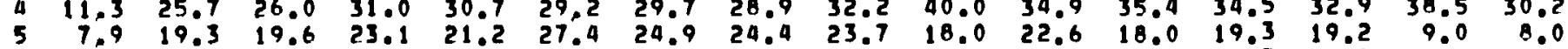

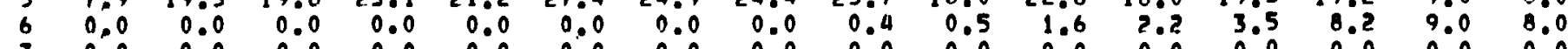

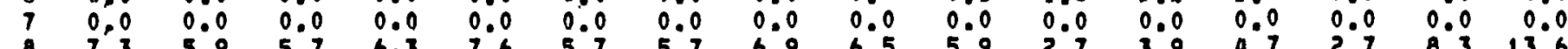

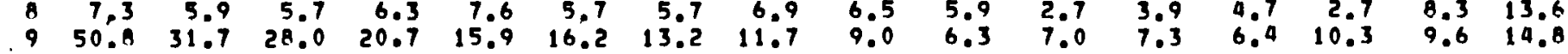

186

\section{Degrees North Letitude}

\begin{tabular}{|c|c|c|c|c|c|c|c|c|c|c|c|c|c|c|c|c|}
\hline $\begin{array}{c}1 \\
2 \\
3 \\
4 \\
5 \\
6 \\
7 \\
9 \\
9 \\
\text { Tot } 1 \\
\text { runs }\end{array}$ & $\begin{array}{r}3.1 \\
7.4 \\
4.9 \\
9.9 \\
4.3 \\
0.0 \\
0.0 \\
5.5 \\
69.9\end{array}$ & $\begin{array}{r}3.9 \\
10.1 \\
6.1 \\
25.7 \\
14.5 \\
0.0 \\
0.0 \\
5.6 \\
34.1\end{array}$ & $\begin{array}{r}5.2 \\
6.6 \\
4.2 \\
23.9 \\
18.8 \\
0.0 \\
0.0 \\
7.0 \\
34.3\end{array}$ & $\begin{array}{r}2.6 \\
7.2 \\
3.3 \\
30.1 \\
24.2 \\
0.0 \\
0.0 \\
2.3 \\
30.4\end{array}$ & $\begin{array}{r}6.3 \\
6.5 \\
2.9 \\
36.6 \\
21.1 \\
0.0 \\
0.0 \\
4.02 \\
22.5\end{array}$ & $\begin{array}{r}8.2 \\
9.8 \\
5.3 \\
29.4 \\
26.0 \\
0.0 \\
0.0 \\
3.3 \\
18.0\end{array}$ & $\begin{array}{r}9.0 \\
11.7 \\
5.4 \\
27.2 \\
27.9 \\
0.2 \\
0.0 \\
3.3 \\
16.4\end{array}$ & $\begin{array}{r}6.6 \\
11.3 \\
4.9 \\
32.1 \\
25.4 \\
1.2 \\
0.0 \\
5.2 \\
13.3\end{array}$ & $\begin{array}{r}7.2 \\
14.5 \\
5.8 \\
36.6 \\
23.6 \\
0.7 \\
0.0 \\
5.1 \\
6.5\end{array}$ & $\begin{array}{r}8.9 \\
12.3 \\
7.6 \\
30.5 \\
20.0 \\
4.7 \\
0.0 \\
5.1 \\
6.0\end{array}$ & $\begin{array}{r}10.5 \\
15.5 \\
4.5 \\
36.8 \\
21.4 \\
3.6 \\
0.0 \\
3.2 \\
4.5 \\
220\end{array}$ & $\begin{array}{r}10.7 \\
12.1 \\
5.4 \\
30.8 \\
22.8 \\
2.2 \\
0.0 \\
6.3 \\
5.8\end{array}$ & $\begin{array}{r}10.0 \\
14.9 \\
5.9 \\
31.7 \\
20.8 \\
4.1 \\
0.0 \\
6.3 \\
6.3\end{array}$ & $\begin{array}{r}7.5 \\
14.2 \\
5.3 \\
37.2 \\
18.6 \\
7.1 \\
0.0 \\
3.5 \\
6.6\end{array}$ & $\begin{array}{r}8.7 \\
12.7 \\
2.9 \\
35.3 \\
11.6 \\
8.1 \\
0.0 \\
6.4 \\
14.5\end{array}$ & $\begin{array}{r}8.2 \\
12.9 \\
5.3 \\
28.2 \\
3.5 \\
13.5 \\
0.0 \\
8.8 \\
19.4\end{array}$ \\
\hline & & & & & & & 42 & & & 10 ts & & & & & & \\
\hline $\begin{array}{l}1 \\
2 \\
3 \\
4 \\
5 \\
6 \\
7 \\
8 \\
9 \\
0+1\end{array}$ & $\begin{array}{r}4.9 \\
4.9 \\
4.9 \\
1.6 \\
0.5 \\
0.0 \\
0.0 \\
5.5 \\
77.5\end{array}$ & $\begin{array}{r}3.8 \\
8.8 \\
3.8 \\
24.2 \\
15.9 \\
0.0 \\
0.0 \\
0.9 \\
38.5\end{array}$ & $\begin{array}{r}1.9 \\
3.8 \\
2.8 \\
26.5 \\
23.2 \\
0.0 \\
0.0 \\
4.3 \\
37.4\end{array}$ & $\begin{array}{r}2.5 \\
6.5 \\
2.9 \\
27.7 \\
20.9 \\
0.0 \\
0.0 \\
2.2 \\
37.4\end{array}$ & $\begin{array}{r}3.6 \\
7.7 \\
1.9 \\
31.1 \\
24.6 \\
0.0 \\
0.0 \\
1.9 \\
29.2\end{array}$ & $\begin{array}{r}5.2 \\
9.2 \\
2.8 \\
30.7 \\
24.4 \\
0.2 \\
0.0 \\
3.1 \\
24.4\end{array}$ & $\begin{array}{r}6.7 \\
9.3 \\
4.0 \\
29.9 \\
29.7 \\
0.7 \\
0.0 \\
1.8 \\
19.0\end{array}$ & $\begin{array}{r}7.7 \\
7.7 \\
5.5 \\
36.1 \\
24.4 \\
1.7 \\
0.0 \\
2.7 \\
14.2\end{array}$ & $\begin{array}{r}9.8 \\
9.1 \\
6.1 \\
3 n .1 \\
22.6 \\
4.0 \\
0.0 \\
4.9 \\
10.4\end{array}$ & $\begin{array}{r}9.5 \\
9.8 \\
5.3 \\
36.5 \\
22.1 \\
8.8 \\
0.0 \\
3.9 \\
4.2\end{array}$ & $\begin{array}{r}8 . A \\
11.1 \\
9.6 \\
41.2 \\
18.7 \\
7.6 \\
0.0 \\
3.1 \\
5.0\end{array}$ & $\begin{array}{r}11.4 \\
14.0 \\
3.3 \\
37.1 \\
19.1 \\
6.6 \\
0.0 \\
4.4 \\
4.0\end{array}$ & $\begin{array}{r}9.7 \\
11.7 \\
4.8 \\
36.7 \\
17.7 \\
11.3 \\
0.0 \\
3.6 \\
4.4\end{array}$ & $\begin{array}{r}8.3 \\
9.6 \\
5.2 \\
44.3 \\
11.7 \\
12.2 \\
0.0 \\
2.6 \\
6.1\end{array}$ & $\begin{array}{r}7.9 \\
3.8 \\
3.9 \\
38.2 \\
7.0 \\
15.8 \\
0.0 \\
5.7 \\
12.7\end{array}$ & $\begin{array}{r}30.4 \\
2.1 \\
17.0 \\
0.0 \\
7.6 \\
23.7\end{array}$ \\
\hline $\ln 8 x$ & 182 & 182 & 211 & 278 & 366 & 459 & $\triangle 4 B$ & 402 ? & 328 & 285 & 262 & 772 & 248 & 230 & $22 \mathrm{~A}$ & 190 \\
\hline
\end{tabular}


Percentage of Runa of WPT 1 thet Change to WPT stiown

39 Degrees North Latitude

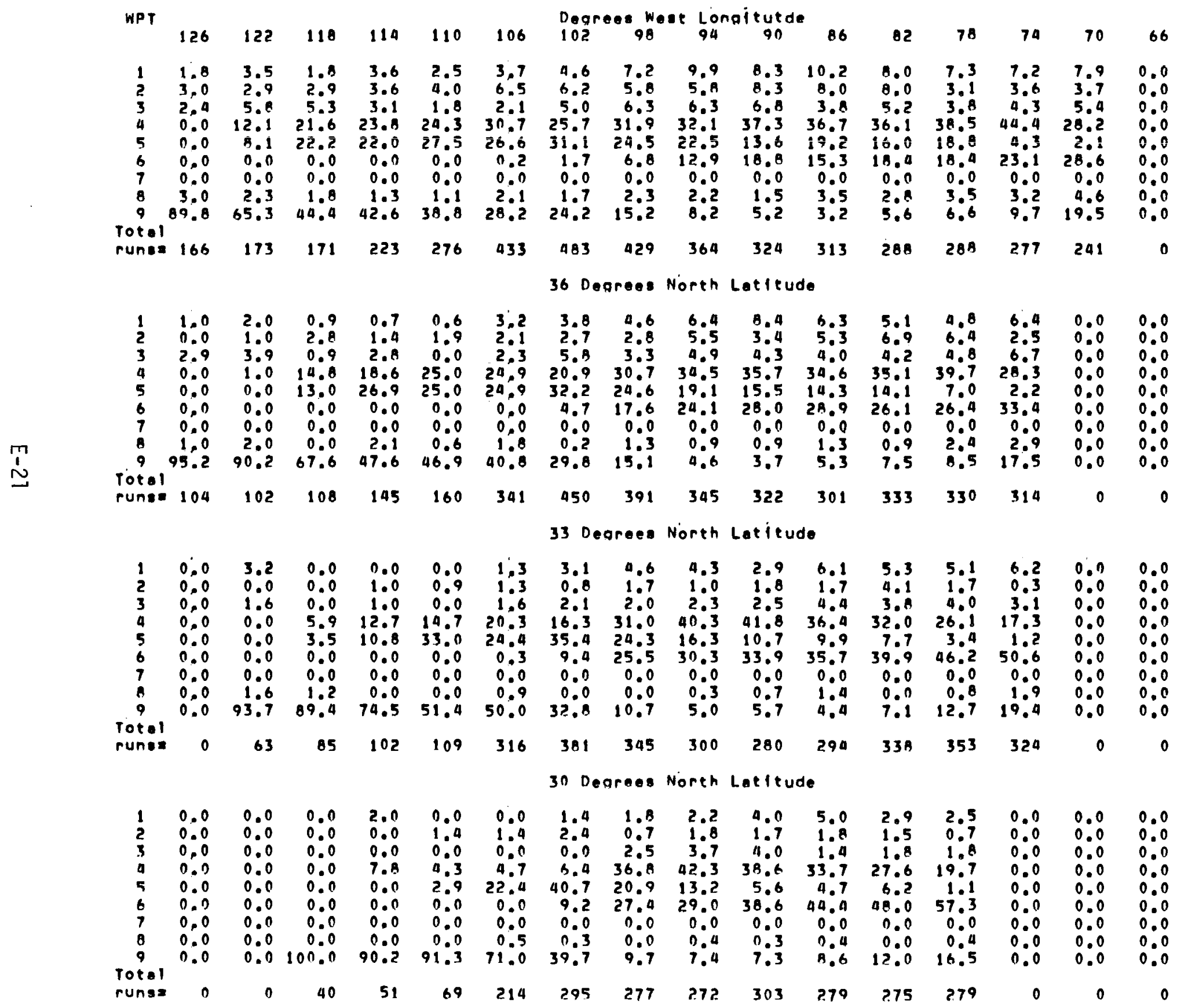


Porcentage of Runs of WPT 7 thet change to WPT shown

27 Dearees North Lattude

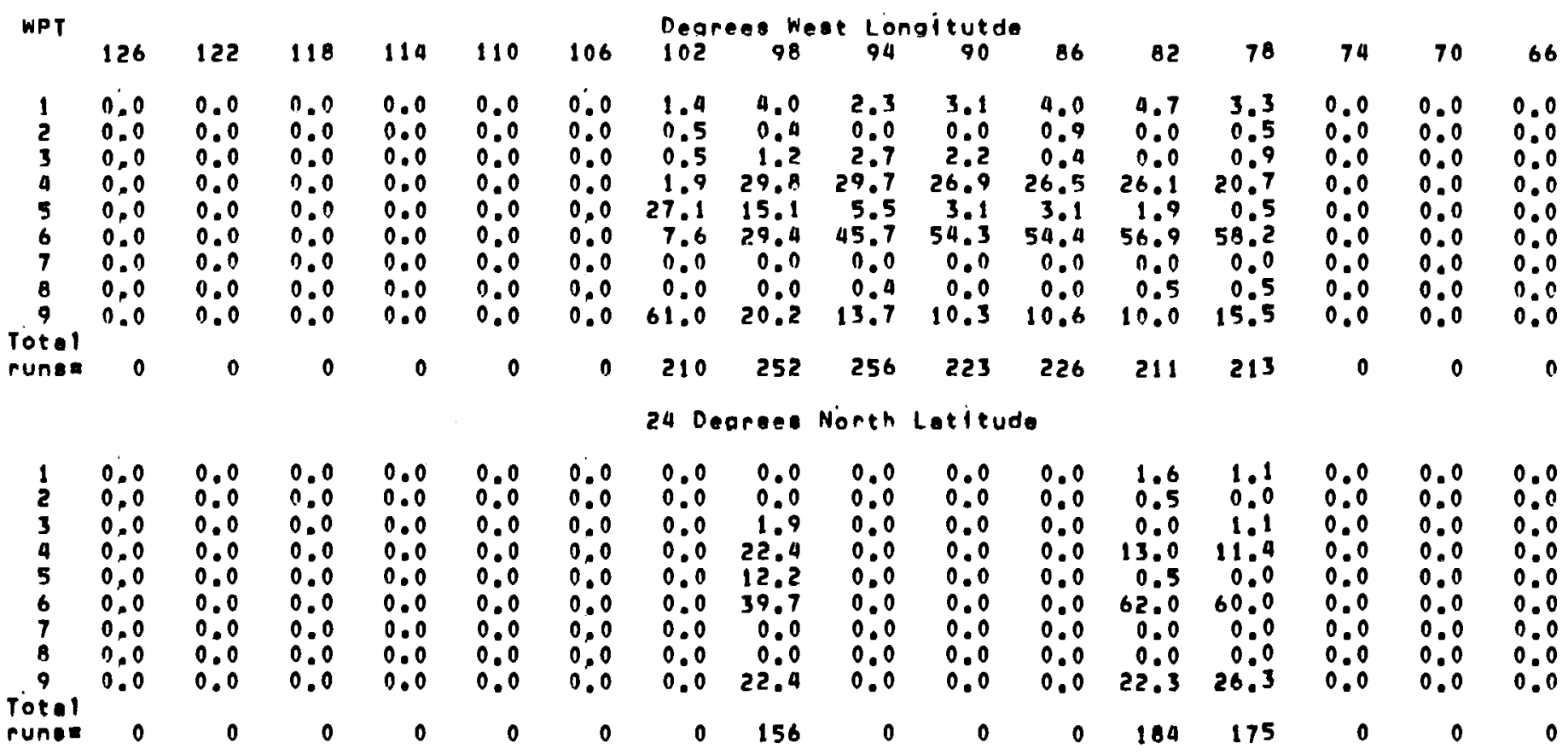


Percentage of Rune of WPT 6 thet Change to WPT shown

51 Degreas North Lat ltude

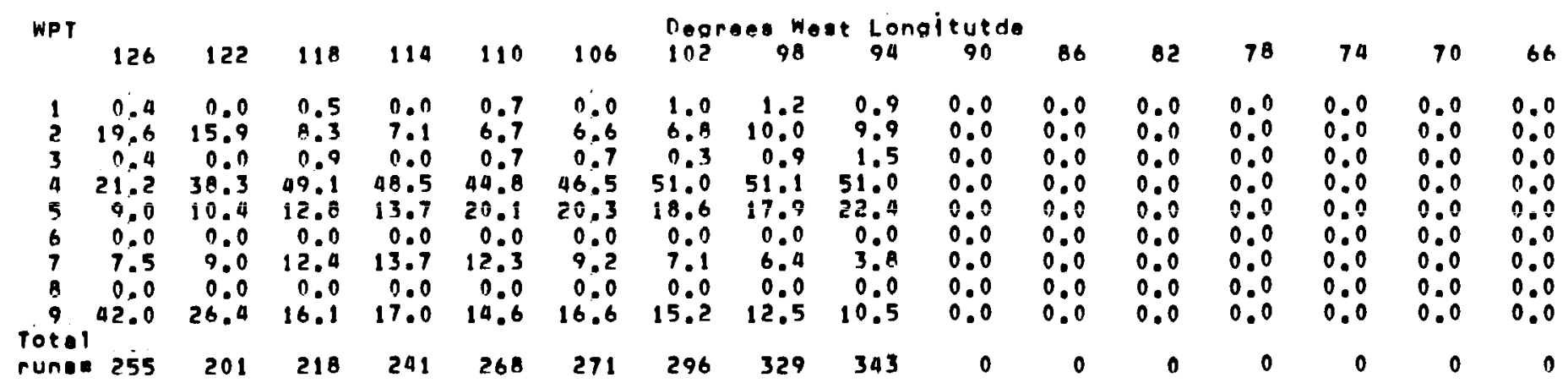

48 Degrees North Latltude

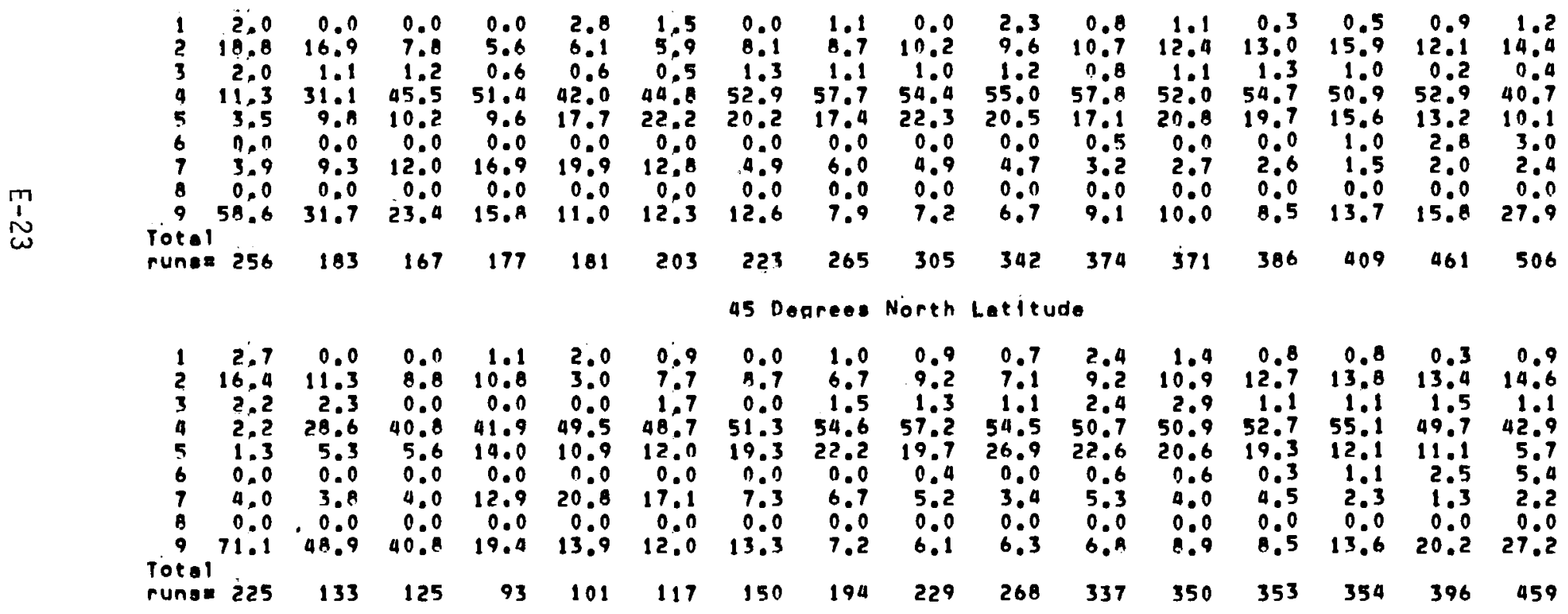
az Dearees North Lattude

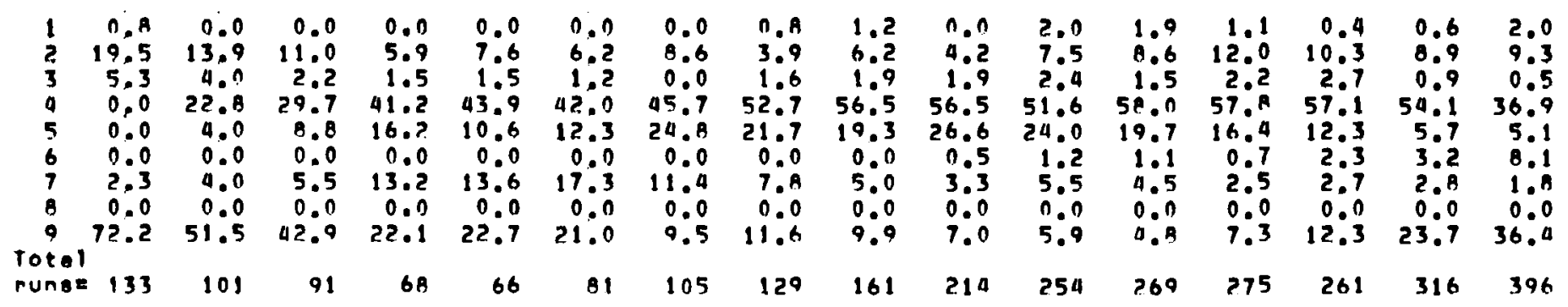


39 Degrees North Latitude

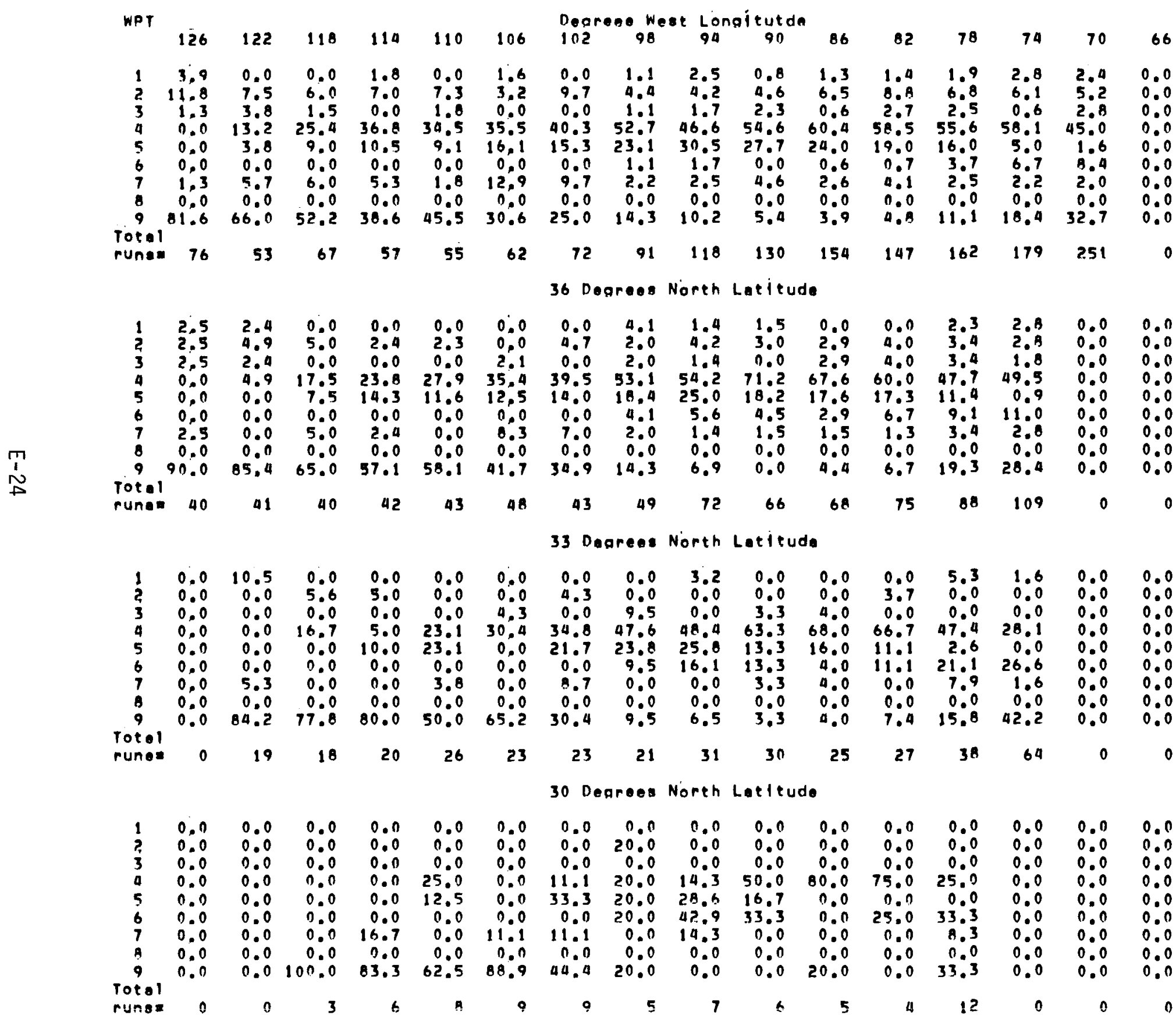


Parcentage of Runa of WPT g that Change to WPT shown 27 Degrees North Lattude

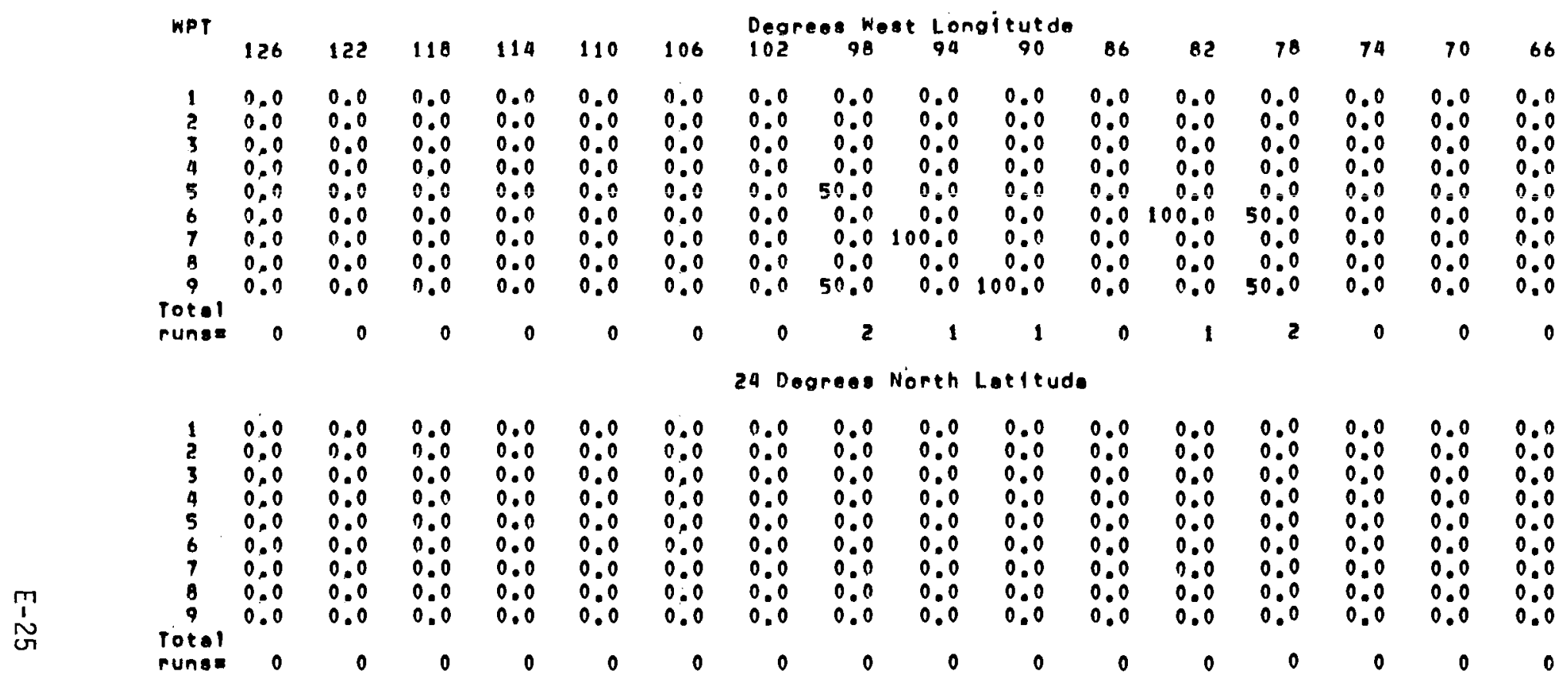


Percentage of Runs of WPT 9 that Change to WPT shown

51 Degrees North Lotitude

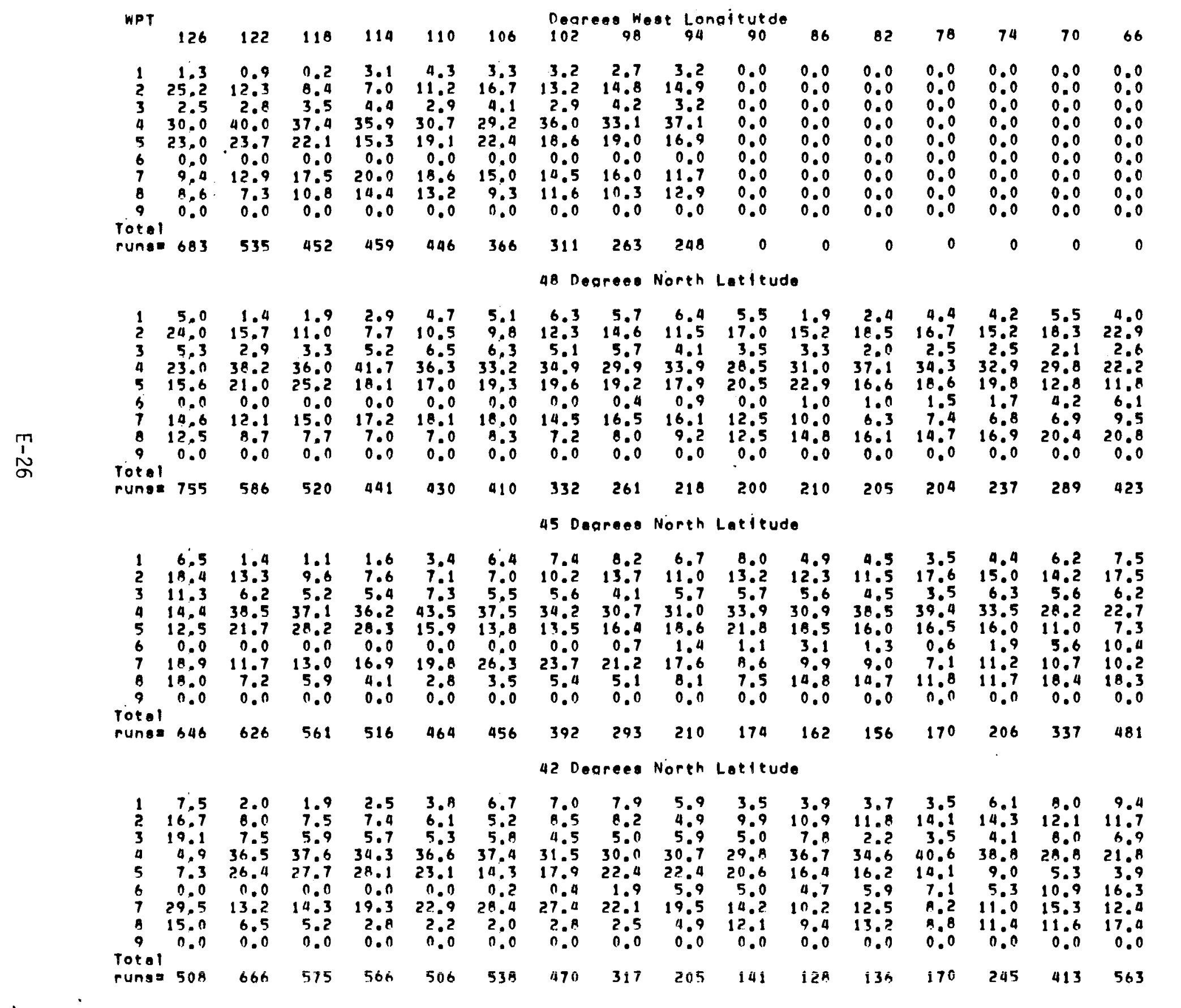


39 Degroes North Latteude

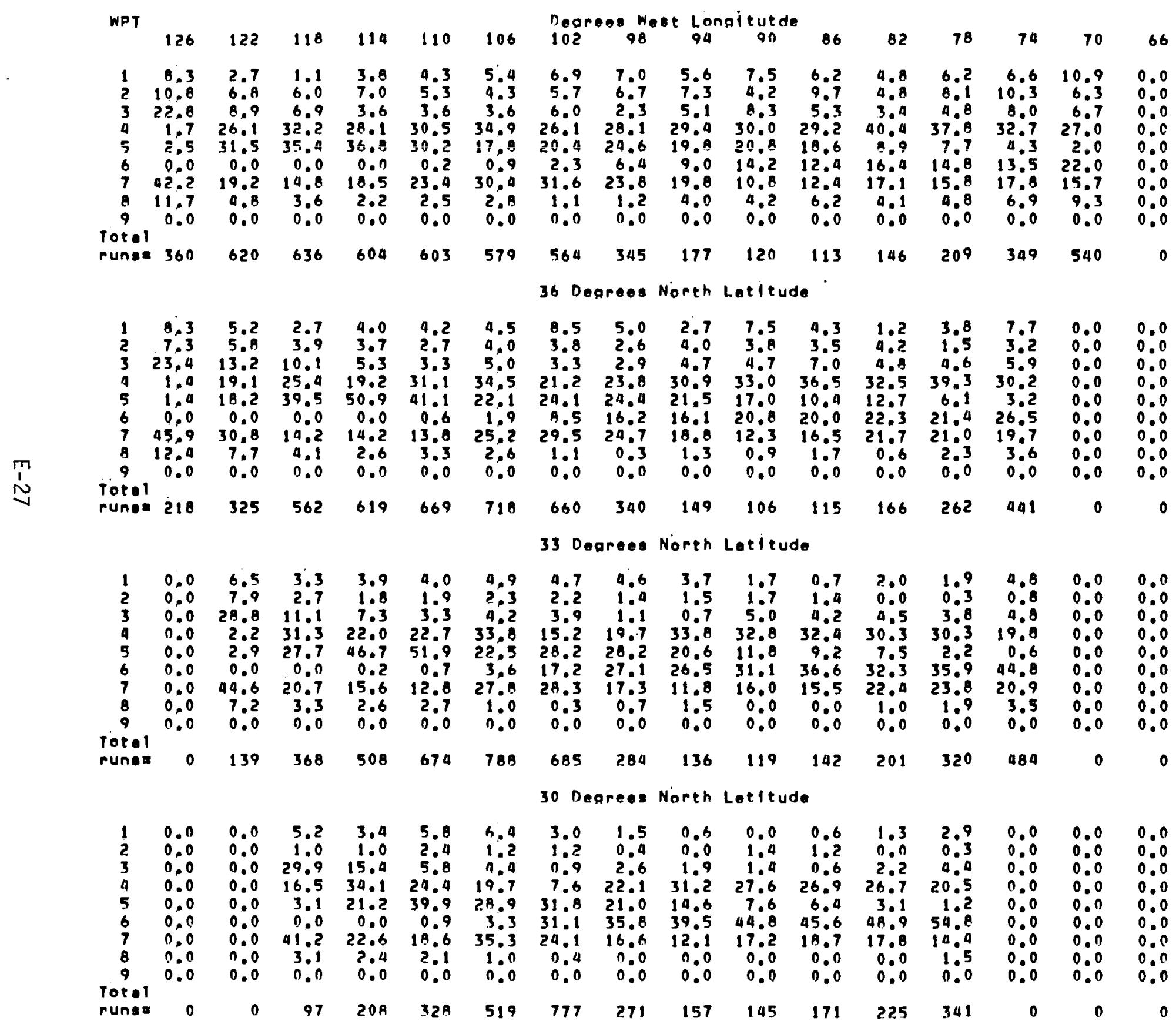


Percentege of Runs of WPT 9 that change to WPT shown

27 Degrees North Latt tude

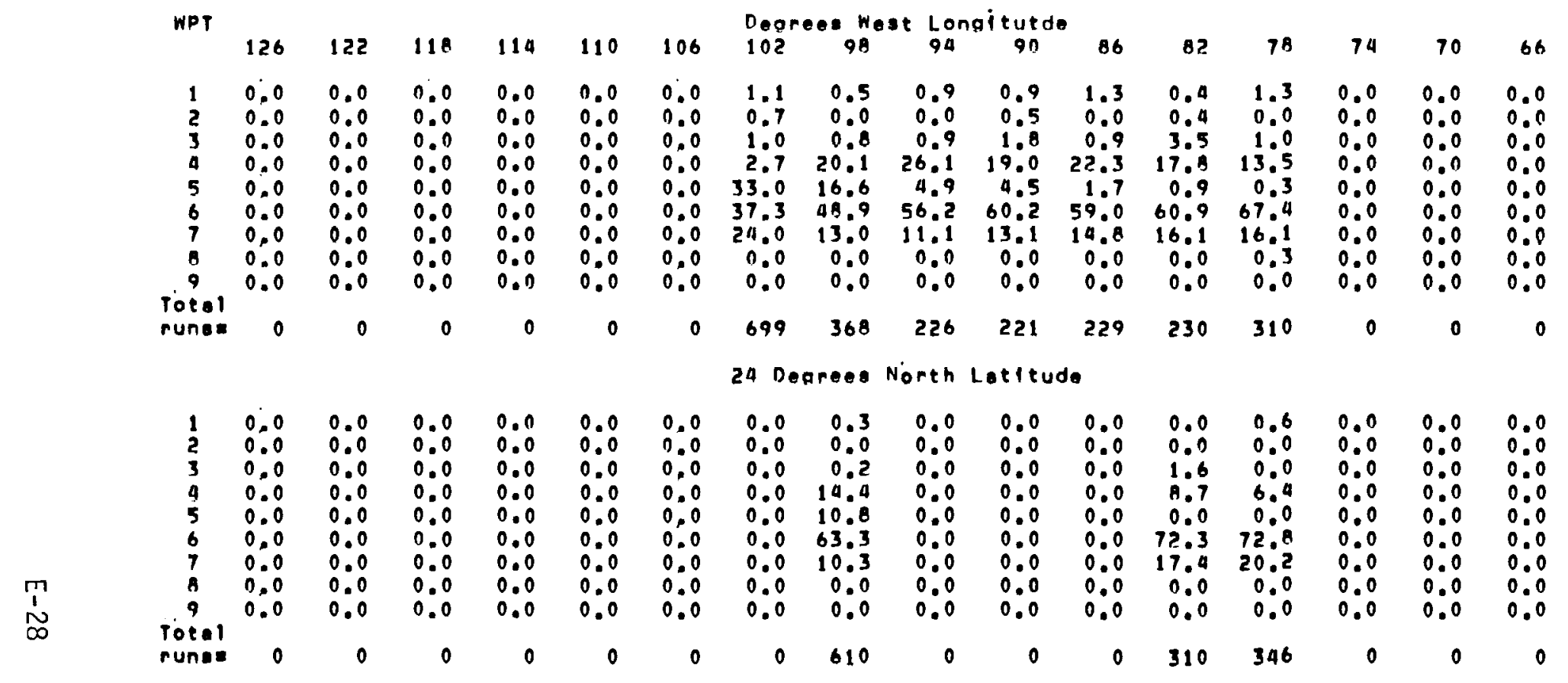


summery of alternation Probabilite

$$
\text { Northwat }
$$

\begin{tabular}{|c|c|c|c|c|c|c|c|c|c|}
\hline $\begin{array}{l}\text { New } \\
\text { WPT }\end{array}$ & 1 & $\bar{z}$ & $\begin{array}{c}\text { Woather } \\
3\end{array}$ & Pate & $=\frac{1}{5}$ & of ${ }_{6}^{\mathrm{Cu}}$ & rent R & Qun & 9 \\
\hline 1 & 0.0 & 1.7 & 1.2 & 2.8 & 3.7 & 0.0 & 4.5 & 0.8 & 3.4 \\
\hline 2 & 6.5 & $0: 0$ & 6.0 & 6.8 & 11.1 & 6.3 & 10.0 & 10.5 & 10.7 \\
\hline 3 & 8.1 & 3.7 & 0.0 & 2.6 & 4.3 & 0.0 & 3.6 & 1.2 & 6.2 \\
\hline 9 & 33.5 & 25.2 & 39.0 & 0.0 & 19.9 & 18.8 & 25.6 & 34.4 & 32.0 \\
\hline 5 & 8.9 & 9.8 & 13.0 & 40.0 & 0.0 & 0.0 & 21.0 & 11.0 & 22.0 \\
\hline 6 & 0.1 & 0.0 & 0.0 & 0.0 & 0.0 & 0.0 & 0.0 & 0.0 & 0.0 \\
\hline 7 & 15.5 & $11: 8$ & 7.7 & 12.5 & 16.9 & 12.5 & 0.0 & 10.2 & 16.5 \\
\hline 8 & 7.0 & 23.9 & 4.4 & 3.3 & 5.1 & 0.0 & 4.9 & 0.0 & 7.2 \\
\hline 9 & $20^{\circ} .4$ & $23^{\circ} .8$ & 28.7 & 32.0 & 39.0 & 62.5 & $3 n .3$ & 32.2 & 0.0 \\
\hline $\begin{array}{l}\text { Totel } \\
\text { rung }=\end{array}$ & 1978 & $57 \geq 0$ & 2653 & 14495 & 12689 & 16 & 8560 & 4325 & 16113 \\
\hline
\end{tabular}

Summary of Alternation Probability Noreh Ceneral

\begin{tabular}{|c|c|c|c|c|c|c|c|c|c|}
\hline $\begin{array}{l}\text { NeW } \\
\text { WPT }\end{array}$ & 1 & 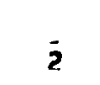 & $\begin{array}{c}\text { Weethe } \\
3\end{array}$ & r Pette & orn Troe & of ${ }_{6}^{\mathrm{Cu}}$ & $\operatorname{rant} R$ & Run & 9 \\
\hline 1 & 0.0 & 3.2 & 1.8 & 3.3 & 8.9 & 16.3 & 7.3 & 0.9 & 6.2 \\
\hline 2 & $5: 7$ & 0.0 & 6.4 & 10.0 & 19.0 & 2.9 & 11.0 & 7.9 & 10.4 \\
\hline 3 & 13.3 & 6.9 & 0.0 & 2.2 & 5.4 & 8.4 & 5.5 & 1.2 & 0.0 \\
\hline 4 & 37.4 & $32^{\circ} .5$ & 96.4 & 0.0 & 18.6 & 14.7 & 31.3 & 53.2 & 31.4 \\
\hline 5 & 10.6 & 10.9 & 19.5 & 59.2 & 0.0 & 11.6 & 24.8 & 21.6 & 19.3 \\
\hline 6 & $3: 3$ & 0.4 & 0.6 & 0.6 & 4.2 & 0.0 & 3.5 & 0.1 & 2.0 \\
\hline 7 & $13: 0$ & 7.4 & 8.9 & 9.7 & 20.6 & 34.9 & 0.0 & 5.4 & 19.9 \\
\hline$B$ & 8.5 & $31: 0$ & 8.5 & 3.7 & 7.4 & 1.9 & 4.1 & 0.0 & 6.1 \\
\hline 9 & 7.4 & 7.6 & 7.9 & 11.3 & 15.9 & 9.4 & 12.5 & 9.6 & 0.0 \\
\hline $\begin{array}{l}\text { otel } \\
\text { uns }=\end{array}$ & 2625 & 5282 & 2327 & 11799 & 11004 & 1016 & 6144 & 3964 & 5241 \\
\hline
\end{tabular}




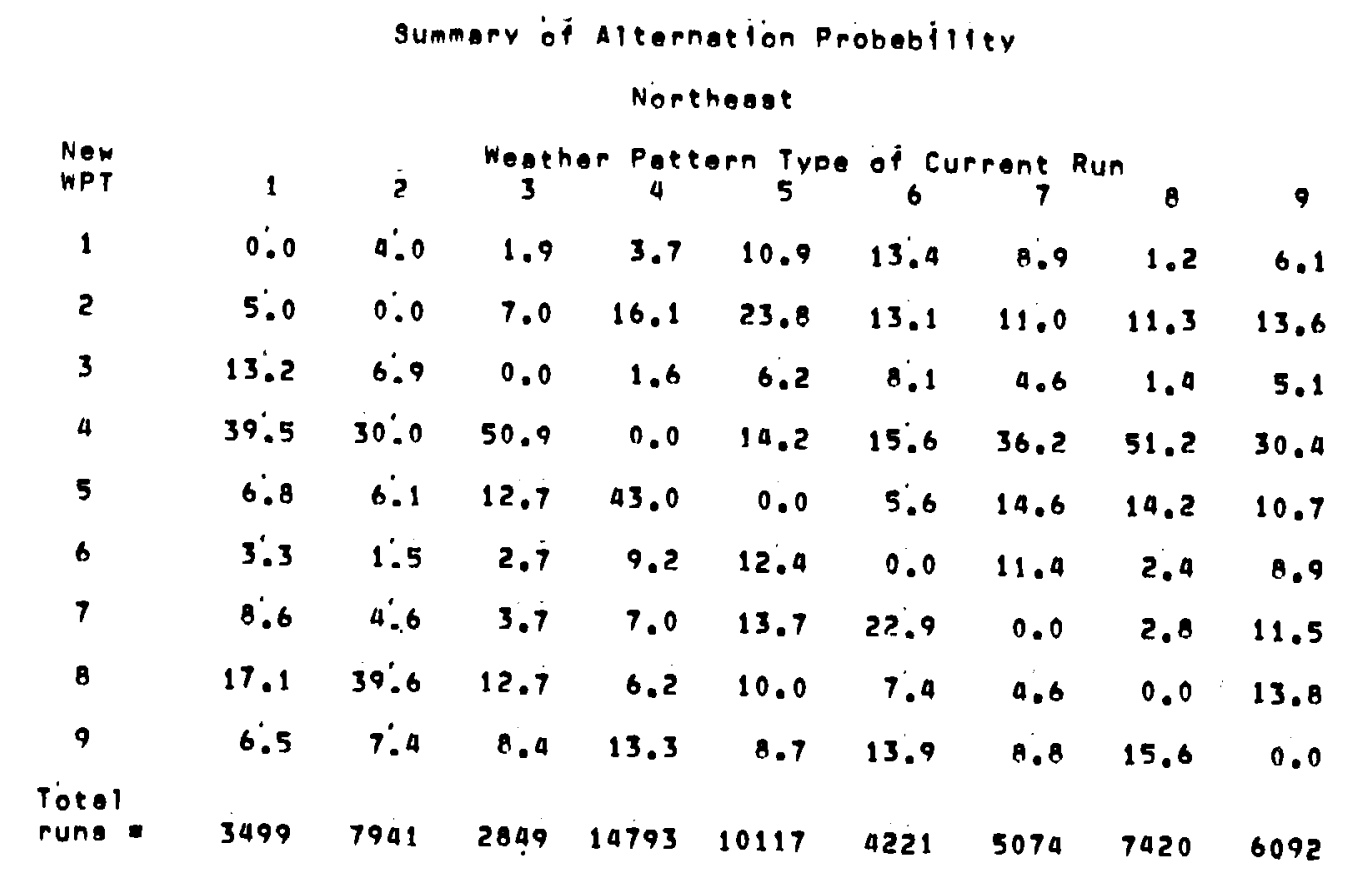

summerv of alternation Probebility

\begin{tabular}{|c|c|c|c|c|c|c|c|c|c|}
\hline \multirow[b]{2}{*}{$\begin{array}{l}\text { New } \\
\text { WPT }\end{array}$} & \multicolumn{9}{|c|}{ Southwast } \\
\hline & 1 & $\bar{z}$ & $\begin{array}{c}\text { Weother } \\
3\end{array}$ & $\begin{array}{l}\text { Pottern } \\
4\end{array}$ & $\begin{array}{l}\text { n Tyoe } \\
5\end{array}$ & of $C_{6}$ & $\begin{array}{r}\text { Eurrant } \\
7\end{array}$ & Run 8 & 9 \\
\hline 1 & 0.0 & 2.5 & 0.4 & 1.0 & 1.4 & 0.0 & 1.2 & 1.0 & 4.5 \\
\hline 2 & 0.2 & 0.0 & 1.1 & 0.8 & $1: 3$ & $1: 1$ & 1.3 & 2.3 & 3.0 \\
\hline 3 & 11.0 & 3.1 & $0 . n$ & 1.0 & 1.7 & 0.0 & 1.3 & 1.0 & 7.7 \\
\hline 4 & 21.1 & 13.1 & 19.9 & 0.0 & 12.5 & 6.5 & 14.1 & 17.4 & 25.2 \\
\hline 5 & 8.2 & 5.6 & 10.2 & $3 a .6$ & 0.0 & 16.3 & 17.7 & 7.5 & 33.1 \\
\hline 6 & $0 \div 0$ & 0.0 & 0.0 & 0.1 & 0.5 & 0.0 & 0.0 & 0.0 & 1.1 \\
\hline 7 & $8: 0$ & 6.2 & 4.0 & 5.8 & 6.8 & 6.5 & 0.0 & 3.1 & 22.2 \\
\hline 8 & 6.3 & 22.1 & 3.0 & 0.3 & 0.8 & 0.0 & 0.9 & 0.0 & 3.2 \\
\hline 9 & 45.1 & $a 7.4$ & 61.3 & 56.5 & 75.0 & 69.6 & 63.4 & 67.6 & 0.0 \\
\hline $\begin{array}{l}\text { Totel } \\
\text { rune }\end{array}$ & 426 & 321 & 698 & 2798 & 3725 & 92 & 2009 & 386 & 6740 \\
\hline
\end{tabular}


Summerv of Alternation Probability

south cantral

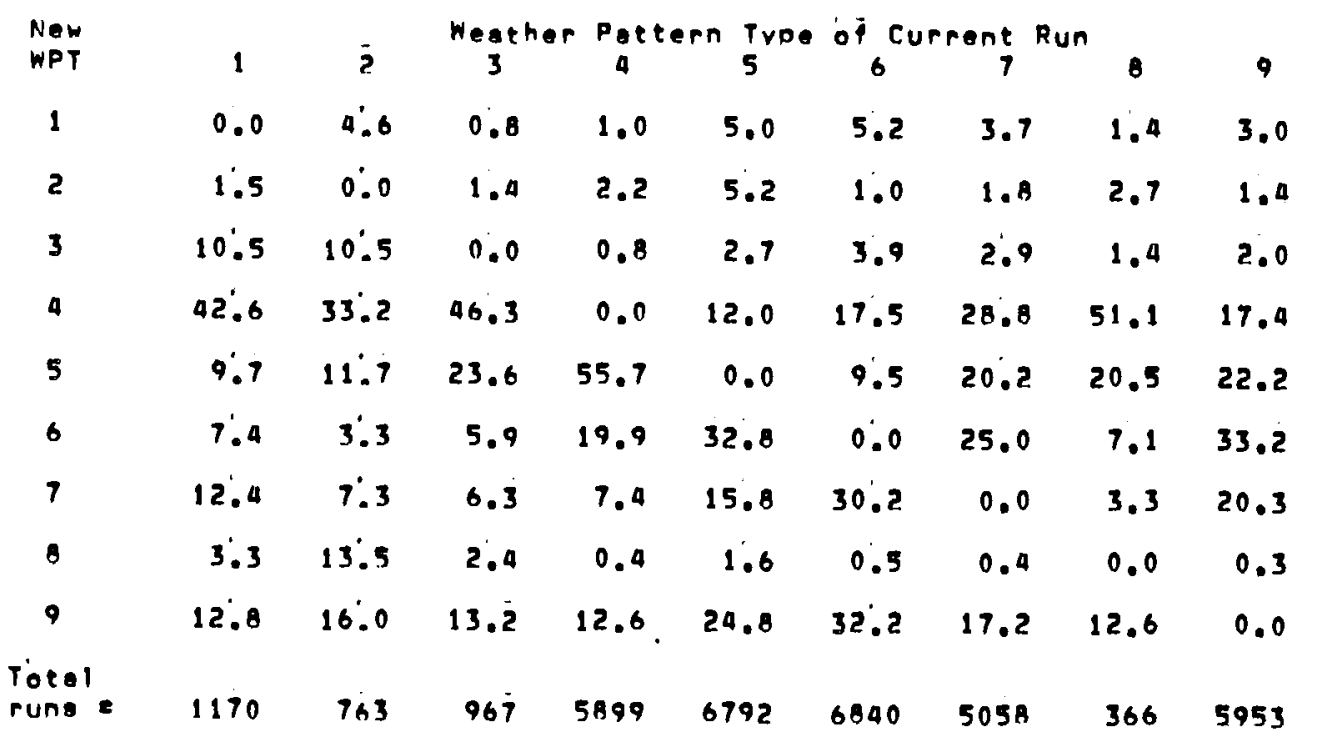

Summerv of Alternation Probability

southeast

\begin{tabular}{|c|c|c|c|c|c|c|c|c|c|}
\hline $\begin{array}{l}\text { New } \\
\text { WPT }\end{array}$ & 1 & 2 & ${ }_{3}^{\text {Weather }}$ & ${ }_{4}^{P} P_{4}$ & ${ }_{5}{ }_{5}$ Tyoe & of $\mathrm{Cu}$ & $\begin{array}{r}\text { rent } \\
7\end{array}$ & Run 8 & 9 \\
\hline 1 & 0.0 & 7.2 & 1.5 & 2.4 & 8.5 & 7.6 & 4.7 & 1.5 & 2.5 \\
\hline 2 & 1.8 & 0.0 & 1.8 & 4.7 & 9.7 & 2.1 & 2.5 & 2.3 & 0.9 \\
\hline 3 & 9.8 & 16.0 & 0.0 & 1.7 & 5.0 & 5.3 & 3.0 & 2.1 & 3.3 \\
\hline 4 & $50^{\circ} .8$ & $30^{\circ} .1$ & 58.3 & 0.0 & 12.4 & 19.5 & 27.8 & 51.7 & 23.1 \\
\hline 5 & 2.9 & 3.8 & 6.5 & 25.7 & 0.0 & 3.4 & 5.4 & 0.5 & 3.0 \\
\hline 6 & 9.7 & 8.1 & 9.5 & 41.4 & 43.2 & 0.0 & 43.7 & 12.2 & 47.0 \\
\hline 7 & $11^{\prime} .2$ & 7.0 & 3.9 & B. 1 & 11.7 & 32.8 & 0.0 & 2.7 & 18.8 \\
\hline 8 & 5.5 & 13.6 & 4.3 & 1.1 & 2.1 & 0.7 & 0.9 & 0.0 & 1.3 \\
\hline 9 & 8.2 & 6.2 & 14.2 & 15.0 & 7.4 & $28^{\circ} .5$ & 12.1 & 18.9 & 0.0 \\
\hline $\begin{array}{l}\text { Total } \\
\text { runa }\end{array}$ & 1354 & 889 & 1201 & 6051 & 2378 & 7867 & 4429 & 518 & 4293 \\
\hline
\end{tabular}





\section{DISTRIBUTION}

No. of

Copies

\section{OFFSITE}

\section{Ancona}

Department of Energy

Wind Energy Technology Division

1000 Independence Avenue

Forrestal Building, Room $5 \mathrm{H} 048$

Washington, DC 20585

C. I. Aspliden

Battelle Memorial Institute Washington Operations Office 2030 M Street, N.W.

Washington, DC 20036

J. Cadogan

Department of Energy

Wind Energy Technology Division

1000 Independence Avenue

Forrestal Building, Room $5 \mathrm{H} 048$

Washington, DC 20585

G. P. Tennyson

Department of Energy

Albuquerque Operations Office

P. 0. Box 5400

Albuquerque, NM 87110

27 DOE Technical Information Center

Dr. K. C. Spengler

American Meteorological Society

45 Beacon Street

Boston, MS 02108

Tom Gray

American Wind Energy Association

1621 Connecticut Avenue, N.W.

Washington, DC 20009

Richard Katzenberg

3100 Highland Place

Washington, DC 20008
No. of

Copies
E. J. Warchol

Bonneville Power Administration P.0. Box 3621

Portland, OR 97208

S. J. Hightower

Bureau of Reclamation

Denver Federal Center

Building 67, Code 254

Denver, CO 80225

Dr. T. McKee

Atmospheric Science Department

Colorado State University

Fort Collins, C0 80521

R. Nolan Clark

Department of Agriculture

S.W. Great Plains

Research Center

Bushland, TX 79012

Dr. Edgar DeMeo

Electric Power Research Institute

3412 Hillview Avenue

Palo Alto, CA 94303

Thomas R. Hiester

Flow Research

21414 68th Avenue, South

Kent, WA 98031

Dr. Wayne Wendland

Illino is State Water Survey

P.0. Box 232

Urbana, IL 61801

James D. West

HQ MAC/DEEE

Scott AFB, IL 62225

Gary G. Worley, Maj. USAF

$\mathrm{HQ}$ AF-ESC/RDVA

Tynda 11 AFB, FL 32403 
Gary L. Johnson

Electrical Engineering Department Kansas State University

Manhattan, KN 66506

Marlatt and Associates

3611 Richmond Drive

Fort Collins, CO 80521

J. Konigsberg

Montana Energy Office

Capital Station

Helena, MT 59601

Phillip French

NASA Scientific and Technical

Information Facility

P.0. Box 8757

Baltimore/Washington International Airport

Baltimore, MD 21240

R. A. Wolf

NASA/Lewis Research Center

Mail Stop 500-201

Cleveland, $\mathrm{OH} 44135$

M. J. Changery

National Oceanic and Atmospheric Administration

National Climatic Center

Federal Building

Asheville, NC 28801

C. F. Chappel 1

Office of Weather Research and Modification

National Oceanic and Atmospheric Administration

Boulder, CO 80303

Dr. N. J. Rosenberg

211 Agricultural Engineering B1dg. University of Nebraska

Lincoln, NE 68583
Prof. J. W. Eng

Department of Soils

Welster Hall

North Dakota State University

Fargo, ND 58102

Dr. A. Eddy

University of $0 \mathrm{klahoma}$

200 Felgar Street, Room 219

Norman, OK 73069

E. W. Hewson/J. E. Wade

Atmospheric Sciences Department

Oregon State University

Corvallis, OR 97331

Terry J. Healy

Rockwel1 International

Rocky Flats Plant

P. 0 . Box 464

Golden, C0 80401

E. Kadlec

Sandia Laboratories

Division 5443

P.0. Box 5800

Albuquerque, NM 87115

5 Dr. P. F. Lester

Department of Meteorology

San Jose State University

San Jose, CA 95192

W. A. Tolbert, Capt. USAF

SERI Site Office/RTLO

1617 Cole Boulevard

Golden, C0 80401

Solar Energy Research Institute

1617 Cole Boulevard

Golden, C0 80401

Mr. Earl L. Kuehnast

Crops Research B1dg., Room 127

University of Minnesota

St. Paul, MN 55101 
No. of

Copies

N. E. Suhs

Tennessee Valley Authority

1360 Commerce Union Bank Building

Chattanooga, TN 37401

Prof. W. Lytle

Agricultural Engineering Bldg. South Dakota State University

Brookings, SD 57006

Prof. J. F. Griffiths

D\&M Building, Room 1027

Texas A\&M University

College Station, TX 77843

Dr. Norman K. Wagner

Department of Civil Engineering

University of Texas

Austin, TX 78712

Dr. Michael Garstang

Department of Environmental

Sciences

University of Virginia

Charlottesville, VA 22903

G. D. Thomann

Wichita State University

P. 0. Box 44

Wichita, KS 67208

Farrell Smith Seiler

Wind Energy Report

Box 14 - 104 S. Village Avenue

Rockville Centre, NY 11571

William Van Dyke

Wind Farms, Ltd.

639 Front Street

San Francisco, CA 94111

Dr. Lyle Horn

Departiment of Meteorology

University of Wisconsin

1225 W. Dayton

Madison, WI 53706
No. of

Copies

Dr. J. Marwitz

Department of Atmospheric Science

University of Wyoming

Laramie, WY 82071

Richard L. Berry

Atmospheric Environment

4905 Dufferin Street

Downsview, Ontario

M3H 5 T4

CANADA

Dr. Neil Cherry

Lincoln College

Canterbury

NEW ZEALAND

ONSITE

1 DOE Richland Operations Office

H. E. Ransom

47 Pacific Northwest Laboratory

W. R. Barchet (5)

J. W. Buck

D. B. Cearlock

J. R. Connell

W. E. Davis (5)

J. C. Doran

C. E. Elderkin

D. L. Elliott

R. L. George

D. L. Hadley

S. K. Heflick

A. H. Miller

D. C. Powell

D. S. Renne

H. L. Wegley

L. L. Wendel1

R. K. Woodruff

Technical Information Library (5)

Publishing Coordination (2)

WCPE Program Office (15) 
